\title{
Instruments and Methods for the Radio Detection of High Energy Cosmic Rays
}

\author{
Zur Erlangung des akademischen Grades eines \\ DOKTORS DER NATURWISSENSCHAFTEN \\ von der Fakultät für Physik des \\ Karlsruher Instituts für Technologie (KIT)
}

genehmigte

DISSERTATION

von

Dipl. Phys. Frank Gerhard Schröder

aus Wiesbaden

Karlsruhe

Dezember 2010

Tag der mündlichen Prüfung: 11. Februar 2011

Referent: Prof. Dr. Johannes Blümer,

Institut für Kernphysik und Institut für Experimentelle Kernphysik

Koreferent: Prof. Dr. Hartmut Gemmeke,

Institut für Prozessdatenverarbeitung und Elektronik 



\section{Abstract}

\section{Instruments and Methods for the Radio Detection of High Energy Cosmic Rays}

Cosmic rays at energies above $\gtrsim 10^{15} \mathrm{eV}$ cannot be measured directly due to the low flux. Instead, the properties of the primary cosmic ray particles (arrival direction, energy, mass) have to be reconstructed from measurements of secondary particles forming an air shower. For this, digital radio antenna arrays, like LOPES at the Karlsruhe Institute of Technology (KIT), are a relatively new instrument. The radio emission mainly originates from the deflection of secondary air shower electrons and positrons in the Earth's magnetic field. The radio technique aims at achieving a similar quality in the reconstruction of air shower parameters as the established Cherenkov or fluorescence light detection methods, which in contrast to the radio technique are limited to dark, moonless nights.

The present studies aim to advance the air shower radio detection in technological aspects and analysis methods. The developments are mainly applied to LOPES, but also provide a useful set of tools, which will soon be applied on the analysis of first AERA measurements. AERA is a next generation digital radio array at the Pierre Auger Observatory in Argentina. Moreover, this thesis reflects the recent progress in the understanding of the radio emission by air showers. The main results of the studies are:

- A new method for time calibration with a reference beacon has been developed. It allows a time resolution of $\sim 1 \mathrm{~ns}$ even with large antenna arrays. This is necessary for digital radio interferometry which improves the signal-to-noise ratio and the reconstruction accuracy of the primary particle properties. This method is essential for the measurement of cosmic rays with LOPES, and is going to be applied at AERA.

- A per-event comparison of lateral distributions measured with LOPES and REAS3 simulations reflects a significantly improved understanding of the radio emission mechanisms. For the first time a Monte Carlo simulation of the radio emission by air showers can in average reproduce measured data. A detailed investigation of systematic effects was performed to accurately reconstruct LOPES lateral distributions. In particular, a method has been developed to appropriately treat the influence of radio noise on measured lateral distributions.

- It is shown that a conical radio wavefront fits LOPES measurements and REAS3 simulations better than a spherical wavefront, which up to now has been assumed for LOPES beamforming analyses. Furthermore, the atmospheric depth of the shower maximum $X_{\max }$ can be reconstructed by determining the opening angle of the conical wavefront. However, due to the small lateral extension of LOPES of about $200 \mathrm{~m}$ and the high radio background at $\mathrm{KIT}$, the measurement uncertainty $\left(\Delta X_{\max } \approx 200 \mathrm{~g} / \mathrm{cm}^{2}\right)$ is too large for a per-event reconstruction of the primary mass. This will improve at AERA, but could not be examined in detail because of a delay in the construction. 


\section{Zusammenfassung}

\section{Instrumente und Methoden zur Radiomessung hochenergetischer kosmischer Strahlung}

Kosmische Strahlung bei Energien $\gtrsim 10^{15} \mathrm{eV}$ kann aufgrund ihres geringen Flusses nicht direkt gemessen werden. Stattdessen müssen die Eigenschaften der Primärteilchen (Ankunftsrichtung, Energie, Masse) aus der Messung der Sekundärteilchen von Luftschauern rekonstruiert werden. Ein vergleichsweise neues Instrument dafür sind digitale Radioantennenfelder, wie LOPES am Karlsruher Institut für Technologie (KIT). Die Radioemission von Luftschauern entsteht hauptsächlich durch die Ablenkung sekundärer Elektronen und Positronen im Erdmagnetfeld. Ziel der Radiomessmethode ist es, eine vergleichbare Rekonstruktionsqualität wie die etablierten Cherenkov- und Fluoreszensmessmethoden zu erreichen, die im Gegensatz zur Radiomessmethode allerdings auf dunkle, mondlose Nächte begrenzt sind.

Diese Arbeit zielt darauf, die Radiomessmethode hinsichtlich Anylsetechniken und technologischer Aspekte weiterzuentwickeln. Die gewonnenen Erkenntnisse werden bereits bei LOPES und bald auch zur Analyse erster AERA-Daten angewendet. AERA ist ein digitales Radiomessfeld der nächsten Generation, das am PierreAuger-Observatorium in Argentinien aufgebaut wird. Darüber hinaus konnte diese Arbeit deutliche Fortschritte beim Verständnis der Radioemission erreichen. Die wesentlichen Ergebnisse sind:

- Eine neue Methode zur Zeitkalibration mit einen Rerefenzsender (Beacon) wurde entwickelt, die auch bei großen Radiomessfeldern eine Zeitauflösung von $\sim 1 \mathrm{~ns}$ ermöglicht. Dies ist notwendig, um digitale Interferometrie zu betreiben und so das Signal-zu-Rausch-Verhältnis und damit die Rekonstruktionsgenauigkeit der Primärteilcheneigenschaften zu erhöhen. Diese Methode ist wesentlich für Messungen mit LOPES und wird auch bei AERA verwendet.

- Ein Vergleich der Lateralverteilungen einzelner LOPES-Ereignisse mit REAS3Radiosimulationen spiegelt ein deutlich verbessertes Verständnis der Radioemissionsmechanismen wider. Zum ersten Mal kann eine Monte-Carlo-Simulation gemessene Radiodaten reproduzieren. Bei der Rekonstruktion der LOPESLateralverteilungen sind verschiedener Systematiken von Belang. Insbesondere wurde eine Methode entwickelt, die den Einfluss von Radiorauschen auf gemessene Lateralverteilungen angemessen berücksichtigt.

- Es wird gezeigt, dass eine konische Wellenfront besser zu LOPES-Messungen und REAS3-Simulationen passt als eine sphärische Wellenfront, wie sie bisher in LOPES-Beamforming-Analysen angenommen wurde. Zudem kann die atmosphärische Tiefe des Schauermaximums $X_{\max }$ aus Messungen der Radiowellenfront bestimmt werden. Wegen der kleinen Ausdehnung von LOPES von etwa $200 \mathrm{~m}$ und des hohen Radiountergrunds am KIT, ist der Messfehler mit $\Delta X_{\max } \approx 200 \mathrm{~g} / \mathrm{cm}^{2}$ allerdings zu groß für eine Massenbestimmung einzelner Primärteilchen. Bei AERA ist eine höhere Genauigkeit zu erwarten, was aufgrund von Verzögerungen beim Aufbau von AERA allerdings nicht näher untersucht werden konnte. 


\section{Contents}

Abstract II

$\begin{array}{ll}\text { 1. Introduction } & 1\end{array}$

2. Cosmic Rays 3

2.1. Origin of cosmic rays . . . . . . . . . . . . . . 3

2.2. Cosmic ray air showers . . . . . . . . . . . . . . . 7

2.3. Classical measurement techniques for air showers . . . . . . . . 8

2.4. Radio emission from air showers . . . . . . . . . . . . . . 99

2.4.1. Features of air shower induced radio pulses . . . . . . . . 10

2.4.2. Emission mechanisms . . . . . . . . . . . . . . 11

3. Radio Experiments for Air Shower Detection $\mathbf{1 5}$

3.1. Overview of modern radio experiments . . . . . . . . . . 15

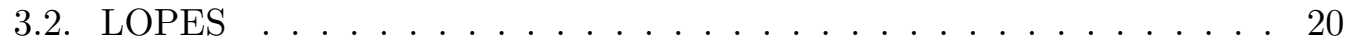

3.2.1. Setup of LOPES . . . . . . . . . . . . . . . 20

3.2.2. LOPES analysis pipeline . . . . . . . . . . . . . . 22

3.3. Radio detection at the Pierre Auger Observatory . . . . . . . . . . . 26

3.3.1. Radio prototype setups . . . . . . . . . . . . . 27

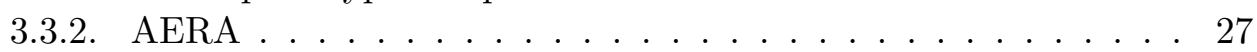

3.3.3. Offline analysis software . . . . . . . . . . . . . 29

4. Time Calibration of LOPES $\quad 31$

4.1. Amplitude calibration . . . . . . . . . . . . . . . . 31

4.2. Need for a precise time calibration . . . . . . . . . . . . . 33

4.3. Measurement of antenna positions . . . . . . . . . . . . 36

4.4. Measurement of the relative delays . . . . . . . . . . . . . . 38

4.5. Pulse distortion by dispersion . . . . . . . . . . . . . . . . 41

4.6. Monitoring of the timing with a beacon . . . . . . . . . . 45

4.7. Conclusions on the time calibration of LOPES $\ldots \ldots$. . . . . . 49

5. A Reference Beacon for the Auger Engineering Radio Array 51

5.1. Proof of principle . . . . . . . . . . . . . . 52

5.1.1. Test measurements at the AERA site . . . . . . . . . 53

5.1.2. Test measurements at the BLS . . . . . . . . . . . 54

5.1.3. Conclusions for AERA . . . . . . . . . . . . 58

5.2. AERA Beacon system . . . . . . . . . . . . . . 58

5.2.1. Design and installation . . . . . . . . . . . . 58

5.2.2. Performance of the beacon . . . . . . . . . . . 64

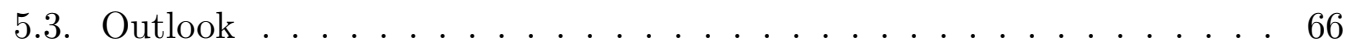


6. Treatment of Noise $\quad \mathbf{6 7}$

6.1. Noise and RFI . . . . . . . . . . . . . . . . 6 67

6.2. Measurement of signal and noise with LOPES . . . . . . . . . . . . 70

6.3. Influence of noise on pulse amplitudes . . . . . . . . . . . 76

6.4. Influence of noise on lateral distributions . . . . . . . . . . . . . . 82

6.5. Influence of noise on pulse arrival time measurements . . . . . . . . . . 84

$\begin{array}{lr}\text { 7. Lateral Distribution } & \mathbf{8 7}\end{array}$

7.1. Analysis procedure . . . . . . . . . . . . . . . . . 87

7.1.1. Determination of the amplitude . . . . . . . . . . . 88

7.1.2. Determination of the lateral distance . . . . . . . . . . . . . 89

7.1.3. Exponential fit . . . . . . . . . . . . . . . . 90

7.2. Event selection . . . . . . . . . . . . . . . . . . 92

7.3. Systematic effects . . . . . . . . . . . . . . . . . . 95

7.3.1. Antenna height and shower inclination . . . . . . . . . 96

7.3.2. Pulse distortion . . . . . . . . . . . . . . . . . . 97

7.3.3. Frequency band . . . . . . . . . . . . . . . . . 98

7.3.4. Up-sampling . . . . . . . . . . . . . . . . . . . . . 98

7.4. Comparison of LOPES data and REAS3 simulations . . . . . . . . . 100

7.4.1. Test of REAS3 simulations against LOPES data . . . . . . . 100

7.4.2. Mass sensitivity . . . . . . . . . . . . . . . . . . . 104

7.5. Conclusion . . . . . . . . . . . . . . . . 106

$\begin{array}{ll}\text { 8. Pulse Arrival Time Distributions } & 107\end{array}$

8.1. Selection: LOPES events and REAS3 simulations . . . . . . . . . . . 109

8.2. Analysis procedure . . . . . . . . . . . . . . . . . 109

8.2.1. Pulse arrival time measurement . . . . . . . . . . . . . 111

8.2.2. Fit of the arrival time distribution . . . . . . . . . . . 112

8.2.3. Comparison of curvature reconstruction methods . . . . . . . . 115

8.3. Investigation of the wavefront shape . . . . . . . . . . . . . . 117

8.3.1. Spherical wavefront . . . . . . . . . . . . . 117

8.3.2. Conical wavefront . . . . . . . . . . . . . . 120

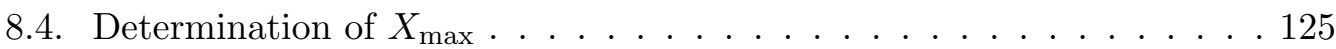

8.4.1. $X_{\max }$ reconstruction with curvature method . . . . . . . 125

8.4.2. $X_{\max }$ reconstruction with cone method . . . . . . . . 126

8.4.3. Mass sensitivity . . . . . . . . . . . . . . . . 131

8.5. Conclusion . . . . . . . . . . . . . . . . . . 132

$\begin{array}{ll}\text { 9. Conclusion } & 135\end{array}$

$\begin{array}{lr}\text { Appendix } & 137\end{array}$

$\begin{array}{ll}\text { A. Radio Measurements at Tunka } & 137\end{array}$

B. Up-Sampling and Envelope 143

$\begin{array}{ll}\text { C. LOPES Antenna Positions } & 145\end{array}$ 
$\begin{array}{lr}\text { D. Example Events } & 147\end{array}$

$\begin{array}{ll}\text { Bibliography } & 161\end{array}$

$\begin{array}{ll}\text { List of Figures } & 173\end{array}$

$\begin{array}{ll}\text { List of Tables } & 177\end{array}$

$\begin{array}{lr}\text { List of Abbreviations } & 179\end{array}$ 



\section{Introduction}

Almost a hundred years after their discovery, cosmic rays still have preserved some mysteries and fascinate an international community of scientists. By now, the acceleration of atomic nuclei by super nova shock fronts is a well established paradigm which can, at least partly, explain the origin of the galactic cosmic rays with energies $\lesssim 10^{15} \mathrm{eV}$. Yet, the nature and origin of particles at highest energies up to a few $10^{20} \mathrm{eV}$ is not solved. Neither the end of the energy spectrum is known, nor the energy of the transition from galactic to extragalactic cosmic rays. One reason for this is that the flux of cosmic rays at the highest energies is extremely low. Thus, they can only be detected indirectly by measuring air showers of secondary particles. Consequently, answering those questions requires large air shower observatories, and methods to reconstruct the properties of the primary cosmic ray particles from the air shower observables, namely its energy, arrival direction, and type, respectively mass. The established methods rely on detection of secondary particles at ground, fluorescence and Cherenkov light emitted by the air shower. The first one allows the reconstruction of the primary particle properties only within large uncertainties due to statistical fluctuations and uncertainties of the models for shower generation and hadronic interactions at the highest energies. The latter ones suffer from limited operation time restricted to dark nights (see chapter 2).

Radio measurements of air showers have the potential to combine the advantages of these established techniques (good reconstruction capabilities and high duty cycle). Historic experiments already showed that air shower induced radio pulses can in principle be used to reconstruct the primary energy and arrival direction, but neither the measurement precision nor the understanding of the radio emission mechanism was sufficient to compete with the other detection techniques.

This situation started to change when LOPES, a digital antenna array co-located with the KASCADE-Grande experiment at KIT, proved that air showers can be measured with radio interferometry (see chapter 3 ). Nevertheless, to make the radio detection technique a feasible tool for cosmic ray physics, the reconstruction accuracies of arrival direction, energy and mass must be ameliorated, and the technical applicability to large scale experiments must be demonstrated. Both of these are goals of the Auger Engineering Radio Array (AERA) at the Pierre Auger Observatory in Argentina.

To achieve these goals, research on the following topics is required. First, the precision of the measurement of the amplitude and arrival time of the radio emission has to be enhanced. The arrival direction is essentially reconstructed by measuring arrival times, and the energy by measuring amplitudes. The primary mass can be probed either by the shape of the wavefront, i.e., by pulse arrival times, or by the slope of the lateral distribution of pulse amplitudes. Second, the theoretical understanding of the radio emission has to be improved, which can be done by comparing model predictions with measurements. Third, all techniques, be it data processing, or 
calibration methods must be scalable to large antenna array, for probing cosmic rays at highest energies.

This thesis made progress in all the three research topics. Like LOPES has already shown, the reconstruction of arrival direction, energy and mass can be done with interferometric beamforming. This technique improves the signal-to-noise ratio compared to analyses based on pulse arrival times and amplitudes at individual antennas. The precision of interferometric beamforming depends strongly on the relative timing accuracy and precision between different antennas. Thus, a new method for the time calibration of LOPES is introduced in chapter 4 . It allows to achieve the necessary timing resolution of $\lesssim 1$ ns per event by continuously measuring the phase of a reference signal emitted by a radio beacon.

This beacon technique has been made applicable to large scale experiments of autonomous stations with independent clocks (e.g., GPS). Such a beacon was deployed and tested at AERA (see chapter 5). Without the beacon the relative timing of AERA would be insufficient for digital interferometry, and AERA could only rely on the analysis of lateral distributions and pulse arrival time distributions of the radio signal.

As shown in chapter 6, especially for antennas with signals close to the noise level (e.g., at large lateral distances), noise can be the dominant source of error for time and amplitude measurements. Moreover, noise systematically flattens the lateral distribution. Hence, accounting for noise is an important issue for the reconstruction of shower parameters based on amplitude and time measurements in individual antennas.

One motivation to look not only at the interferometric combination of all antennas, but also at individual antennas, is that the lateral distribution is an excellent tool to compare theoretical models for radio emission with measured data. Because of the better comprehension of noise and other systematic effects, the precision of LOPES measurements has become sufficient to test recently improved models for radio emission, like REAS3 (see chapter 7). LOPES measurements and REAS3 simulations generally match each other, which demonstrates that our understanding of the radio emission has greatly improved. Furthermore, the comparison of measurement and simulation confirms that the lateral distribution provides a method to reconstruct the primary mass.

Beside that, the mass sensitive shower maximum $X_{\max }$ can also be estimated by reconstructing the radio wavefront with pulse arrival time measurements (see chapter 8). The radio wavefront of LOPES measurements as well as REAS3 simulations can better be described with a cone than with a sphere. This is a new result, since up to now a spherical wavefront has been assumed in all LOPES beamforming analyses. $X_{\max }$ can be estimated from the opening angle of the conical wavefront. Although this method in principle works and yields $X_{\max }$ values in the expected order of magnitude, it became clear that uncertainties at LOPES are too large for a per-event reconstruction of $X_{\max }$. Limiting factors at LOPES are the high level of ambient noise, and the small extension of the antenna array $(\sim 200 \mathrm{~m})$. Hence, the approach is expected to be more successful at AERA, which by the end of 2010 has started to measure the radio emission of air showers in a less noisy environment and on larger scale. This thesis provides several techniques which will soon be applied on the analyses of first AERA data. 


\section{Cosmic Rays}

A variety of different types of radiation reaches the Earth's atmosphere, covering many orders of magnitude in flux and energy: from very low energies below $10^{-3} \mathrm{eV}$, like the cosmic microwave background radiation [1], to the highest energies of charged cosmic ray particles with up to a few $10^{20} \mathrm{eV}$ [2] (see figure 2.1). The extraterrestrial origin of cosmic rays was discovered by Victor Hess in 1912, when he measured an increase of ionizing radiation with height during balloon ascents [3]. Usually, the term 'cosmic rays' only refers to those particles coming from outside our solar system, i.e., particles with energies above $\sim 10^{9} \mathrm{eV}$. This cosmic radiation consists mainly of protons, but heavier atomic nuclei, electrons, positrons, anti-protons, gamma rays and neutrinos are also present. Sometimes, gamma rays and neutrinos do not count as cosmic rays, because the cosmic accelerators are believed to accelerate only charged particles, and thus any uncharged particle ought to be a secondary product of a charged primary cosmic ray particle. Even today, it is not totally clear what the sources of cosmic rays are and which mechanism is responsible for the acceleration of cosmic rays at the highest energies [4].

Since the flux of cosmic rays decreases rapidly with energy, different techniques are required to detect lower energy particles $\left(E \lesssim 10^{14} \mathrm{eV}\right)$ and higher energy particles $\left(E \gtrsim 10^{13} \mathrm{eV}\right)$. Lower energy cosmic rays can be measured directly with particle detectors on balloons or space craft. Higher energy cosmic rays are so rare that a detection with sufficient statistics needs larger detection areas than direct measurements can provide. Thus, indirect measurement methods are used, based on the detection of extensive air showers induced by cosmic rays. These air showers consist of secondary particles generated in the atmosphere when a primary cosmic ray particle interacts with air molecules. Air showers can be detected either by particle detectors on ground or by measuring electromagnetic radiation induced by the air shower particles in the atmosphere. The difficulty lies in the reconstruction of the primary cosmic ray particle properties, i.e., its arrival direction, energy and mass.

This chapter gives a short introduction to the current knowledge about the origin of cosmic rays, the development of air showers and the techniques to measure them. Special emphasis is given to the emission and detection of air shower induced radio pulses - a relatively new and promising approach for cosmic ray detection at ultra high energies.

\subsection{Origin of cosmic rays}

The almost uniform power law of the cosmic ray energy spectrum indicates that the acceleration of primary particles is performed in a similar way at all energies, but not necessarily by the same type of source. A favored model is the relativistic shock front acceleration (first order Fermi acceleration) $[5,6]$ : When a relativistic particle is reflected by a shock front, it gains energy as the shock front is moving towards the 


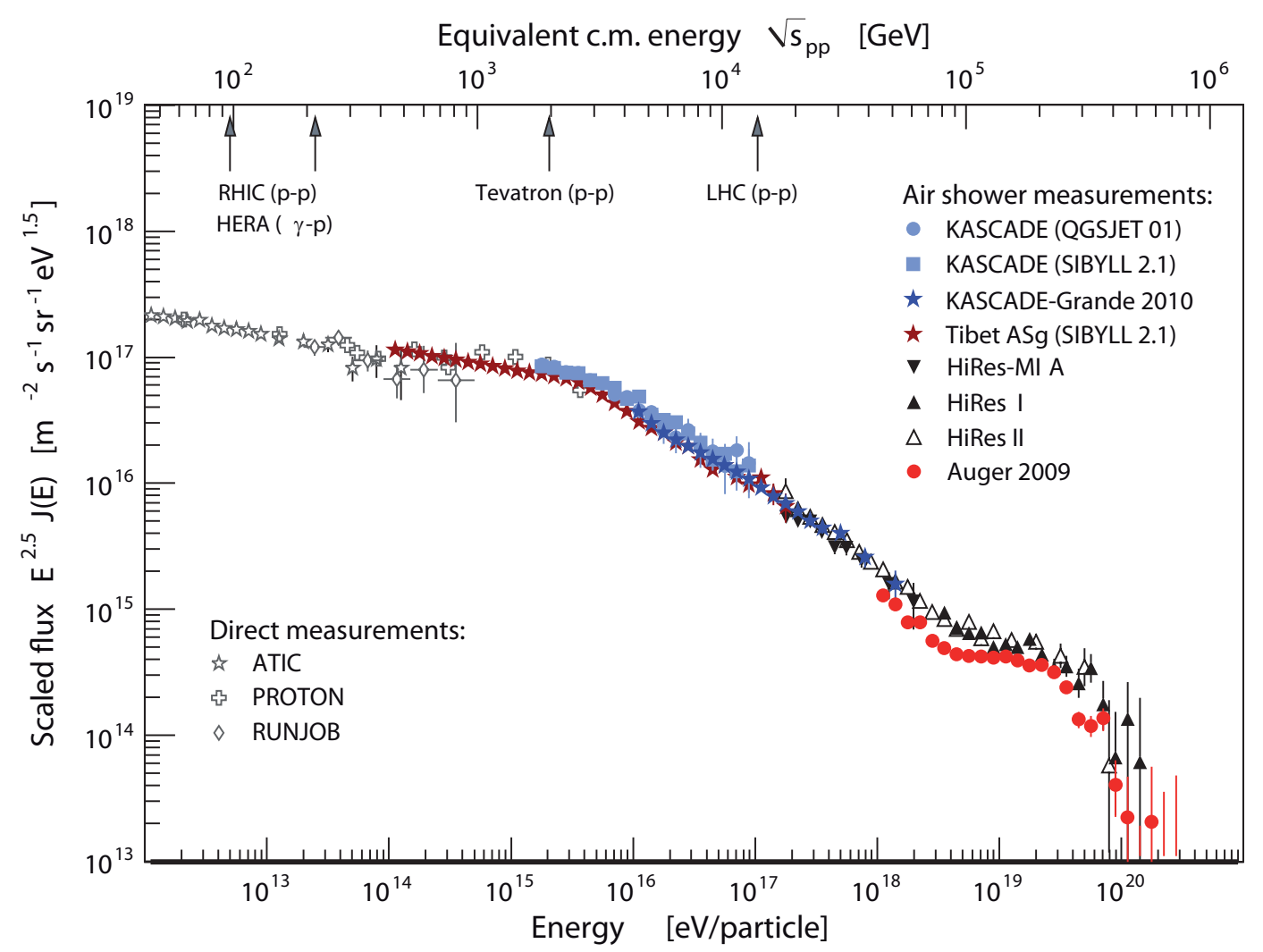

Figure 2.1.: Cosmic ray energy spectrum from exemplary chosen direct balloon and satellite experiments, and indirect air shower based measurements [4].

particle (like a tennis ball is hit and reflected by a racket). Although existing shock fronts in space do not reflect particles instantaneously, but gradually by magnetic fields, the basic principle of acceleration holds true. Due to a certain probability for the particles to escape form the acceleration zone after each reflection at the shock front, a power law energy spectrum is generated. The power law index is predicted to be around -2 , and thus slightly higher than the spectral index measured at Earth $\left(-2.7\right.$ for $\left.E \lesssim 10^{15} \mathrm{eV}\right)$. But cosmic ray propagation models for the magnetic fields in the Milky Way and in intergalactic space can explain the observed discrepancy [7].

The question remains where to find the accelerating shock fronts. Galactic cosmic rays at lower energies $\left(\lesssim 10^{14} \mathrm{eV}\right)$ originate at least partly from shock fronts generated by super nova explosions, so called super nova remnants. The evidence for this is threefold. First, the observation of gamma rays from super nova remnants with energies up to $\sim 10^{14} \mathrm{eV}[8]$ proves that they can accelerate primary particles at least up to that energy, and probably to even higher energies because gamma rays are supposed to be emitted by accelerated charged particles or secondary pions. However, these charged primary particles might be predominantly electrons, and not or only partially nuclei. Second, the composition of galactic cosmic rays matches the expectations from models, which assume the acceleration taking place in OB associations [9]. These are regions of the Milky Way with a high rate of super novae 


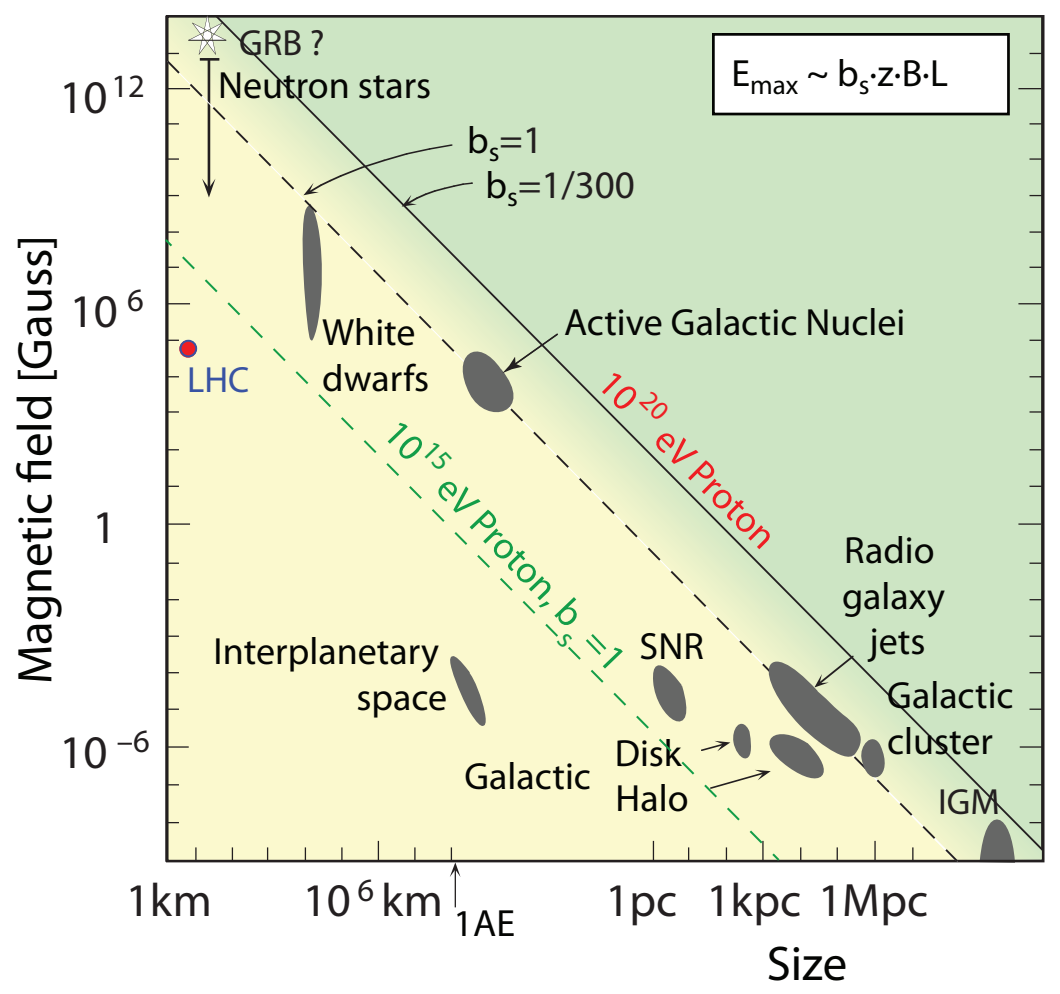

Figure 2.2.: Hillas plot of candidate sources for acceleration of ultra high energy cosmic rays (after [10]).

explosions. Third, the energy output of super novae in our galaxy is sufficient to explain the observed flux of cosmic rays.

At higher energies, the situation is less clear. At the knee $\left(\sim 10^{15.7} \mathrm{eV}\right)$, the slope of the cosmic ray energy spectrum steepens, since the flux of light nuclei decreases and the primary composition becomes heavier [11]. Hence, the knee could indicate the maximum energy reachable by super nova shock front acceleration. This picture gets additional support because at higher energies $\left(\sim 10^{17} \mathrm{eV}\right)$, another kink in the spectrum is observed. It is at about 26 times the energy of the knee and could indicate the maximum energy for iron nuclei $(Z=26)$ reachable by super nova shock front acceleration [12]. A second explanation for this features would be the leakage of cosmic rays from our Milky Way, since the galactic magnetic fields might not be strong enough to bind them anymore at these energies. In both cases, it is likely that another type of source is responsible for cosmic rays at energies beyond $\sim 10^{17} \mathrm{eV}$.

In principle, a list of source candidates can be obtained by looking at the magnetic field strength $B$ at a possible source and its extension $L$. The product $B L$ indicates how long cosmic ray particle can remain in the accelerating source region. Hence, the size and magnetic field strength of a source candidate leads to a theoretical maximum energy which can be reached by any electromagnetic acceleration process. Such a candidate selection was first be done by Hillas [10], and yields several galactic and extra-galactic source candidates like neutron stars, gamma ray bursts, and active galactic nuclei (see figure 2.2). The transition energy from galactic to extra-galactic 


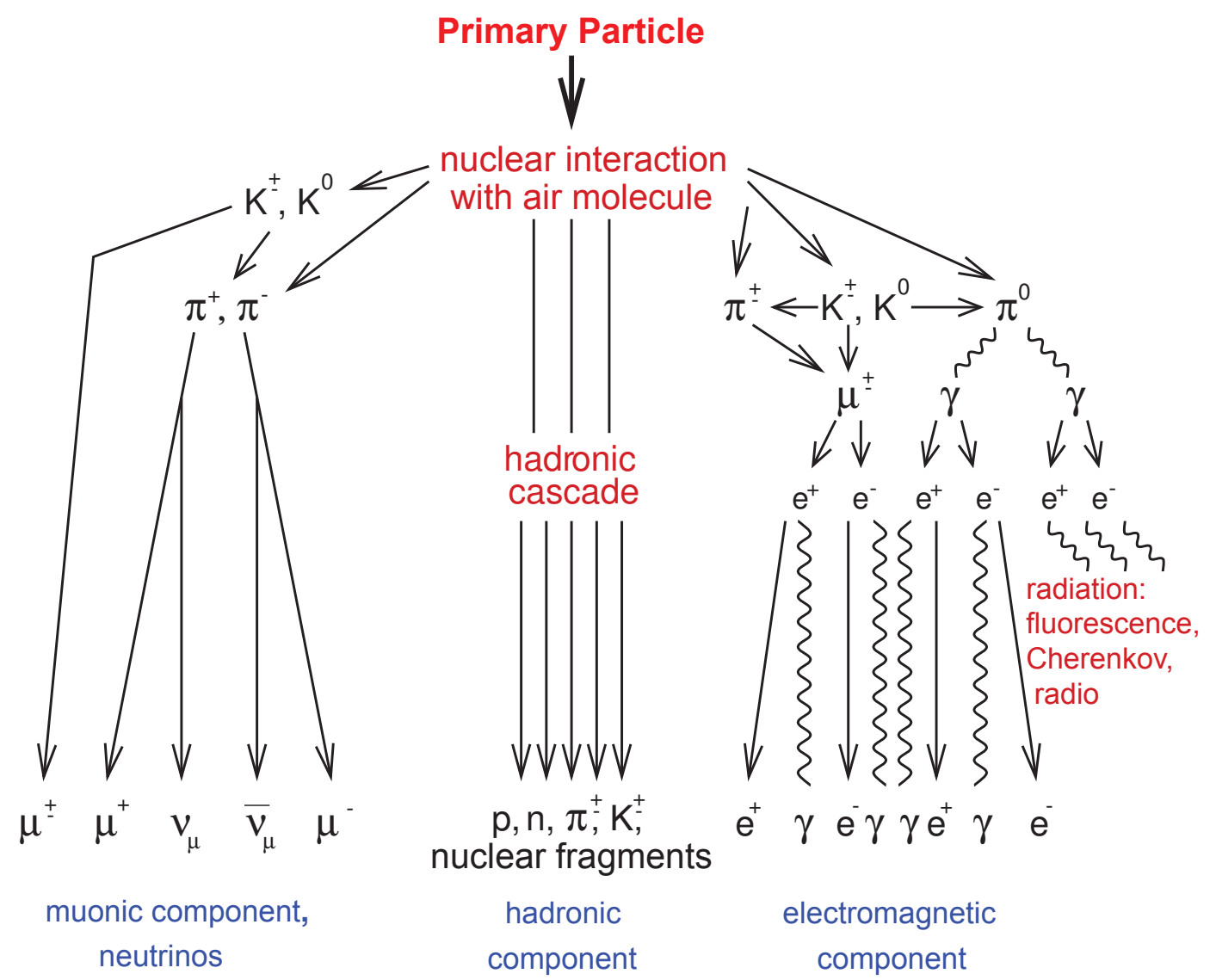

Figure 2.3.: Scheme of the development of an extensive air shower initiated by a cosmic ray nucleus (after [20]).

cosmic rays is not known, yet. It might be at the ankle, a flattening in the spectrum at $\sim 10^{18.3} \mathrm{eV}$.

From observations, it is only known that at least a part of the ultra high energy cosmic rays $\left(E \gtrsim 10^{19.6} \mathrm{eV}\right)$ is coming from outside our galaxy, since the arrival direction of those cosmic rays are correlated with the distribution of nearby $(\lesssim$ $100 \mathrm{Mpc}$ ) galaxies $[13,14]$. This is consistent with expectations, as protons with higher energies should not be able to travel larger distances. This is because of the GZK cutoff $[15,16]$ : higher energy protons would interact with photons of the cosmic microwave background and loose energy. For heavier nuclei there exist similar, but slightly different cutoffs, e.g., due to photo dissociation [17].

Solving the open questions of ultra high energy cosmic ray physics, especially the question of their origin, requires accurate and precise measurements of the flux, energy, arrival direction and particle type (mass) of primary cosmic rays. Because the flux at the highest energies is extremely low, this can only be done by experiments with a large exposure, i.e., by huge air shower observatories like the Pierre Auger Observatory [18], or by future space craft observatories, like JEM-EUSO [19]. Moreover, systematic uncertainties demand a combination of several detection techniques to increase the measurement accuracy. 


\subsection{Cosmic ray air showers}

When ultra high energy cosmic rays hit the Earth's atmosphere they create particle air showers [21], similar to the particle showers known from calorimeters in high energy physics (see figure 2.3). Understanding the properties of air showers in detail requires simulations which can, for example, be accomplished with AIRES [22] or CORSIKA [23]. A primary particle of a certain energy, mass and incoming direction is assumed. Its interaction with the atmosphere as well as the interactions of all secondary particles are modeled with a Monte Carlo simulation. Since the relevant energy scale exceeds the range of accelerator experiments, measured cross-sections are extrapolated. Thus, air shower models and simulations can only approximately describe nature, with little, but significant deviations (see e.g., [24]). The basic features of air showers can already be understood without simulations, from simple phenomenological considerations. Such a phenomenological air shower model can be found in [25], for example. A summary is presented here.

When the primary cosmic ray particle, e.g., a nucleus, scatters inelastically with an air nucleus, it generates secondary particles of different types, which interact themselves with other air nuclei. These interactions lead to electromagnetic or hadronic cascades, depending on the particle type. High energy gammas and electrons (or positrons) induce electromagnetic cascades. In a simple model, each photon creates an electron-positron-pair after a splitting length $d$, and each electron above a critical energy of about $85 \mathrm{MeV}$ looses half its energy per splitting length $d$ by radiating a photon $\left(d=\lambda_{r} \ln 2\right.$ with the radiation length $\lambda_{r} \approx 37 \mathrm{~g} / \mathrm{cm}^{2}$ in air). Electrons below the critical energy will be absorbed by the atmosphere.

Hadronic cascades are initiated by nuclei and hadrons. They interact with air nuclei and produce mainly mesons such as charged and uncharged pions, and kaons. In a simple model kaons are neglected, and in each interaction, one third of the energy is alloted to neutral pions, which decay immediately into photons and feed the electromagnetic cascade. The other two thirds of the energy are alloted to charged pions which will interact again after an interaction length of about $120 \mathrm{~g} / \mathrm{cm}^{2}$ (in air), until the energy of the charged pions drops below $\sim 20 \mathrm{GeV}$. Then, the pions will decay into muons, before another interaction can take place. Because of their relativistic speed, most of the muons will reach the ground before decaying. The number and energy of hadrons reaching ground level is negligible for most practical purposes, except for high altitudes or close to the shower axis. Thus, most experiments concentrate on measuring muons and electromagnetic particles.

The primary energy can be estimated from the electron and muon numbers $N_{\mathrm{e}}$ and $N_{\mu}$ at observation level: $E_{0} \approx a \cdot\left(N_{\mathrm{e}}+b N_{\mu}\right)$ at sea level, with $a$ and $b$ depending on the detection thresholds $(a=0.85 \mathrm{GeV}$ and $b=25$ in the simplified model without detection thresholds [25]). At primary energies of $10^{17} \mathrm{eV}$, about $90 \%$ of the shower energy is in the electro magnetic cascade. Consequently, the primary energy can - within some uncertainties - be obtained by measuring alone the electromagnetic shower component.

The shower maximum $X_{\max }\left[\mathrm{g} / \mathrm{cm}^{2}\right]$ is the atmospheric depth, where the number of particles reaches its maximum. $X_{\max }$ is sensitive to the type of the primary particle because photons (and neutrinos) are assumed to interact on average more deeply in the atmosphere than nuclei. Moreover, $X_{\max }$ is even sensitive to the mass of the primary nuclei. In a simplified model, at high energies each nucleon is supposed 
to interact separately with atmospheric nuclei. Hence, at the same primary energy, heavier nuclei will on average interact earlier in the atmosphere, and give rise to fewer generations of secondary particles. Consequently, the muon number is increased with respect to proton showers by $N_{\mu}^{A}=N_{\mu}^{\mathrm{p}} \cdot A^{0.15}$, and $X_{\max }$ is lower. Typical $X_{\max }$ values at primary energies of $10^{17} \mathrm{eV}$ are $580 \mathrm{~g} / \mathrm{cm}^{2}$ for iron showers and $680 \mathrm{~g} / \mathrm{cm}^{2}$ for proton showers.

\subsection{Classical measurement techniques for air showers}

Classical measurement techniques for air showers rely either on particle detection on ground or indirect measurements of the energy content of the electromagnetic cascade, e.g., by observing fluorescence or Cherenkov light produced by the electrons when traveling through the atmosphere. As Cherenkov light measurements are important mainly for gamma ray observation at energies of $\sim \mathrm{TeV}$, they are not discussed here. The detection of secondary particles on ground and fluorescence light are well established and successful techniques for cosmic ray measurements at ultra high energies. Their main advantages and disadvantages are presented in the following paragraphs (see also table 2.1).

Detecting the secondary particles on ground requires large and expensive arrays of particle detectors, like scintillators or water Cherenkov counters. They have the advantage of a close to $100 \%$ duty cycle and a relatively simple determination of the exposure, which is mandatory for evaluation of the cosmic ray flux at a certain energy. Furthermore, the arrival direction of the primary particle can easily be obtained from the relative particle arrival times at different detector stations. However, ground arrays have the disadvantage that they measure only one stage of the shower development, namely when the shower intersects the ground plane. The primary energy can be estimated from the total number of detected secondary particles and the primary mass from the ratio of electrons and muons. However, fluctuations in the shower development and extrapolations of the interaction models beyond the range probed by accelerator experiments lead to relatively large uncertainties.

Fluorescence telescopes allow the detection of air showers in a large area by observing fluorescence light of nitrogen atoms excited by the electromagnetic cascade. The intensity of the fluorescence light is proportional to the primary energy within comparably small uncertainties. Furthermore, fluorescence measurements allow a precise determination of $X_{\max }$ (with $\Delta X_{\max } \sim 20 \mathrm{~g} / \mathrm{cm}^{2}$ ). However, they have the big disadvantage that they are limited to dark, moonless nights resulting in a limited duty cycle of about $10 \%$. In addition, it is difficult to estimate the exposure because it is uncertain, up to which maximum distance an air shower at a specific energy can be seen.

Most of the disadvantages of both detection techniques can be overcome by combining them in a hybrid experiment. Consequently, two cosmic ray experiments of the latest generation, namely the Pierre-Auger-Observatory [18] and the Telescope Array [26], consist of fluorescence telescopes taking data simultaneously with a particle detector array. Still, even in these hybrid experiments, high quality data are limited by the low duty cycle of $\sim 10 \%$ of fluorescence measurements. Thus, complementary detection methods with a higher duty cycle are explored, and one of the most promising is the detection of radio pulses emitted by air showers. 
Table 2.1.: Performance comparison of different techniques for air shower measurement $^{a}$ : surface particle detectors (SD), fluorescence light detectors (FD) and digital radio arrays $(\mathrm{RD})$

\begin{tabular}{lccc} 
& $\mathrm{SD}$ & $\mathrm{FD}$ & $\mathrm{RD}$ \\
\hline angular resolution & + & $-/ \mathrm{o}^{b}$ & + \\
energy reconstruction accuracy & $\mathrm{O}^{c}$ & + & $\mathrm{o} /+^{d}$ \\
sensitivity to primary mass & $-^{c}$ & + & $-/+^{d}$ \\
determination of exposure & + & - & - \\
duty cycle & $\sim 100 \%$ & $\sim 10 \%$ & $\sim 95 \%$ \\
energy threshold & $\sim 10^{14} \mathrm{eV}$ & $\sim 10^{17} \mathrm{eV}$ & $\sim 10^{17} \mathrm{eV}$ \\
\hline Legend: + good, o average, - poor & & & \\
\hline
\end{tabular}

${ }^{a}$ This table reflects the author's personal view of how the different detection techniques compare with each other. The performances of surface detector arrays and fluorescence telescopes are intrinsically better when used in hybrid combination.

${ }^{b}$ Mono / stereo mode; even better in hybrid combination.

${ }^{c}$ Due to systematic uncertainties of air shower models.

${ }^{d}$ demonstrated, current status / expectation after further research, including detector development and better understanding of the radio emission mechanism

${ }^{e}$ principal limit, e.g., due to light and weather conditions, not including down-time and maintenance

\subsection{Radio emission from air showers}

The measurement of the radio emission of the electrons and positrons in an air shower is an alternative, and relatively new technique for cosmic ray detection. The principal features of the air shower induced radio emission have already been predicted $[27,28]$, and discovered about 50 years ago [29]. However radio detection of air showers became feasible only after the development of digital radio antenna arrays [30]. Since then, interest in this method has increased, and numerous researches and developments are performed to explore the prospects of the radio technique for cosmic ray air shower detection.

The amplitude of the radio pulse is related to the number of particles and the energy of the electromagnetic cascade, which provides a calorimetric measurement of the primary energy, similar to the fluorescence method. By its sensitivity to the longitudinal shower development, measuring the radio emission should thus also provide a method for $X_{\max }$ determination, and hence, the type and mass of the primary particle (cf., chapter 8 ). In addition, radio antenna arrays share two principal advantages of particle detector arrays: the arrival direction can be determined relatively simply by measurements of the pulse arrival times, and the duty cycle has no principle limitation. In fact, the duty cycle can reach almost $100 \%$, as recent experience shows that only periods with nearby thunderstorms and high atmospheric electric fields have to be excluded [31,32], resulting in a realistic duty cycle of a few percent below that of particle detector arrays.

Nonetheless, there are some drawbacks. First, the detection threshold and the efficiency at a certain energy depend on the shower arrival direction, which makes a determination of the exposure and cosmic ray flux difficult, if radio antennas are not used in combination with a particle detector array. Second, it has still to be verified 


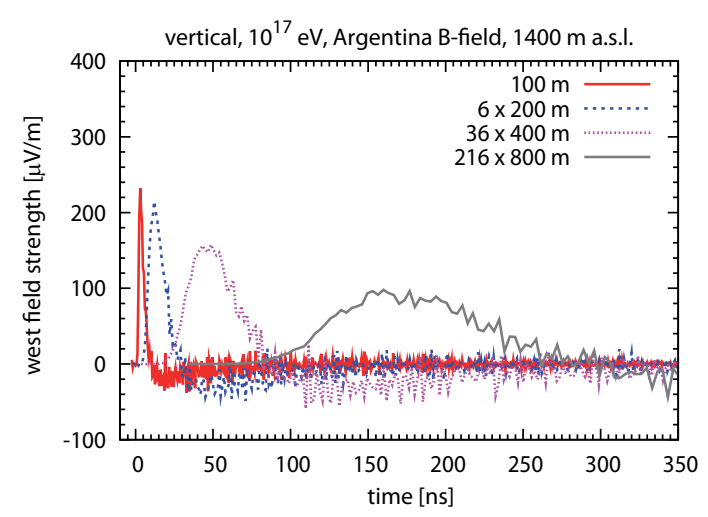

(a) unfiltered radio pulses (REAS3)

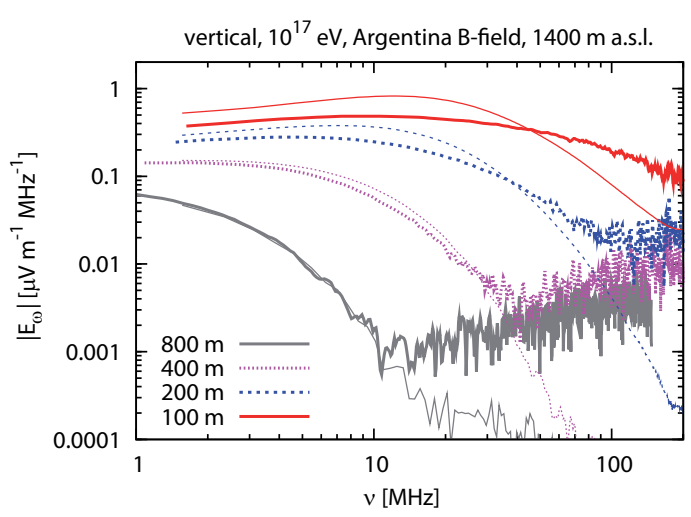

(b) frequency spectra (REAS3 + MGMR)

Figure 2.4.: Simulated radio emission for different lateral distances to the air shower axis [33]. Left: REAS3 simulation of the east-west polarization component. The bipolar structure of the radio pulses is only marginally visible due to numerical noise. Right: REAS3 (thick lines) and MGMR (thin lines) simulations of the spectrum. The rising field strength towards higher frequencies is an artifact of the numerical noise.

that the precision of primary energy and mass reconstruction can be as good as those of fluorescence measurements.

To overcome the current drawbacks and exploit the full potential of the radio detection technique, a detailed understanding of the emission process is mandatory. This is done by modeling the radio emission and comparing predictions of simulations with measurements. Furthermore, the performance of radio arrays can be crosschecked with coincident measurements in hybrid experiments. Whereas the current experimental situation is reviewed in the next chapter, the basic features of the radio emission and the status of our understanding of the emission process is summarized as follows.

\subsubsection{Features of air shower induced radio pulses}

The radio emission of an air shower originates from the charged particles in the air shower, predominantly from the deflection of the electrons and positrons in the Earth's magnetic field. The emission is coherent if the thickness of the shower front (a few $\mathrm{m}$ ) is smaller than the wavelength of the radio emission ( $\widehat{=}$ radio frequencies $\lesssim 100 \mathrm{MHz}$ ). This leads to an amplification of the emission at those frequencies, and is the main reason why radio experiments operate typically below $80 \mathrm{MHz}$. Related to this frequency range is the time scale of the radio pulse which is in the order of $10 \mathrm{~ns}$, depending on the lateral distance to the air shower axis (see figure 2.4).

The amplitude (field strength) of the radio pulse is proportional to the number of electrons in the air shower, i.e., roughly proportional to the primary energy - like it is expected for a coherent emission mechanism. Furthermore, the amplitude depends on the geomagnetic angle (i.e., the angle between the air shower axis and the Earth's magnetic field), the lateral distance and possibly the zenith angle. However, the 
details of those dependencies are not fully known and under investigation, e.g., by analyzing the lateral distribution of the radio signal (cf., [34], and chapter 7).

Recently also the polarization of the radio signal became of interest. It is still being explored whether the polarization of the radio pulse can add valuable information to the reconstruction of the energy, mass and arrival direction of the primary particle. Several experiments measure, in addition to the arrival time and amplitude of radio pulses, their polarization, because this information at least helps to understand the emission process.

\subsubsection{Emission mechanisms}

The radio emission of air showers is a complex phenomenon. It is predominantly of geomagnetic origin, but also other mechanisms contribute to the total emission. It should be noted that splitting the total radio emission into contributions by different emission mechanisms will only be an approximation to nature. The most realistic description of the radio emission is obtained when the complete electromagnetic emission is calculated as a whole, e.g., by an end-point formalism [35]. This approach is followed by the Monte Carlo simulation program REAS3 [36], the latest version of REAS [37, 38].

Nevertheless, studying different emission mechanisms separately can help to understand the physical processes at work. Summing up the emission of individual mechanisms is an approach followed by the MGMR [39] model. This model agrees in first order with REAS3 calculations [33], which shows that the picture of individual emission mechanisms is a reasonable approximation. The deviation between REAS3 and MGRM is about a factor of $2-3$. It is currently under investigation up to which degree this deviation is due to the approach or its implementation, e.g., the simplified air shower model used in MGMR.

The following overview gives a summary of different emission mechanisms contributing to the total radio emission. Current experimental results are compatible with contributions from all mechanisms, as long as the dominant component is of geomagnetic origin.

1. Transverse currents due to the Earth's magnetic field [28]:

The (relativistic) electrons and positrons in the air shower experience a Lorentz force due to the Earth's magnetic field, and are separated from each other. This induces transverse currents in the air shower. Thus, the shower emits electromagnetic radiation, which happens to be predominantly in the range of radio waves below a few $100 \mathrm{MHz}$ (see figure 2.4). Today, it is assumed that this is the major contribution to the total radio emission by air showers [33]. The polarization of any geomagnetic radio emission is perpendicular to the Earth's magnetic field, i.e., mainly in the east-west direction.

2. Variation of the dipole strength of the air shower [40]:

The transverse currents in an air shower result in an electric dipole whose strength changes during the shower development. This leads to a radio emission, as if the shower were an emitting dipole antenna. Furthermore, the atmosphere transversed by the air shower is charged which should cause an additional, but smaller dipole-like radio emission [41]. 
3. Geosynchrotron effect [42]:

The electrons and positrons in their air shower are not only separated by the Earth's magnetic field, but also accelerated, i.e., they change their momentum. This should result in a synchrotron-like geomagnetic radio emission, because the tracks of the electrons and positrons are curved. However, recent theoretical studies revealed that this is only a minor contribution [33], and it is still under investigation by which degree it influences the total radio emission by air showers.

4. Variation of the net charge excess (Askaryan effect [27]):

In air showers, the number of electrons exceeds the number of positrons because of anti-particle absorption. The absolute amount of this charge excess changes during the shower development, as the total number of particles changes. Hence, this leads to a radio emission with radial polarization, like it is expected from any changing net charge (see also [33, 39]).

5. Cherenkov emission [43]:

When a charge is traveling in a medium faster than the speed of light in this medium, it is emitting Cerenkov radiation. Thus, also in air showers Cherenkov emission should occur, since the positive and negative charges in the shower are separated, and there are more electrons than positrons. Sometimes this Cherenkov-like radio emission is also referred to as Askaryan emission, since it is easy to confound and not well separated from the emission due to the net charge variation in the shower [35]. However, Cherenkov-like emission can only take place in media with a refractive index $n>1$. Thus, it could predominantly be of importance for particle showers in dense media. However, it is not clear, whether Cherenkov emission is negligible against the Askaryan effect which also occurs at $n=1$. Until now, radio emission in dense media has only been observed for accelerator beam induced showers.

6. Atmospheric electric fields:

Not only magnetic, but also electric fields in the atmosphere can accelerate the charged particles of the air shower and lead to an additional radio emission. During thunderstorm conditions $\left(E_{\text {atm }} \sim 10000 \mathrm{~V} / \mathrm{m}\right)$, this emission can be even stronger than the geomagnetic one [44]. It has still a measurable effect when the atmospheric electric field at ground reaches a strength of a few $1000 \mathrm{~V} / \mathrm{m}$ [31]. So far, no influence has been detected under normal weather conditions $\left(E_{\text {atm }} \sim 100 \mathrm{~V} / \mathrm{m}\right)$.

7. Molecular bremsstrahlung:

Recently also radio emission by molecular bremsstrahlung is investigated. Laboratory experiments with particle showers induced by an accelerator beam measured a partially coherent radio emission at $\mathrm{GHz}$ frequencies [45]. Thus, several experiments have started to search for air shower emission in the $\mathrm{GHz}$ range. If successful, this technique would have the advantage that air showers could be detected at large lateral distances, because molecular bremsstrahlung is emitted isotropically, and not mainly in forward direction like the geomagnetic emission. It is not yet clear, whether molecular bremsstrahlung has any significant influence on the radio emission at $\mathrm{MHz}$ frequencies. 
All emission processes are coherent at $\mathrm{MHz}$ frequencies because of the limited thickness of the air shower particle front (a few meters). Indeed, LOPES measurements show that the field strength of the radio pulse is (within errors) proportional to the primary energy [46], which proves that the emission is, at least to a large extent, coherent. Thus, any incoherent emission mechanisms can only play a minor role at LOPES energies $\left(\sim 10^{17} \mathrm{eV}\right)$.

At the moment, the research on the radio emission processes is focusing on the attempt to understand the radio emission as a whole, respectively, to figure out to which degree each of the mechanisms is contributing. This can be determined by studying properties of the radio pulses (e.g., pulse shape, polarization and lateral distribution) and their dependencies on different shower parameters (e.g., the geomagnetic angle, azimuth and zenith). Another way to disentangle the details of the emission process is to compare data with simulations based on certain models. If a model includes all of the processes leading to radio emission, its predictions should match the measured data. Great progress was recently made since it has become possible to compare the measured radio field strength for each LOPES event with predictions of REAS simulations [47].

To test improved simulations like REAS3, and to study also minor contributions to the radio emission, experiments must develop an increased level of precision. The present thesis covers several techniques required for precise measurements, for instance time calibration (see chapter 4), and noise treatment (see chapter 6). The success of the improved experimental techniques and the revised models becomes clear in the studies described in chapter 7 . For the first time, a simulation can predict measured radio data more or less correctly: lateral distributions of the radio field strength predicted by REAS3 are close to LOPES data. This gives rise to optimism that a detailed understanding of the air shower radio emission comes within reach. 



\title{
3. Radio Experiments for Air Shower Detection
}

\begin{abstract}
Already in the 1950's and 60's, several experiments discovered that cosmic ray air showers emit radio pulses in the $\mathrm{MHz}$ regime [29], that the emission is linked to the Earth's magnetic field, and that the radio amplitude is correlated with the energy of the primary cosmic ray particle. However, the limited electronics available at that time prevented to exploit the full potential of the radio detection method, and other methods, like the detection of fluorescence or Cherenkov light from air showers, have been more successful.

With the availability of fast digital electronics and computers, the radio detection method experienced a revival during recent years - starting with LOPES and CODALEMA in 2003 [30,48]. Also because of the success of those experiments, several air shower observatories are now extending their detectors by digital radio antennas. The most prominent among these extensions is AERA, a second generation digital radio antenna array at the Pierre Auger Observatory in Argentina.

This chapter begins with an overview on some major players in the field of digital radio detection of cosmic rays. Thereafter, the focus is on the LOPES experiment, since this thesis is mostly based on LOPES. Due to its small lateral extension and its noisy environment, LOPES is not suitable for precision cosmic ray physics. Nevertheless, it is an ideal test bed to explore the prospects of the digital radio detection technique. Thus, several methods developed at LOPES, for instance, the reference beacon (see chapter 5), are transferred to the next generation experiment AERA. Consequently, this chapter concludes with a presentation of the plans and status of AERA.
\end{abstract}

\subsection{Overview of modern radio experiments}

Nowadays, many experiments investigate the prospects of digital radio arrays for the detection of ultra-high energy particles, like cosmic rays and neutrinos. However, for the radio detection of particle showers in dense media, the proof of principle is not yet achieved. The situation for air shower detection is more advanced. Current experiments aim to optimize the radio detection technique, to understand the details of the emission mechanism, or to study how radio detectors improve the performance of hybrid experiments.

The following list gives a review on some important radio experiments. An overview of several modern experiments is available in the proceedings of the latest ARENA conference 2010, Nantes, France [49]. For a review of the historical experiments see reference [50]. 
LOPES LOPES is a digital radio interferometer at the Karlsruhe Institute of Technology, Germany, co-located with the KASCADE-Grande experiment [51]. LOPES successfully proofed that air shower properties can be reconstructed with digital radio interferometry [30], and plays a leading role in the development of the radio detection technique. The coincident operation of radio antennas with the particle detector array of KASCADE-Grande has been an essential ingredient for the success of LOPES. A detailed description of LOPES is presented in section 3.2. A recent overview over major LOPES results can be found in references [52] and [53]. In the frame of LOPES, the REAS program for Monte Carlo simulations of radio emission has been developed [36, 37].

LOFAR LOFAR is a digital low frequency interferometer for radio astronomy [ 54 , 55]. It consists of several antenna arrays, primarily in the Netherlands, but also in other European countries, whose measurements are combined in a subsequent analysis by digital beamforming. Among the key science projects of LOFAR are the detection of cosmic ray air showers and neutrino induced particle showers in the lunar regolith. LOPES (= LOFAR prototype station) was originally built to demonstrate that air showers can indeed be detected with LOFAR prototype hardware in a subsequent digital beamforming analysis.

CODALEMA Almost simultaneously with LOPES, also CODALEMA began to investigate the prospects of air shower detection with digital radio antenna arrays $[48,56,57]$. CODALEMA is located at the decametric radio observatory of Nancy in France. Compared to LOPES, it has the advantage of a radio quiet environment. Of disadvantage is the operation in coincidence with a relatively simple scintillator array for the detection of secondary air shower particles. Nevertheless, most of the progress in the field of digital radio detection of air showers in recent year was either made by CODALEMA or LOPES. Consequently, the experience and advantages of both experiments are combined for the second generation digital antenna array AERA.

AERA The Auger Engineering Radio Array (AERA) is a next generation digital radio array. It is currently under construction at the Pierre Auger Observatory in Argentina. By cross-calibration of radio measurements with surface particle detectors and fluorescence light detectors, AERA will investigate the performance of the radio technique for interferometric and non-interferometric reconstruction methods. For further information see [58] and section 3.3.

Tunka Tunka is a photomultiplier array for detection of Cherenkov light emitted by air showers [59]. Currently, a new hybrid approach is investigated with several radio prototype setups. A major goal is an $X_{\max }$ cross-calibration between radio and Cherenkov light measurements. For more details and first results of one of the radio prototype setups, see appendix A.

Yakutsk Probably the highest energy event detected with radio antennas $\left(E_{\text {est }}=\right.$ $2 \cdot 10^{20} \mathrm{eV}$ ) has been measured at the Yakutsk air shower array [60]. Although radio activities at Yakutsk came to a stop in 1989, they are currently restarted. Due to the 
coincident operation with the particle detector array, these new radio data might be useful to cross-check any future results of AERA with an independent experiment.

TREND The TIANSHAN Radio Experiment for Neutrino Detection (TREND) is being deployed on the site of the $21 \mathrm{~cm}$ array radio telescope, in XinJiang, China $[61,62]$. The full setup will consist of 80 antennas at an altitude of $2650 \mathrm{~m}$, in a radioquiet area of $4 \mathrm{~km}^{2}$ surrounded by mountains. Despite its name, TREND prototype antennas have been used only for air shower detection around $E \sim 10^{17} \mathrm{eV}$, so far. However, the main goal is the detection of horizontal showers induced by tau-neutrino reactions in the surrounding mountains [63]. This will only be successful if TREND can distinguish air shower induced radio pulses from RFI pulses. As most RFI pulses usually come from close to the horizon, this will be a challenging task. Thus, the radio quiet environment of TIANSHAN is an ideal testbed for this technique.

MSU Recently, radio data of the Moscow State University (MSU) array from the 1970's have been reanalyzed [64]. The MSU array consisted of Geiger-Müller counters above and under ground for $N_{\mathrm{e}}$ and $N_{\mu}$ measurements, respectively. In addition, 11 radio antennas were installed, operating in the frequency band from 30 to $34 \mathrm{MHz}$. The width of the radio lateral distribution has been studied for 85 events with high signal-to-noise ratio, at $E \approx 4 \cdot 10^{17} \mathrm{eV}$. The paper [64] claims a clear correlation between the mass sensitive $N_{\mathrm{e}} / N_{\mu}$ ratio and the width of the radio lateral distributions. The average $X_{\max }$ has been determined with a remarkable precision to $X_{\max }=655(13) \mathrm{g} / \mathrm{cm}^{2}$ by combination of particle and radio measurements with air shower simulations. This results is certainly in tension with recent radio experiments like LOPES. LOPES operates in about the same energy and lateral distance range, and features a larger bandwidth and event statistics. However, the precision of $X_{\max }$ measurements with LOPES is limited by systematic effects and noise, and is significantly worse than that claimed by [64] (cf. chapters 7 and 8).

Radio at IceCube and IceTop IceCube is a neutrino observatory at the south pole, detecting particle showers in ice by measuring their Cherenkov light with photomultipliers. To distinguish neutrino events from events caused by muons from cosmic ray air showers, a veto is provided by the IceTop air shower surface detector. It is planned to extend IceTop with surface radio antennas, for two reasons [65]: First, a radio measurement of the longitudinal shower development can improve the reconstruction accuracy of IceTop. Second, if the radio antennas cover a larger area than the surface detector, they can provide an additional veto for IceCube, even for more inclined showers which do not trigger the IceTop surface detector. Especially, using radio detection for vetoing is a promising approach, since a reasonable false trigger rate is tolerable as well as a bad reconstruction accuracy close to the threshold $\left(\sim 10^{16} \mathrm{eV}\right.$ for radio at IceTop).

Furthermore, in-ice radio detection of neutrino showers is under investigation with the planned Askaryan Radio Array (ARA) [66], and its precursor experiments RICE [67] and AURA [68]. Unlike cosmic rays, neutrinos do (almost) not interact in the atmosphere, and have a small probability for interaction in dense media, like the Antarctic ice shelf. However, for radio emission in dense media, not the geomagnetic 
deflection of shower particles, but the Askaryan effect is assumed to be the dominant emission mechanism. Yet, it has only been observed in laboratory experiments. Hence, it is interesting if ARA will observe any neutrino signals in nature.

ANITA The Antarctic Impulsive Transient Antenna (ANITA) is a balloon borne radio interferometer [69]. Its main purpose is the detection of ultra-high energy neutrinos which are assumed to emit an Askaryan radio pulse when interacting in the antarctic ice shelf. Yet, ANITA has no confirmed neutrino observation, but only candidate events. However, it claims the successful detection of geomagnetic radio pulses at energies $\sim 10^{19} \mathrm{eV}$. These radio pulses could be the radio signals of air showers which are reflected on the surface. Nevertheless, this result is under discussion because the ANITA frequency range of $300-1000 \mathrm{MHz}$ is beyond the frequency range predicted by geomagnetic models for the radio emission, like REAS or MGRM (cf. section 2.4). Currently it is investigated with REAS3, whether the coherent radio emission could extent up to those frequencies for close to horizontal showers [70].

Radio detection of neutrinos in dense media Besides ANITA and ARA, further radio experiments aim at neutrino detection in dense media by measuring Askaryan emission. ARIANNA [71] will use the antarctic ice shelf as detection volume. In addition, other dense media, for instance salt [72], are under investigation. An interesting approach is to use the lunar regolith as detection medium to search for particles at yet undiscovered energies $\gtrsim 10^{21} \mathrm{eV}$. This approach is followed both by ground based radio observatories, like LOFAR [73], and satellite observatories like LORD [74].

Acoustic detection of neutrinos In addition to radio detection of neutrinos in dense media, there is research going on to detect neutrino induced showers by sound. Therefore, several prototype experiments investigate hydrophone and microphone extensions of neutrino observatories in water and ice, like AMADEUS at ANTARES [75] and SPATS at IceCube [76], respectively. So far, no acoustic neutrino observation has been reported. Nevertheless, the methods used in acoustic and radio detection are similar, and both research fields mutually profit from developments in either of the fields.

Summarizing, significant progress has recently been made in the field of digital radio detection of cosmic ray air showers. Today we know that digital radio arrays are indeed suitable for air shower detection. However the question still remains, if the reconstruction accuracies of radio detectors can compete with established techniques like particle surface, fluorescence and Cherenkov detectors. Although this has not yet been demonstrated, AERA is expected to bring this proof in the next years, benefiting from the experience obtained at LOPES and other radio experiments. 


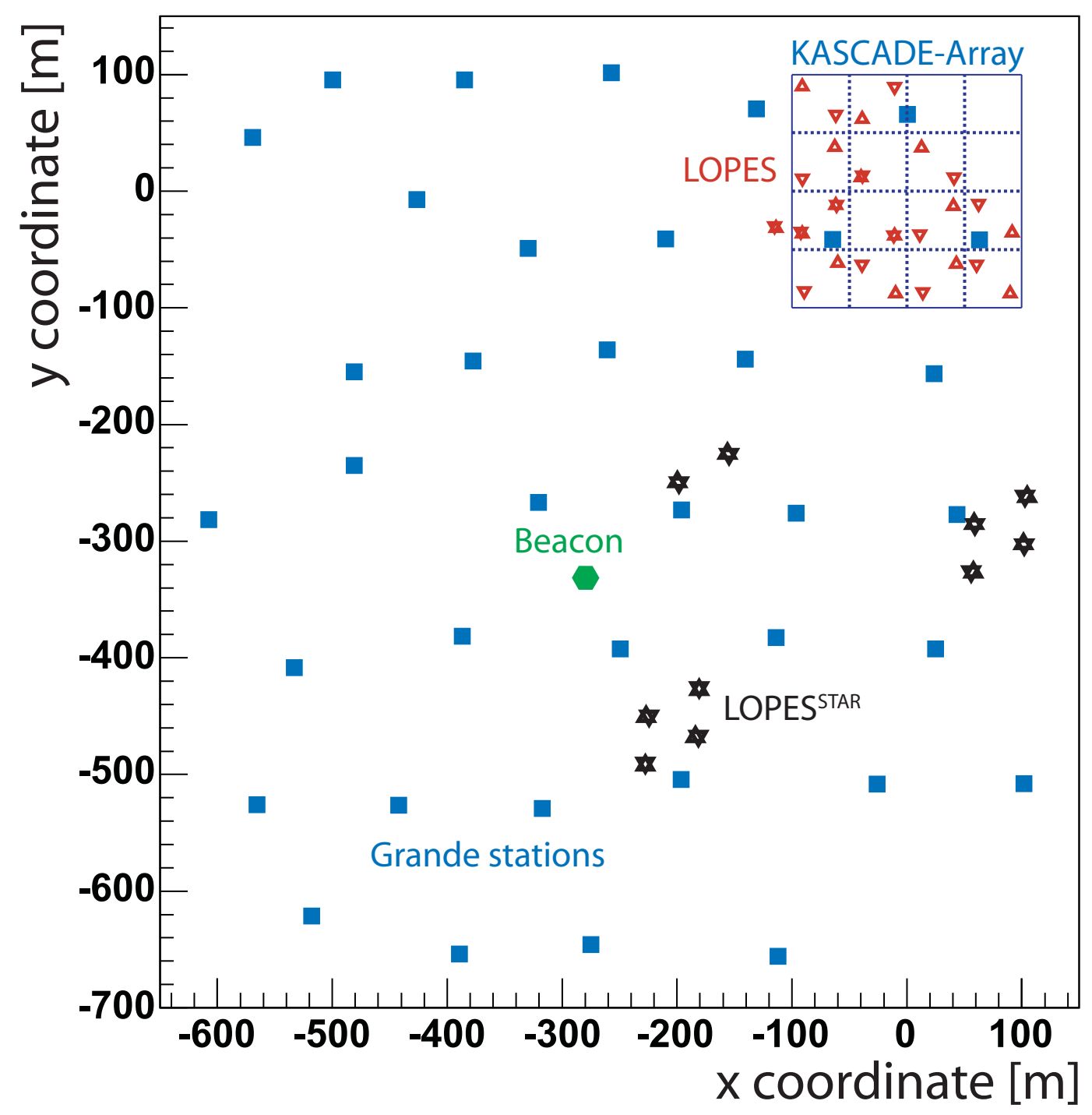

Figure 3.1.: Map of the LOPES experiment located inside of the KASCADE-Grande experiment at the Campus North of the Karlsruhe Institute of Technology, Germany. Shown is the configuration of the years 2007-2009: upward triangles mark east-west aligned antennas, downward triangles north-south aligned antennas, respectively. A star indicates an east-west aligned and a north-south aligned antenna at the same place. The upper right corner is the location of the KASCADE array, and the blue squares mark the location of Grande detector stations. Black stars indicate the position of LOPES ${ }^{\text {STAR }}$ antennas. The green hexagon is at the position of the reference beacon (see section 4.6). 


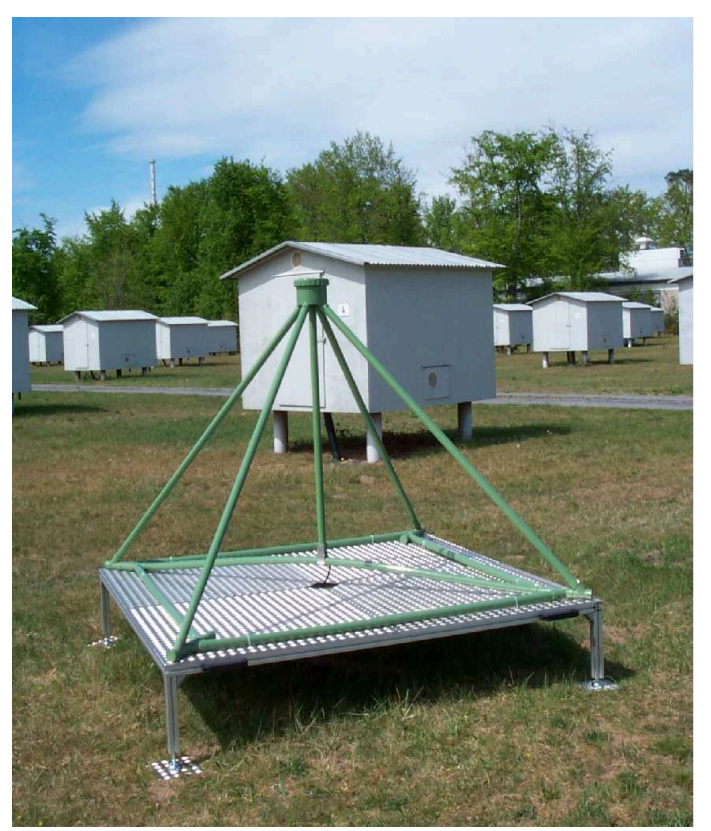

(a) inverted V-shaped dipole antenna

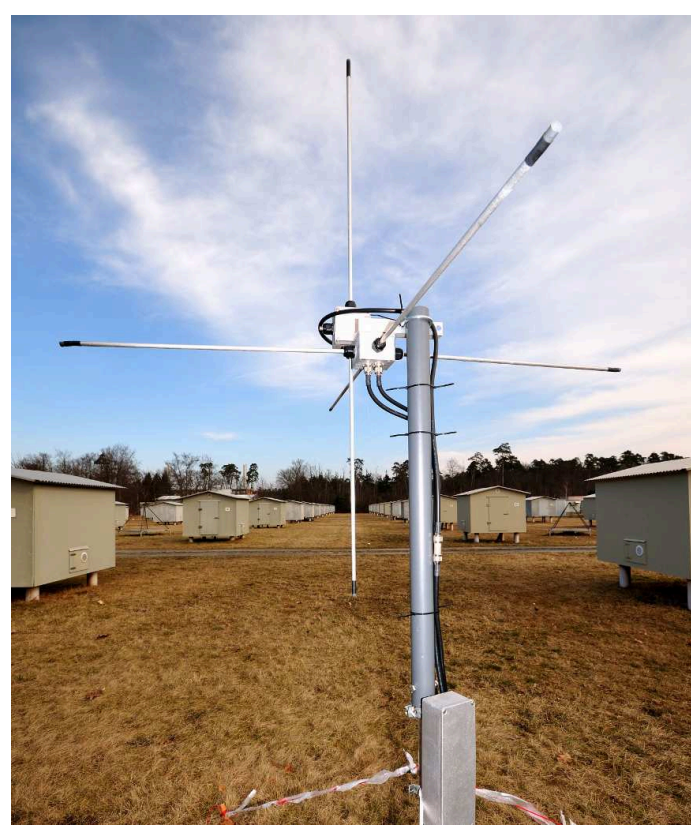

(b) tripole antenna

Figure 3.2.: Left: antenna type used in the LOPES 30 east-west and dual polarized setups. Right: antenna type used for the LOPES 3D setup.

\subsection{LOPES}

LOPES is a digital radio interferometer of 30 amplitude calibrated dipole antennas [77], placed inside the KASCADE-Grande experiment [78, 79], at the Karlsruhe Institute of Technology, Germany. It was built in 2003 as LOfar PrototypE Station, and was extended several times: additional antennas of different types have been added and tested, as well as an emitting reference beacon to provide a timing precision of about $1 \mathrm{~ns}$ for digital radio interferometer (see chapter 4 and [80]). The largest extension of LOPES is LOPES ${ }^{\text {STAR }}$ (Self Triggered Array of Radio detectors) which is devoted to the development of new antenna types, sophisticated hardware and self-triggering. Details can be found in [81] and [82], and will not be discussed in this thesis.

The following sections give a summary of the LOPES setup and the software used for analysis. For further reading see also references [83] and [47].

\subsubsection{Setup of LOPES}

LOPES started with 10 inverted V-shaped dipole antennas (see figure 3.2). To increase its sensitivity, LOPES was extended to 30 inverted V-shaped dipole antennas in 2005, which were all aligned in east-west direction. The east-west direction was chosen because the geomagnetic radio emission from air showers is dominantly polarized perpendicular to the Earth's magnetic field. In Karlsruhe it points almost exactly to the geographic north pole, with an inclination of $64^{\circ}$ [84]. At the end of 2006 , half of the antennas have been rotated by $90^{\circ}$ to be sensitive also to the north- 


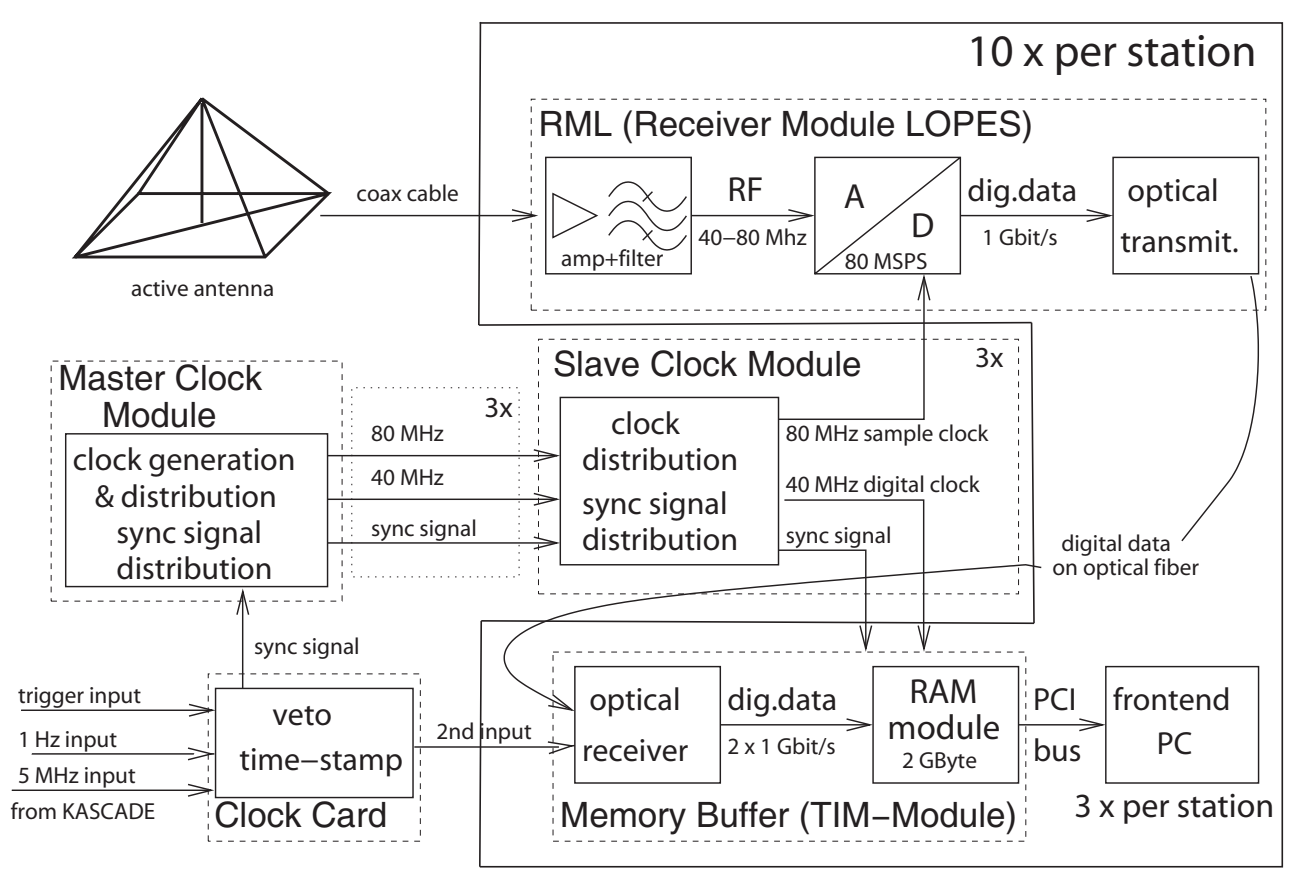

Figure 3.3.: LOPES hardware setup. Each antenna is connected to a receiver unit where the signal is amplified, filtered and digitized. The digitized signal is optically transmitted to a ring buffer at the DAQ board (TIM board) which is installed in a normal personal computer. A trigger signal (sync signal) initiates the read out of a certain fraction of this ring buffer and the transfer of the data to a central computer. All clock signals and the sync signal are centrally generated and distributed via cables in order to obtain exactly the same clock throughout the whole setup.

south polarized component of the air shower induced radio pulses [85]. Lately, in spring 2010, all 30 antennas have been exchanged for half-wave dipoles, each three of them forming a tripole antenna (see figure 3.2), in order to measure the polarization of the electric field directly at each antenna position [86].

In each setup, the same electronics is used for the digital read out (see figure 3.3), except for a slight change in the analog electronics which is accounted for in the analysis software. All antennas contain a low noise amplifier (LNA) receiving its supply voltage over the signal cable (RG 213) which connects each antenna to an analog electronics board. This board filters the signal to $40-80 \mathrm{MHz}$ with a bandpass filter to suppress RFI (radio frequency interference) from radio stations in the FM and short wave band. The board also contains the main amplifier, and feeds the signal to a digital computer board where it is sampled with a 12 bit ADC at a sampling frequency of $80 \mathrm{MHz}$.

Filtering the signal to a limited band-width is necessary to obtain the full information of the radio signal in this band with a limited sampling frequency. According to the Nyquist theorem [87], the sampling frequency has to be at least twice the bandwidth, a condition matched by LOPES. The full information of the recorded radio 
signal can be retained by up-sampling, which gives the correct interpolation between the sampled data points (see appendix B). For LOPES, in normal data acquisition mode, traces of $2^{16}$ data points per antenna are recorded simultaneously for each event, corresponding to about $0.8 \mathrm{~ms}$, with the trigger time roughly in the middle of the trace.

The read-out of LOPES is triggered by KASCADE-Grande, whenever KASCADEGrande detects a high energy cosmic ray event $\left(E \gtrsim 10^{16} \mathrm{eV}\right) \cdot{ }^{1}$ Then, the LOPES event data are stored simultaneously with the measurement of KASCADE-Grande, so that the KASCADE-Grande reconstruction can be correlated with LOPES measurements and used for event preselection. The trigger rate is about two events per minute, and the dead time in the order of one second, yielding about 3000 LOPES events per day.

LOPES and KASCADE-Grande event data are stored in several locations for later access. A monitoring of the noise level and the height of the continuous signal of a reference beacon allows for quick verification of system health [86]. In addition, the atmospheric electric field is measured at ground level for identification of thunderstorm events. Even atmospheric electric fields of a few $1000 \mathrm{~V} / \mathrm{m}$ as they can occur during normal rain, can result in slightly amplified radio pulses from air showers [31]. Hence, monitoring the atmospheric electric field is mandatory to exclude those periods from data analysis. The excluded periods typically amount to only $1.5 \%$ of the total LOPES operation time.

For later analysis, especially the results from the KASCADE and Grande arrays are used: KASCADE measures the number of electrons and muons of an air shower $\left(N_{\mathrm{e}}\right.$ and $\left.N_{\mu}\right)$ with scintillators in 252 stations placed on a grid of $200 \mathrm{~m} \times 200 \mathrm{~m}$ [78]. The Grande array measures the number of charged particles contained in an air shower $\left(\mathrm{N}_{\mathrm{ch}}\right)$ with scintillators placed in 37 containers on an area of about $0.5 \mathrm{~km}^{2}$ (see figure 3.1) [79].

\subsubsection{LOPES analysis pipeline}

Analysis of LOPES data aims to study the details of the emission mechanism of air shower induced radio pulses, and to explore the capabilities of reconstructing the direction, energy and type of the primary cosmic ray particles. This generally implies selecting LOPES events, reconstructing the properties of the measured radio pulse, and correlating the results to the KASCADE-Grande reconstruction obtained for the same events. For these steps, special software has been developed which allows a standard reconstruction procedure as described in the following paragraphs.

Since analyzing all triggered LOPES events would clearly exceed the available computer capacity, a preselection is necessary. Therefore, a software tool has been developed $^{2}$ which allows to select events for different criteria. E.g., only events passing certain quality cuts of the KASCADE-Grande reconstruction, and with a minimum estimated energy can be selected. This way, typically a list of up to a few thousand LOPES events is generated as input for the LOPES standard analysis

\footnotetext{
${ }^{1}$ The exact trigger condition is that either at least 10 of the 16 KASCADE clusters have triggered, or that the Grande hexagons 9, 10 and 13 have triggered which include 12 stations in the center of the Grande array [47].

${ }^{2}$ http://usg.lofar.org/doxygen/gen_eventlist_8cc.html
} 


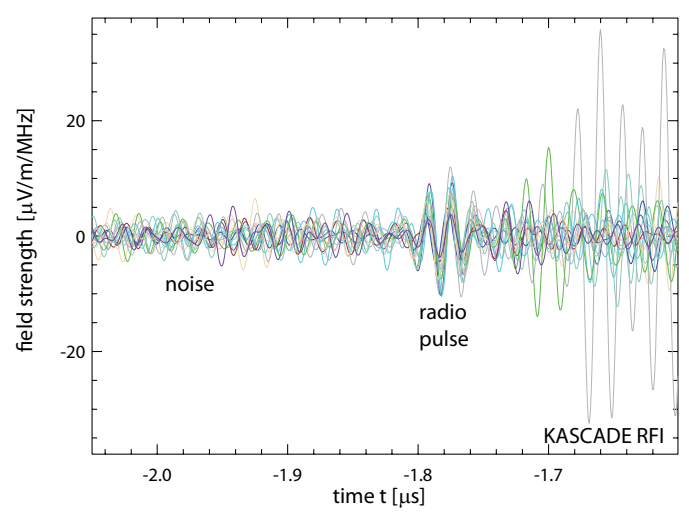

(a) individual antennas

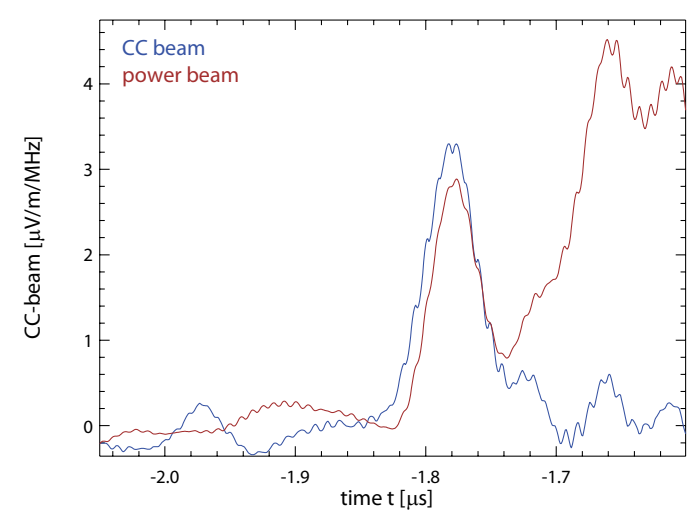

(b) cross-correlation and power beam

Figure 3.4.: Typical LOPES event: calibrated, up-sampled electric field strength in individual antennas (different colors) after correction for geometrical delays (left), the smoothed cross-correlation and power beam (right). The radio pulse induced by a cosmic ray air shower at $t \approx-1.8 \mu \mathrm{s}$ can clearly be distinguished from noise (and subsequent RFI caused by the KASCADE scintillators at $t \approx-1.7 \mu \mathrm{s}$ ), because only the air shower pulse is coherently detected in all antennas. The oscillation in the left plot is caused by the LOPES bandpass filter.

pipeline. For example, the event preselection used in chapters 7 and 8 consists of 3968 events.

The LOPES standard analysis pipeline ${ }^{3}$ is part of the open-source LOFAR software package [88]. It includes several steps for correcting instrumental effects (calibration), data conditioning, and determining parameters of the air shower induced radio pulse. Depending on the purpose of the analysis, not all steps have to be performed. The software package takes this into account by allowing to flexibly turn on or off certain steps of the analysis pipeline. The following list gives an overview of the most important steps, and a detailed description can be found, e.g., in references [83, 52].

\section{Calibration}

- conversion of ADC counts into voltage

- amplitude calibration (see [77])

- correction for cable and electronics delays (see section 4.4)

- correction for the pulse distortion by the bandpass filter (see section 4.5)

- verification of correct timing and fine tuning of the time calibration with the reference beacon (see section 4.6)

- application of the simulated antenna gain pattern

\footnotetext{
${ }^{3}$ http://usg.lofar.org/doxygen/call__pipeline_8cc.html
} 


\section{Data conditioning}

- application of a Hanning window to reduce aliasing caused by Fourier transforms, which are frequently performed during the whole analysis

- mitigation of narrow band radio frequency interference (RFI), by suppressing peaks in the frequency spectrum

- up-sampling by the zero-padding method (see appendix B and $[82,81]$ )

- correcting pulse heights for noise (see chapter 6)

\section{Reconstruction of radio pulse parameters}

- forming a cross-correlation $(\mathrm{CC})$ beam into the arrival direction of the radio pulse

- reconstruction of the lateral distribution of pulse amplitudes (see chapter 7)

- reconstruction of the pulse arrival time distribution (see chapter 8)

The capability to digitally form a cross-correlation beam with the electric field strength traces of all antennas makes LOPES a radio interferometer. A beam is formed by shifting the electric field strength traces of each antenna accordingly to the arrival time of the radio pulse (geometrical delay), in order that the radio pulse of each trace overlaps (see figure 3.4). After shifting the traces, they can either be added in square to form the power beam, or a cross-correlation between them is calculated to form the cross-correlation beam (CC-beam) [83]. A spherical radio wave front is assumed, corresponding to the simplification that the radio emission originates from one single point on the air shower axis (e.g., the shower maximum $\left.X_{\max }\right)$. The distance from the shower core to this point is the curvature radius of the radio wave front. The new result (see chapter 8 ), that pulse arrival time distribution of LOPES measurements and REAS3 simulations fit better to a conical wavefront, has not been implemented into the LOPES standard pipeline, yet.

In the LOPES analysis, the direction of the beam-forming and the curvature radius is optimized by a simplex fit. The initial direction for this fit is taken from the KASCADE-Grande reconstruction and the initial curvature radius from a scan, typically yielding a curvature radius of a few $\mathrm{km}$. The quantity maximized in the simplex fit is the height of a Gaussian fit to the cross-correlation beam, after smoothing it by block-averaging over $37.5 \mathrm{~ns}$. This time roughly corresponds to the pulse width which is determined by the bandpass filter. This procedure has been defined in [83]. It helps to distinguish the air shower induced radio pulse from subsequent RFI caused by the KASCADE scintillators, because in contrast to the radio pulse, the KASCADE RFI is generally incoherent. As the radio pulse is recorded about 100 ns earlier than the RFI from the KASCADE scintillators, it can also be distinguished by its arrival time. Thus, the chance of misidentifying the air shower radio pulse is negligible, except for events very close to the detection threshold. Low amplitude events have to be rejected by selection cuts on the signal-to-noise ratio of the cross-correlation beam and the fraction of correlated power, i.e., the ratio of the cross-correlation and power beam (cf. chapter 7.2). This is also important, as the 


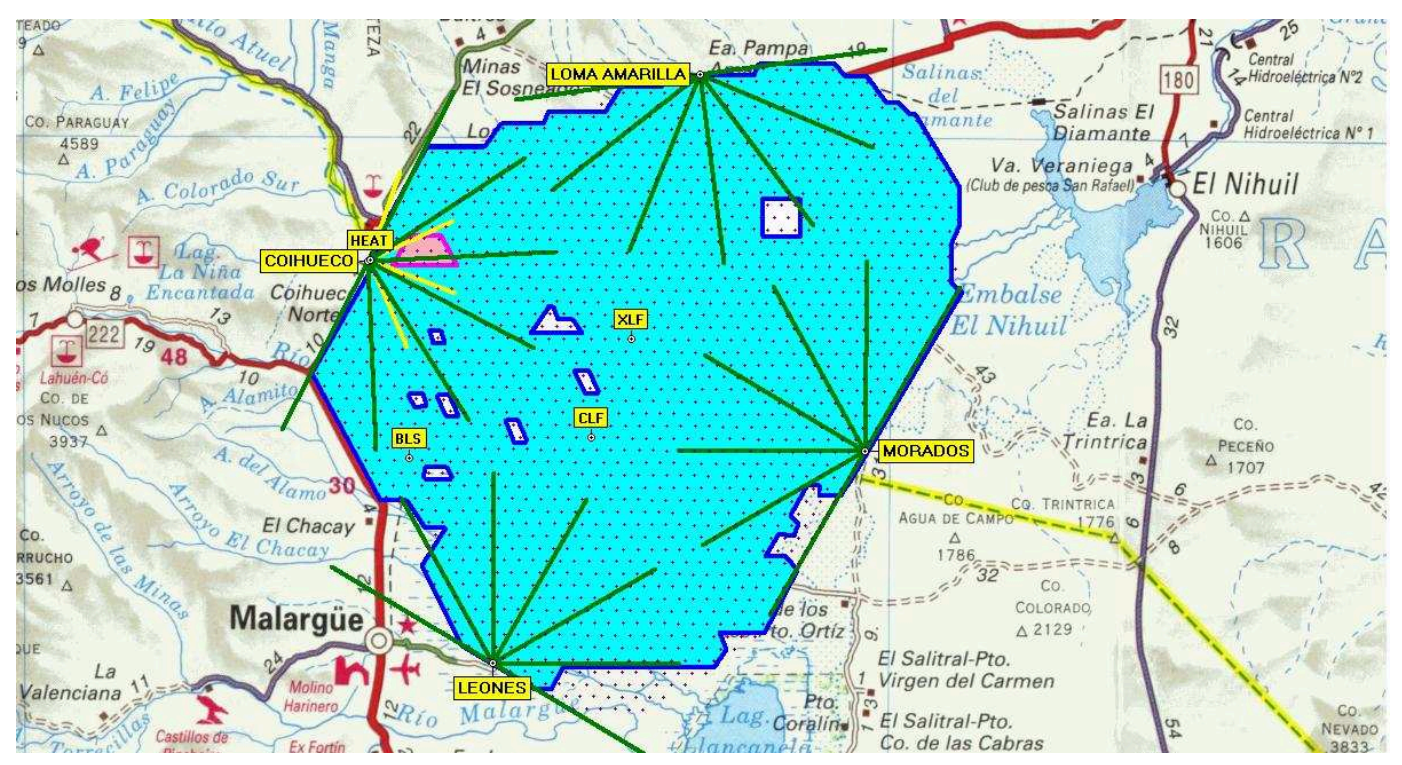

Figure 3.5.: Map of the Pierre Auger Observatory in Argentina. AERA is built at the infill array (pink), close to the Coihueco and HEAT florescence telescopes.

simplex fit is very sensitive to the initial values for events with low signal-to-noise ratios.

The cross-correlation beamforming exploits the assumption that all antennas coherently detect exactly the same pulse, which for a small array like LOPES is approximately but not completely true, because at least the amplitude of the radio emission decreases with lateral distance. The cross-correlation amplitude is used as a measure for the strength of the air shower induced radio pulse. In contrast to that, the power beam is a measure for the total power (air shower signal and background). Thus, the ratio between the power and the cross-correlation beam can be used to distinguish air shower signals form background, since the ratio should be close to 1 for radio pulses with a high signal-to-noise ratio. The error of the cross-correlation amplitude has not been studied in detail, since it is of minor importance for the analysis performed in this thesis. The error is estimated as the quadratic sum of a calibration uncertainty of $5 \%$ (originating from the influence of environmental effects, cf. [77]), the statistical error of the Gaussian fit to the smoothed cross-correlation beam, and a noise contribution which itself is estimated by the standard deviation of the cross-correlation beam in a time window before the radio pulse.

Concluding, cross-correlation beamforming is a useful tool to reconstruct the air shower arrival direction [89], the primary energy [46], and mass (see chapter 8). Moreover, the cross-correlation beam is crucial to select events for analyzing the lateral distributions of amplitudes and arrival times (see chapters 7 and 8). 


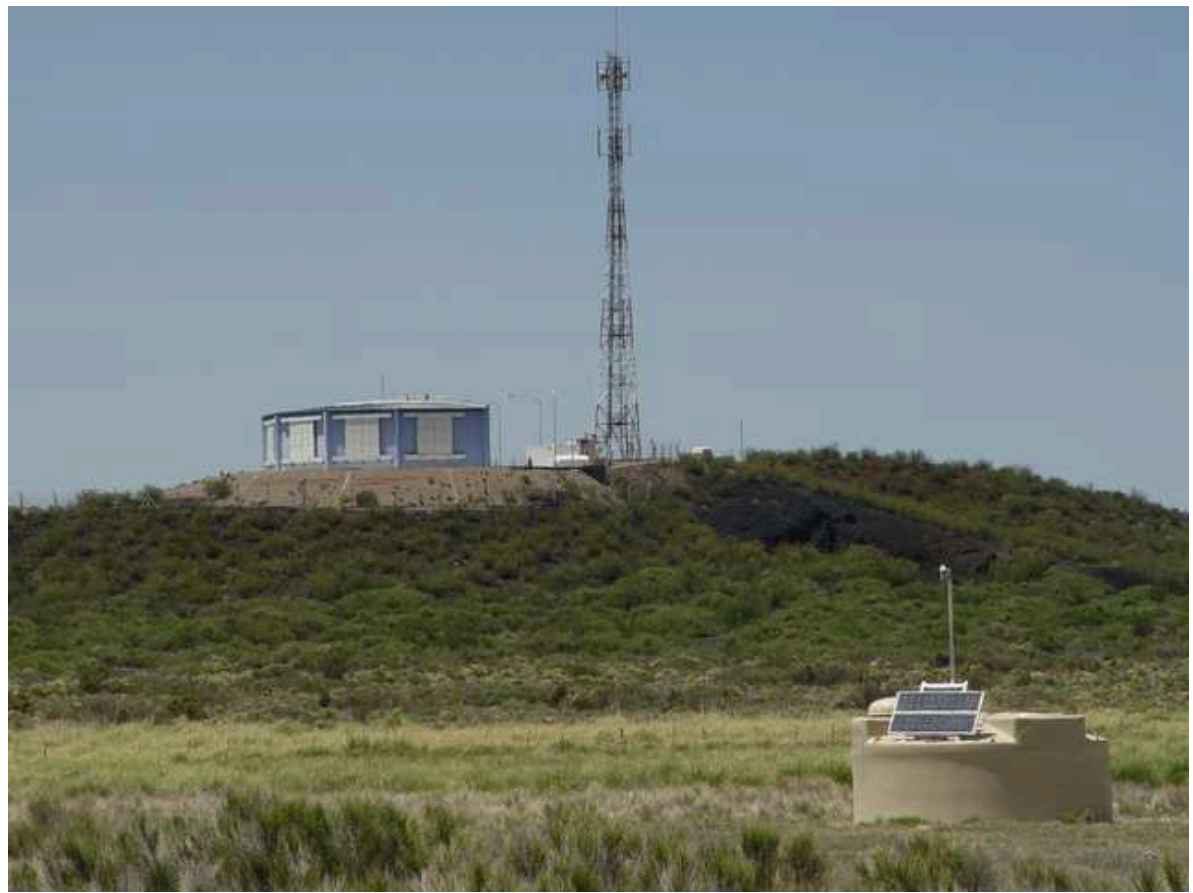

Figure 3.6.: Fluorescence telescope building and water Cherenkov detector of the Pierre Auger Observatory in Argentina.

\subsection{Radio detection at the Pierre Auger Observatory}

The Pierre Auger Observatory is the world's largest observatory for detection of cosmic ray air showers at highest energies $\left(\gtrsim 10^{18} \mathrm{eV}\right)[18]$. While the construction of a northern site is still in planning stage, the construction of the southern site in Malargüe, Argentina, has been finished in 2008 (see figure 3.5).

The Pierre Auger Observatory features a hybrid approach for the detection of cosmic rays. First, it detects air shower particles at ground level $(\sim 1400 \mathrm{~m}$ a.s.l. $)$ with 1600 water Cerenkov detectors at a spacing of $1.5 \mathrm{~km}$, covering an area of about $3000 \mathrm{~km}^{2}$ [90]. Second, in dark moonless nights, 24 telescopes in four buildings observe the fluorescence emission induced by air showers [91] (see figure 3.6). This combination of different measurement techniques allows a unique reduction of systematic uncertainties when reconstructing the energy and mass of the primary cosmic ray particles [92, 93].

Lately, the Pierre Auger Observatory started to be extended by several detectors to lower its energy threshold to about $10^{17} \mathrm{eV}$. These enhancements are concentrated in the same area to allow 'super-hybrid' measurements. They consist of high elevation telescopes (HEAT) [94], an infill array of surface detectors at a reduced spacing of $750 \mathrm{~m}$, and scintillators for muon detection which will be buried in the ground (AMIGA) [95]. Moreover, the installation of the Auger Engineering Radio Array (AERA) has been started at the same location. 


\subsubsection{Radio prototype setups}

As precursors of AERA, several prototype setups have already been deployed to explore the prospects of radio measurements of cosmic ray air showers at the Pierre Auger Observatory. These prototype setups use different antenna types and hardware, as well as different approaches for triggering and data transfer. This section summarizes shortly the properties of these prototype stations. More details can be found in references [96, 97, 98].

Close to the Balloon Launching Station (BLS), where the energy threshold of the $\mathrm{SD}$ array has been lowered by an additional detector, several radio stations have been installed. The exact configuration varies frequently. Here, only the setup of 2008 is described, when the measurements presented in chapter 5 were performed. At this time two different setups were running at the BLS:

- Three stations were equipped with SALLAs (short aperiodic loaded loop antennas [99]), and DAQ electronics developed within the scope of the LOPES STAR experiment. They operate in the frequency band between 40 to $80 \mathrm{MHz}$ in the second Nyquist domain, and measure the north-south and east-west polarization of the radio signal at each antenna station.

- The MAXIMA setup consisted of four autonomous, solar powered stations with wired LPDAs (logarithmic periodic dipole antenna [100]). They filter the radio signal to the band from 45 to $70 \mathrm{MHz}$, and sample it in the first Nyquist domain. Data are transfered from each station via a wireless link to a central computer in the BLS.

Both setups are equipped with a self-trigger, and can in addition be triggered by a clock in fixed intervals. This repeated, forced trigger allows studying variations of the radio noise level, and was used for the timing analysis of chapter 5. Furthermore, scintillators have been used for external triggering on air showers, for a part of the operation time. After a trigger, data are stored locally on hard disks, and occasionally transfered to Europe. Independently of the setup, all data can be analyzed with the Offline software framework described later in this chapter.

Moreover, there exists another prototype setup at the CLF, the RAuger experiment [98]. It consists of three autonomous radio stations with dipole antennas, and electronics developed within the CODALEMA experiment. RAuger was the first to show that self-triggering on the air shower radio emission is possible. However, the very simple threshold trigger used for RAuger will probably not be sufficient for AERA.

\subsubsection{AERA}

The Auger Engineering Radio Array is constructed in three phases, and is the next step for investigating the feasibility of radio detection on large scales. In its final phase, AERA will cover $10-20 \mathrm{~km}^{2}$ of the Auger enhancement area with about 150 radio detector stations (see figure 3.7). Phase 1 has already started in 2010 with 24 radio detector stations, a central radio station (CRS) for data acquisition, and a reference beacon for time calibration (see chapter 5 ). 


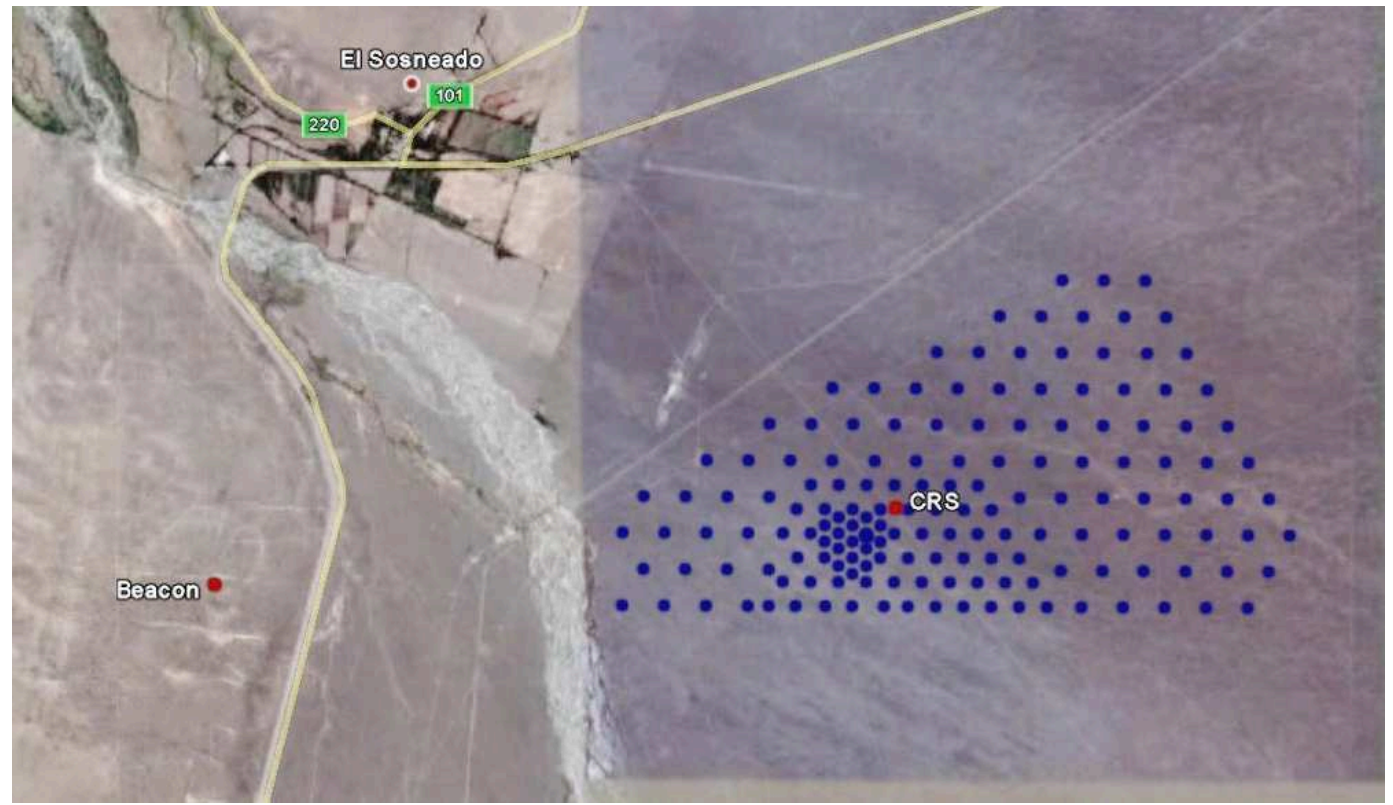

Figure 3.7.: Map of the Auger Engineering Radio Array (AERA). Antenna stations of the dense core close to the central radio station (CRS), and the reference beacon at the Coihueco telescope building are already installed (underlying map by Google Maps ${ }^{\mathrm{TM}}$ ).

AERA has several goals: First, it has to demonstrate that many technical challenges can be mastered, for instance, self-triggering on the radio pulse and digital interferometry with larger base lines. Second, the performance of the radio detection method for the reconstruction of arrival direction, energy and mass of the primary cosmic ray particle will be investigated and cross-checked with the reconstruction of other Auger detectors. While the goal for LOPES was to show that this reconstruction is in principle possible, AERA will reveal, whether the radio method can really compete with established methods, like the detection of fluorescence light. Third, AERA is expected improve the understanding of the radio emission by air showers in the energy range $\gtrsim 10^{17.5} \mathrm{eV}$. Finally, AERA measurements shall contribute to solve the questions of cosmic ray physics - provided that its reconstruction performance is sufficient.

Each AERA radio station consists of two antennas for the measurement of the north-south and east-west polarization, respectively, and a solar powered DAQ unit (see figure 3.8). For phase 1 of AERA, wired LPDAs are used as antennas, and for phase 2 and 3 an upgrade to another antenna type, e.g. a SALLA [99], is under investigation. The DAQ unit filters the radio signal to $30-80 \mathrm{MHz}$ and samples it in the first Nyquist domain. Restricting the signal band is necessary to reduce RFI from the short wave and FM radio channels. Each station triggers on the radio signal itself, and for background measurements at fixed times, too. After a trigger, each station transmits the radio data to the CRS. For phase 2 and 3 , also external triggering, e.g., by other radio stations is foreseen. 


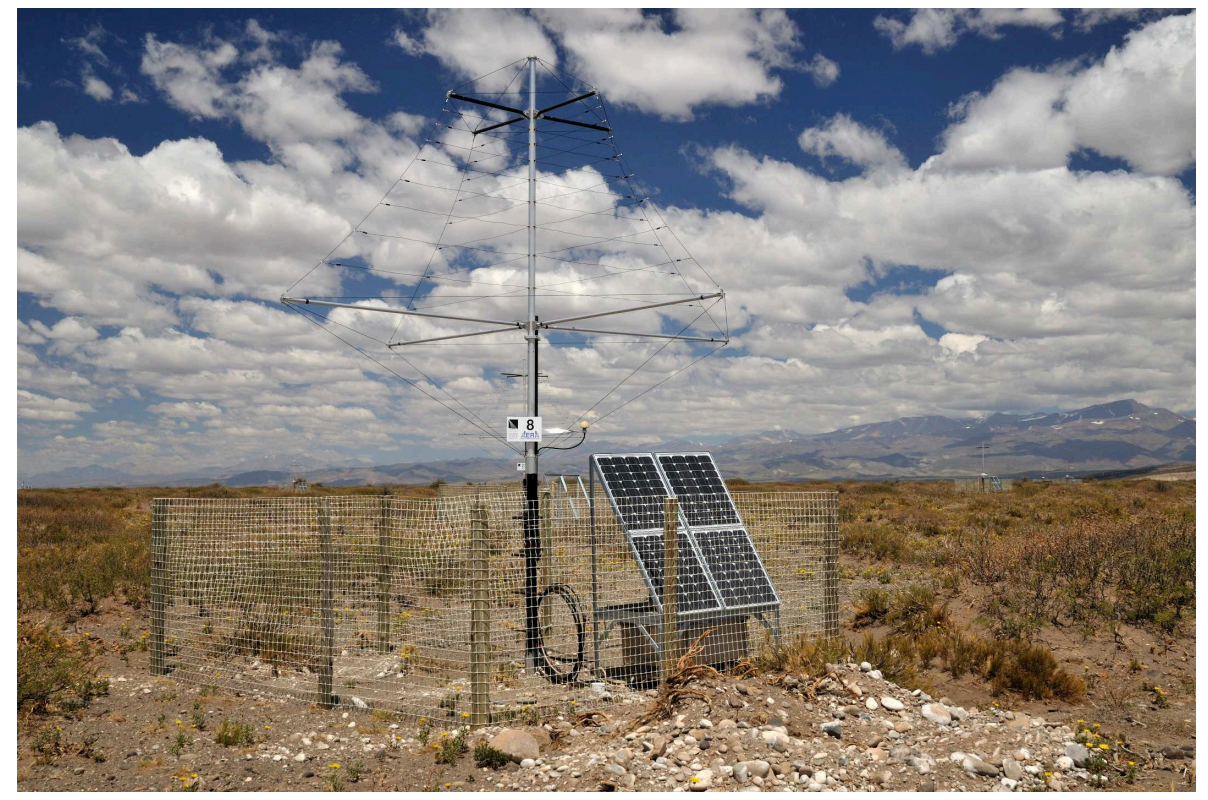

Figure 3.8.: AERA station with wired LPDA.

In the CRS, the data are stored, and monitoring of the data quality and system health takes places. Furthermore, the weather and especially the atmospheric electric field must be continuously monitored to detect thunderstorm-like conditions, since they can seriously affect the radio emission of air showers. After data transfer to Europe, the analysis can be performed offline.

\subsection{3. $\overline{\mathrm{Off}}$ line analysis software}

For the analysis of radio events obtained at the Pierre Auger Observatory, the existing Offline software package [101] has been extended [102, 103]. Offline has been developed to enable the evaluation of SD and FD measurements within one single software framework. It has a modular structure which allows to handle the reconstruction of data and simulations in a flexible way. The following paragraphs outline the functionality of Offline with respect to the radio data analysis.

Offline permits the read-in of different raw data formats, including the radio data of the BLS prototype setups, AERA, and radio simulations. Hence all radio data of the Pierre Auger Observatory can be analyzed with the same software, independently of the hardware which has been used for measurement. The different hardware configurations are taken into account during analysis by correction for instrumental properties, according to a database with the detector description. Finally, the results of the radio event reconstruction are stored in a special data format for later analysis and examination with an event display [104].

The Offline analysis pipeline basically consists of the same steps like the LOPES pipeline, and is even more flexible, as the order of modules is not fixed. Furthermore, it is easy to include additional features by programming additional modules or changing the implementation of a module. For example, one could easily exchange the algorithm of certain steps like RFI suppression or up-sampling. 
The main difference to the LOPES pipeline is that the Offline pipeline does not need initial input of SD or FD, like LOPES does from KASCADE-Grande. Instead, Offline aims to reconstruct the arrival direction and lateral distribution of the radio pulse by radio data alone. Of course, it also has the perspective to compare those quantities with FD and SD, or even performing a hybrid reconstruction, i.e., a reconstruction which combines several Auger detectors for the same event.

Another difference to LOPES is that the Offline analysis of AERA data can be noninterferometric by reconstructing the radio event parameters from the pulse arrival times and amplitudes, but without beam-forming. Benefiting from the more precise interferometric cross-correlation beam analysis of the same events, does not only require the appropriate software modules for Offline, but also the necessary timing precision of about $1 \mathrm{~ns}$. Preparing AERA for digital interferometry by installing a beacon system for time calibration, has been one of the achievements of this thesis (see chapter 5). 


\section{Time Calibration of LOPES}

Using LOPES as a digital interferometer, e.g., by forming a cross-correlation beam, requires precise knowledge of the antenna positions and the time when a radio pulse arrives at each antenna. The relative timing accuracy and precision required for digital interferometry is determined by the frequency band of LOPES $(40-80 \mathrm{MHz})$. It is in the order of $1 \mathrm{~ns}$ (see figure 4.1), which corresponds to a distance of $30 \mathrm{~cm}$, as radio waves travel with speed of light. The focus of this chapter is to explain by which methods a relative timing accuracy and precision of $\sim 1$ ns can be achieved. However, for pulse arrival time measurements at low signal-to-noise ratios, the uncertainty is dominated by noise and can be much larger than the calibration uncertainty, as it will be discussed in chapter 6 .

This chapter starts with a review how the amplitude calibration is performed for LOPES and explains why a different method is needed for time calibration. Then, after discussing the need for a relative timing accuracy in the order of $1 \mathrm{~ns}$, this chapter describes how the requirement is met with LOPES. A relative antenna positioning accuracy of much better than $30 \mathrm{~cm}(\widehat{=} 1 \mathrm{~ns})$ is achieved with differential GPS (Global Positioning System). For time calibration, a new method has been developed composed of three steps: measurement of the relative delays, correction for the dispersion of the bandpass filters, and permanent monitoring of the relative timing with a beacon. The beacon is a reference antenna which continuously transmits sine waves at known frequencies, and allows to detect variations of the relative timing between antennas, by measuring the phasing at these frequencies. ${ }^{1}$

\subsection{Amplitude calibration}

Absolute amplitude calibration for LOPES is performed with an external reference antenna, emitting a frequency comb with $1 \mathrm{MHz}$ spacing and fixed power at each frequency. A frequency dependent gain factor can be calculated for each antenna by comparing the emitted power of the reference antenna with the received power at each LOPES antenna, provided that the distance and angle between reference and LOPES antenna are known. The procedure is described in detail in [77] and [47], and shortly reviewed here. The present focus lies on the uncertainties, since they are important for the analysis of lateral distribution of the radio signal, which is discussed in chapter 7 .

For the amplitude calibration of a LOPES antenna, the reference antenna is placed with a wooden beam on a crane about $10 \mathrm{~m}$ above the LOPES antenna. During 2 minutes, 20 normal LOPES events are recorded with a forced, artificial trigger. The received power at each frequency emitted by the reference antenna is determined

\footnotetext{
${ }^{1}$ Most contents of this chapter have already been published in Nuclear Instruments and Methods A 615 (2010) 277-284 [80].
} 


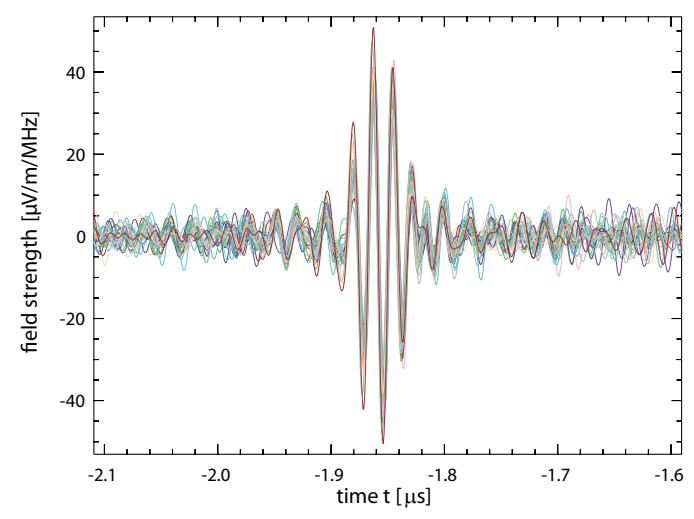

(a) individual antennas

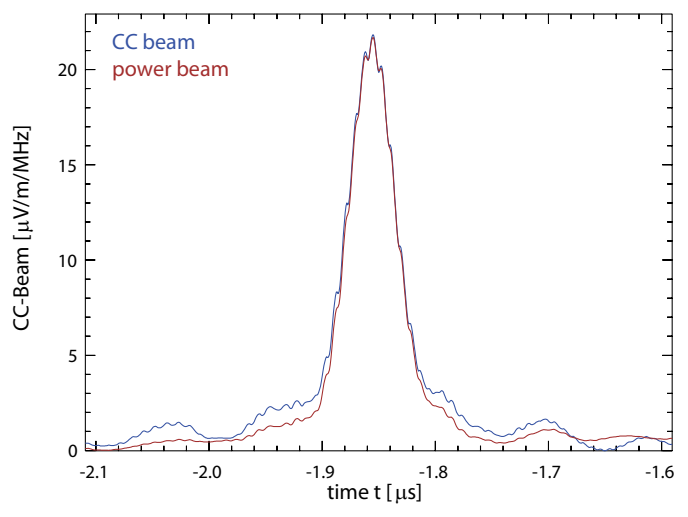

(b) cross-correlation and power beam

Figure 4.1.: Up-sampled electric field strength traces in individual antennas (different colors) of a 'perfect' example event after correction for geometrical delays (left), the cross-correlation and power beam (right). To be sensitive to the coherence of the radio pulse in all antennas, the relative timing accuracy has to be one order of magnitude smaller than the oscillation period of $\approx 17$ ns caused by the bandpass filter.

with the LOPES analysis software, and averaged over the 20 events. Then, the gain factor is calculated, assuming that the reference antenna has been exactly aligned with the LOPES antenna and at a constant distance to the LOPES antenna. The average distance during the 20 events is determined by differential GPS with an precision of a few $\mathrm{cm}$, but per-event variations of the distance, e.g., due to wind, are larger: they amount to a distance uncertainty of $\sim 25 \mathrm{~cm}$ per event. This corresponds to an uncertainty of about $2.5 \%$ for the amplitude which is small compared to the total uncertainty of $\sim 10 \%$. However, for time calibration, this distance uncertainty would correspond to a time uncertainty of almost 1 ns. This is the main reason, why a different method is needed for time calibration.

Other important uncertainties of the amplitude calibration are (relative uncertainties are given in brackets): the absolute scale of the amplitude of the emitted reference signal $(\sim 34 \%)$, the simulation of the antenna gain pattern which is used for evaluation of the calibration measurements $(7.5 \%)$, the uncertainty of the measured power $(2.5 \%)$, inaccuracies in the alignment of the antennas $(1 \%)$, and environmental effects $(4.5 \%)$ which have been studied by repeating the calibration measurements under different weather conditions. The calibration factors are generally stable within their uncertainties over the lifetimes of different LOPES setups. Still, a full amplitude calibration of all antennas is performed at least twice per year, since the calibration turned out to be also an excellent monitoring tool, e.g., to detect malfunction of amplifiers.

The calibration uncertainty of interest depends on the context: When comparing LOPES results to other experiments, the total calibration uncertainty is about $35 \%$ ( $\widehat{=} 70 \%$ in power), and is dominated by the uncertainty of the absolute scale. When comparing several LOPES events among each other, the absolute scale plays no role, and the calibration uncertainty is $\lesssim 10 \%$. When comparing amplitude measurements 
at different antennas for the same event, the uncertainty is even lower $(\sim 5 \%)$, because several effects like a change in environmental conditions or the amplifier gain occur on time scales of hours or more, but not per event. In every case, noise gives rise to an additional uncertainty on amplitude measurements, which at low signal-to-noise ratios can be even larger than the calibration uncertainty (see chapter $6)$.

Besides the distance uncertainty, the second reason that a different method is needed for time calibration is the unknown phasing of the reference antenna. The phase of the reference signal is unimportant for the amplitude calibration, but for time calibration it is important. It leads to pulse distortion by dispersion and can shift the arrival time of radio pulses (see section 4.5). In principle, the phasing of the reference antenna could be measured, but the effort would be large, and a precise time calibration can also be achieved by other means, as discussed in the following.

\subsection{Need for a precise time calibration}

For LOPES, the major need for a nanosecond relative timing precision and accuracy is digital interferometry, not angular resolution ${ }^{2}$. The angular resolution, respectively source location, of LOPES is limited to about $1^{\circ}$ [89], not because of the timing precision, but most likely because the spherical wavefront shape assumed in the LOPES standard analysis pipeline is only a vague approximation of the real wavefront shape (cf., chapter 8). Consequently, for LOPES, improving the timing precision is not expected to significantly improve the angular resolution. Instead, a timing precision in the order of $1 \mathrm{~ns}$ is a necessary requirement to enable the use of LOPES as a digital radio interferometer.

A timing precision which is at least an order of magnitude better than the period of the filter ringing ( $\sim 17 \mathrm{~ns}$ for LOPES), allows to perform interferometric measurements if the baselines of the interferometer are adequate for the angular scale of the observed source. The distance from the source of radio emission $(\approx$ the shower maximum) to the LOPES antenna array is typically several $\mathrm{km}$. This is much larger than the extension of the source region and the lateral extension of the array $(\sim 200 \mathrm{~m})$, so that the angular extension of the source is small. Hence, one expects that every antenna detects the same radio pulse just at a different time. Thus, LOPES should see coherent radio signals from air showers on ground, which has also been experimentally verified [30], and can be exemplarily seen in figure 4.1. This coherence is measurable, e.g., by forming a cross-correlation beam into the air shower direction [83]. The cross-correlation beam cannot only be used to reconstruct the direction, energy and primary mass, but also to distinguish between background (e.g., thermal noise and RFI originating from the KASCADE particle detectors) and air shower signals (cf. section 3.2.2).

The need for a timing precision in the order of $1 \mathrm{~ns}$ for the interferometric crosscorrelation beam analysis can be quantitatively verified by adding an additional and random timing uncertainty to each antenna, thus studying the influence on the reconstructed cross-correlation beam which is a measure for the coherence. This has

\footnotetext{
${ }^{2}$ Indeed, the requirement is a relative timing precision and accuracy of $\sim 1 \mathrm{~ns}$. To improve the readability, the text does not always explicitly refer to both terms.
} 


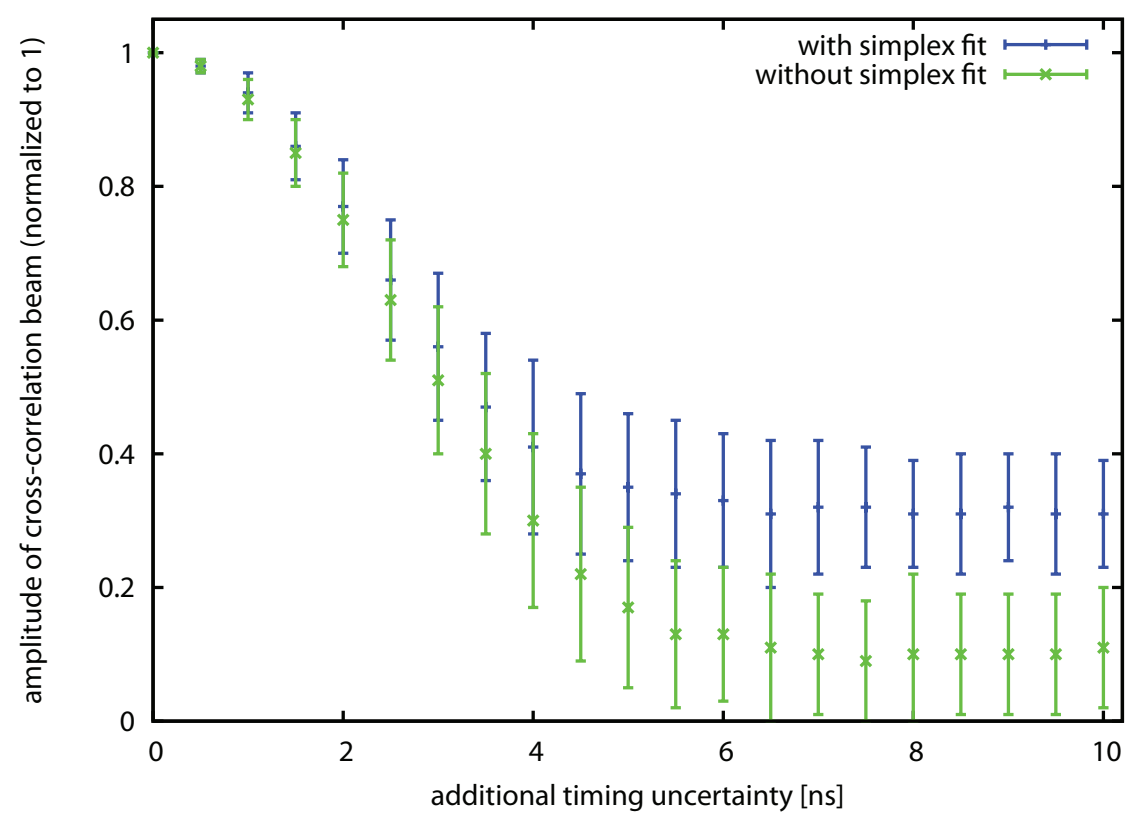

(a) for the event of figure 4.1 which has a CC amplitude of $20.9 \mu \mathrm{V} / \mathrm{m} / \mathrm{MHz}$

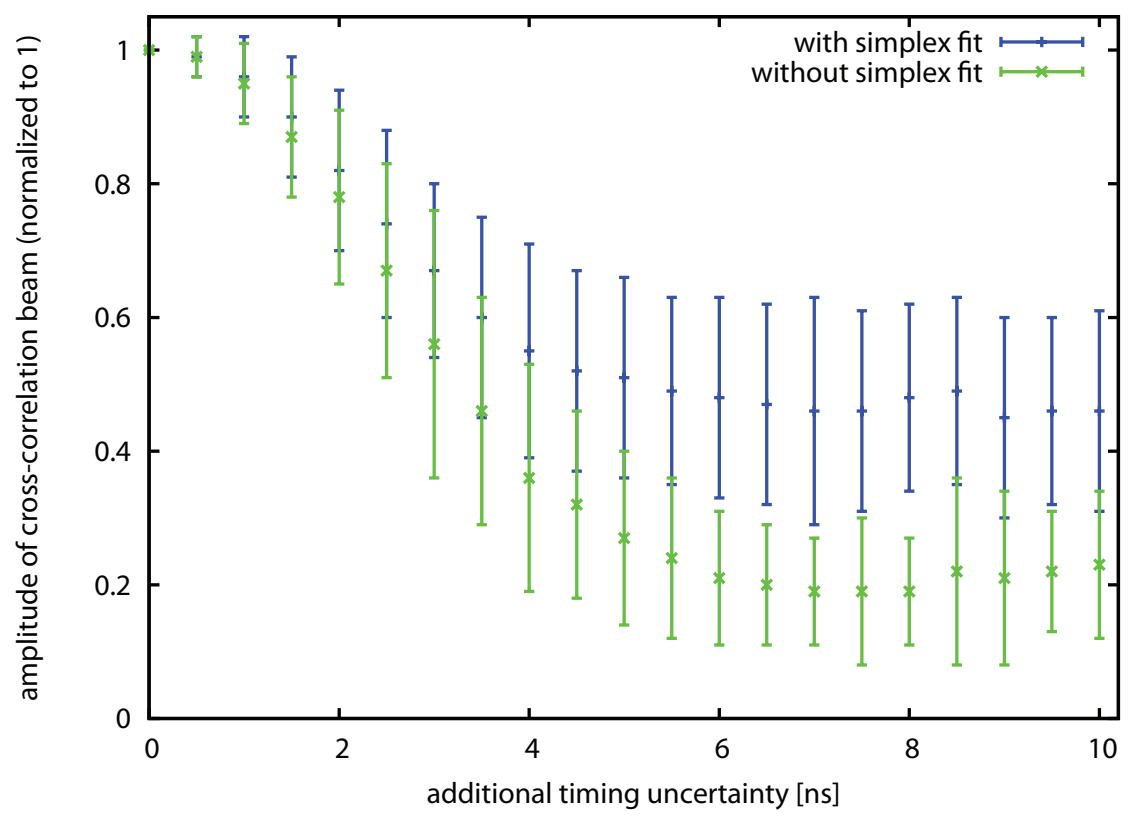

(b) for the event of figure 3.4 which has a $\mathrm{CC}$ amplitude of $3.2 \mu \mathrm{V} / \mathrm{m} / \mathrm{MHz}$

Figure 4.2.: Influence of an additional Gaussian timing uncertainty on the amplitude of the cross-correlation beam: The x-axis shows the width of the Gaussian distribution of the timing uncertainty added to each antenna. For both events, 100 repetitions have been performed for each timing uncertainty: the plot shows means and spread (standard deviation). The simplex fit of the standard analysis pipeline optimizes the beamforming direction and radius of curvature. For large timing uncertainties, this leads to an enhanced amplitude, since the fit always finds a random coherence. 
been done for three events which differ by the amplitude of the cross-correlation beam. The traces of each antenna have been shifted by an additional time taken from a Gaussian random distribution (see figure 4.2). The amplitude of the crosscorrelation beam decreases significantly when the added uncertainty is larger than $1 \mathrm{~ns}$. For uncertainties $\gtrsim 5 \mathrm{~ns}$ the amplitude is not reduced further. If the direction and curvature radius is fixed to the values reconstructed without an additional timing uncertainty, and not optimized by the simplex fit, the calculated cross-correlation amplitude levels off at $\sim 10-20 \%$ of the initial value. To reproduce the analysis of events more realistically, the direction and curvature radius has been optimized each time by the simplex fit of the standard analysis, which always finds a random correlation between some antennas. In this case, the cross-correlation amplitude is about one third of the initial value for the event of figure 4.1. This event has a relatively high cross-correlation amplitude of $20.9 \mu \mathrm{V} / \mathrm{m} / \mathrm{MHz}$. The cross-correlation amplitude can reach almost one half of the initial value due to random coherence, for two events studied with a mediocre cross-correlation amplitude between 3 and $5 \mathrm{\mu V} / \mathrm{m} / \mathrm{MHz}$ (only one of them is shown in figure 4.2).

Since most of the LOPES events are close to the noise and have a low crosscorrelation amplitude, reconstructing the cross-correlation beam correctly is essential. First, a reduced amplitude can lead to a signal-to-noise ratio below the detection threshold and a none-detection of real events. Second, there is a considerable risk of false events due to the random correlations, which is encountered by a quality cut on the correlated part of the pulse power (i.e., amplitude of the cross-correlation beam/ amplitude of the power beam $\geq 80 \%$ ).

Another motivation for a precise timing are foreseen studies of the polarization of the electric field $[85,86]$ : Different models for the radio emission of air showers can, among others, also be tested by their predictions on the polarization of the radio signal (e.g., REAS [105, 106, 36] predicts predominantly linear polarization of the electric field in a direction depending on the geometry of the air shower [38]). The capability of any antenna array to reconstruct the time dependence of the polarization vector at each antenna position, and thus to distinguish between linear and circular polarized signals, depends strongly on the relative timing accuracy between the different polarization channels of each antenna.

Finally, a precise timing is required to reconstruct the distribution of pulse arrival times (see chapter 8): According to simulations, the distribution of the pulse arrival times should contain information about the mass of the primary cosmic ray particle [107]. Only a precise relative timing, even between distant antennas $(\sim 200 \mathrm{~m}$ for LOPES), can enable us to measure the shape of the radio wave front in detail.

Stable clock signals for the DAQ electronics and the trigger signal of LOPES are distributed via cables (cf., figure 3.3). Consequently, the time calibration is reduced to the measurement of the electronics and cable group delays, their dependence on the frequency (dispersion), and their variations with time. Originally, the delays were measured with the radio emission from solar burst events, and their variations were monitored by measuring the phasing of a television signal [30, 83]. From the end of 2007 on, this method could not be applied anymore, because the television transmitter was switched off, and the solar cycle reached its minimum.

Meanwhile, new methods for the time calibration have been developed which do not depend on external sources out of our control. Namely, the delays are measured 
Table 4.1.: LOPES coordinate system

\begin{tabular}{lr}
\hline Base of GPS system & WGS 84 \\
Latitude & $49^{\circ} 05^{\prime} 59.10793^{\prime \prime} \mathrm{N}$ \\
Longitude & $8^{\circ} 26^{\prime} 10.71090^{\prime \prime} \mathrm{E}$ \\
Ground Northing & $36.11 \mathrm{~m}$ \\
Ground Easting & $-84.535 \mathrm{~m}$ \\
Zero height & top of concrete plate of KASCADE huts \\
Orientation of x coordinate & towards North \\
Orientation of y coordinate & towards East \\
Orientation of z coordinate & upwards \\
\hline
\end{tabular}

with reference pulses emitted at a known time, and corrected for the dispersion of the analog electronics. In addition, an emitting reference antenna, a beacon, has been installed which continuously transmits two narrow band sine signals to monitor variations of the delays with time.

These three methods for calibration and monitoring of the timing are combined to achieve a timing precision and accuracy in the order of $1 \mathrm{~ns}$ for each event measured with LOPES. Nevertheless, these methods are in principle independent from each other. E.g., at AERA a beacon is used to continuously monitor and improve the relative timing (see chapter 5), but electronics and cable delays will be measured with different methods.

\subsection{Measurement of antenna positions}

Digital radio interferometry does not only require a precise time calibration, but for the same reasons, also a precise measurement of the antenna positions. Uncertainties of the antenna positions can be translated into a timing uncertainty by dividing with the speed of light. Hence, they must be $<30 \mathrm{~cm}$ ( $\widehat{=} 1 \mathrm{~ns}$ ).

The positions of the LOPES antennas have been measured with differential GPS, in a coordinate system (see table 4.1) pointing towards geographic North, which deviates from the magnetic North by a about $1^{\circ}$ (see e.g., [84]). This magnetic declination has changed slightly over the run time of LOPES, and is ignored in the calibration and analysis, as it is small compared to other systematic errors. For instance, the alignment of the LOPES antennas towards magnetic North has been checked with a binocular with an integrated compass to be accurate to $\pm 2.1^{\circ}$ (mean deviation between the orientation of all 30 LOPES antennas and magnetic North, measured on 07 May 2009).

Already for the LOPES 30 east-west setup, antenna positions were determined with a Promark $2^{\mathrm{TM}}$ differential GPS system (see appendix C). However, the accuracy had not been definitely known, except that the statistical error of the relative positions was in the order of several $\mathrm{cm}$. For the dual-polarization setup, the position measurement was repeated twice with the more accurate Promark $3^{\mathrm{TM}}$ GPS system. By comparing those two measurements with each other, the uncertainties of the antenna positions could be estimated. They are below $1 \mathrm{~cm}$ for the $\mathrm{x}$ and $\mathrm{y}$ position and about $1.5 \mathrm{~cm}$ for the $\mathrm{z}$ position (mean deviation between two GPS measure- 
Table 4.2.: Conformance between LOPES and KASCADE coordinate systems

\begin{tabular}{lr}
\hline Angle between KASCADE and LOPES coordinate systems & \\
GPS measurement & $(15.25 \pm 0.04)^{\circ}$ \\
Calculation for KIT Campus North grid & $15.23^{\circ}$ \\
Evaluation of Google Earth $^{\text {TM }}$ image & $(15.18 \pm 0.08)^{\circ}$ \\
\hline Estimated deviation between centers of coordinate systems & \\
Deviation for x coordinate & $\approx 0.2 \mathrm{~m}$ \\
Deviation for y coordinate & $\approx 0.2 \mathrm{~m}$ \\
Deviation for z coordinate (height) & $\lesssim 0.05 \mathrm{~m}$ \\
\hline
\end{tabular}

ments of the same antennas, at different days). Moreover, a systematic uncertainty of about $5 \mathrm{~cm}$ has to be considered, as there is a certain margin in assembling the LOPES antennas. Still, the total position uncertainty is well below the requirement of $<30 \mathrm{~cm}$.

In addition to the relative positioning of the antennas to each other, also the conformance of the LOPES and KASCADE coordinate system matters. This conformance is not required for digital interferometry, but is necessary to correctly reconstruct the shower axis and shower plane. This is important for the analysis of lateral distributions of pulse amplitudes and arrival times (see chapters 7 and 8).

The LOPES coordinate system has the same center as the KASCADE coordinate system, and is pointing towards geographic North. On the other hand, the KASCADE system is aligned with the grid of the KIT Campus North which does not point to geographic North but has an offset of about $15.25^{\circ}$. Furthermore, the LOPES system is left-handed as $x$ is pointing towards North and $y$ towards East, but the KASCADE system is right-handed. Any error originating from the conversion of the shower core from the KASCADE into the LOPES coordinate system should be smaller than the uncertainty of the shower core itself (typically $4-7 \mathrm{~m}$ in the energy range of interest for LOPES). Otherwise, the conversion into the LOPES coordinate system would introduce a significant error for the determination of lateral distances to the shower axis.

Consequently, the conformance of the coordinate centers was checked, and the angle between the LOPES coordinate system and the KASCADE system had been determined with sufficient precision $\left(\lesssim 0.1^{\circ}\right.$, which corresponds to a lateral distance uncertainty of $\sim 1 \mathrm{~m}$ for a shower core at $\sim 500 \mathrm{~m}$ distance). Therefore, the centers of the LOPES and KASCADE coordinate systems have been matched by comparing calculated antenna positions in the KASCADE system with GPS measurements and manual measurements of antenna heights, because the uncertainty of GPS measurements alone is too large (a few meters). Furthermore, the angle between the LOPES and KASCADE systems has been evaluated by different methods, all of them yielding the demanded precision (see table 4.2).

Summarizing, the centers of the LOPES and KASCADE coordinate systems are equal within a remaining deviation of $\sim 0.3 \mathrm{~m}$, which is an order of magnitude better than the typical shower core uncertainty. The more important relative uncertainty 


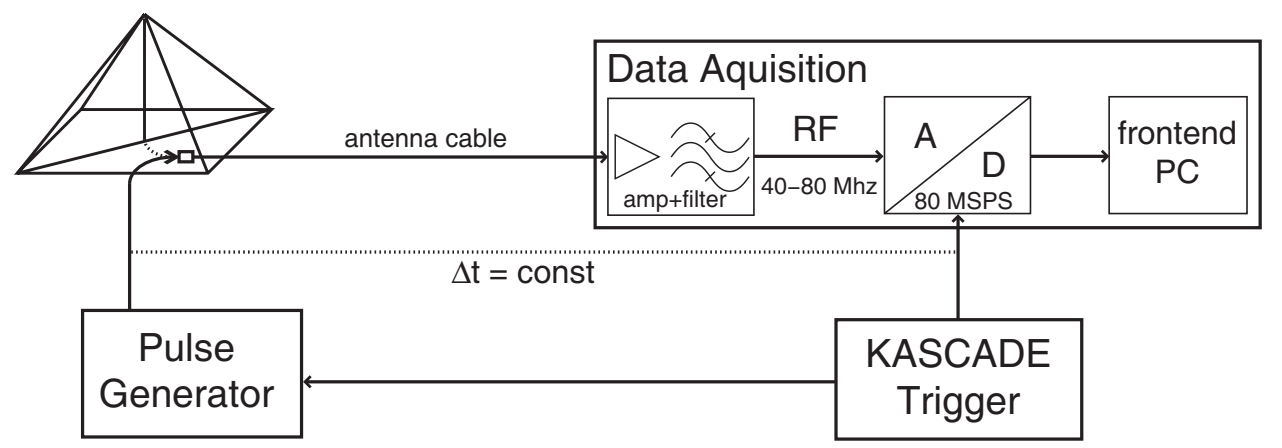

Figure 4.3.: Setup for the delay measurements: the coaxial cable from the LOPES antenna is disconnected from the antenna and connected to a cable from the pulse generator instead which is triggered simultaneously with the DAQ electronics.

of the antenna positions to each other is about $6 \mathrm{~cm}$ which corresponds to $0.2 \mathrm{~ns}$, and clearly fulfills the requirement of about $1 \mathrm{~ns}$ for digital interferometry.

\subsection{Measurement of the relative delays}

For LOPES, as a digital radio interferometer, mainly the relative timing between the different antennas is of importance, and the absolute event time has only to be known roughly to combine the LOPES events with the corresponding KASCADE-Grande events. Thus, the determination of the pulse arrival times at each antenna, and therefore the measurement of the delays, is most important on a relative basis. The delay of each channel (antenna and its analog electronics) is different, e.g., because different cable lengths are used.

The absolute delay $\tau$ of a channel is defined as the time interval between the arrival time $t_{0}$ of a radio pulse at an antenna and the time $t_{\mathrm{t}}$ when it appears in the digitally measured trace: $\tau=t_{\mathrm{t}}-t_{0}$. The more important relative delay $\Delta \tau_{\mathrm{m}, \mathrm{n}}$ between two antennas $\mathrm{m}$ and $\mathrm{n}$ is the difference between the absolute delays of these antennas: $\Delta \tau_{\mathrm{m}, \mathrm{n}}=\tau_{\mathrm{m}}-\tau_{\mathrm{n}}$. Using solar bursts, all relative delays $\Delta \tau_{\mathrm{m}, \mathrm{n}}$ could be determined directly. But due to the absence of solar bursts in the solar minimum, a different method was used from 2007 on.

Measuring the delays of different antennas not relative to each other, but with respect to a common reference time $t_{\text {ref }}$ is equivalent if the difference $t_{\text {ref }}-t_{0}=$ const, is the same for all antennas. These delays $\tilde{\tau}$ measured with respect to $t_{\text {ref }}$ are related to the absolute delays by

$$
\tilde{\tau}=t_{\mathrm{t}}-t_{\mathrm{ref}}=\tau-\left(t_{\mathrm{ref}}-t_{0}\right) .
$$

The relative delays $\Delta \tau_{\mathrm{m}, \mathrm{n}}$ can be easily derived from the measured delays $\tilde{\tau}$ by

$$
\Delta \tau_{\mathrm{m}, \mathrm{n}}=\tau_{\mathrm{m}}-\tau_{\mathrm{n}}=\tilde{\tau}_{\mathrm{m}}-\tilde{\tau}_{\mathrm{n}} .
$$

For each antenna, the measurement of the delay $\tilde{\tau}$ is performed as follows: The signal cable is disconnected from the antenna and connected to a pulse generator in- 


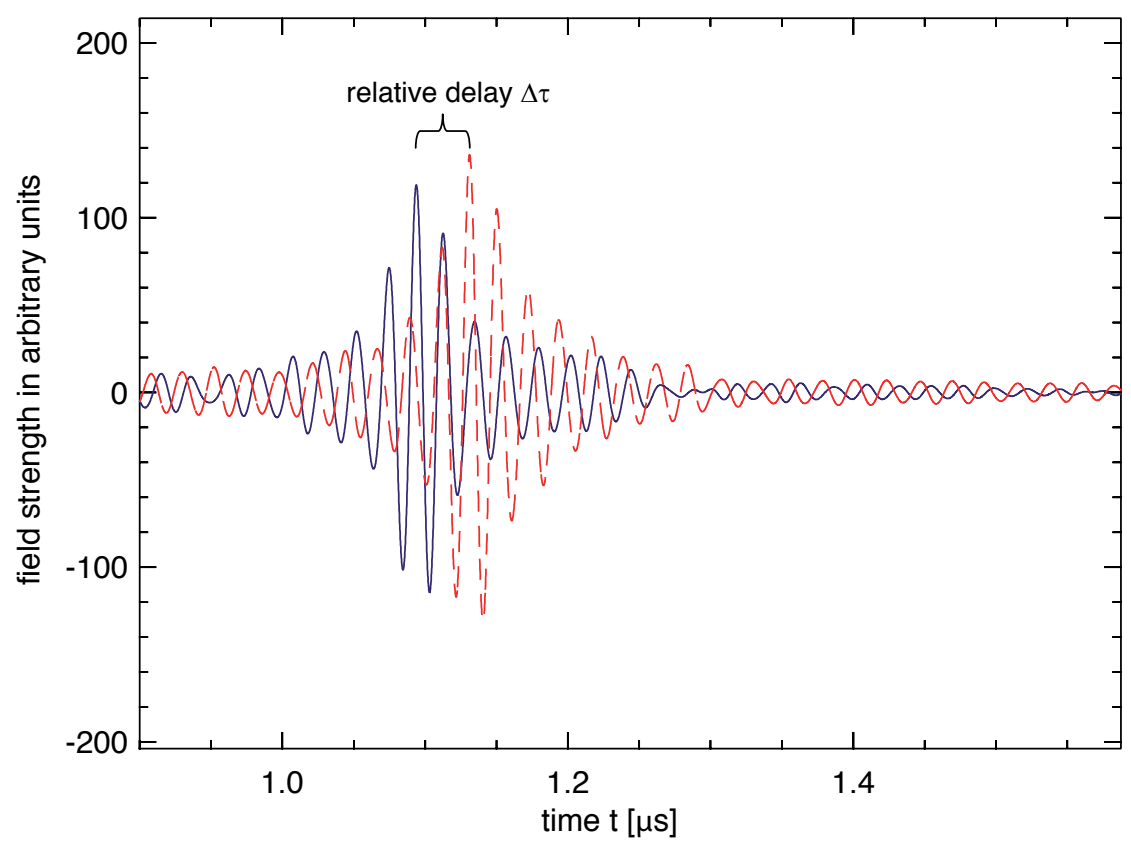

Figure 4.4.: Example for the delay measurement: the relative delay between two antennas (solid blue and dashed red) can be measured as the difference of the times when a calibration pulse is received, which has an amplitude of about $1 \mathrm{~V}$ and is fed directly into the antenna cables. The relative delay is mainly caused by different cable lengths.

stead, which emits a short calibration pulse at a fixed time after a normal KASCADEGrande trigger (figure 4.3). As reference time $t_{\text {ref }}$ we define the zero point of the LOPES trace (i.e., $t_{\text {ref }}=0$ ), which is determined by the KASCADE-Grande trigger, because it starts the LOPES read out. As it simultaneously triggers the pulse generator of the delay measurement, the condition $t_{\text {ref }}-t_{0}=$ const is fulfilled, and the delay $\tilde{\tau}$ can be obtained as the arrival time $t_{\mathrm{t}}$ of the calibration pulse in the trace of the calibration event ${ }^{3}$ :

$$
\tilde{\tau}=t_{\mathrm{t}}-0=t_{\mathrm{t}} .
$$

The pulse arrival time $t_{\mathrm{t}}$ is determined in a subsequent analysis as time of the positive maximum of the up-sampled trace (like shown in figure 4.4). When repeating the measurement for the same channel several times, the measured pulse arrival time $t_{\mathrm{t}}$ is stable within about one sample of the up-sampled trace (standard deviation of 12 successive events $\lesssim 0.1 \mathrm{~ns}$, trace up-sampled to $12.5 \mathrm{~ns} / 2^{7} \approx 0.1 \mathrm{~ns}$ sample spacing), if the amplitude of the calibration pulse is chosen high enough for a sufficient signalto-noise ratio. Hence, this measurement method enables us to determine the relative delays $\Delta \tau_{\mathrm{m}, \mathrm{n}}$ with a statistical error of about $\sqrt{2} \cdot 0.1 \mathrm{~ns} \approx 0.15 \mathrm{~ns}$.

Furthermore, systematic errors of the delay measurements have been studied in several ways, e.g., by repeating the measurements. Measurements of the relative

\footnotetext{
${ }^{3}$ There is a freedom of adding a constant to all $\tilde{\tau}$ without changing the relative delays. This is exploited by setting $\tilde{\tau}$ to 0 for antenna 1 , to facilitate the handling of calibration tables in the analysis software. This is an arbitrary choice which has no effect on the physics results.
} 


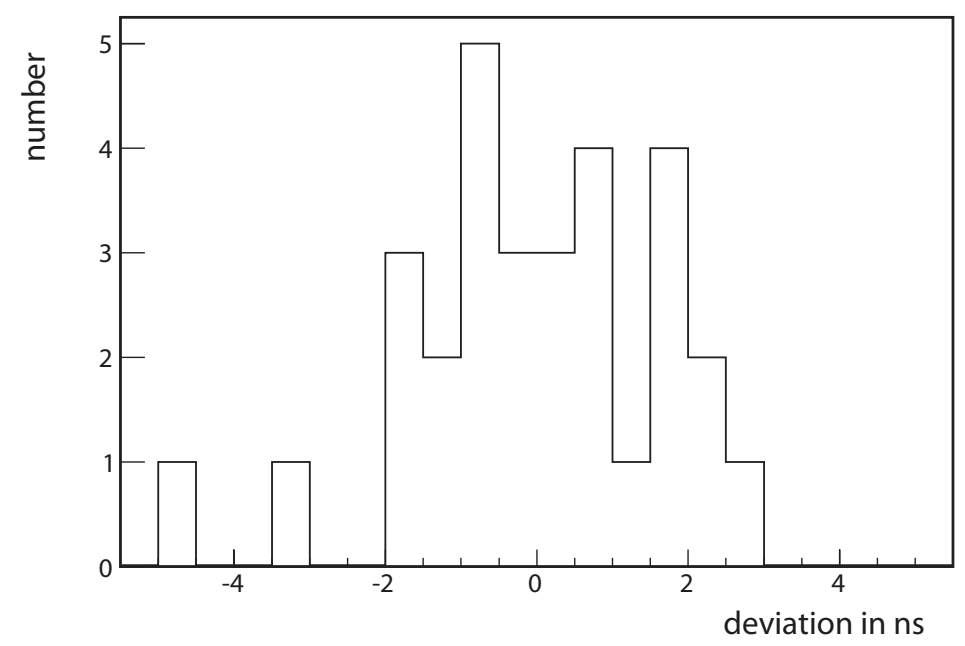

Figure 4.5.: Deviations between delays $\tilde{\tau}$ calculated by the negative extremum of the up-sampled trace and the maximum of the envelope; mean shifted to 0 , standard deviation $=1.7 \mathrm{~ns}$. The histogram contains 30 deviations of one delay measurement campaign of all 30 LOPES antennas.

delays performed on two consecutive days deviate by $(0.4 \pm 0.3)$ ns from each other (mean and standard deviation of 10 measurements). As another check for systematic effects, the pulse arrival time $t_{\mathrm{t}}$ has been determined in four different ways, namely as time of the positive maximum of the trace, the negative maximum of the trace, the maximum of a Hilbert envelope of the trace, and the crossing of half height of a Hilbert envelope of the trace (cf. appendix B). The statistical error of the relative delays is about the same for each method $(\sim 0.15 \mathrm{~ns})$. But the value of the relative delays $\Delta \tau_{\mathrm{m}, \mathrm{n}}$ depends on the way the pulse arrival times $t_{\mathrm{t}}$ are calculated. Only the relative delays calculated by the positive and negative maximum of the trace agree within the statistical error of about $0.15 \mathrm{~ns}$. The relative delays calculated by the maximum of the envelope and the crossing of half height of the envelope disagree slightly with each other, and the delays calculated by the positive or negative maximum of the trace are highly inconsistent with the delays calculated by the maximum of the envelope, as they all have a statistical error of about $0.15 \mathrm{~ns}$, but differ by up to a few nanoseconds.

Under the assumption that the electronics of all channels behaves identically, all methods for the determination of the pulse arrival times should lead to exactly the same relative delays. Hence, the explanation for the observed inconsistency is that the properties of the different channels are not exactly the same. Indeed, after correction for all measured differences, namely the amplification factor and the dispersion (see next section), the inconsistency between the delays obtained from the different methods is reduced. But still, there remains a deviation of up to a few nanoseconds for some channels, and the average deviation between the relative delays calculated by the maxima of the trace and by the envelope of the trace is of about $1.7 \mathrm{~ns}$ (see figure 4.5). This shows the difficulty to fully correct for different channel properties. Or in other words, designing the electronics for a new radio antenna array, one has to pay attention that components are from the same batches, etc. 
In the standard analysis for shower reconstruction, a cross-correlation beam is formed using the trace and not its envelope. Therefore, in the LOPES analysis the delays calculated by the time of the positive or negative maximum of the trace are used. Hence, systematic uncertainties introduced by the effect mentioned above are minimized for the cross-correlation beamforming, and thus, are only important for measurement of pulse arrival times in individual antennas. But still, another source of systematic uncertainty remains: The distance of the positive and negative maximum of the trace is about $9 \mathrm{~ns}$ because the response of the bandpass filter causes an oscillation with the center frequency of the used band. This oscillation and, thus, the distance between positive and negative maximum and the resulting relative delays depend little $(\sim 0.5 \mathrm{~ns})$ on the shape of the calibration pulse. This translates into a systematic uncertainty of the same order when determining pulse arrival times, if the pulse shape of cosmic ray radio pulses changes with lateral distance, as predicted by simulations [106].

Another check for systematic errors was done by shifting the emission time of the calibration pulse by integer and non-integer multiples of the sampling clock. No effect on the relative delays has been observed. This proves that up-sampling works reliably, and that the determination of the arrival time of radio pulses does not depend on how these pulses arrive relative to the original sampling clock. Consequently, neither upsampling nor the original sampling rate of $12.5 \mathrm{~ns}$ introduce any significant systematic errors.

Summarizing, the total error on the relative delays $\Delta \tau_{\mathrm{m}, \mathrm{n}}$ is below $1 \mathrm{~ns}$ for the standard cross-correlation beam analysis, which is sufficient for interferometric measurements with LOPES. For other analysis methods, like evaluating pulse arrival time distributions, the total error is larger due to the inconsistency of the different ways to calculate the pulse arrival time. In such a case, the uncertainty is estimated to be in the order of $2 \mathrm{~ns}$.

The relative delays obtained by the described method are consistent with those determined earlier by solar burst measurements. The new method, however, has two fundamental advantages compared to using astronomical sources: The resulting delays do not contain any systematic uncertainty related to uncertainties of the antenna positions, and the delay calibration can be done at any time. At LOPES, the delay measurement is repeated roughly once per year or when changes in the experimental setup require it. For other experiments with a higher sensitivity to astronomical radio sources, e.g., AERA or LOFAR, also a time calibration with continuously emitting astronomical sources might be possible, similarly to the solar burst method previously used for LOPES.

\subsection{Pulse distortion by dispersion}

Dispersion is the frequency dependence of the group velocity, respectively of the group delay of a system. In case of dispersion, waves at different frequencies propagate with different speeds, leading to a linear distortion of broad band radio pulses. With a network vector analyzer the phase response of the LOPES analog electronics has been measured (see figure 4.6). It shows significant dispersion which is mainly caused by the bandpass filters. Fortunately, the dispersion of the filter can be removed in the 


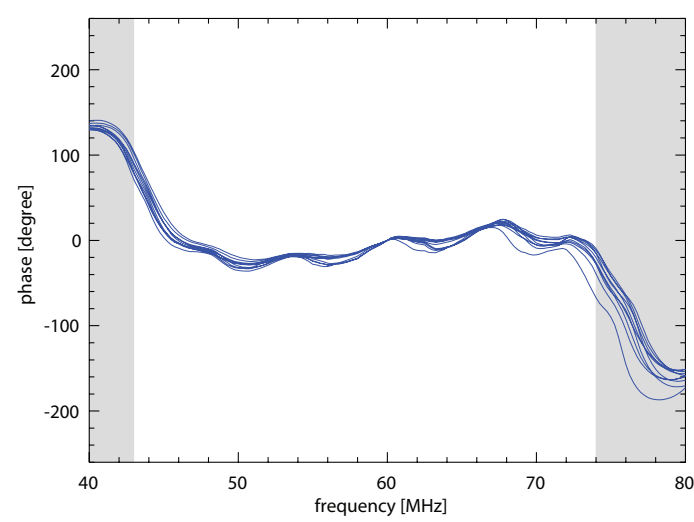

(a) phase responses of LOPES 10 electronics

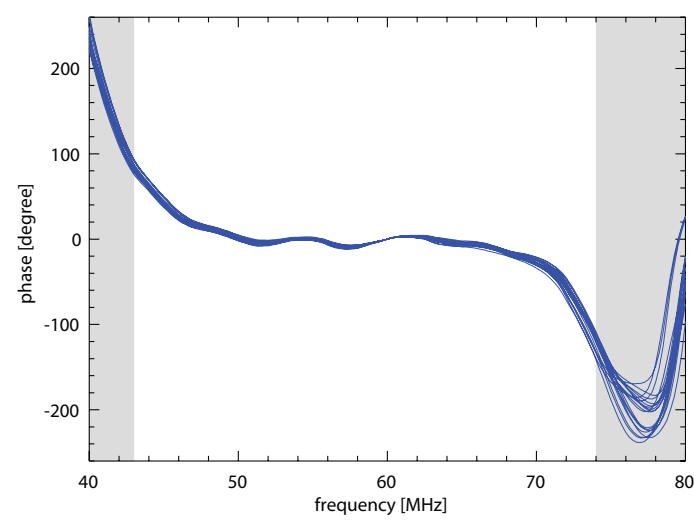

(c) phase responses of LOPES 30 electronics

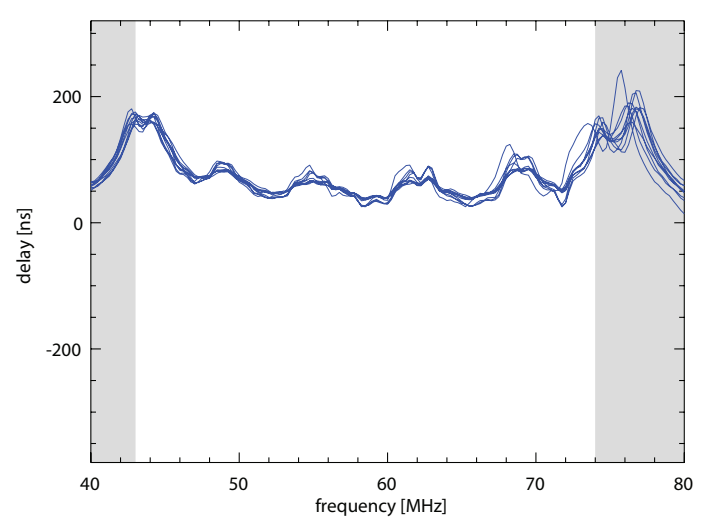

(b) group delays of LOPES 10 electronics

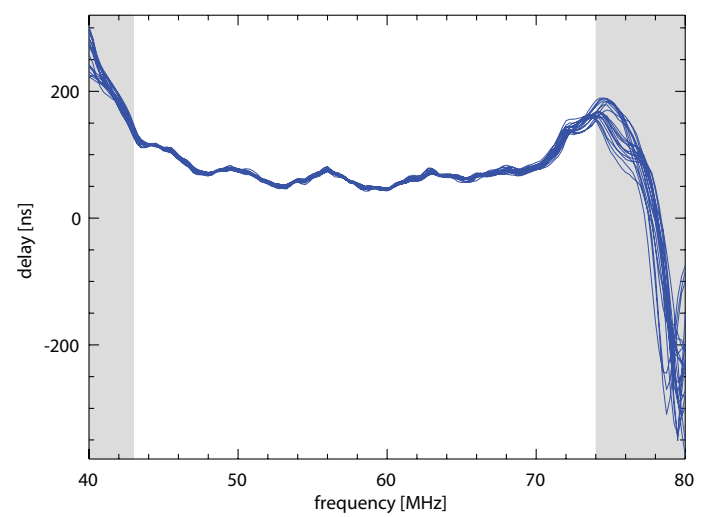

(d) group delays of LOPES 30 electronics

Figure 4.6.: Phase responses $\phi(\omega)$ of the LOPES analog electronics, and derived group delays $-\mathrm{d} \phi(\omega) / \mathrm{d} \omega$, for the original LOPES 10 electronics (above) and the electronics of the 20 additional antennas of LOPES 30 (below). The phase response has been corrected for the group delay measured at $60 \mathrm{MHz}$ by subtracting a corresponding phase gradient. Hence, deviations from $0^{\circ}$ are due to dispersion and lead to pulse distortion. The shape is dominated by the bandpass filter, but the measurement also includes amplifier and bias-tee. The gray shaded area indicates the frequency range outside of the effective bandwidth used for analysis $(43-74 \mathrm{MHz})$. The negative group delay in (d) occurs at frequencies in which the filter is already non-transmissive, an effect also known in optics [108]. 


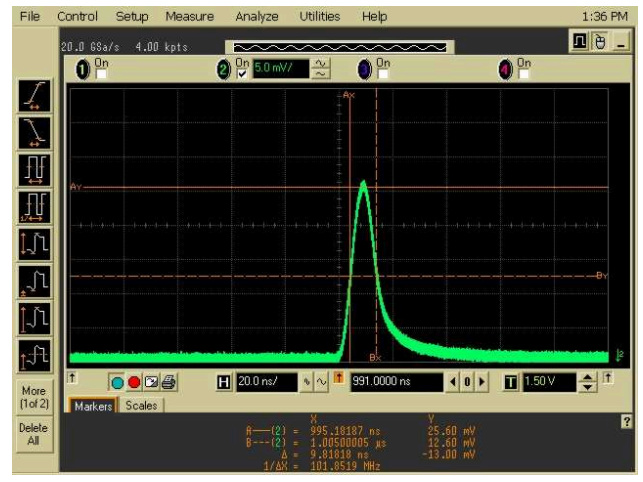

(a) narrow test pulse, input

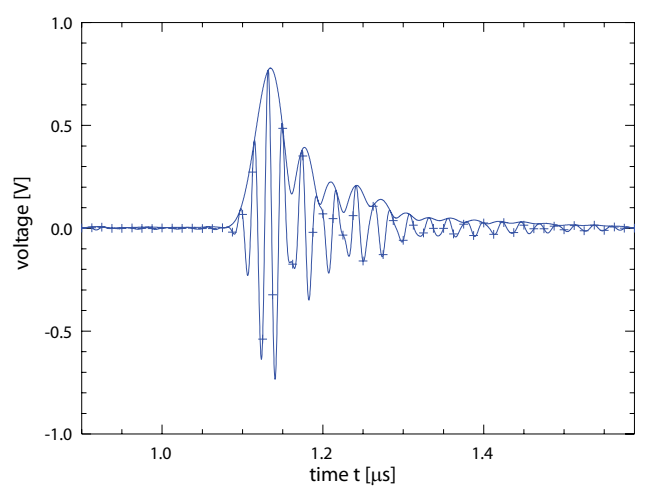

(c) narrow test pulse, raw data

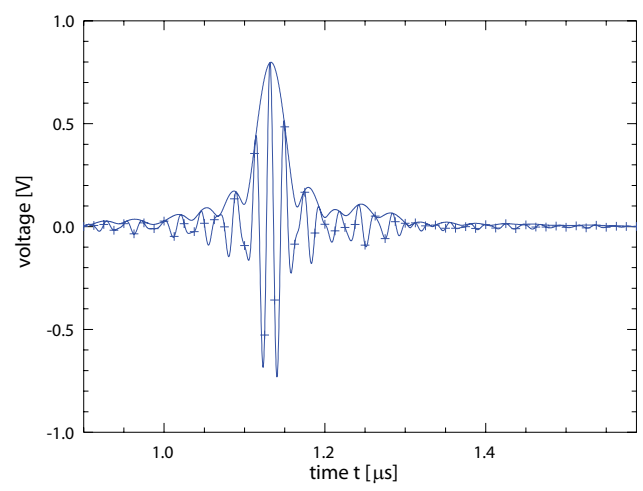

(e) narrow test pulse, dispersion corrected

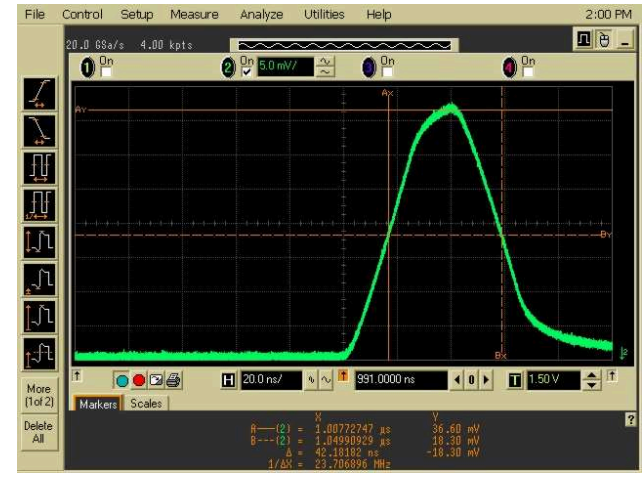

(b) broad test pulse, input

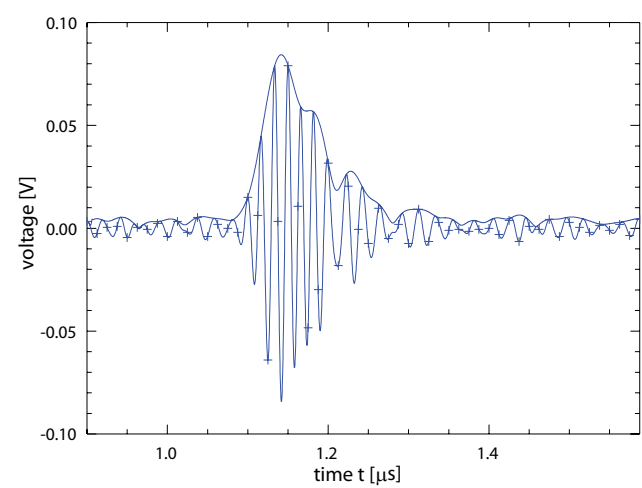

(d) broad test pulse, raw data

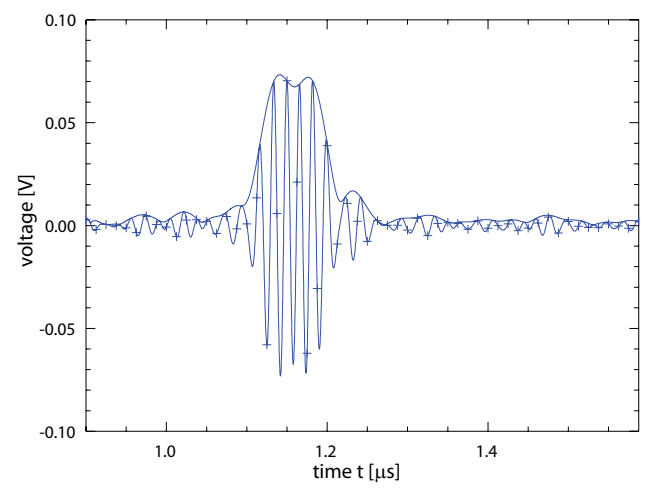

(f) broad test pulse, dispersion corrected

Figure 4.7.: Pulse distortion due to dispersion of the bandpass filter. Top: input from pulse generator. Middle: raw data of LOPES measurement. Bottom: LOPES measurement after correction for the phase response of the bandpass filter. Crosses indicate the sampled data points, and the lines correspond to the up-sampled signal and a Hilbert envelope. The dip in the middle of the broad pulse (f) arises, because LOPES is mainly sensitive to the rising and falling edges of any pulse. 
subsequent analysis by multiplying the appropriate phase corrections to the frequency spectrum.

The effect of the dispersion has been studied with test pulses from a pulse generator connected to the analog electronics instead of the antenna, like for the delay measurements. Different shapes of test pulses have been examined, and two examples are shown in figure 4.7, in which an image of the testpulse with an oscilloscope (top) can be seen, as well as the LOPES data before (middle) and after correction for the dispersion (bottom). For most pulse shapes, the dispersion leads to a change of about $10 \%$ (increase or decrease) in amplitude and width (= FWHM of a Hilbert envelope of the up-sampled field strength trace). Since the influence of the filter dispersion is largest close to the edges of the frequency band, the mentioned distortion effects can be reduced from about ten to a few percent, when using the sub-band from 43 to $74 \mathrm{MHz}$, only. For radio experiments with unknown dispersion such a selection of an inner sub-band could be a possibility to reduce systematic uncertainties originating from pulse distortions. Still, whenever possible, the dispersion should be measured and corrected during data analysis because it also affects lateral distributions (see section 7.3.2).

Because the radio pulses from real cosmic ray events are similar to the used test pulses (at least within the used frequency band), distortion effects in the same order of magnitude are expected for real events (i.e., changes of a few percent in amplitude and width). In addition, the pulse arrival time changes by up to a few nanoseconds, depending on how it is calculated (e.g., time of the pulse maximum or the crossing of half height). These are changes in the absolute value with a similar effect for all channels, because the dispersion of each channel is approximately equal (see figure 4.6), as similar electronics is used.

The impact of dispersion on the more important relative timing is little. Under the assumption that the cosmic ray radio pulse shape does not change much on the lateral extension of LOPES $(\sim 200 \mathrm{~m})$, the pulse should be distorted by every antenna and its corresponding electronics in the same way. This means that any eventually remaining difference between different antennas and their electronics is expected to be much smaller than the observed absolute shifts of a few nanoseconds. Consequently, the dispersion of LOPES, even if not totally taken into account, does not prevent the achievement of the required relative timing accuracy of about $1 \mathrm{~ns}$.

The dispersion of the LOPES antenna itself is neglected against the dispersion of the bandpass filter, which dominates the total dispersion of LOPES. The dispersion of the LOPES antenna type is not known because it is difficult to measure. The two antenna method, normally applied to measure antenna dispersion, cannot be used, since the LOPES antenna is only a receiver, but not a transmitter. Still, a qualitative evaluation of the antenna dispersion is possible: In figure 4.1, the pulse of a real cosmic ray event is corrected for the dispersion of the filters, and any remaining pulse distortion should originate from the LOPES antenna. This seems to be much smaller than the filter dispersion shown in figure 4.7. Nevertheless, due to the high noise level for real events, and because the exact shape of the cosmic ray radio pulses is unknown, this cannot be expressed quantitatively.

For LOPES ${ }^{\mathrm{STAR}}$, which uses different antennas and electronics, the dispersion of the complete system has been measured [81]. It was found that the dispersion of the cables can be neglected, but the dispersion of the antenna itself cannot. It can 


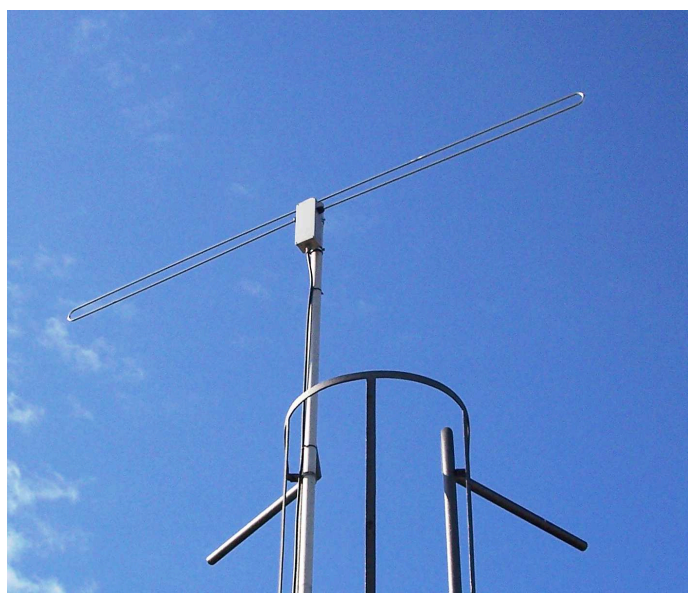

(a) dipole antenna (LOPES 30, until 2009)

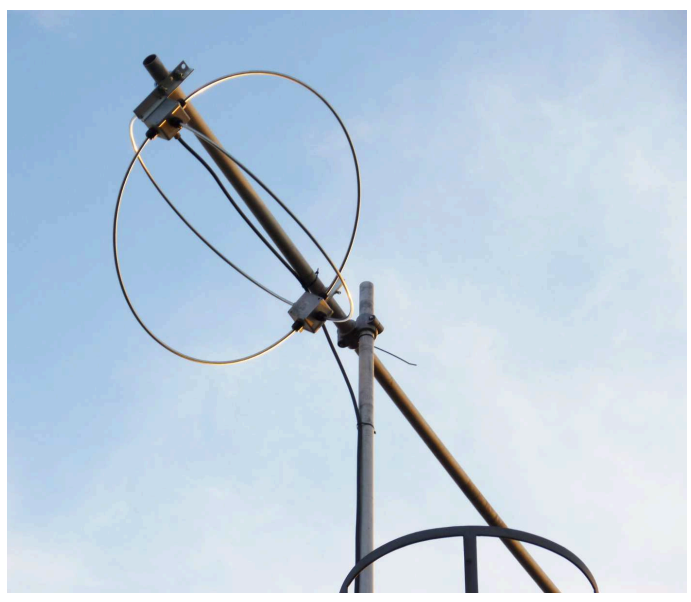

(b) SALLA (LOPES 3D, from 2010 on)

Figure 4.8.: LOPES beacon antennas.

be of the same order of magnitude as the filter dispersion. For this reason, future radio experiments should aim either for antennas with low dispersion or for antennas with well-known and, thus, correctable dispersion. For instance, for AERA, the dispersion of the antenna is not negligible, but it has been measured and will be accounted for in offline data analysis. Correcting pulse distortions by dispersion is especially important for larger-scale antenna arrays, if it turns out that the cosmic ray radio pulse shape changes with lateral distance. This could also have implications for the application of interferometric analysis methods, e.g., forming a cross-correlation beam. Hence, larger antenna arrays, like AERA at the Pierre Auger Observatory [96] or LOFAR [55], have the opportunity to investigate this.

\subsection{Monitoring of the timing with a beacon}

To continuously monitor and guarantee a nanosecond relative timing precision, a radio reference beacon is necessary, because experience with LOPES has shown that the timing is not absolutely stable. Instead, once in a while, jumps by one or two clock cycles (12.5 ns) occur. In addition, small drifts or changes in the relative delays, e.g., with changing environmental temperature, cannot be excluded, as the electronics has not been designed for sub-nanosecond stability. Independent of the reasons, any changes in the timing have to be accounted for, to achieve an overall timing accuracy in the order of $1 \mathrm{~ns}$. As the exact variations of the delay are not predictable, a continuous monitoring of the timing is needed making it possible to correct the timing in the subsequent analysis on an event-by-event basis.

For this monitoring, originally the carriers of a television transmitter had been used, but it was switched off at the end of 2007. Thus, in the beginning of 2008, an emitting horizontal dipole antenna, a beacon, was deployed in the Karlsruhe Institute of Technology, at about $400 \mathrm{~m}$ distance to the center of LOPES (figures 4.8 and 3.1 ). The beacon permanently transmits two sine waves at constant frequencies of $63.5 \mathrm{MHz}$ and $68.1 \mathrm{MHz}$ (width $<100 \mathrm{~Hz}$ ) at a low power of $-21 \mathrm{dBm}(\approx 0.008 \mathrm{~mW})$. 
Thus, every LOPES event contains a measurement of the phasing at these frequencies, which can be obtained by a Fourier transform into the frequency domain. Any variation in the relative timing between two antennas can thus be detected as a variation of the phase differences at each beacon frequency.

Also other experiments use beacons for time calibration, but in a different way. For example, ANTARES [109], ANITA [110] and AURA [111] determine the arrival times of pulses emitted by a beacon. In addition, AURA has the capability to measure frequency shifts of constant waves for calibration [68]. Evaluating phase differences of a continuously emitting radio beacon is reported for ionospheric TEC measurements [112], i.e. for atmospheric monitoring, but not for time calibration. The following paragraphs explain the beacon method used at LOPES, and in future at AERA (see chapter 5), which is essentially based on phase measurements of stable, monofrequent beacon signals.

Beacon method The phase of the continuous beacon signal at an antenna depends on the distance and the orientation angle of the antenna towards the beacon as well as on the delay of the corresponding channel. Let us assume for a moment that there are two antennas at an equal distance and angle to the beacon and with an equal delay. If we consider just one beacon frequency, e.g. $63.5 \mathrm{MHz}$, the two antennas would measure the same phase at this frequency (within a small uncertainty due to noise). Thus, a variation in the relative delay between the two antennas would immediately result in a change in the measured phases. If, e.g., the relative delay shifts by $1 \mathrm{ns,}$ the difference between the measured phases at the two antennas would be $\Delta \phi=1 \mathrm{~ns}$ $\cdot 63.5 \mathrm{MHz} \cdot 360^{\circ}=22.9^{\circ}$. Correspondingly, a measured phase difference can be converted into a shift of the relative delay.

Now let us consider the more realistic case that we have two antennas with different angle and distance towards the beacon and different electronics and cable delays. As the distance and the effect of the antenna orientation is not precisely known (because there is no need), we expect to measure a different phase at both antennas at the beacon frequency. As long as neither the distance, nor the orientation, nor the relative delay do change, the difference between the phases measured at both antennas $\Delta \phi_{\text {ref }}$ would be arbitrary, but constant. Thus again, changes in the relative delay can be detected as changes in the phase difference $\Delta \phi$. The only difference to the case above is that these changes in the phase differences will happen not with respect to $0^{\circ}$, but with respect to $\Delta \phi_{\text {ref }}$.

The important point is to define $\Delta \phi_{\text {ref }}$ for each beacon frequency, for each antenna with respect to a fixed reference antenna (arbitrary choice) when the delay is exactly known. Therefore, $\Delta \phi_{\text {ref }}$ is determined as the average phase difference of the events taken during a delay calibration campaign (cf. section 4.4). This way, any timing variations can be monitored and subsequently corrected back to the values obtained in the delay calibration.

Uncertainties and systematic effects The precision of the measurement of phase differences is limited by noise and systematic effects. The uncertainty due to noise depends on the signal-to-noise ratio of the beacon signal (within reasonable limits, see chapter 5), whereby the amplitude of the beacon emission can be chosen such that 


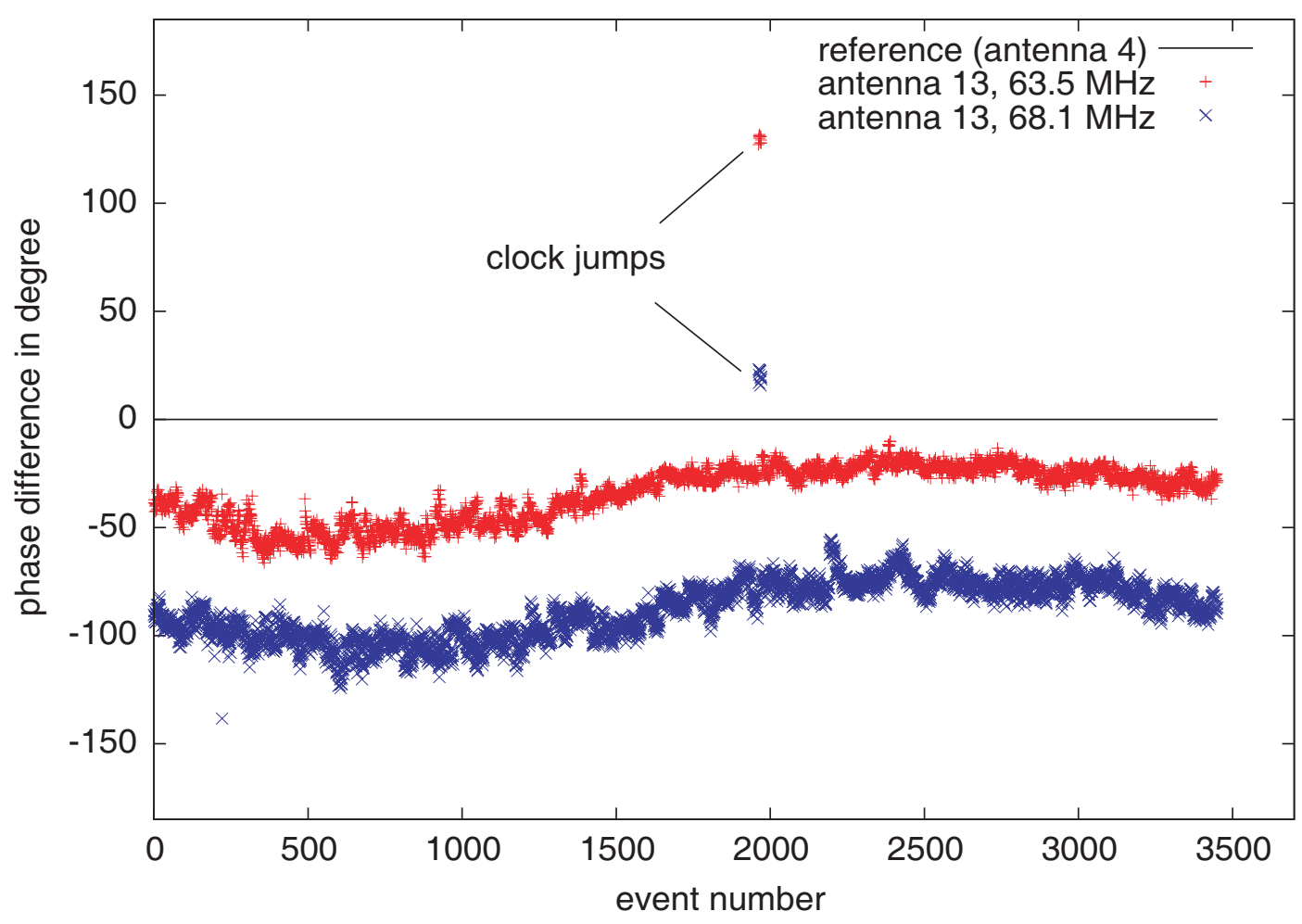

Figure 4.9.: Phase differences between two antennas at both beacon frequencies for the first ten events per day for an entier year (May 2008 - May 2009), excluding a few days of down time. The absolute value of the phase differences is not meaningful. However, the changes are, which amount at both frequencies to about $1.5 \mathrm{~ns}\left(\sim 35^{\circ}\right)$ between summer and winter. Details see text.

a sufficient precision is achieved. For LOPES 30, an emission power of $-21 \mathrm{dBm}$ was chosen for each frequency, which was increased to about $-10 \mathrm{dBm}$ for the LOPES 3D setup. The uncertainty due to noise of the phase measurement has been determined as the jitter of the phase differences in successive events. It corresponds to an relative timing uncertainty of $\sim 0.3 \mathrm{~ns}$.

Aside from that, one might be cautious that the beacon introduces RFI and disturbs the measurement of radio pulses. However, this disturbance is negligible, as the cosmic ray radio pulses are broad band and extend over the entire frequency spectrum. On the other hand, the beacon signal is visible only in a few fixed and defined frequency bins and can be suppressed by artificially reducing the amplitude at these bins in the data analysis, or in the hardware of the trigger logic if a radio self-trigger system is applied.

In figure 4.9, the phase differences at both beacon frequencies between two LOPES antennas are shown for the first ten events of each day of one year. An annual drift in the phase differences which corresponds to about $1.5 \mathrm{~ns}\left(\sim 35^{\circ}\right)$ can be seen consistently at both frequencies. The reason for this annual drift is not totally understood, yet, but might be due to environmental effects, in particular changing 
temperature, as the effect is largest in summer and winter. Also a jump in the timing of two clock cycles $(25 \mathrm{~ns}$ ) is visible which occurs during one day. Here it becomes obvious that at least two beacon frequencies are needed, as changes in the timing larger than half a period ( $\sim 9 \mathrm{~ns})$ could otherwise not be detected unambiguously. A consistency check between the results at both frequencies is also necessary to identify a few noisy events (like the outlier in the bottom left corner of figure 4.9), for which the beacon correction of the timing cannot be performed.

When inspecting the data carefully, some features in the plot of the phase differences can be seen which do not occur simultaneously at both frequencies, contrary to the visible general drift. These features are due to systematic effects and in principle decrease the achievable timing precision. Possible reasons for systematic effects are changes in the emitted beacon signal (e.g., if the frequency generation is not absolutely stable), changes in the propagation of the signal from the beacon to the LOPES antennas (e.g., due to different atmospheric or ground properties), and nonrandom (e.g., human-made) RFI at the beacon frequencies. Since the scale of the observed features is significantly smaller than $1 \mathrm{~ns}$, they have not been investigated in detail, and should not limit the ability of the beacon method to provide a timing precision of $1 \mathrm{~ns}$.

As a cross-check, the changes in the relative timing between two dates roughly one year apart have been measured with the method described in section 4.4 (delay measurement), and compared to the changes in the beacon phase differences between the same two dates (beacon measurement). The delay measurement yielded a change by $(0.6 \pm 0.4)$ ns between the two dates (mean and standard deviation of the absolute change of all 30 antennas). This itself is not unexpected as the electronics was not designed to be stable on a sub-nanosecond level. However, the comparison with results of the beacon measurement reveals some systematic effects. In the ideal case, the measurement at both beacon frequencies should exactly correspond to the delay measurement. In reality, the results obtained at the two different beacon frequencies are not totally equal, but differ by $(0.6 \pm 0.3) \mathrm{ns}$. This is larger than the statistical error which is about $0.3 \mathrm{~ns}$ (see above). Moreover, the average result of both beacon frequencies deviates by $(0.7 \pm 0.5) \mathrm{ns}$ from the delay measurement. This indicates, first, that the measurement at both beacon frequencies is inconsistent with each other, and second, that the beacon measurement is inconsistent with the delay measurement. However, the significance is not very high, and the absolute amount of both inconsistencies is smaller than the required relative timing precision of $\sim 1 \mathrm{~ns}$.

Nevertheless, systematic effects on the beacon signal seem to be important. It cannot be excluded that the drifts observed in the phase differences are - at least partly - due to these systematic effects, and not due to real drifts in the electronics or cable delays. Nevertheless, this does not undermine the ability of the beacon method to monitor and correct real changes in the timing, like the described clock jumps, or sub-nanosecond time shifts due to noise in the digital electronics. Thus, the beacon can provide for each event a timing precision in the order of $1 \mathrm{~ns}$, which is required for digital radio interferometry.

Outlook Beside its use to monitor the timing of an antenna array, a beacon is also valuable to check the health of the experimental setup in general. As the beacon 
provides a defined reference signal visible in each event, it is feasible to detect almost any failures of the antennas or the electronics. For example, by investigating the phase differences at the beacon frequencies it was possible to exactly determine the date when the cables of the two polarization channels of one LOPES antenna were accidentally switched. Moreover, the beacon has been included in the online monitoring system of LOPES 3D [86]. For AERA, both features of the beacon will be exploited, i.e., improvement of the timing precision, and monitoring (see next chapter).

\subsection{Conclusions on the time calibration of LOPES}

The methods described for the time calibration of LOPES are especially useful for radio antenna arrays in a noisy environment, where the calibration with astronomical sources is not possible. They allow the determination of the electronics and cable delays with a very high precision, which can in principle be below 0.5 ns. Systematic effects, however, limit the actual achieved precision of the delay measurement to below $1 \mathrm{~ns}$ for the standard, interferometric cross-correlation beam analysis, and to about $2 \mathrm{~ns}$ for the direct measurement of pulse arrival times at individual antennas. In addition, the dispersion of the electronics has been measured and is taken into account in the analysis of air shower induced radio pulses, to avoid systematic uncertainties in the pulse amplitude of $\lesssim 10 \%$.

Furthermore, variation of the timing are continuously monitored with narrow band reference signals from a beacon, providing an overall timing precision in the order of $1 \mathrm{~ns}$ for the cross-correlation beam analysis (see table 4.3). This way, the nanosecond timing precision is achieved for each event, and the phased antenna array LOPES can be used as a digital radio interferometer.

Finally, monitoring of the timing with a beacon is an interesting feature for any radio antenna array. As in principle the phasing at the beacon frequencies is sensitive to any variations of the relative timing, even the relative timing precision of antenna arrays without stable clocks can be improved to about $1 \mathrm{~ns}$. Hence, a beacon should provide any radio array in the $\mathrm{MHz}$ regime with the capability to do interferometric measurements. For example, the application of a beacon is investigated at AERA, and results of this investigations are presented in the next chapter. However, despite the remarkable precision of the time calibration, one must not forget that noise is the source of an additional uncertainty, which can exceed the calibration uncertainty (cf., chapter 6). 
Table 4.3.: Summary of uncertainties for the relative timing, and the relevance for the interferometric cross-correlation beam (CCbeam) analysis

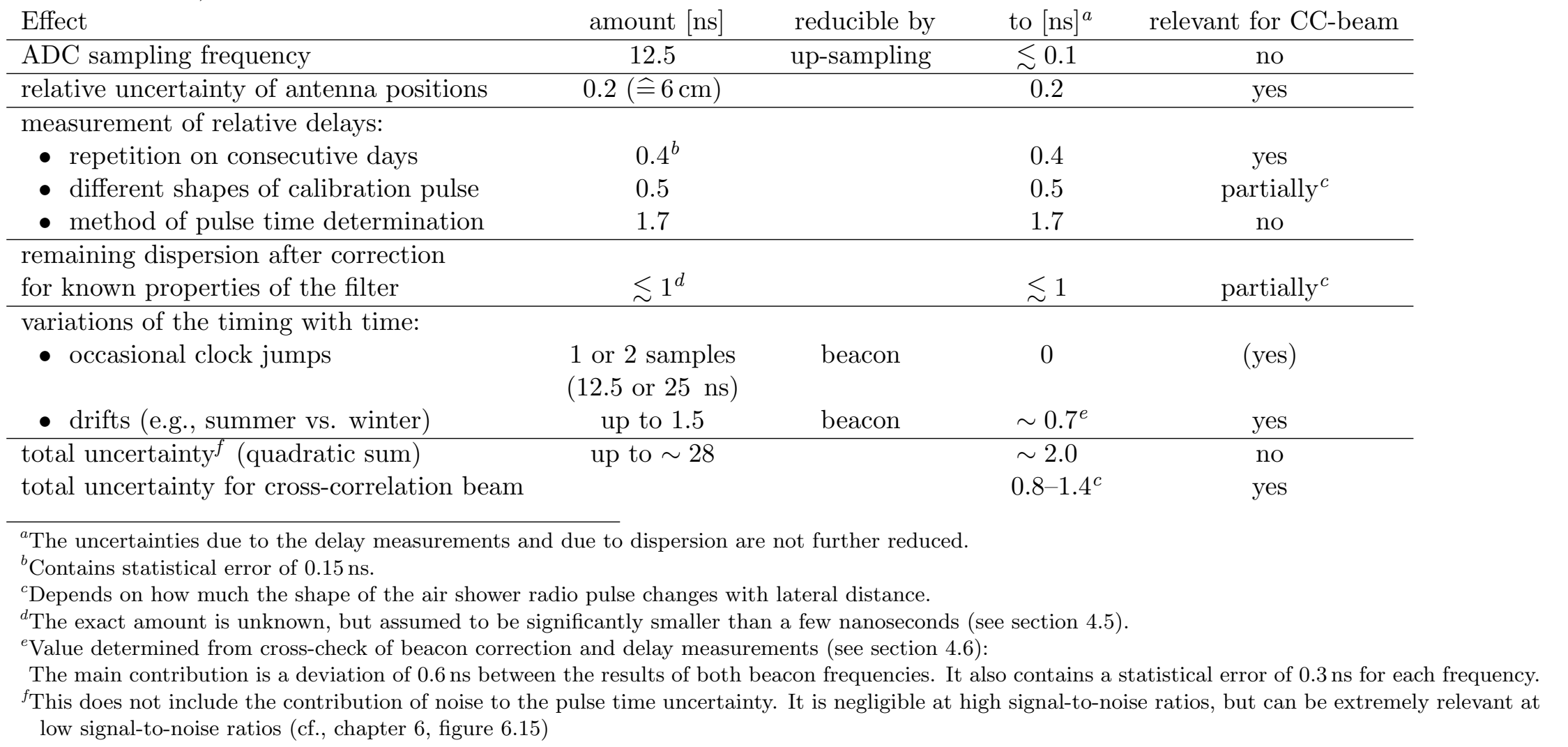




\section{A Reference Beacon for the Auger Engineering Radio Array}

Using the Auger Engineering Radio Array (AERA) as a digital radio interferometer requires about the same relative timing precision and accuracy of $\sim 1 \mathrm{~ns}$ as for LOPES, because AERA operates in a similar frequency range $(30-80 \mathrm{MHz})$ as LOPES $(40-80 \mathrm{MHz})$. In contrast to LOPES which is externally triggered, AERA is planned to record self-triggered events with a high signal-to-noise ratio sufficient to detect the radio pulse already in individual antennas. An event whose signal-tonoise ratio is so low that it could only be detected with interferometry will not be triggered. Thus, interferometry will not lower the detection threshold of AERA.

Nevertheless, AERA can benefit from the improved signal-to-noise ratio and precision of an interferometric cross-correlation beam analysis. The signal-to-noise ratio of the cross-correlation beam scales with the number of antennas, while the precision of any fit to the lateral distribution is supposed improve with the square root of the number of antennas, only [42]. In addition, beamforming allows to exploit also those antennas for reconstruction which have not triggered themselves because of a weak signal, but were triggered by other antenna stations. Furthermore, the angular resolution of an interferometric reconstruction ought to be better than the one of a simple arrival time triangulation, because interferometry is not only sensitive to the arrival time of the radio pulse but also to the oscillation of its electric field.

However, it has not been studied so far, how much the reconstruction of arrival direction, energy and possibly composition can be improved by applying interferometric methods. In addition, it is not clear, up to which lateral distances interferometric methods are useful. There presumably exists a limit, since cross-correlation beamforming assumes that all antennas receive exactly the same radio pulse (just at a different time), but simulations predict the pulse shape to change with lateral distance (cf., figure 2.4). Investigating the prospects of interferometry at large scales is one of the major goals of AERA.

This chapter describes how the relative timing precision required for interferometry is achieved at AERA using a beacon. At AERA, the beacon has not only the duty to monitor the relative timing, like at LOPES, but also to improve the timing precision provided by GPS from a few ns to $\lesssim 1 \mathrm{~ns}$. The total relative timing precision (and accuracy) depends also on the analog and digital electronics of the AERA stations, and has to be checked with separate calibration measurements. However, such calibration measurements are not covered by this thesis, because the construction of AERA phase 1 was delayed. Nevertheless, several test measurements have been performed to demonstrate that a beacon can indeed improve the relative timing precision. Afterwards, a beacon system meeting the special requirements of AERA, has been developed, installed and tested. 

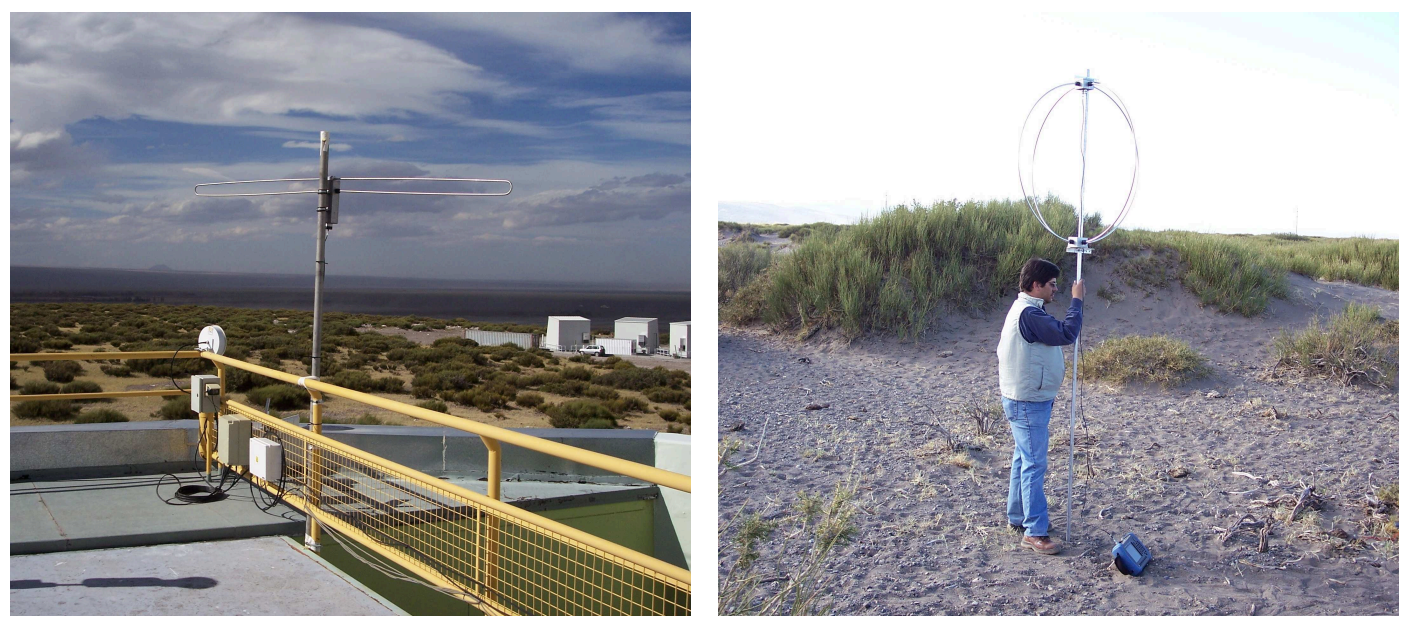

Figure 5.1.: Left: preliminary test beacon at the roof of Coihueco; right: measurement of the beacon power at the AERA site with a mobile, $1 \mathrm{~m}$ diameter SALLA on a $3 \mathrm{~m}$ pole and a spectrum analyzer.

\subsection{Proof of principle}

As already discussed in section 4.6, changes in the relative timing between two antenna stations can be detected by measuring phases of a mono-frequent signal emitted by a reference beacon. The phase is obtained from the complex frequency spectrum calculated by a Fourier transform of the electric field strength trace. The phase difference $\Delta \phi$ between different antennas is the difference of the phase measured at one antenna station and the phase measured at another antenna station. $\Delta \phi$ should be constant if the relative timing of the stations is constant, and variations of the relative timing can be derived from variations of $\Delta \phi$.

For LOPES, this method has been used to detect variations of the relative delays over days and months by monitoring changes in the mean of $\Delta \phi$. For AERA, the relative timing between different stations varies on an event-by-event basis, as no common clock is distributed via cables, but each station has its own GPS clock. The time stamps obtained by GPS at different stations have a precision relative to each other of about $2.3 \mathrm{~ns}$, for the MAXIMA prototype stations at the BLS [113], as well as for the AERA stations of phase 1. However, the AERA stations are planned to be upgraded with improved digital electronics, whose design specifications allow a relative timing precision of $\lesssim 5 \mathrm{~ns}$ - determined by the internal clock rate of $180 \mathrm{MHz}$ and the used GPS module (cf. also reference [114]). This means, the GPS time stamp has to be improved by a factor of $\sim 5$ to achieve a relative timing precision of about $1 \mathrm{~ns}$ required for digital interferometry.

The feasibility of the beacon method has been tested in two steps. First, a preliminary beacon was installed at the Coihueco telescope building to measure how well the beacon signal could be received at different locations at the AERA site. Second, the preliminary beacon was installed at the BLS to determine how much the relative timing precision between the MAXIMA prototype stations can be improved, and how this improvement scales with the signal-to-noise ratio of the beacon signal. ${ }^{1}$

\footnotetext{
${ }^{1}$ Details about the beacon test measurements can be found in GAP note 2009-085 [115].
} 


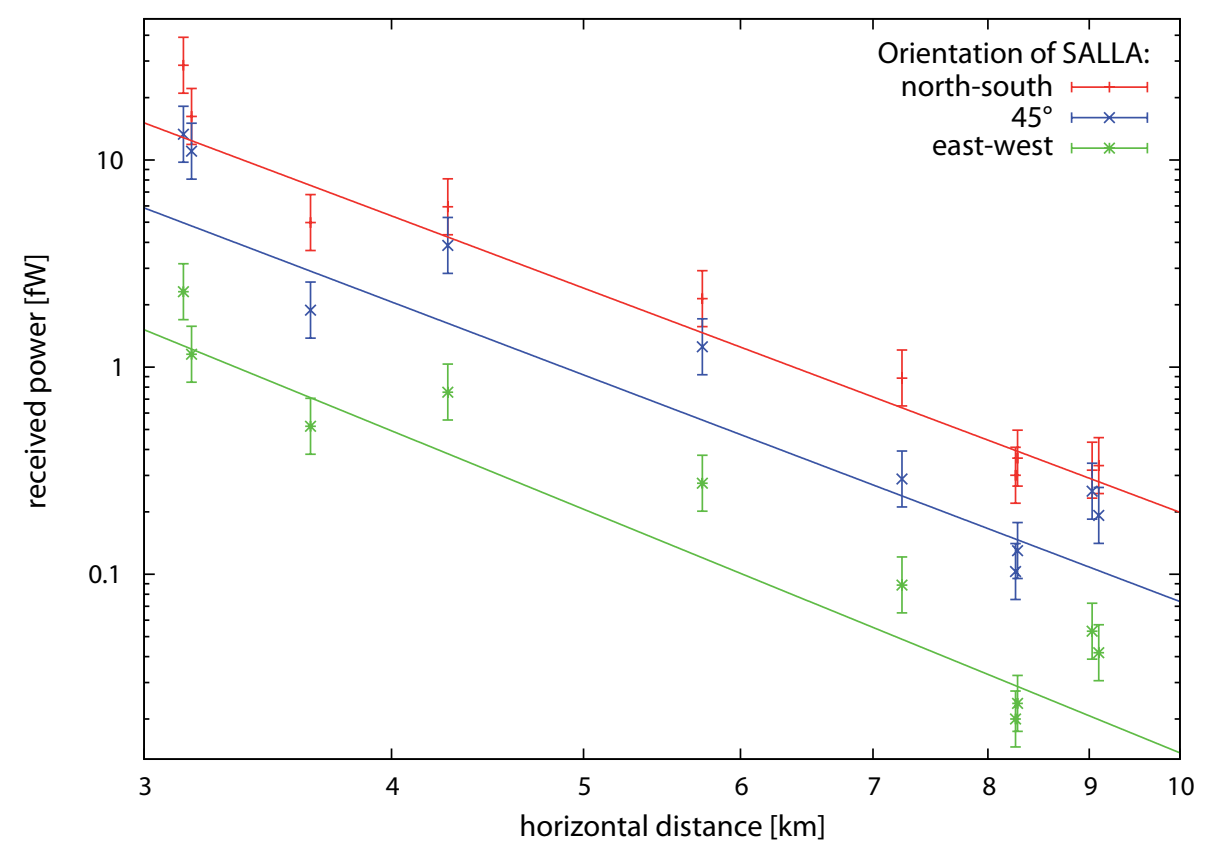

Figure 5.2.: Received power of the $67.2 \mathrm{MHz}$ beacon signal emitted from the roof of Coihueco, measured with a mobile SALLA and a spectrum analyzer in the AERA site, and power law fits to the data.

\subsubsection{Test measurements at the AERA site}

To test the reception quality of the beacon reference signal at the AERA site, a preliminary beacon system was installed at the roof of Coihueco (figure 5.1, left). It consisted of a dipole antenna connected to a frequency generator. Subsequently sine waves at different frequencies were emitted with a power of about $10 \mathrm{dBm}$ (= $10 \mathrm{~mW}$ ). The received power was measured at several positions at the AERA site with a transportable $1 \mathrm{~m}$ SALLA connected to a spectrum analyzer (figure 5.1, right). The measurement positions had been chosen to cover almost the whole extension of the AERA site. The ground distances between the measurement positions and the beacon were determined with GPS.

This way, the decrease of the received beacon amplitude with distance to the beacon was determined under realistic conditions. The results for one of the tested beacon frequencies is shown in figure 5.2. For each measurement position, the measurement was repeated with different orientations of the receiving SALLA antenna. As expected, the received signal power is maximum when the SALLA is aligned parallel to the emitting beacon antenna (north-south), and minimum for the orthogonal east-west orientation: $(10.0 \pm 2.4) \mathrm{dB}$ less than the maximum.

For each frequency and each orientation, a power law has been fitted to the received power $P$ as function of the horizontal distance $r$ to the beacon (see table 5.1). The power decreases significantly faster than with $r^{-2}$ which would be expected for free space propagation. In fact, the obtained power law indices range from -3.2 to -4.7 which is also consistent with the values obtained with the final beacon system [116]. This is not surprising, since the free space approximation is only valid, if the first 
Table 5.1.: Resulting exponents $q$ of power law fits $P(r)=$ const $\cdot r^{q}$ to the received power $P$ of the beacon signal at a distance $r$ from the beacon.

\begin{tabular}{lcc}
\hline SALLA orientation & frequency $[\mathrm{MHz}]$ & exponent q \\
\hline north-south & 49.61 & $-3.36 \pm 0.22$ \\
north-south & 56.25 & $-3.78 \pm 0.36$ \\
north-south & 67.19 & $-3.60 \pm 0.29$ \\
\hline $45^{\circ}$ & 49.61 & $-3.20 \pm 0.36$ \\
$45^{\circ}$ & 56.25 & $-3.39 \pm 0.72$ \\
$45^{\circ}$ & 67.19 & $-3.63 \pm 0.51$ \\
\hline east-west & 49.61 & $-4.70 \pm 0.66$ \\
east-west & 56.25 & $-4.08 \pm 0.60$ \\
east-west & 67.19 & $-3.91 \pm 0.42$ \\
\hline
\end{tabular}

Fresnel zone is free of any obstacles. This is the case, if the direct path from the emitter to the receiver, $l_{\text {direct }}$, is at least half a wavelength $\lambda$ shorter than the path including one reflection at any obstacle (e.g., the ground), $l_{\text {with reflection }}$ :

$$
\text { First Fresnel zone free, if } \quad l_{\text {with reflection }}>l_{\text {direct }}+\lambda / 2
$$

Even for the closest point of the AREA site at about $3 \mathrm{~km}$ distance from Coihueco and the upper band limit of $80 \mathrm{MHz}$, the ground is inside the first Fresnel zone. Consequently, the emitted beacon power is partially absorbed by the ground and decreases faster than in free space. This cannot be avoided for AERA, since it would require unrealistic antenna heights of more than $100 \mathrm{~m}$.

The decrease of the signal with distance had to be taken into account for the design of the final beacon system. As is shown in the next section, a minimum signal-to-noise ratio of the beacon signal is required to improve the relative timing precision sufficiently. Consequently, the beacon power must be high enough to achieve this signal-to-noise ratio even at the most distant antennas. At the same time, the beacon signal must not saturate the closest antennas. This means for AERA that the difference of the beacon power between the closest and most distant antenna must not exceed $\sim 20 \mathrm{~dB}$. This requires a certain minimum distance between the beacon and AERA, which is achieved with a beacon at Coihueco for AERA phase 1 , and probably also phase 2 , but most likely not anymore for the final extension of AERA in phase 3. A possible solution could be a dedicated beacon station at a larger distance to AERA of $\gtrsim 10 \mathrm{~km}$ with either an increased emission power or a more directive beacon antenna.

\subsubsection{Test measurements at the BLS}

To demonstrate that the beacon method indeed improves the relative timing precision when each antenna station has its own GPS clock, the preliminary beacon was installed at the BLS close to the MAXIMA prototype setup. The relative timing precision between two MAXIMA stations (3 and 4) was determined before and after the correction with the beacon signal, for events recorded coincidently at both stations with a forced $0.1 \mathrm{~Hz}$ trigger. Moreover, the dependency of the timing precision 


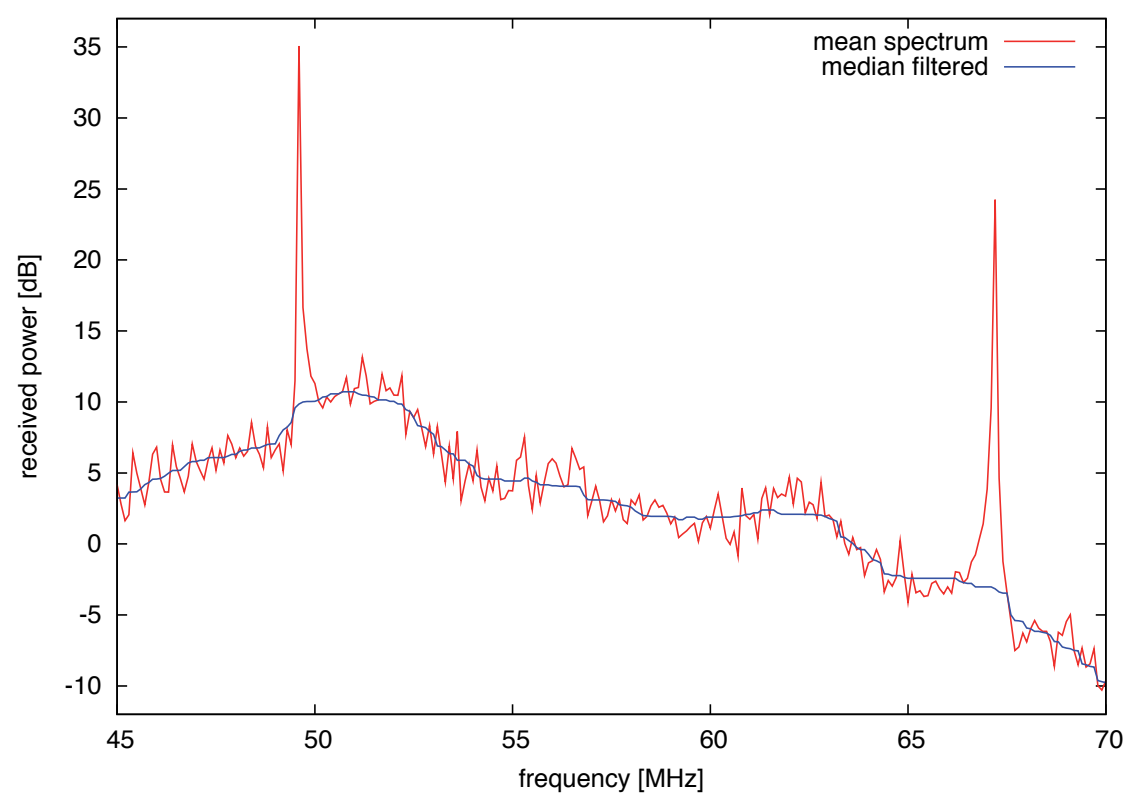

Figure 5.3.: Spectrum measured with MAXIMA station 4, north-south polarization, before (red) and after the application of a median filter (blue) which suppresses the narrow band lines of the beacon.

on the beacon power was tested with the SALLA prototype setup at the BLS.

For the test with the MAXIMA stations, the preliminary beacon emitted two frequencies $(49.6 \mathrm{MHz}$ and $67.2 \mathrm{MHz})$. The emission power could be tuned over a range of $24 \mathrm{~dB}$ (emission amplitude from 0.625 to $10 \mathrm{Vpp}, \mathrm{Vpp}=$ Volts from peak to peak). Figure 5.3 shows a spectrum of the beacon measurements recorded with an emission amplitude of $2.5 \mathrm{Vpp}$. Obviously, the beacon signal can be suppressed by a median filter to prevent a disturbance of normal data analysis. The analysis of the beacon data has been performed with the Offline software package (cf., section 3.3.3). For this, the amplitude and phase at each beacon frequency were determined by a Fourier transform, the same way it is done for the LOPES beacon (cf., section 4.6).

Phase differences at the beacon frequencies were determined per event for different data sets of typically a few 100 events recorded during a few hours. There are eight possible combinations for phase differences between the two MAXIMA stations (2 beacon frequencies $\times 2$ polarizations $\times 2$ involved stations). For each event, all eight phase differences can be converted to time differences by assuming that a full cycle of $360^{\circ}$ corresponds to a full period of the used beacon frequency. Since the absolute value of the phase differences is not physically meaningful, but only variations are, the mean phase difference is subtracted from each phase difference. This way, the time differences scatter around $0 \mathrm{~ns}$ (see figure 5.4 for an example). These time differences are a measure for time deviations per event of the MAXIMA stations relative to each other. The average absolute deviation is about $2.5 \mathrm{~ns}$ which is in agreement with other measurements of the MAXIMA GPS timing precision [113]. 


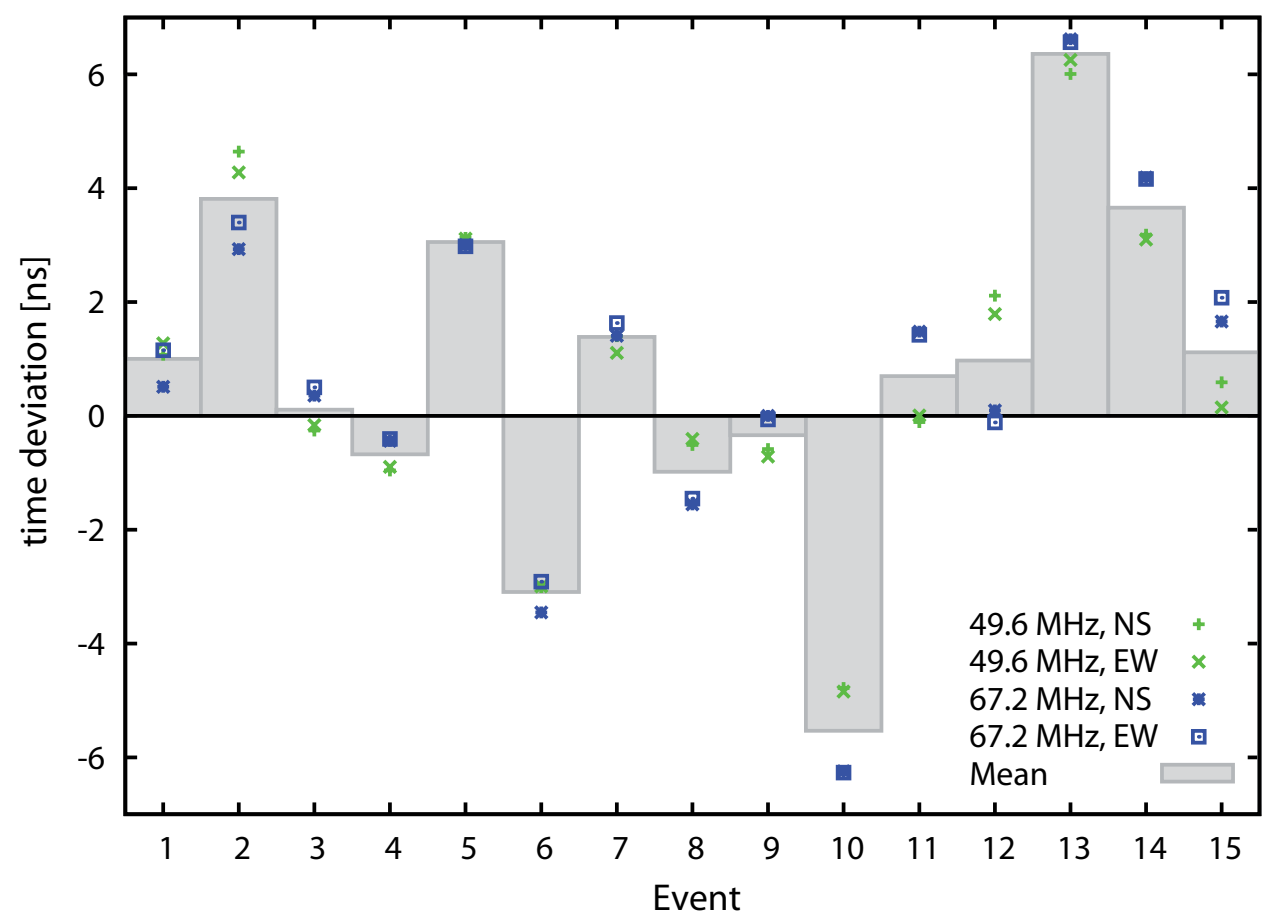

Figure 5.4.: Relative time deviation between MAXIMA stations 3 and 4, measured with the beacon (before improvement of the relative timing). For illustration, only a few successive events are shown. For each event, the time deviation is measured at both beacon frequencies, between the northsouth polarization (NS) of MAXIMA 3 and both polarizations (NS + EW) of MAXIMA 4.

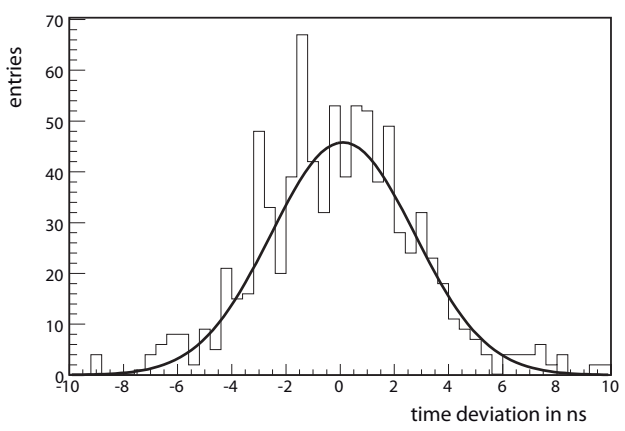

(a) before correction

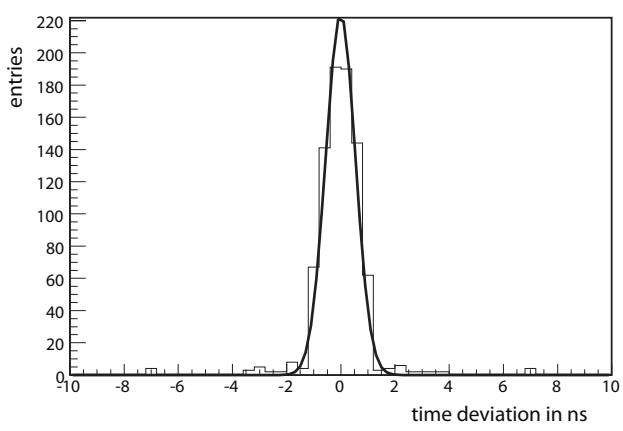

(b) after correction

Figure 5.5.: Time deviations between the MAXIMA stations 3 and 4, for about 100 events at a beacon amplitude of $10 \mathrm{Vpp}$ ( 8 entries per event, see text). Gaussian fits yield standard deviations of $2.64 \mathrm{~ns} \pm 0.09 \mathrm{~ns}$ before correction, and $0.54 \mathrm{~ns} \pm 0.01 \mathrm{~ns}$ after correction of the relative timing with the beacon signal. 
Table 5.2.: Standard deviations (RMS) of the time deviations between MAXIMA stations 3 and 4, obtained by a Gaussian fit, before correction (raw) and after correction of the relative timing with the beacon. The RMS is only an upper limit to the timing precision before correction, since the measurement quality depends on the beacon amplitude (not reflected by the statistical errors).

\begin{tabular}{lcc}
\hline beacon amplitude [Vpp] & RMS, raw [ns] & RMS, corrected [ns] \\
\hline 10 & $2.64 \pm 0.09$ & $0.54 \pm 0.01$ \\
5 & $2.95 \pm 0.22$ & $0.45 \pm 0.02$ \\
2.5 & $2.79 \pm 0.08$ & $0.67 \pm 0.02$ \\
1.25 & $3.82 \pm 0.07$ & $2.11 \pm 0.05$ \\
0.625 & $4.41 \pm 0.08$ & $3.02 \pm 0.06$ \\
\hline
\end{tabular}

Once the deviation of the relative timing from its mean value has been measured for each event, it can be corrected during data analysis, e.g., by multiplying an appropriate phase gradient to the frequency spectrum. In this way, the relative timing precision originally dominated by the GPS clocks, is significantly improved. Figure 5.5 shows two histograms of the measured time deviations between the two MAXIMA stations, before and after correction with the beacon signal. Since the precision of the phase measurements depends on the signal-to-noise ratio, the measured distributions of time deviations provide only an upper limit to the real relative timing precision before improvement by the beacon. Furthermore, the relative timing precision after improvement decreases, once the beacon power drops below a certain threshold (see table 5.2). This threshold is at a beacon amplitude of $\lesssim 2.5 \mathrm{Vpp}$ which corresponds to a $20 \mathrm{~dB}$ signal-to-noise ratio of the received beacon power. At higher beacon power, i.e., at a sufficient signal-to-noise ratio, the relative timing precision after improvement is almost a factor 2 better than required for digital radio interferometry. This provides a safety margin, if the relative timing precision of AERA should decrease by any systematic uncertainties on larger time scales, e.g., due to environmental effects.

The noise dependent uncertainty of phase measurements at the beacon frequencies has been investigated in more detail to estimate the beacon power required for AERA. The SALLA setup at the BLS has been used. Since all SALLAs are sampled using a common GPS clock, they should have an almost constant relative timing, and phase differences between different stations should be constant. Thus, they are ideal to investigate the effect of noise and systematic uncertainties on the measurement of the beacon signal. Therefore, the beacon emission power was varied over a range of $40 \mathrm{~dB}$, and at each power, the measurement uncertainty was evaluated as standard deviation of the phase differences.

The measurement uncertainty decreases with increasing beacon power, but not exactly proportional to the square root of the power, as expected from statistical uncertainties. Instead, the decreasing uncertainty can be described by a power law with an index of $-0.41 \pm 0.01$, determined with an average fit of many measurements. Individual measurements disagree within their statistical error - another sign for the presence of systematic effects. These systematic effects are probably specific to the 
used system and limit the predictability for other systems. Still, the general behavior that the precision of the phase measurement increases with increasing beacon power has been proven (for more details see [115]). Consequently, a nanosecond relative timing precision will only be achievable for AERA, if the emission power of the AERA beacon is sufficient.

\subsubsection{Conclusions for AERA}

Several conclusions for the design of the AERA beacon system could be drawn by the test measurements :

- A beacon can improve the relative timing precision to $\lesssim 1$ ns making digital radio interferometry possible. Without a beacon, the relative timing precision of AERA stations is insufficient, because the GPS clocks of the individual AERA stations are not precise enough.

- Although the beacon signal is inside the frequency range used for data acquisition, it does not spoil the radio data analysis, because the beacon signal can be digitally filtered during subsequent data analysis.

- To achieve a sufficient improvement of the relative timing precision, a signal-tonoise ratio of the beacon signal of $\gtrsim 20 \mathrm{~dB}$ in power is required. To guarantee this over the whole extension of AERA, the beacon emission power has to be significantly higher than at LOPES.

- To obtain a homogeneous beacon amplitude over the entire AERA site, the beacon should be located as far away from AERA as possible. Still, the only feasible location which does not require major efforts is the communication tower of Coihueco. For AERA phase 1, this is an adequate choice, as the extension of the array $(\sim 800 \mathrm{~m})$ is small compared to its distance to Coihueco $(\sim 5 \mathrm{~km})$. When the extension of AERA grows (up to $\sim 7 \mathrm{~km}$ in phase 3 ) this might not be sufficient anymore.

\subsection{AERA Beacon system}

The conclusions from the test measurements have been considered in the design and development of the final beacon system. It has successfully been deployed in April 2010. Details about the design of the AERA beacon, its functionality, and performed tests of the beacon system can be found in the diploma thesis of M. Konzack [116], and in a technical GAP note 2010-099 of the Pierre Auger Observatory [117].

\subsubsection{Design and installation}

The AERA beacon system has been installed at the fluorescence telescope Coihueco, about $5 \mathrm{~km}$ west of the AERA phase 1 stations (cf., figure 3.7). Because the beacon design aims to provide continuously a stable sub-nanosecond timing precision, the beacon must work reliably under the conditions of the Argentinian Pampa (i.e., strong winds, occasional thunderstorms, power cuts), and should only rarely require 


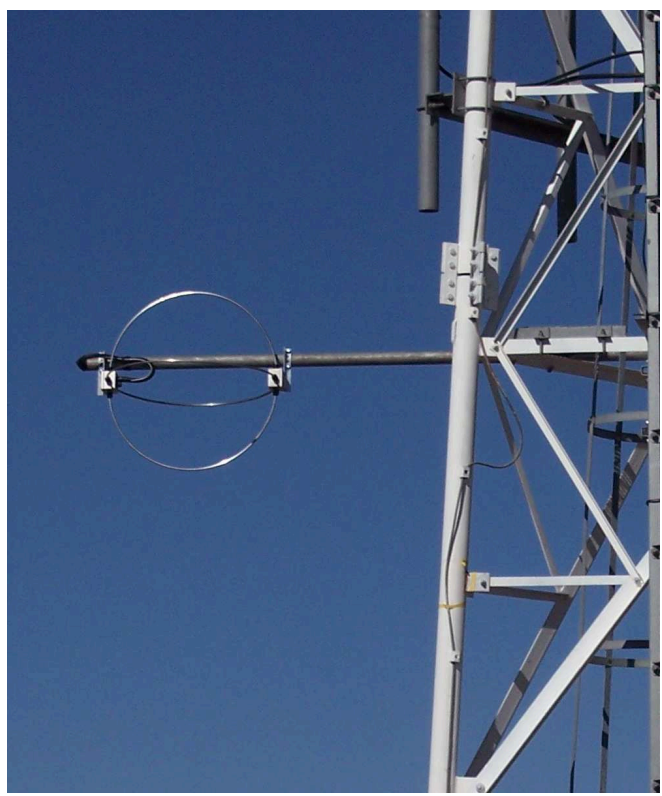

(a) beacon antenna

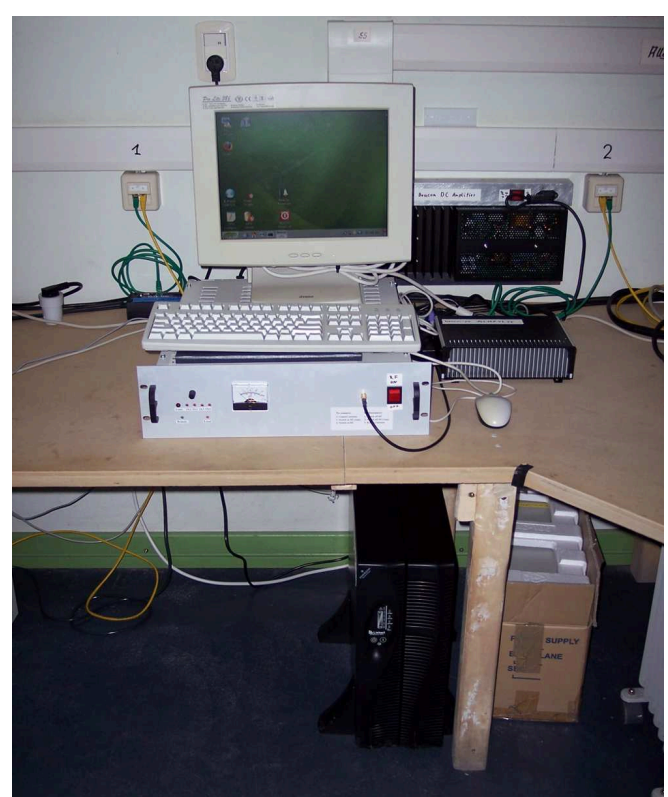

(b) beacon electronics

Figure 5.6.: Photographs of the AERA beacon system after its installation at the Coihueco telescope building in April 2010.

manual access for maintenance. The Coihueco telescope building has been chosen as deployment site because of the available infrastructure. This is a compromise, since an optimum location would be at greater distance. Since Coihueco is almost exactly in the west of AERA, the beacon signals are most pronounced in the north-south polarization channels of each individual AERA station.

The beacon system consists of an emitting antenna, electronics for signal generation, and a computer system to enable remote control and monitoring of the system (see figure 5.6), e.g., from the KIT in Germany. Thus, the diagnosis of possible problems and many settings, like switching the beacon on or off, or changing its emission power, can be done remotely. In addition, redundancies have been built into the system. For instance, the AERA beacon features the simultaneous emission of four frequencies, although LOPES has proven that in principle two are sufficient.

The following list gives a short description of the different beacon components. A schematic overview is shown in figure 5.7, and table 5.3 contains the most important technical specifications.

\section{- Antenna}

In principle, any broad band antenna is suitable for the beacon, but only limited space is available at the communication tower of Coihueco, and the antenna has to withstand frequent storms. Because of its small size and stability, a SALLA antenna has been chosen [99], and a special version for an input power up to $10 \mathrm{~W}$ was built. Thereby, two disadvantages were accepted. First, the emission pattern of the SALLA is broad, and the emission cannot be beamed 


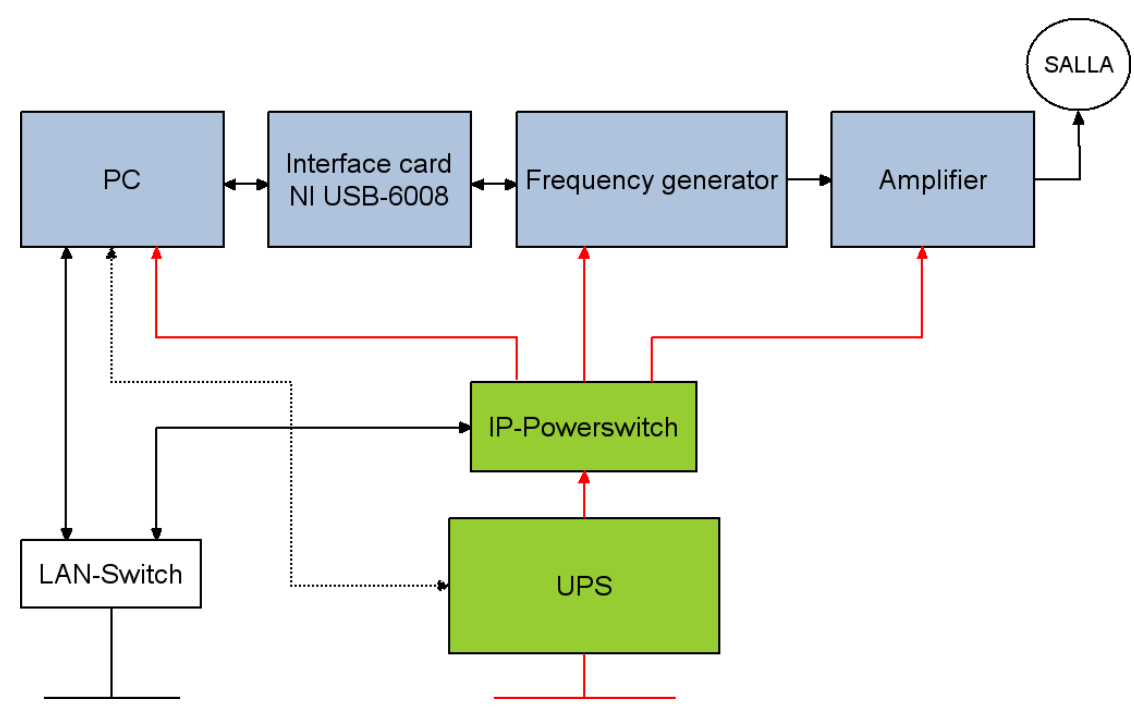

Figure 5.7.: Layout of the AERA beacon system: power lines are drawn in red, signal lines in black.

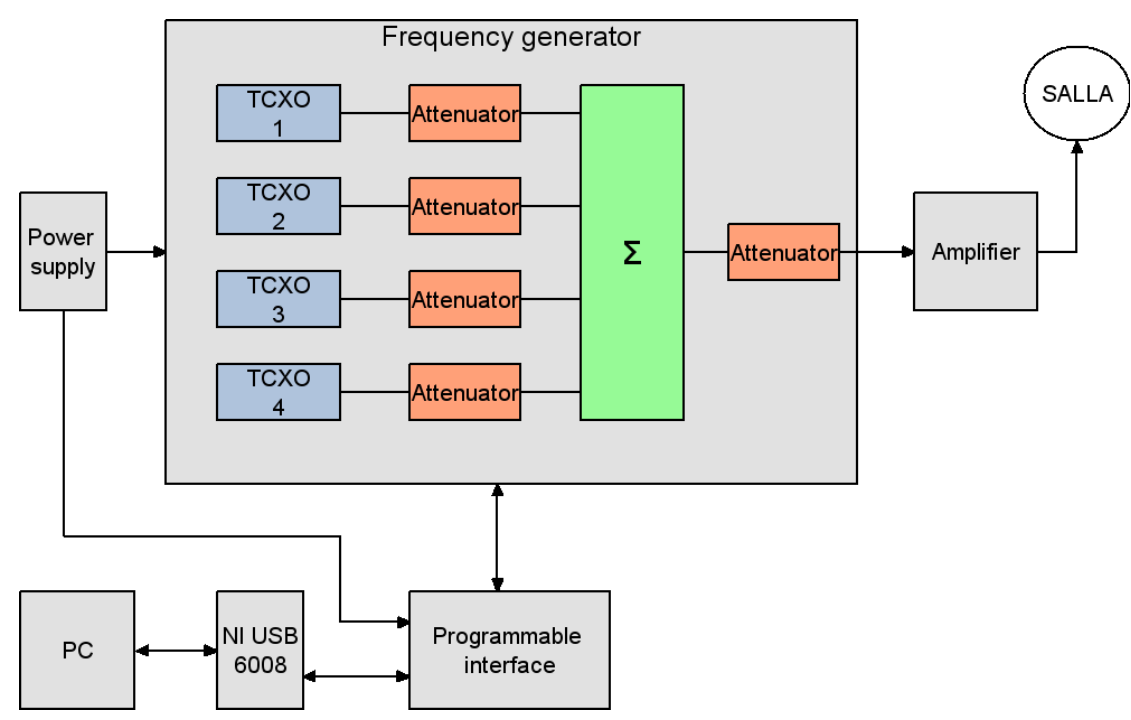

Figure 5.8.: Scheme of the beacon electronics: four frequencies are generated and attenuated separately which allows to individually control the power of each frequency. After mixing all frequencies, the total power can be controlled via a final attenuator. 
Table 5.3.: Technical specifications of the AERA beacon.

\begin{tabular}{|c|c|c|}
\hline \multirow[t]{10}{*}{ Beacon components } & Transmission antenna & SALLA $75 \mathrm{~cm}$ \\
\hline & Frequency generator & In-house design by KIT, IK \\
\hline & Amplifier & ZHL-5W-1 \\
\hline & Cable & RG $213,60 \mathrm{~m}$, attenuation $2.5 \mathrm{~dB}$ \\
\hline & Lightning protection & NexTek SurgeGuard \\
\hline & Computer & AR-ES5630FL \\
\hline & & (same type as for AERA DAQ) \\
\hline & Interface card & NI USB-6008 \\
\hline & UPS & GXT2-1000VA + battery extension \\
\hline & IP power switch & Leunig ePowerSwitch EPS 4 \\
\hline \multirow[t]{2}{*}{ Complete system } & Power consumption & $\sim 100 \mathrm{~W}$ \\
\hline & $\begin{array}{l}\text { Maximum emission power } \\
\text { with } 1,2,3 \text { or } 4 \\
\text { used frequencies }\end{array}$ & $\begin{array}{l}0.2 / 0.05 / 0.02 / 0.01 \mathrm{~W} \\
\text { per frequency, respectively }\end{array}$ \\
\hline \multirow[t]{4}{*}{ Antenna } & Type & $\begin{array}{l}\text { Short aperiodic loaded loop antenna } \\
\text { (special design for high power) }\end{array}$ \\
\hline & Size & Sphere with $75 \mathrm{~cm}$ diameter \\
\hline & Reflectivity at input & $-1.5 \mathrm{~dB}$ \\
\hline & Gain & $-10 \mathrm{~dB}$ \\
\hline \multirow[t]{9}{*}{ Frequency generator } & Frequency $f_{1}$ & $37.793 \mathrm{MHz}$ \\
\hline & Frequency $f_{2}$ & $46.582 \mathrm{MHz}$ \\
\hline & Frequency $f_{3}$ & $58.887 \mathrm{MHz}$ \\
\hline & Frequency $f_{4}$ & $71.191 \mathrm{MHz}$ \\
\hline & Frequency stability & $10^{-6}$ per year \\
\hline & Maximum output power & $1 \mathrm{~mW}$ \\
\hline & Parameter adjustments & Switch frequencies on/off \\
\hline & & Attenuation per frequency $(0-31 \mathrm{~dB})$ \\
\hline & & Common attenuation $(0-31 \mathrm{~dB})$ \\
\hline \multirow[t]{5}{*}{ Amplifier } & Amplification & $40 \mathrm{~dB}$ (fixed) \\
\hline & Frequency range & $5-500 \mathrm{MHz}$ \\
\hline & Maximum output power & $5 \mathrm{~W}$ \\
\hline & Maximum input power & $1 \mathrm{~mW}$ \\
\hline & Power consumption & $\sim 70 \mathrm{~W}$ \\
\hline \multirow[t]{4}{*}{$\overline{\mathrm{UPS}}$} & Manufacturer & Liebert \\
\hline & Nominal output power & $700 \mathrm{~W}, 1000 \mathrm{VA}$ \\
\hline & Battery & $4 \times 12 \mathrm{~V} \times 7 \mathrm{Ah}$ \\
\hline & Autonomous time & $\sim 400$ minutes \\
\hline \multirow[t]{4}{*}{ Beacon location } & Latitude & $35^{\circ} 06^{\prime} 51.92^{\prime \prime} \mathrm{S}$ \\
\hline & Longitude & $69^{\circ} 35^{\prime} 59.66^{\prime \prime} \mathrm{W}$ \\
\hline & Height & $6 \mathrm{~m}$ above the foundation of the \\
\hline & & Coihueco communication tower \\
\hline
\end{tabular}


into the direction of AERA. Second, the efficiency is low due to the internal resistor of the SALLA. According to measurements [118], only about $\sim 7 \%$ of the input power is emitted. Hence, the antenna design offers opportunities for future improvements of the beacon system.

- Cable

The antenna is connected by about $60 \mathrm{~m}$ of RG 213 cable, which attenuates the input power by about $2.5 \mathrm{~dB}$. Grounding of the cable shielding and a surge protection prevent damage by lightnings.

- Amplifier

The final amplification limits the maximum emission power. Taking into account the results of the test measurements, a $5 \mathrm{~W}$ amplifier has been chosen because this seems to be sufficient for AERA phase 1, and a major increase in power would be cost-driving. In the case of four beacon frequencies, a $5 \mathrm{~W}$ amplifier allows an output power of $5 \mathrm{~W} / 4^{2} \approx 0.3 \mathrm{~W}$ per frequency since the amplification is linear in the amplitude, i.e., the square root of the power.

- Signal generator (see figure 5.8)

The signal generator can emit up to four predefined frequencies whose power can be tuned individually. The frequencies have been chosen such that they do not interfere with previously measured background sources and coincide with frequency bins of an AERA spectrum. Their stability of $\leq 1 \mathrm{ppm} /$ year ensures that the maximum timing precision achievable with the beacon will not be limited by the beacon system itself, but instead by the AERA station design, noise or systematic effects.

- Universal power supply (UPS)

In the case of a power cut, the solar powered AERA DAQ will continue to take data. Hence, a UPS with an autonomous time of about $5 \mathrm{~h}$ will bridge the frequently occurring short power cuts at Coihueco. Only longer power cuts will lead to a controlled shut down of the beacon and a loss of the nanosecond relative timing precision for the AERA data.

- Computer system

Remote control and monitoring of the beacon system is done over a special interface card connected to a computer. For this, the same kind of computer as for the AERA DAQ has been chosen which includes no moving parts and is dust-proof. An in-house developed software handles the communication of the beacon with the CRS and the outer world [116].

- IP power switch

In the so far not experienced situation that neither the computer nor the electronics react, their power can cycled via an independent device connected to the Coihueco network. 


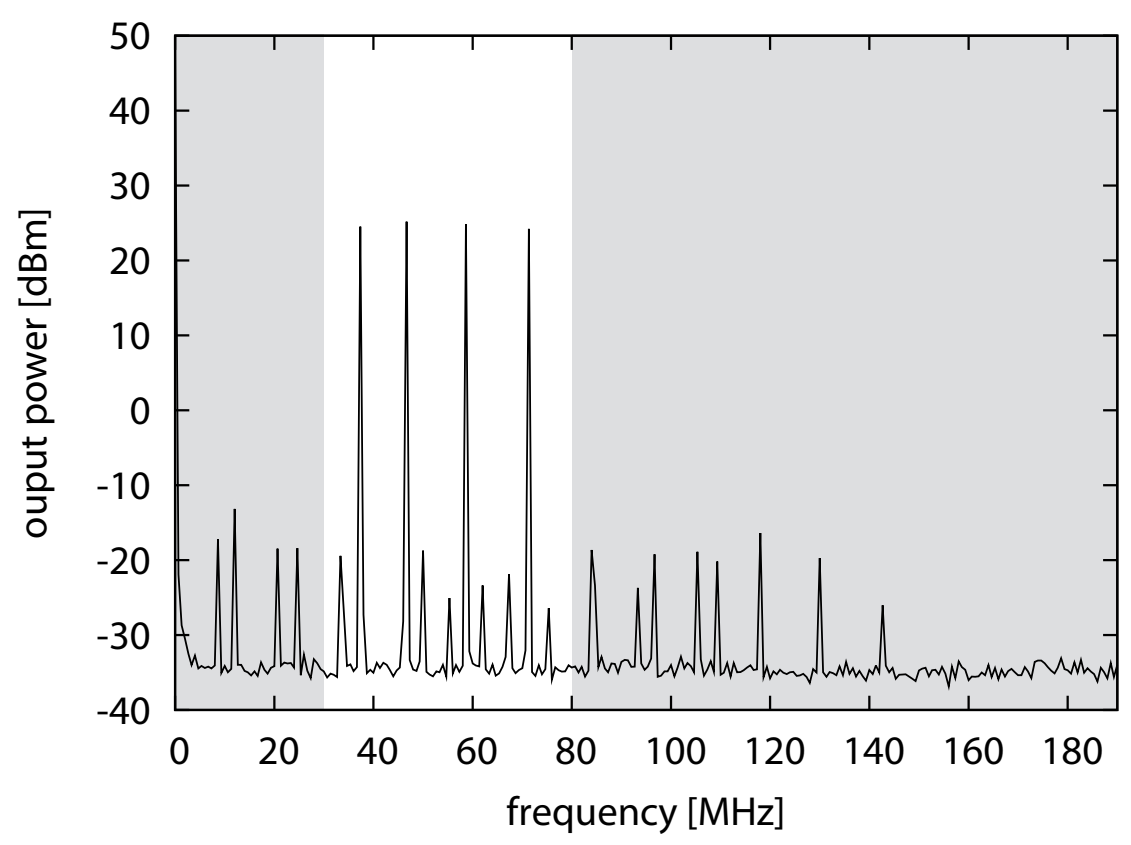

Figure 5.9.: Spectrum after the beacon amplifier. The power of the intermodulation peaks is at least $10 \mathrm{~dB}$ below the noise level at the AERA site. In addition, the intermodulation peaks outside of the AERA frequency range $(30-80 \mathrm{MHz})$ are not emitted by the beacon antenna, but suppressed by a bandpass filter between the amplifier and the cable to the antenna.

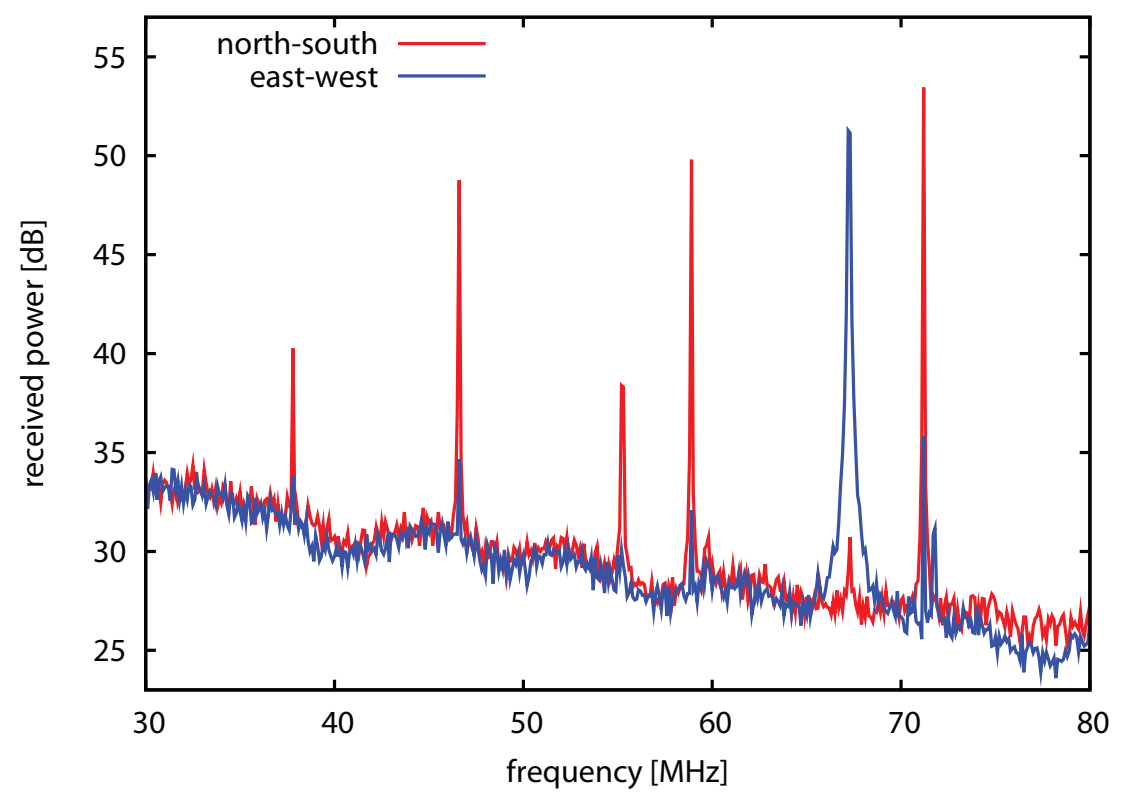

Figure 5.10.: Mean spectrum measured with AERA station 01, averaged over 100 events $(\sim 15 \mathrm{~min})$. The beacon signals are clearly visible in the northsouth polarization channel. The broad line in the east-west polarization and the line at $\sim 56 \mathrm{MHz}$ in the north-south polarization are narrow band RFI. 


\subsubsection{Performance of the beacon}

Several tests of the beacon functionality have been performed [116], covering the computer system and its software, the frequency generator and the overall system. Furthermore, analysis modules have been included in the Offline software framework (cf., section 3.3.3) to measure and improve the relative timing precision of AERA.

It was verified that the beacon electronics is indeed able to generate all four frequencies simultaneously, and with about the same power of $25 \mathrm{dBm}$ per frequency (see figure 5.9). The width of the frequency peaks is smaller than $100 \mathrm{~Hz}$, and below the resolution of the available spectrum analyzer. However, measuring the width more precisely is not necessary, since already a width of $100 \mathrm{~Hz}$ is good enough to achieve a sub-nanosecond timing precision. Nevertheless, the tests showed a minor problem, which could be resolved: The amplifier is not fully linear and produces intermodulation. While intermodulation peaks outside of the AERA frequency range are suppressed by a band pass filter, intermodulation peaks within the AERA frequency range are at least $40 \mathrm{~dB}$ lower than the amplitude of the main beacon frequencies. Since the signal-to-noise ratio of the beacon signal is about $20-30 \mathrm{~dB}$ at the AERA stations (see figure 5.10), the power of the intermodulation peaks is at least $10 \mathrm{~dB}$ below the noise level and does not disturb the operation of AERA.

The two most important tests were to check that the beacon signal can be continuously received at all AERA stations, and that the relative timing precision can indeed be improved to $\lesssim 1 \mathrm{~ns}$. Therefore, one of the first AERA commissioning runs was evaluated. It includes 19 AERA stations, simultaneously triggered with a periodic $0.1 \mathrm{~Hz}$ trigger. As expected, the beacon signal is continuously visible in the northsouth polarization channels of all AERA stations (see figure 5.11 for an example). The signal-to-noise ratio is sufficient $(\gtrsim 20 \mathrm{~dB})$ at all beacon frequencies, except at $f_{1}$. Thus, the AERA beacon will be operated with three frequencies $\left(f_{2}, f_{3}, f_{4}\right)$.

To determine by how much the beacon can improve the relative timing precision of AERA, the precision has been measured before and after improvement. The phase differences at the beacon frequencies have been determined with Offline, and converted to time differences between the individual AERA stations, choosing AERA station 01 as reference. The standard deviation of the time differences is the relative timing precision. Before improvement with the beacon, it is $2.3 \pm 0.2 \mathrm{~ns}$ (see table 5.4 ), which is consistent with the result of the test measurements at the MAXIMA setup (see section 4.6).

In a second step, each event has been corrected for the time difference measured at $f_{4}$ (the frequency with the highest signal-to-noise ratio). After this improvement, the relative timing precision has been measured again at $f_{2}$ and $f_{3}$. The result at $f_{3}$ is believed to be more precise, since $f_{3}$ has a higher signal-to-noise ratio: The relative timing precision after improvement is $0.5 \pm 0.1 \mathrm{~ns}$. This is better than the requirement of $1 \mathrm{~ns}$, which leaves a safety margin for systematic effects like they have been observed with LOPES in the order of $\sim 0.5 \mathrm{~ns}$.

Concluding, the tests showed that the AERA beacon works as expected, and is fully sufficient for AERA phase 1. An improvement of the relative timing precision is possible in a subsequent analysis, e.g., in Offline, for each event which contains the beacon signal. Thus, running the beacon continuously allows using AERA as digital radio interferometer. 


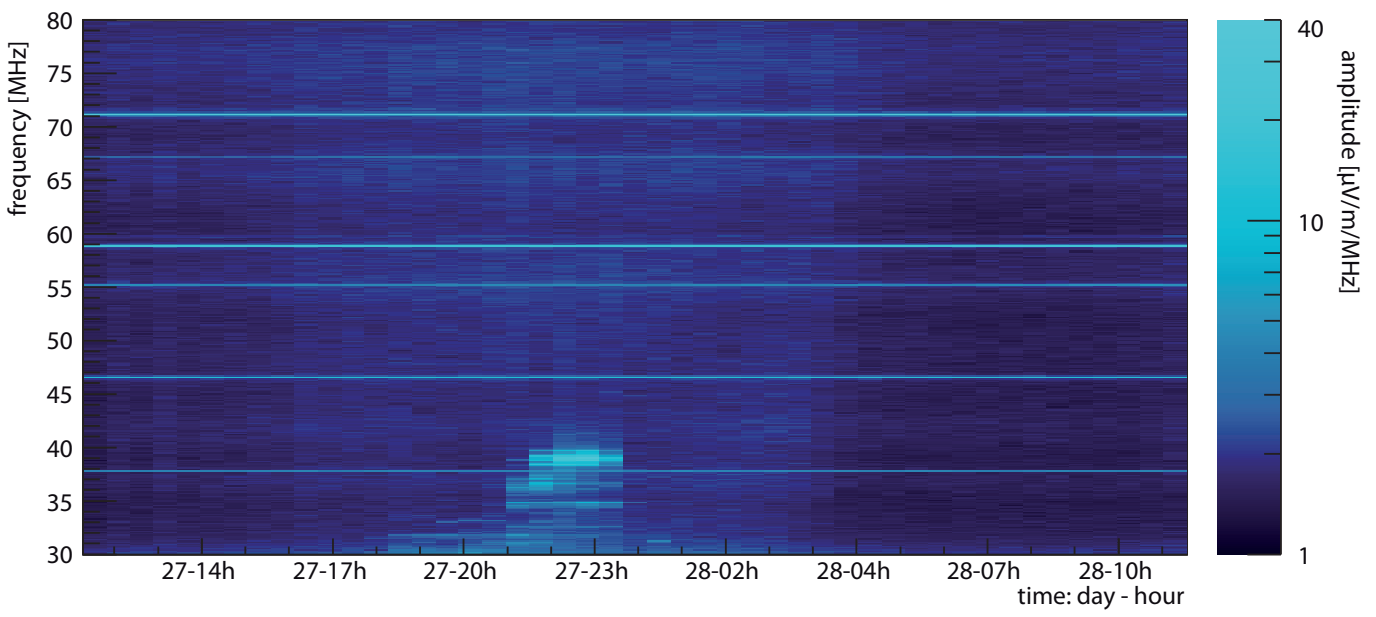

Figure 5.11.: Dynamic spectrum measured with the north-south polarization of AERA station 01, recorded during 27/28 October 2010. The beacon signals are visible as horizontal lines. The noise level shows a modulation with sidereal time, since the noise is partly of galactic origin.

Table 5.4.: AERA timing precision, measured relative to station 01. The measurement was done with the second and third beacon frequency $\left(f_{2}=\right.$ $46.582 \mathrm{MHz}, f_{3}=58.887 \mathrm{MHz}$ ), after and before improvement of the timing with the fourth beacon frequency $\left(f_{4}=71.191 \mathrm{MHz}\right)$. Due to the better signal-to-noise ratio, the measurement at $f_{3}$ is more precise.

\begin{tabular}{ccccc}
\hline AERA station & \multicolumn{2}{c}{ before improvement } & \multicolumn{2}{c}{ after improvement } \\
& $f_{2}$ & $f_{3}$ & $f_{2}$ & $f_{3}$ \\
\hline 02 & $2.28 \mathrm{~ns}$ & $2.30 \mathrm{~ns}$ & $0.76 \mathrm{~ns}$ & $0.48 \mathrm{~ns}$ \\
03 & $2.65 \mathrm{~ns}$ & $2.63 \mathrm{~ns}$ & $0.84 \mathrm{~ns}$ & $0.54 \mathrm{~ns}$ \\
04 & $2.04 \mathrm{~ns}$ & $2.02 \mathrm{~ns}$ & $0.53 \mathrm{~ns}$ & $0.40 \mathrm{~ns}$ \\
05 & $2.23 \mathrm{~ns}$ & $2.24 \mathrm{~ns}$ & $0.53 \mathrm{~ns}$ & $0.40 \mathrm{~ns}$ \\
06 & $2.19 \mathrm{~ns}$ & $2.21 \mathrm{~ns}$ & $0.54 \mathrm{~ns}$ & $0.39 \mathrm{~ns}$ \\
08 & $2.30 \mathrm{~ns}$ & $2.29 \mathrm{~ns}$ & $0.57 \mathrm{~ns}$ & $0.41 \mathrm{~ns}$ \\
09 & $2.15 \mathrm{~ns}$ & $2.13 \mathrm{~ns}$ & $0.56 \mathrm{~ns}$ & $0.41 \mathrm{~ns}$ \\
10 & $2.39 \mathrm{~ns}$ & $2.37 \mathrm{~ns}$ & $0.70 \mathrm{~ns}$ & $0.46 \mathrm{~ns}$ \\
11 & $2.34 \mathrm{~ns}$ & $2.15 \mathrm{~ns}$ & $1.06 \mathrm{~ns}$ & $0.46 \mathrm{~ns}$ \\
12 & $2.52 \mathrm{~ns}$ & $2.64 \mathrm{~ns}$ & $0.75 \mathrm{~ns}$ & $0.80 \mathrm{~ns}$ \\
13 & $2.32 \mathrm{~ns}$ & $2.33 \mathrm{~ns}$ & $0.73 \mathrm{~ns}$ & $0.47 \mathrm{~ns}$ \\
15 & $2.41 \mathrm{~ns}$ & $2.44 \mathrm{~ns}$ & $0.72 \mathrm{~ns}$ & $0.45 \mathrm{~ns}$ \\
16 & $2.54 \mathrm{~ns}$ & $2.40 \mathrm{~ns}$ & $1.11 \mathrm{~ns}$ & $0.57 \mathrm{~ns}$ \\
17 & $2.56 \mathrm{~ns}$ & $2.37 \mathrm{~ns}$ & $1.19 \mathrm{~ns}$ & $0.70 \mathrm{~ns}$ \\
18 & $2.32 \mathrm{~ns}$ & $2.33 \mathrm{~ns}$ & $0.70 \mathrm{~ns}$ & $0.45 \mathrm{~ns}$ \\
19 & $2.59 \mathrm{~ns}$ & $2.50 \mathrm{~ns}$ & $0.70 \mathrm{~ns}$ & $0.48 \mathrm{~ns}$ \\
20 & $2.35 \mathrm{~ns}$ & $2.37 \mathrm{~ns}$ & $0.88 \mathrm{~ns}$ & $0.47 \mathrm{~ns}$ \\
21 & $2.13 \mathrm{~ns}$ & $2.12 \mathrm{~ns}$ & $0.53 \mathrm{~ns}$ & $0.40 \mathrm{~ns}$ \\
\hline Mean & $2.35 \mathrm{~ns}$ & $2.32 \mathrm{~ns}$ & $0.75 \mathrm{~ns}$ & $0.48 \mathrm{~ns}$ \\
$\mathrm{RMS}$ & $0.17 \mathrm{~ns}$ & $0.17 \mathrm{~ns}$ & $0.20 \mathrm{~ns}$ & $0.11 \mathrm{~ns}$ \\
& & & &
\end{tabular}




\subsection{Outlook}

The AERA reference beacon has successfully been installed at Coihueco and is ready for use. Although the beacon has been tested to perform very well, there is still room for improvements with respect to its application. Experience over a longer time period ( $\sim 1$ year) will be necessary to study systematic effects and the long time stability of the beacon system. Then, the emission power of each beacon frequency can be fine tuned to optimize the beacon performance.

Basic functionality to manually improve the relative timing of individual AERA events is already available in Offline. On the long term it is foreseen to automate the improvement of the relative timing precision. This includes handling time-varying reference phase differences in databases, and to optimize the algorithm for the beacon correction compared to the one used in LOPES. This is challenging because the situation of AERA is more complex: At LOPES, the beacon has 'only' the purpose to monitor drifts and jumps of the relative timing, but at AERA, the GPS timing precision has to be improved on a per-event basis. Furthermore, not every AERA station is present in every event. Thus, it is not possible to base the AERA beacon improvement on a fixed reference station. Instead, the reference station has to be defined for each event by an automated algorithm. Moreover, the time scale for which the reference phase differences are valid must still be investigated. This will be possible, once data over a reasonable period ( $\gtrsim 1$ year) will have been recorded. Then, also systematic effects and the impact of the beacon on an interferometric analysis pipeline can be studied. The implementation of this automated beacon correction into Offline has to be accompanied by the implementation of an interferometric analysis pipeline, to evaluate the prospects of an interferometric analysis. Due to delays in the built-up of AERA, this could unfortunately not be performed for this thesis.

Another task will be to include the beacon into the AERA monitoring system. Since the beacon provides a continuous reference signal with defined amplitude and phasing, any failures of AERA stations can presumably be detected. For example, a changing gain will lead to a change in the measured beacon amplitude, or an accidental mis-cabling or mis-alignment of an antenna will lead to a change in the measured phasing. This monitoring method has already been useful at LOPES.

Last but not least, the total relative timing precision and accuracy, including not only the GPS clocks, but also the delays of all components (cables, filters, etc.) have to be checked. This can be done with calibration measurements, either with astronomical sources or a pulse generator like at LOPES. Finally, the evaluation of AERA phase 1 will show to which extent the beacon will improve the reconstruction of the primary cosmic ray properties. Then, possible upgrades of the beacon for AERA phase 2 or 3 can be planned. This might be advisable because the power of the current beacon system will probably not be sufficient for AERA phase 3 . The experience obtained from the AERA beacon will also help with the design of future radio observatories for air shower detection, i.e., under which conditions the additional effort of a beacon is worth paying for. 


\section{Treatment of Noise}

The presence of radio noise affects the measurement of the air shower induced radio pulse at each antenna and predominantly determines the error of the measurement. Noise can interfere constructively or destructively with the radio pulse and increases or decreases the pulse amplitudes. On average noise increases the measured pulse amplitudes since the power of the noise adds to the pulse power. It will be shown that this effect depends on the signal-to-noise ratio, and that noise systematically flattens the lateral distribution of the radio signal. Therefore, an adequate treatment of noise is especially important for experiments in a noisy environment, like LOPES.

This chapter starts with an explanation why the situation at LOPES is different from both communication engineering and normal particle physics experiments. The special situation of air shower radio measurements has already been investigated in the frame of self trigger development [81], where the signal-to-noise ratio plays the role of a threshold. Handling noise in data analysis is more complex, and is the topic of this chapter. Special attention has to be paid to measure signal and noise consistently with each other. In principal, this is a trivial statement, but so far it has been neglected by digital radio experiments for cosmic ray detection.

After a general introduction into the topic, a new method for noise measurement is presented, which is consistent with the method used at LOPES to measure pulse amplitudes at individual antennas. The new method is compared to other methods for noise measurements used in the field of cosmic ray radio detection. Based on an analysis with test pulses and real noise, an approach is presented how to correct for noise, and how to determine the noise dependent error of the amplitude and time measurements at individual antennas. The influence of noise on the cross-correlation beam analysis is not in the scope of the present studies. ${ }^{1}$

\subsection{Noise and RFI}

There are two kinds of disturbances when measuring radio signals: noise and radio frequency interferences (RFI). They can be both of natural and technical origin, and there is a smooth transition between both terms. In this thesis, they are distinguished as follows:

Noise is always uncorrelated with the signal, and has no distinct characteristics. Noise can be of thermal origin, for instance, internal noise of the data acquisition electronics, or noise from the cosmic microwave background. However, also other radio perturbations with indistinct characteristics are usually called noise and described by a noise temperature, even if they are not or only partially of thermal origin. Examples are the galactic radio background which has a noise temperature

\footnotetext{
${ }^{1}$ A summary of this chapter is published in Nuclear Instruments and Methods A 662 (2012) S238S241 [119].
} 


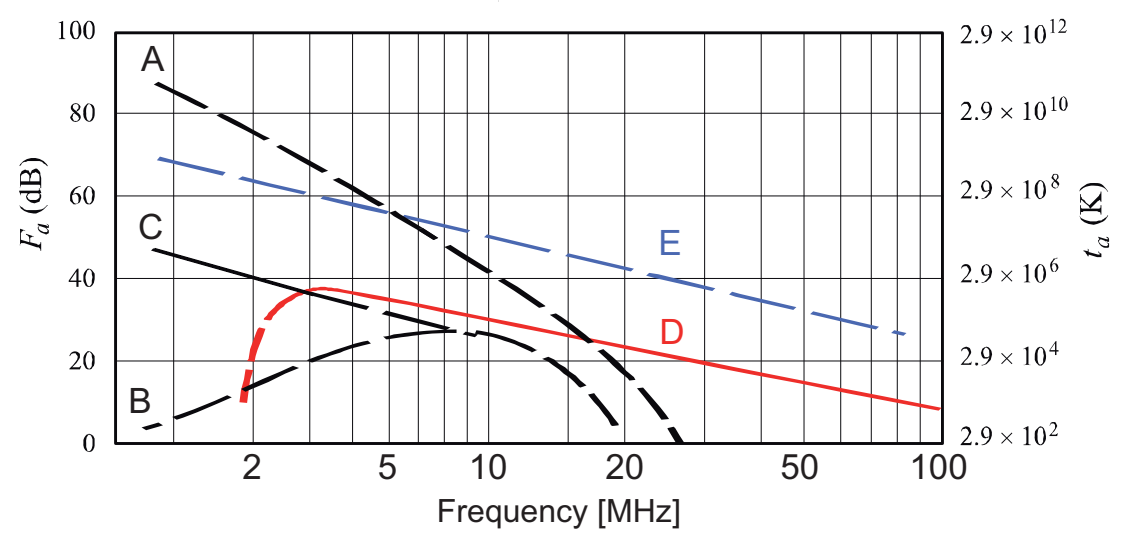

A atmospheric noise, value exceeded $0.5 \%$ of time
B atmospheric noise, value exceeded $99.5 \%$ of time
C man-made noise, quiet receiving site
D galactic noise
E median city area man-made noise
minimum noise level expected

Figure 6.1.: Radio background at $\mathrm{MHz}$ frequencies (power and temperature), extracted from reference [120]. For LOPES, the background is dominated by anthropogenic noise (curve E). At AERA, the background level is lower and expected to be dominated by galactic noise (curve D).

of a few $1,000 \mathrm{~K}$ in the LOPES band $(40-80 \mathrm{MHz})$, and anthropogenic noise with a temperature of several $10,000 \mathrm{~K}$ in urban areas (see figure 6.1) [121, 120]. Hence, anthropogenic noise is the dominant noise component at LOPES. It is a sum of numerous radio emissions from any technical apparatuses (cars, electronics, machines generating sparks), which cannot be resolved as individual sources. Due to the large number of contributing noise sources, noise is thought to be approximately Gaussian distributed. However, due to the non-random nature of many noise sources, noise does not necessarily follow a strict Gaussian distribution. Indeed, experience with self-trigger development for AERA shows that the chance for fluctuations to high noise amplitudes is larger than expected from a Gaussian distribution.

RFI, in contrast to noise, has distinct characteristics. Narrow band RFI usually originates from radio communication. For instance, until end of 2007, the most prominent RFI source for LOPES was the carrier of a television transmitter (see figure 6.2 (a)). Because the air shower radio pulse is broad band, any narrow band RFI can be digitally filtered without disturbing the radio pulse measurement. Only narrow band RFI whose amplitude is too low to be resolved in the spectrum, will remain in the filtered radio data and contribute to the anthropogenic radio noise.

Furthermore, there is broad band RFI, i.e., radio pulses which do not originate from the air shower. Such RFI pulses are generated by natural sources like thunderstorms, as well as technical sources, and sometimes referred to as 'transient noise'. Most LOPES events do not exhibit any significant radio pulse at all - neither an air shower pulse nor an RFI pulse. Thus, the chance probability of an RFI pulse in a LOPES event exactly at the air shower time is close to zero, and the influence of RFI pulses on LOPES data is neglected. The most prominent broad band RFI source is 


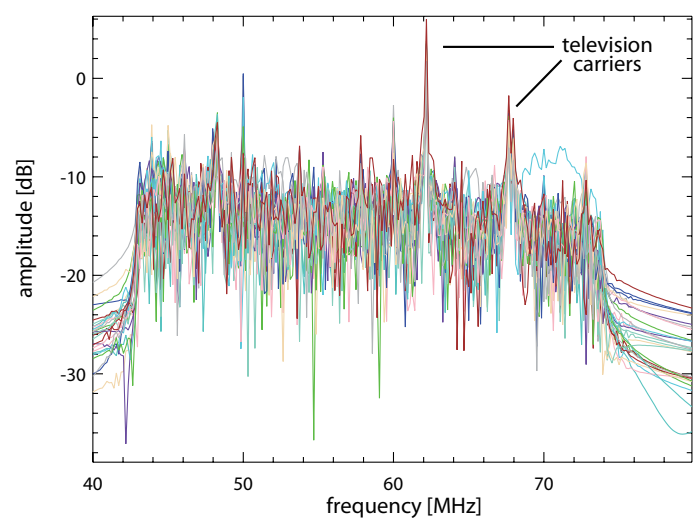

(a) frequency spectrum

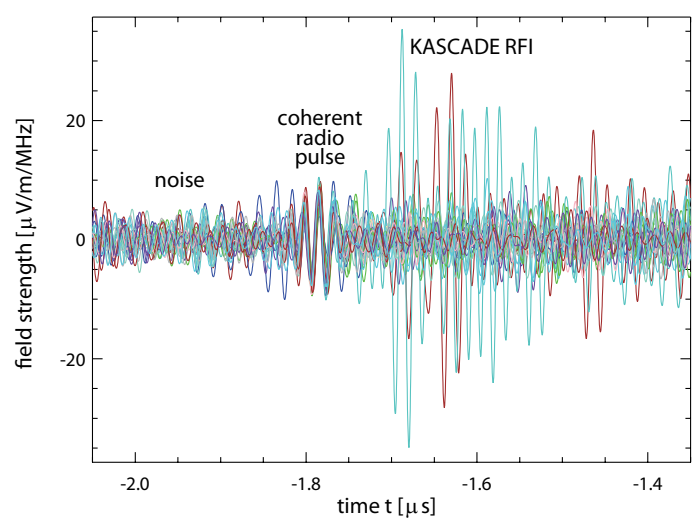

(b) time series

Figure 6.2.: Typical LOPES event measured with 26 antennas (different colors). (a) Narrow band RFI can clearly be distinguished in the frequency spectrum, and is filtered for data analysis. The amplitude is suppressed outside the effective bandwidth of the bandpass filters $(43-74 \mathrm{MHz})$. (b) In contrast to the air shower radio pulse, noise and the KASCADE RFI are oscillating incoherently at individual antennas. Nevertheless the oscillation frequency is always similar, because it is determined by the LOPES bandpass filter.

the scintillator electronics of KASCADE. This KASCADE RFI occurs always after the air shower radio pulse, and does not disturb the measurement of the radio pulse amplitude, because it only overlaps with the falling edge of the radio pulse. However, the KASCADE RFI disturbs the measurement of the integrated pulse power. This is one important reason why the maximum instantaneous amplitude, but not the integrated power of the radio pulse is taken as signal measurement. The second reason is that the signal-to-noise ratio is maximum at the maximum of the pulse.

The identification of the air shower radio pulse is a challenge, in particular when the signal-to-noise ratio is very low - often close to 1 in individual antennas. In such cases, the radio pulse can only be identified by an interferometric combination of all LOPES antennas, i.e., by cross-correlation beamforming (cf. section 3.2.2). After beamforming, the air shower radio pulse can be distinguished from noise and the KASCADE RFI, because the oscillation caused by the bandpass filter occurs coherently in all antennas at the radio pulse, but is uncorrelated for noise and RFI (see figure $6.2(\mathrm{~b})$ ). Therefore, in the LOPES analyses presented in chapters 7 and 8 , a cut is made on the signal-to-noise ratio of the cross-correlation amplitude as well as on the amount of the coherent power in the pulse. The coherent power is required to be larger than $80 \%$ of the total power, because by chance also noise can be partially coherent, especially as the beamforming direction is optimized by a fit which maximizes the cross-correlation amplitude (cf. section 4.2 for details). Once the exact pulse time has been identified with the cross-correlation beam, the pulse amplitude at that time can be measured in each individual antenna, even at low signal-to-noise ratios. Thus, the influence of noise on pulse amplitudes has also been 
evaluated for low signal-to-noise ratios, where the behavior is not trivial.

From communication engineering, thresholds effects are known for low signal-tonoise ratios, for instance, with respect to signal demodulation, e.g., for amplitude or frequency modulated signals [122]. Although the analogy is not complete, since LOPES does not measure modulated signals, we will see that one basic characteristic is preserved. Also for the detection of air shower radio signals, the influence of noise is different at low and high signal-to-noise ratios. Hence, special effort has to be made, if measurements of antennas with high and low signal-to-noise ratios shall be combined to analyze the data on the basis of single antennas.

Summarizing, for the analysis of radio signals, we have to distinguish between perturbation by RFI and radio noise. At LOPES, RFI does not influence the air shower radio pulse, because it is either filtered, or, in the case of RFI from KASCADE, can be distinguished by its arrival time. Consequently, any remaining disturbance of the air shower radio pulse is due to noise, which at LOPES is mostly anthropogenic. Since anthropogenic noise is difficult to model realistically, the effect of noise on data is best analyzed with measurements of real noise, and not by any noise models or simulations.

\subsection{Measurement of signal and noise with LOPES}

Methods for noise measurement applied so far in the field of cosmic ray radio detection are originating from communication engineering. There, a signal usually has a power much larger than the noise, and lasts for a time significantly longer than its oscillation period ( $\approx 17 \mathrm{~ns}$ for the LOPES bandpass filter). Both is not true for air shower induced radio pulses. A simple measurement of the average noise power would only be sufficient, if the radio signal would be defined as the integrated power of the radio pulse.

However, due to the low signal-to-noise ratio of most air shower radio pulses, and because of the KASCADE RFI, a measurement of the integrated pulse power is not feasible for LOPES. For other radio experiments which do not experience the problem of subsequent RFI after the radio pulse, defining the signal as integrated power of the pulse is a tempting approach, since this method could be realized directly in analog electronics. However, this approach has the essential disadvantage that the integration interval would also include time regions in which the noise is dominant, because the width of radio pulse is usually not (much) larger than the reciprocal of the observing frequency (depending on the observing distance, cf. section 2.4). Consequently, measuring the integrated pulse power would decrease the signal-tonoise ratio compared to a signal measurement at the maximum of the pulse, and thus leads to a higher detection threshold, and reduces the number of events. ${ }^{2}$

At LOPES, the signal in individual antennas is defined at the maximum instantaneous amplitude, i.e., the maximum of a Hilbert envelope of the up-sampled electric field strength trace (cf. appendix B $)^{3}$. Consequently, also noise has to be measured in

\footnotetext{
${ }^{2}$ For experiments aiming at the detection of molecular bremsstrahlung at $\mathrm{GHz}$ frequencies, measuring the integrated pulse power will not significantly change the signal-to-noise ratio and the detection threshold, since the pulse duration is expected to be much longer than the reciprocal of the observing frequency.

${ }^{3}$ In previous LOPES analyses ([34] and [47]) the signal in individual antennas has been defined
} 
a consistent way, which is different from what is used in communication engineering. For this reason, a new method for noise measurement has been investigated, which is consistent with the signal definition.

The consistency of any method for signal and noise measurement can be expressed by the following consistency criterion for the measured signal-to-noise ratio of pure noise:

$$
\text { for } \text { true signal }=0 \quad \longrightarrow \quad \frac{\text { measured signal }}{\text { noise }} \stackrel{!}{=} 1 \quad \text { (on average) }
$$

The measured signal contains always also the noise, while the 'true signal' is the contribution of the air shower induced radio pulse. Hence, the measured signalto-noise ratio does not tend to 0 for a vanishing signal amplitude, but should on average be 1 . In the following, the true signal will be called $A_{\text {true }}$, and the measured signal-to-noise ratio $A_{\text {meas }}$, i.e., $A_{\text {meas }}$ is the measured signal normalized to the noise level.

Since the electric field is a vectorial quantity, the instantaneous electric field vector of the noise can be (partially) parallel or anti-parallel to the electric field vector of the signal at a certain time. Thus, noise can interfere constructively or destructively with the air shower radio emission, and increase or decrease the measured signal compared to the true signal. This is completely different to normal particle physics experiments, where noise always increases the measured signal. At LOPES, even for a positive true signal $A_{\text {true }}>0$, the measured signal-to-noise ratio $A_{\text {meas }}$ can in some cases be smaller than one. In addition, fluctuations of the noise level can also result in $A_{\text {meas }}<1$, as the noise measurement is performed in a $10 \mu \mathrm{s}$ time interval slightly before the signal (10.5 $-0.5 \mu$ s before the signal). Therefore, the consistency criterion is only supposed to hold on average.

Because the signal is the maximum amplitude of the pulse, a consistent method for noise measurement has to yield the average amplitude in a noise time window. This sounds trivial, but the difficulty is in defining the exact algorithm to calculate the average amplitude in the noise window. So far, various methods have been used for analyses of LOPES and AERA data, which turned out to be inconsistent with the method for signal measurement at individual antennas. Several methods for noise measurements (see table 6.1) have been tested with LOPES events using the procedure described in table 6.2. For instance in reference [47], the mean of the absolute of the field strength trace has been used as noise level (method 1). For analyses with the radio prototype setups at the Pierre Auger Observatory, sometimes the RMS of either the field strength trace or the power has been used (methods 2 and 6). None of these methods is consistent with the signal definition used at LOPES (nor with the similar signal definition used at AERA). Method 6 is expected to fulfill the consistency criterion if the signal is defined as integrated pulse power, but it does not fulfill the consistency criterion when the signal is defined as maximum amplitude of the pulse (see table 6.4).

as the maximum absolute field strength. This is almost but not exactly equal to the maximum of the Hilbert envelope, which typically exceeds the maximum absolute field strength by a few percent. 
Table 6.1.: Methods tested for noise measurement; all methods work on the upsampled field strength trace $\epsilon(t)$. Method 5 has been chosen as default for the LOPES standard analysis pipeline, because it is the only one consistent with the method for signal measurement (see text for details).

\begin{tabular}{ll}
\hline 1 & mean of the absolute of the trace \\
2 & standard deviation $^{b}$ of the trace, i.e., RMS of $\epsilon(t)$ \\
3 & mean of the local maxima of the absolute field strength $|\epsilon(t)|$ \\
4 & mean of the local maxima of a Hilbert envelope of the trace \\
$\mathbf{5}$ & weighted average of the local maxima of an envelope of the trace \\
6 & square root of the standard deviation of the power, i.e., $\sqrt{\operatorname{RMS}\left(\epsilon^{2}(t)\right)}$ \\
\hline
\end{tabular}

\footnotetext{
${ }^{a}$ A similar method would be to measure the mean of the Hilbert envelope of the trace. This method has not explicitly tested. It is not consistent with the used signal definition, either, because it would result in smaller values than the consistent method 5 .

${ }^{b}$ Following the language use in physics, 'RMS' is used as synonym for 'standard deviation', although the exact mathematical definition is different.
}

Table 6.2.: Procedure for testing the consistency criterion for noise measurement with LOPES events:

- 200 LOPES events without strong radio pulses (e.g., from air showers or broad band RFI) have been selected, covering different days and hours of the day. 100 of these events have been measured with the dual polarization setup, 100 with the LOPES 3D setup.

- All calibration and data conditioning steps from the standard analysis pipeline (e.g., suppression of narrow band RFI) have been applied.

- 20 noise time windows of $10 \mu \mathrm{s}$ each have been selected. The first and last eighth of each event has been skipped, since it is affected by the antialiasing filter of the standard analysis pipeline. The windows are not overlapping and have a distance of 5 us to each other.

- For each noise window, the noise level has been calculated for all 30 antennas six times, testing the six different methods of table 6.1. This way, 600 noise values could be obtained for each noise calculation method, for each of the 200 events.

- The signal has been measured for each noise window as the local maximum of a Hilbert envelope which is closest to the middle of the noise window. As there is no real signal in the noise windows, this corresponds to a measurement of a true signal $A_{\text {true }}=0$.

- The mean and the standard deviation of the measured signal-to-noise ratios $A_{\text {meas }}$ for $A_{\text {true }}=0$ have been calculated for each method.

- For the investigation of systematic effects, the analysis has been repeated for different up-sampling rates and sizes of the noise window. 
Table 6.3.: Signal-to-noise ratios of pure noise, i.e., $A_{\text {true }}=0$, for different methods: mean and standard deviation (RMS). The given uncertainties are the semi-difference between the results obtained with the LOPES dual polarization setup and the LOPES 3D setup.

\begin{tabular}{cccc}
\hline Method & Mean SNR(noise) & RMS(SNR) & RMS/SNR \\
\hline 1 & $2.102 \pm 0.034$ & $0.891 \pm 0.011$ & $(42.4 \pm 0.2) \%$ \\
2 & $1.667 \pm 0.027$ & $0.705 \pm 0.008$ & $(42.3 \pm 0.2) \%$ \\
3 & $1.365 \pm 0.021$ & $0.579 \pm 0.006$ & $(42.4 \pm 0.2) \%$ \\
4 & $1.049 \pm 0.008$ & $0.446 \pm 0.001$ & $(42.5 \pm 0.2) \%$ \\
$\mathbf{5}$ & $\mathbf{1 . 0 0 2} \pm \mathbf{0 . 0 0 1}$ & $\mathbf{0 . 4 2 5} \pm \mathbf{0 . 0 0 2}$ & $\mathbf{( 4 2 . 4} \pm \mathbf{0 . 2}) \boldsymbol{\%}$ \\
6 & $1.252 \pm 0.020$ & $0.530 \pm 0.005$ & $(42.4 \pm 0.3) \%$ \\
\hline
\end{tabular}

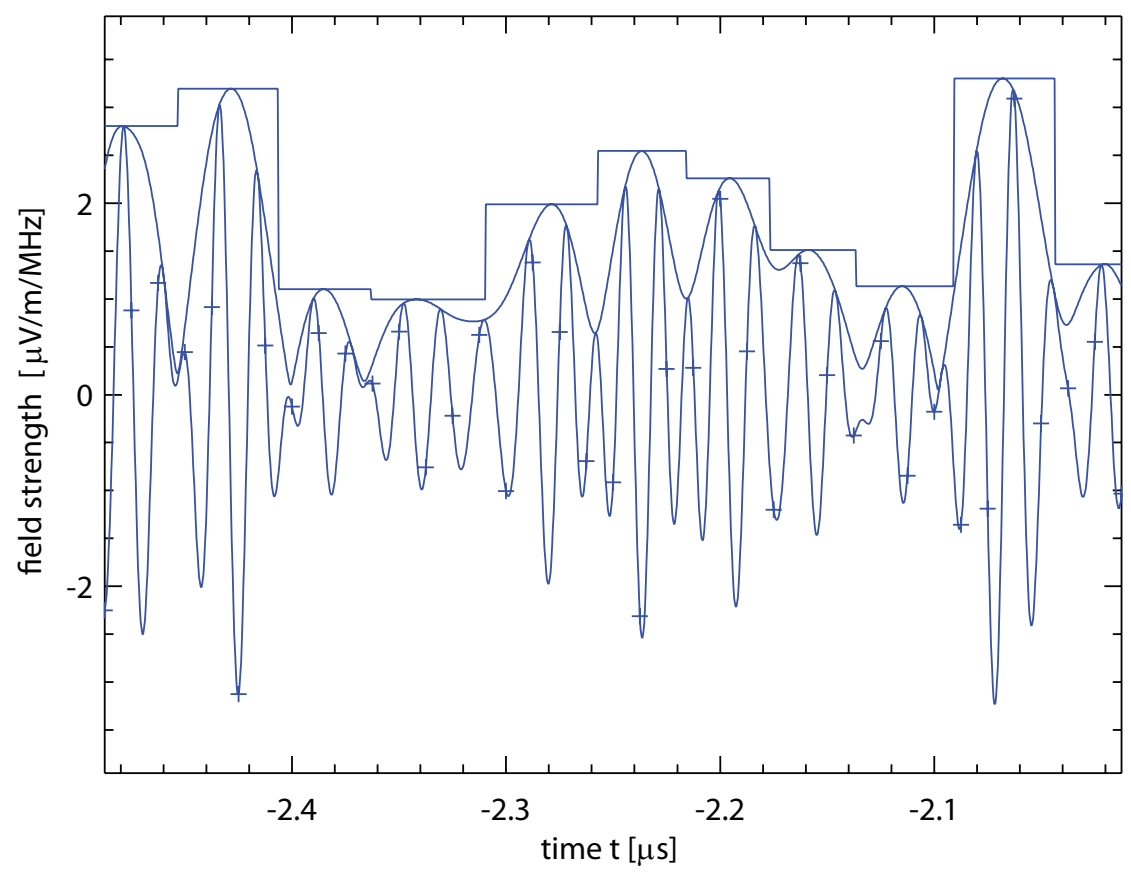

Figure 6.3.: Typical noise measured with a LOPES antenna (1/20 of a normal noise interval): sampled data points, up-sampled field strength and Hilbert envelope. The noise level is measured as the weighted average of the local maxima of the envelope. This is the average level of the plotted step function with the length of each step used as weight for averaging, and a step exactly in the middle between two local maxima of the envelope. This definition of noise is unusual, but consistent with the signal definition as local maximum of the envelope. 
Table 6.4.: Mean signal-to-noise ratios of pure noise, i.e., $A_{\text {true }}=0$, measured by different noise methods normalized to method 5 . The normalization is done per measurement, before calculating the mean and spread (standard deviation). The spread corresponds to the error made when scaling the results from another method to method 5 . The given uncertainties are the semi-difference between the results obtained with the LOPES dual polarization setup and the LOPES 3D setup. As expected, the normalized mean corresponds almost exactly to the reciprocal of the unnormalized mean signal-to-noise ratios of pure noise (table 6.3).

\begin{tabular}{cccc}
\hline Method & Normalized mean & Spread & Relative spread \\
\hline 1 & $0.477 \pm 0.007$ & $0.010 \pm 0.002$ & $(2.1 \pm 0.4) \%$ \\
2 & $0.602 \pm 0.009$ & $0.018 \pm 0.005$ & $(3.0 \pm 0.8) \%$ \\
3 & $0.734 \pm 0.010$ & $0.016 \pm 0.003$ & $(2.2 \pm 0.4) \%$ \\
4 & $0.955 \pm 0.006$ & $0.020 \pm 0.001$ & $(2.1 \pm 0.1) \%$ \\
5 & 1 & 0 & 0 \\
6 & $0.803 \pm 0.013$ & $0.051 \pm 0.016$ & $(6.3 \pm 1.8) \%$ \\
\hline
\end{tabular}

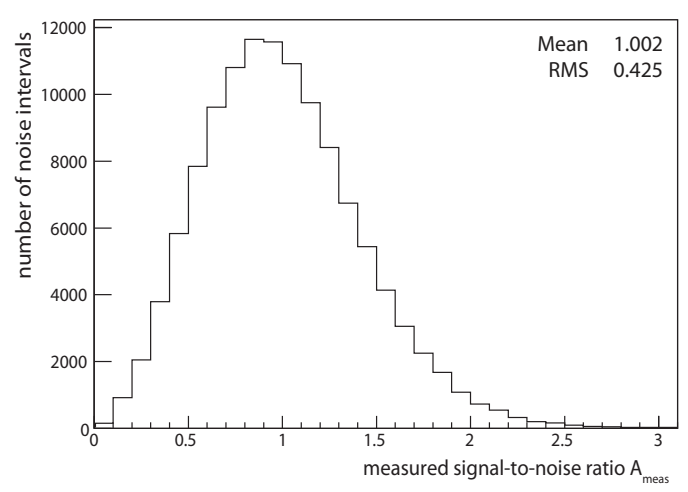

(a) histogram for method 5

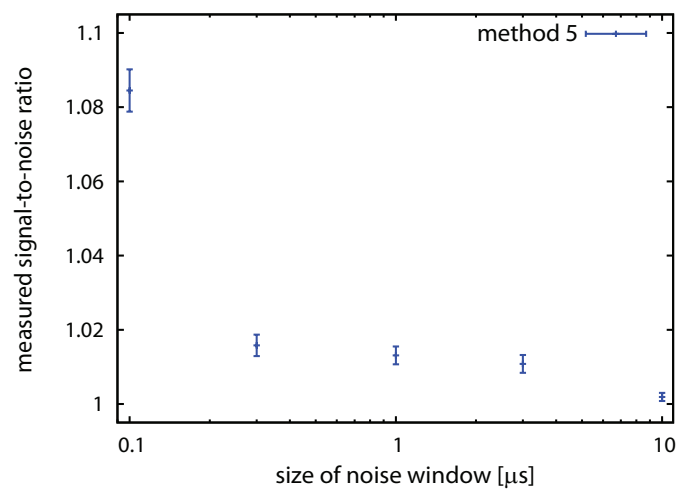

(b) dependence on noise window size

Figure 6.4.: Signal-to-noise ratios of pure noise, i.e., $A_{\text {true }}=0$ : (a) shows a histogram of the signal-to-noise ratios $A_{\text {meas }}$ measured with method 5 , for a noise window size of $10 \mu \mathrm{s}$. The distribution is neither a Gaussian, nor a Rayleigh, nor a Poisson distribution. (b) shows the dependence of the mean $A_{\text {meas }}$ on the size of the noise window. The error bars are the semidifference of the mean $A_{\text {meas }}$ obtained with the LOPES dual polarization setup and the LOPES 3D setup. 


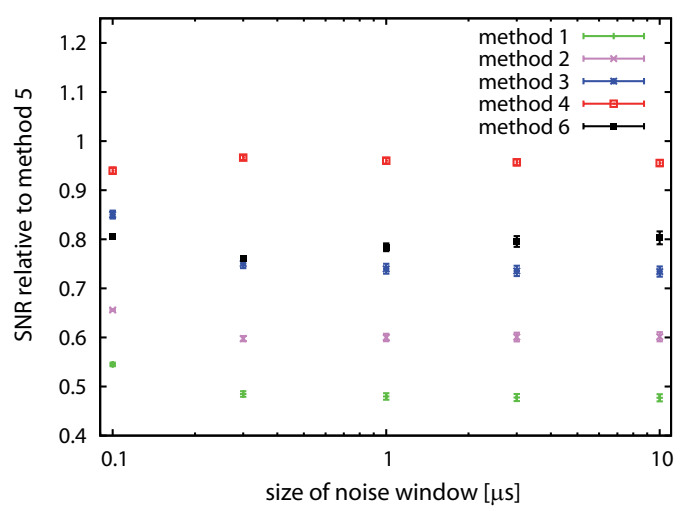

(a) different noise methods/ default method 5

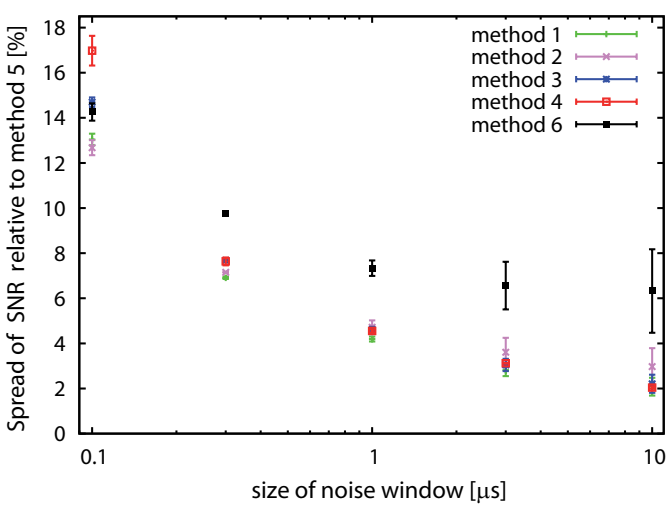

(b) relative spread

Figure 6.5.: Signal-to-noise ratios of pure noise, i.e., $A_{\text {true }}=0$, measured by different noise methods relative to method 5: (a) There is almost no dependency on the noise window size. (b) The relative spread (standard deviation divided by mean) corresponds to the error made when scaling results from one method to the default method 5 . It decreases with an increasing noise window size. The error bars are the semi-difference of the results obtained with the LOPES dual polarization setup and the LOPES 3D setup. The values for a noise window size of $10 \mu \mathrm{s}$ are given in table 6.4.

Tentative approaches to fulfill the consistency criterion have been to measure the noise level either as mean of the local maxima of the trace (method 3) or as the mean of the local maxima of a Hilbert envelope (method 4). Both methods still yield average signal-to-noise ratios of pure noise larger than 1, and do not fulfill the consistency criterion. In the case of method 4, this was surprising because it also works with the Hilbert envelope - like the signal measurement does. The reason is that higher local maxima are likely to have a larger distance to their neighbors than lower local maxima have. Hence, for a consistent measurement of noise, the local maxima have to be weighted by their distance to the neighboring maxima.

The calculation of the noise level as a weighted average of local maxima of the Hilbert envelope (method 5) is illustrated in figure 6.3. Indeed, it yields on average signal-to-noise ratios close to one for pure noise, i.e., $A_{\text {true }}=0$, and fulfills the presented consistency criterion (see figure 6.4(a)). Slight deviations from 1 are due to boundary effects at the edge of the noise window. For a window size of $10 \mu \mathrm{s}$, the deviation is in the order of a few per-mill and negligible in comparison to many other experimental uncertainties. However, figure 6.4(b) shows that these boundary effects play a significant role if the noise window is smaller than a few $\mu$ s. Table 6.3 shows that method 5 is the only tested method which is consistent with the method for signal measurement in individual LOPES antennas.

Consequently, for any further LOPES analyses at the level of single antennas, the noise level is determined with method 5, i.e., as weighted average of the local maxima of the envelope. A noise window size of $10 \mu$ s seems to be sufficient. It facilitates the transferability of the proposed method, because this noise window size of $10 \mu \mathrm{s}$ corresponds to the typical trace lengths of radio experiments like AERA. If 


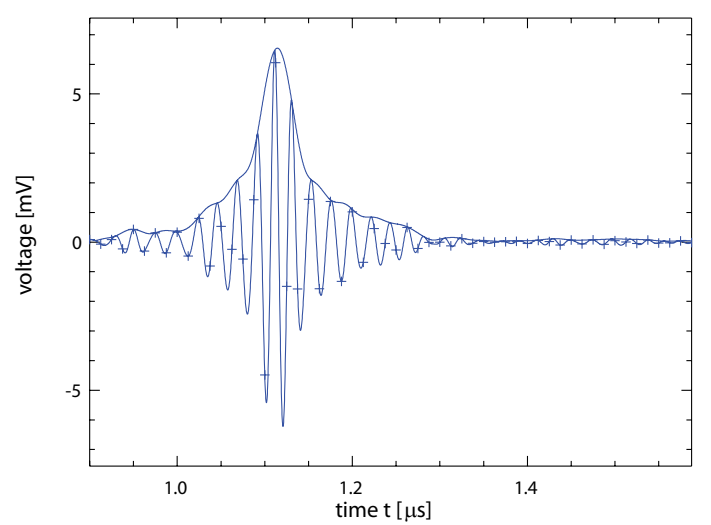

(a) pulse 1

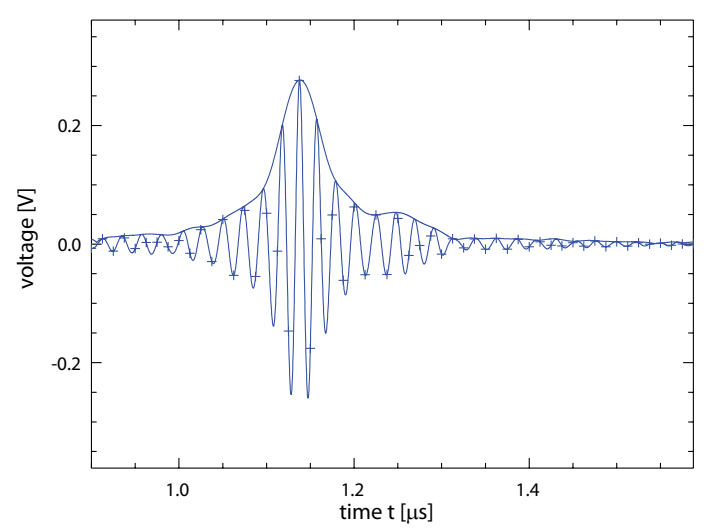

(b) pulse 3

Figure 6.6.: Four different test pulses are generated by a pulse generator to study the influence of noise on pulse amplitudes and arrival times. Test pulses 2 and 4 can be found in figure 4.7 (left and right). The original amplitudes of the generated test pulses are different (see y-axis). However this is unimportant for the present analysis, since all test pulses are scaled in software to certain true amplitudes $A_{\text {true }}$.

computing capacities are limited, the noise level can be measured by methods with a faster calculation time than method 5 , and scaled down to the proposed method 5 , accepting an error of only a few percent (see table 6.4 and figure 6.5). Moreover, the scaling factors of table 6.4 can be used to compare the results of different radio experiments and the corresponding analyses with each other.

\subsection{Influence of noise on pulse amplitudes}

For the analysis of the radio pulse at individual antennas, e.g., the reconstruction of the lateral distribution, it is important how the measured pulse amplitudes are affected by noise. The influence of noise has been investigated with test pulses. The aim of the present studies is to determine the average true signal amplitude $A_{\text {true }}$ and its error $\Delta A_{\text {true }}$ for a LOPES measurement in an individual antenna as a function of the measured signal-to-noise ratio $A_{\text {meas }}$, i.e., $A_{\text {true }}\left(A_{\text {meas }}\right)$ and $\Delta A_{\text {true }}\left(A_{\text {meas }}\right)$.

Since the true amplitude and shape of real air shower induced radio pulses is unknown, the influence of noise on real pulses cannot be investigated directly. Therefore, known test pulses emitted by a pulse generator have been recorded with LOPES (see figure 6.6), scaled in amplitude, and added in the LOPES analyses software to noise measured with LOPES. In this process, the same noise events and the same procedure as in the previous section have been used. The intrinsic noise level of the test pulse measurements is negligible. An alternative would have been to study the noise influence with simulations. However, it is not clear how well simulations can describe the pulse shape. Moreover, employing results obtained with theoretical models already for data conditioning might induce a bias when LOPES data are used to test these models. 


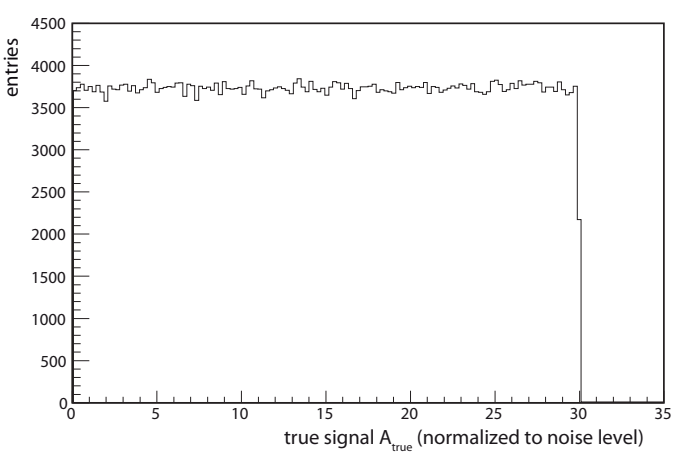

(a) flat input distribution

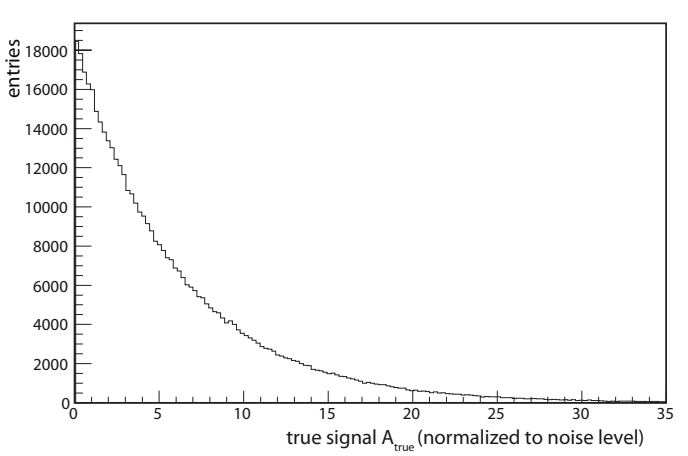

(b) exponential input distribution

Figure 6.7.: The amplitude of the test pulses was scaled to produce a flat and and exponential input distribution of $A_{\text {true }}$.

To study the influence of noise over a large range of signal-to-noise ratios, the test pulse amplitudes have been scaled to a certain true signal amplitude $A_{\text {true }}$ normalized to the noise level (i.e., $A_{\text {true }}=1$, if noise and test pulse are of equal height). A flat distribution of $A_{\text {true }} \leq 30$ has been used for scaling the test pulses before adding them to noise (see figure 6.7). To enlarge the statistics for low signal-to-noise ratios, the same test pulses and noise events have been used a second time, but with a scaling factor corresponding to $A_{\text {true }} \leq 6$. This range of low signal-to-noise ratios is extremely relevant for LOPES, because in an average event almost $80 \%$ of the LOPES antennas have signal-to-noise ratios $<4$. To check the influence of the flat $A_{\text {true }}$ input distribution, also an exponentially decaying distribution with a decay constant of 6 (in units of the noise level) has been examined. It will be shown that the differences resulting from assuming a flat distribution or an exponential distribution are smaller than other sources of uncertainties. In the LOPES standard analysis pipeline, the results obtained with the flat distribution are applied to correct for the noise influence.

The flat input distribution of $A_{\text {true }}$ has been chosen because the real distribution is unknown. The real distribution will be different for each event as it depends on shower parameters like primary energy and arrival direction. For the most accurate reconstruction of those shower parameters it might be advisable to repeat the noise analysis for each event with an input distribution of $A_{\text {true }}$ corresponding to the lateral distribution expected either from previous measurements or simulations. At the moment, the focus lies on comparing lateral distributions of data and simulation, and any presumptions could introduce a bias in this comparison. Furthermore, the precision of any LOPES reconstruction is limited by noise, the small lateral extension, and a lack of understanding of the radio emission, but probably not by details of the reconstruction procedure.

Four distinct test pulses with different shapes have been used $(10 \mathrm{~ns} \lesssim$ FWHM of envelope $\lesssim 42 \mathrm{~ns}$ ). Since 120,000 different noise intervals are available from the selected LOPES events, this yields a total statistics of 960,000 entries for the two flat distributions of $A_{\text {true }}$, and 480,000 for the exponential distribution. For each entry, the true pulse amplitude $A_{\text {true }}$ of the scaled test pulse is available, as well as 


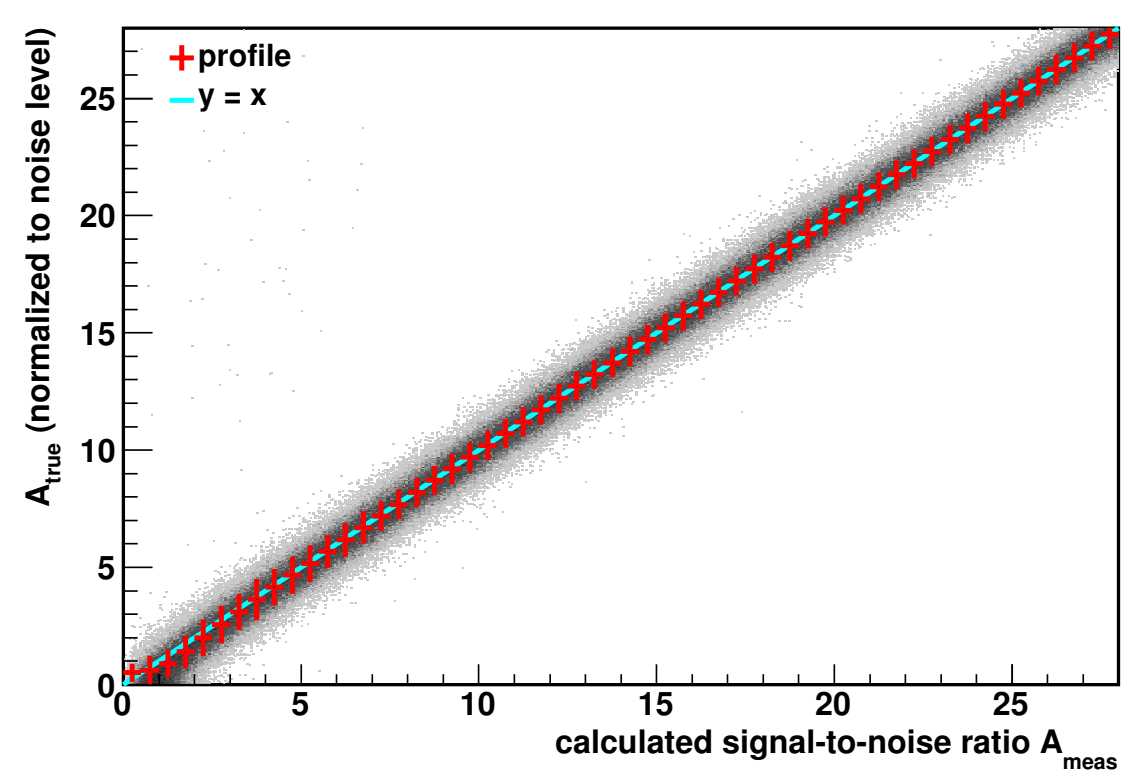

(a) large signal-to-noise ratios, for flat input distribution

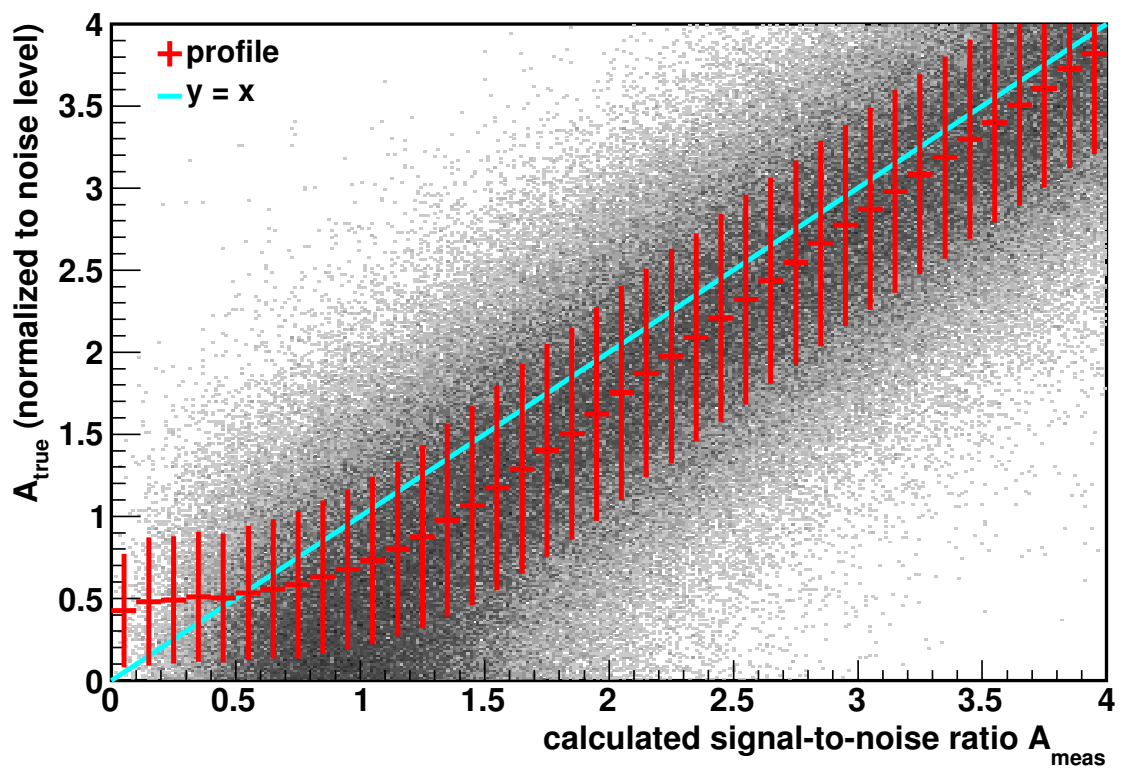

(b) sample for small signal-to-noise ratios, for flat input distribution

Figure 6.8.: True signal $A_{\text {true }}$ of the scaled test pulses over the signal-to-noise ratio $A_{\text {meas }}$, calculated after adding the scaled test pulse to measured noise. The profile of mean and standard deviation $\left(=\Delta A_{\text {true }}\right)$ is used to obtain a relation $A_{\text {true }}\left(A_{\text {meas }}\right)$ for the correction of real measurements. The region at low signal-to-noise ratios is extremely relevant for data analysis: For the selection of chapter $7,40 \%$ of the measurements at individual antennas have a signal-to-noise ratio $A_{\text {meas }}<2$, and $39 \%$ a signal-tonoise ratio $2 \leq A_{\text {meas }}<4$ (cf. figure 6.9 ). 


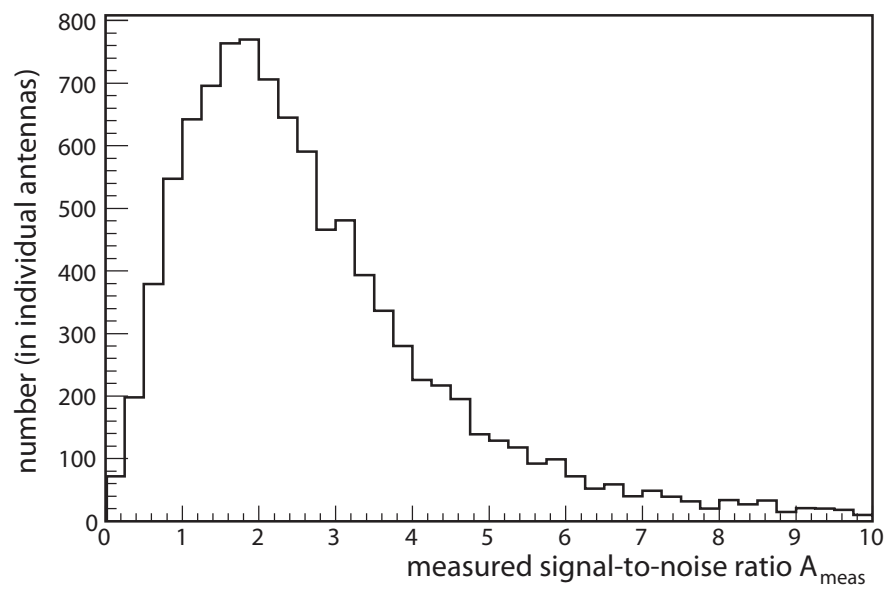

Figure 6.9.: Distribution of the measured signal-to-noise ratios in individual antennas, for the selection of chapter 7 . For each event, there is one entry for each antenna. About $3 \%$ of the measured signal-to-noise ratios are lager than 10, and not displayed in the histogram.

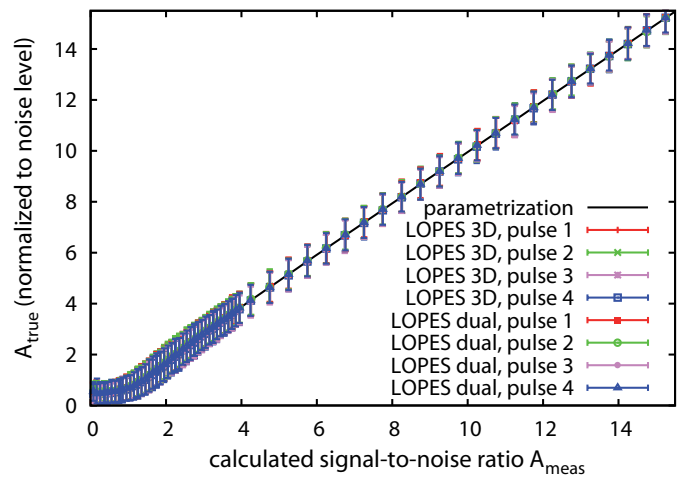

(a) pulse shape

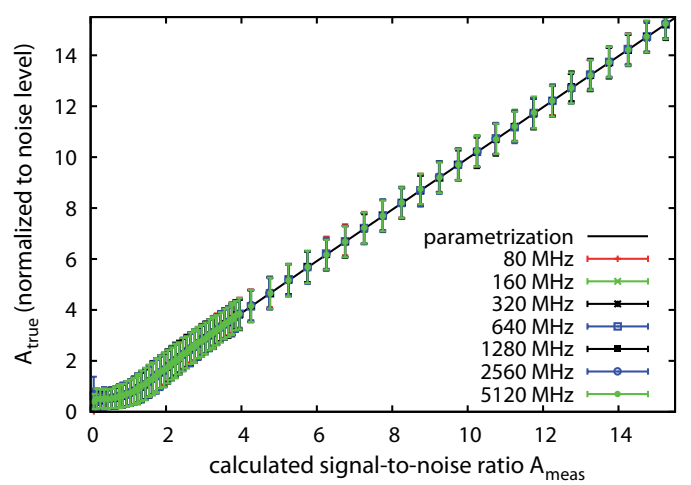

(c) up-sampling

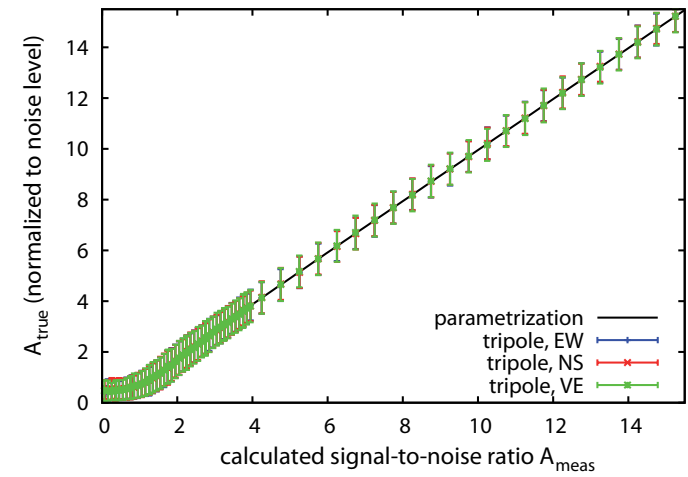

(b) polarization

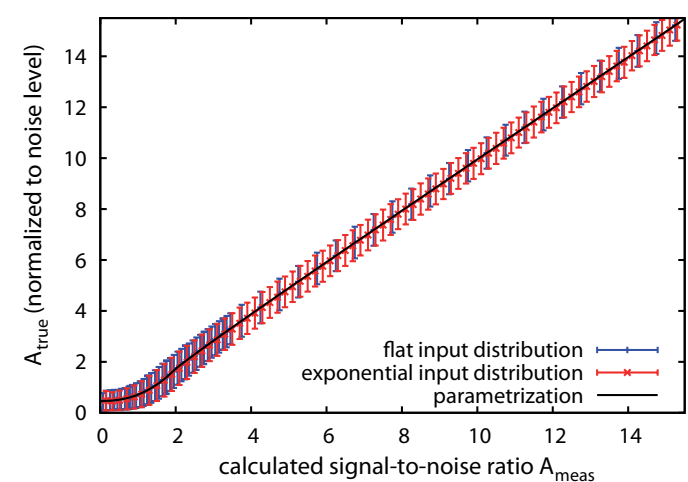

(d) probability distribution

Figure 6.10.: Check for systematic effects of the relation $A_{\text {meas }}\left(A_{\text {true }}\right)$. The plot shows profiles (mean and standard deviation, cf., figure 6.8), and the parametrization obtained with the flat input distribution. All studied systematic effects are much smaller than the standard deviation $\Delta A_{\text {true }}$. 
the signal-to-noise ratio $A_{\text {meas }}$ calculated after adding the test pulse to the noise (see figure 6.8). Although, the test pulse is added to the noise in software, the calculated signal-to-noise ratio $A_{\text {meas }}$ corresponds to the signal-to-noise ratio which would have been obtained in a real measurement of a pulse with the same shape and amplitude as the test pulse.

To appropriately account for the noise influence on measured pulse amplitudes, a relation $A_{\text {true }}\left(A_{\text {meas }}\right)$ is necessary as well as the measurement error $\Delta A_{\text {true }}\left(A_{\text {meas }}\right)$. Therefore, the mean $A_{\text {true }}$ and its standard deviation $\Delta A_{\text {true }}$ are obtained for each $A_{\text {meas }}$ with a profile (see figure 6.8). Each bin of the profile contains about 10,000 entries, except for the bins with a very low signal-to-noise ratio $A_{\text {meas }}$ close to 0 , where the statistics gets lower, until 79 entries in the first bin, i.e., $A_{\text {meas }}<0.1$. Signal-tonoise ratios close to the border of the $A_{\text {true }}$ input distribution, i.e., $A_{\text {meas }} \geq 4$ for the input distribution of $A_{\text {true }}<6$, respectively $A_{\text {meas }} \geq 28$ for the input distribution of $A_{\text {true }}<30$, have not been used for analysis. ${ }^{4}$

Various systematic effects have been checked, but no significant influence on the relation $A_{\text {true }}\left(A_{\text {meas }}\right)$ could be found (see figure 6.10 ), which facilitates accounting for noise effects in real measurements. First, the relation $A_{\text {true }}\left(A_{\text {meas }}\right)$ does not depend on the pulse shape. Thus, it should be robust against variations in the shape of real air shower pulses which is expected to change with lateral distance (cf., chapter 2, figure 2.4). Second, no dependence on the alignment and type of the antennas used for noise measurement is observed. Third, neither the sampling frequency influences the results significantly. This probably means that the sampling frequency affects noise and amplitude measurements in a similar way, such that the net effect is negligible. Fourth, there is no significant impact by the input distribution of $A_{\text {true }}$, either. Fifth, because the test pulse generator was connected directly to the analog electronics, there might be a systematic effect caused by the antenna which is not present in this analysis. However, such an effect is most likely also negligible, since correcting or not correcting the measured test pulses for the antenna gain pattern did not change the results (not shown in the plots).

To account in the LOPES standard analysis pipeline for the noise influence on pulse amplitudes, a parametrization of the relation $A_{\text {true }}\left(A_{\text {meas }}\right)$ has been searched. For high signal-to-noise ratios, the relation $A_{\text {true }}\left(A_{\text {meas }}\right)$ can be described according to the assumption that the measured power is on average the sum of the noise power and the signal power ( $\widehat{=}$ quadratic sum of the amplitudes):

$$
A_{\text {true }}=\sqrt{A_{\text {meas }}^{2}-1} \quad\left(\text { for } A_{\text {meas }} \gtrsim 2\right)
$$

\footnotetext{
${ }^{4}$ In addition, two other methods have been tested to determine $A_{\text {true }}\left(A_{\text {meas }}\right)$ and $\Delta A_{\text {true }}\left(A_{\text {meas }}\right)$, but failed: First, the inverse function of $A_{\text {meas }}\left(A_{\text {true }}\right)$, which would be available directly, is not defined for $A_{\text {meas }}<1$. But this range is important for analysis, as $12 \%$ of the measurements in individual antennas have a signal-to-noise ratio $A_{\text {meas }}<1$ (see figure 6.9). Second, using confidence intervals instead of mean and standard deviation failed. The problem is that for any confidence level $\gamma$, there exists a minimum amplitude $A_{\min }$, for which the probability that a pulse amplitude is reduced by noise to a value $A_{\text {meas }}<A_{\min }$ is smaller than $1-\gamma$. Therefore, $A_{\text {true }}\left(A_{\text {meas }}\right)$ would be undefined for $A_{\text {meas }}$ close to 0 , at least for centered confidence intervals. However, uncentered confidence intervals yield questionable results at high signal-to-noise ratios, since $A_{\text {true }}$ would systematically deviate from the mean $A_{\text {meas }}$.
} 


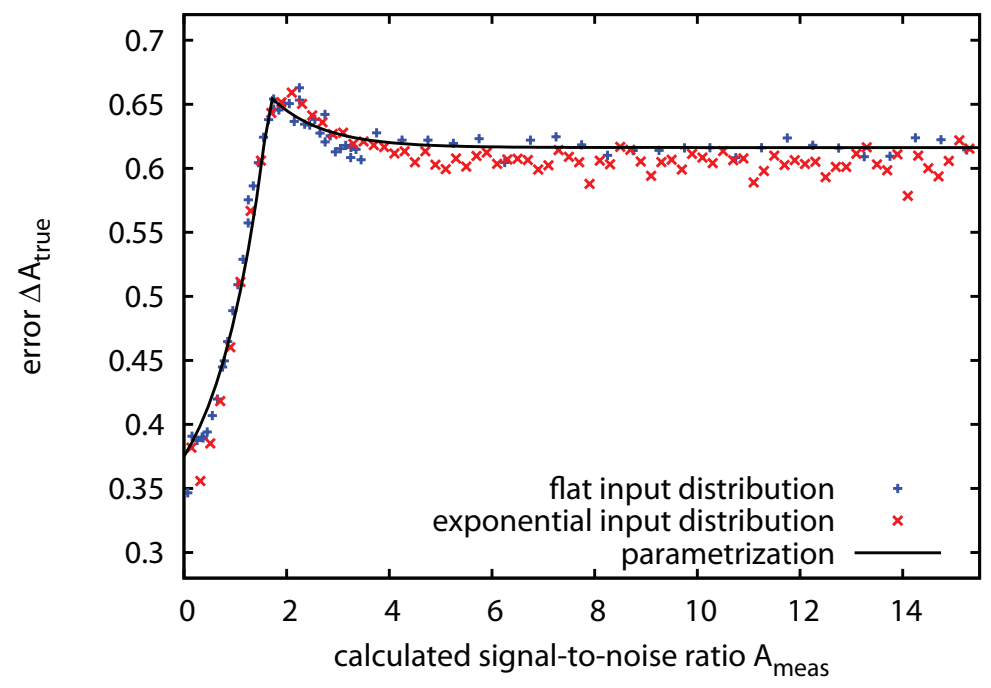

Figure 6.11.: Error $\Delta A_{\text {true }}$ (standard deviation) in dependence of the signal-to-noise ratio $A_{\text {meas }}$ calculated for scaled test pulses after adding them to noise (cf. figure 6.8), for the flat and exponential input distributions of $A_{\text {true }}$, and a parametrization obtained with the flat input distribution. The parametrization of $\Delta A_{\text {true }}$ is used for LOPES measurements in individual antennas to calculate the noise component of the measurement error.

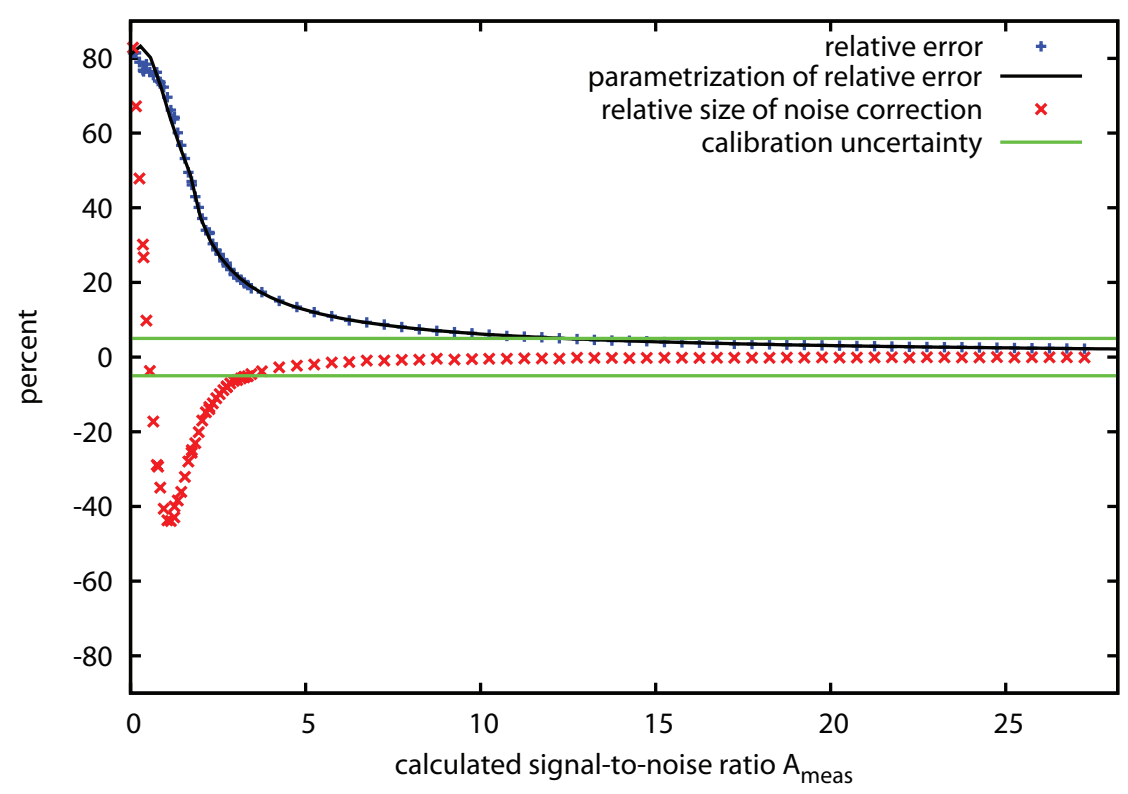

Figure 6.12.: Relative error $\Delta A_{\text {true }} / A_{\text {true }}$ (after correction for the noise influence) for a measurement at a certain signal-to-noise ratio $A_{\text {meas }}$ (blue + ), and a parametrization of the relative error. This is compared to the relative size of the noise correction $\left(A_{\text {true }}-A_{\text {meas }}\right) / A_{\text {true }}(\operatorname{red} \mathrm{x})$. As reference, also the amplitude calibration uncertainty due to environmental effects is given. 
However, this equation is not expected to hold for low signal-to-noise ratios, for the very reason that it has no solution for signal-to-noise ratios $A_{\text {meas }}<1$. Indeed, the analysis shows that it is a good approximation for data with signal-to-noise ratios $\gtrsim 2$. For lower signal-to-noise ratios, the a power law is a better description:

$$
A_{\text {true }}=a+b \cdot A_{\text {meas }}^{c} \quad \text { for } \quad A_{\text {meas }} \lesssim 2
$$

with $a=0.4628 \pm 0.0066, b=0.2491 \pm 0.0092$, and $c=2.349 \pm 0.048$, determined by a fit with a forced connection to the previous formula at $A_{\text {meas }}=2$. The combination of both formulas yield a complete parametrization of $A_{\text {true }}\left(A_{\text {meas }}\right)$, which is plotted in figure 6.10. The parametrization deviates from the test pulse data by much less than $\Delta A_{\text {true }}$, and is consequently sufficient to be used as correction function in the analysis of real data. Thus, any amplitude measurements at individual antennas are now corrected in the LOPES standard analysis pipeline by this parametrization.

Similarly, two parametrizations could be found to describe $\Delta A_{\text {true }}\left(A_{\text {meas }}\right)$, which is the standard deviation of $A_{\text {true }}$ at a fixed signal-to-noise ratio. It can be described by a rising exponential for signal-to-noise ratios $A_{\text {meas }} \lesssim 1.68$, and a falling exponential for larger signal-to-noise ratios (see figure 6.11):

$$
\begin{array}{ccc}
\Delta A_{\text {true }}=d+e \cdot \exp \left(+A_{\text {meas }}\right) & \text { for } & A_{\text {meas }} \lesssim 1.68 \\
\Delta A_{\text {true }}=f+g \cdot \exp \left(-A_{\text {meas }}\right) & \text { for } & A_{\text {meas }} \gtrsim 1.68
\end{array}
$$

with $d=0.3103 \pm 0.0096, e=0.0647 \pm 0.0029, f=0.6162 \pm 0.0010$, and $g=$ $0.213 \pm 0.018$, obtained from a fit.

In the LOPES standard analysis pipeline, the noise dependent measurement error of pulse amplitudes is calculated by this parametrization. Although it does not perfectly describe $\Delta A_{\text {true }}$ for the exponential input distribution, the deviation can be neglected, because it is smaller than the LOPES calibration uncertainty of $5 \%$.

Plotting the relative size of the error, i.e., $\Delta A_{\text {true }} / A_{\text {true }}$, illustrates that for signalto-noise ratios $\lesssim 10$, noise is the dominating error source (see figure 6.12 ). The relative size of the noise correction $A_{\text {true }}\left(A_{\text {meas }}\right)$ becomes larger than the calibration uncertainty at signal-to-noise ratios $A_{\text {meas }} \lesssim 4$. Therefore, correcting pulse amplitudes for the noise influence becomes mandatory for signal-to-noise ratios $\lesssim 4$, and therewith for the majority of LOPES events.

\subsection{Influence of noise on lateral distributions}

Since noise influences the amplitude at each individual antenna, it also affects the lateral distribution of the air shower induced radio emission. As has been shown in the previous section, noise is more likely to increase the measured amplitude than decrease it. The strongest influence is for measurements at low signal-to-noise ratios which generally occur more often at large lateral distances. Therefore, noise on average does not only increase the overall amplitude, but also flattens lateral distributions to large distances. If lateral distributions are fitted by an exponential (cf., section 7.1.3), a correction for the noise influence should lead to a lower amplitude parameter $\epsilon_{100}$, and a lower slope parameter $R_{0}$, compared to the situation without any correction for the noise influence, e.g., in previous analyses, like in [34] and [47]. 

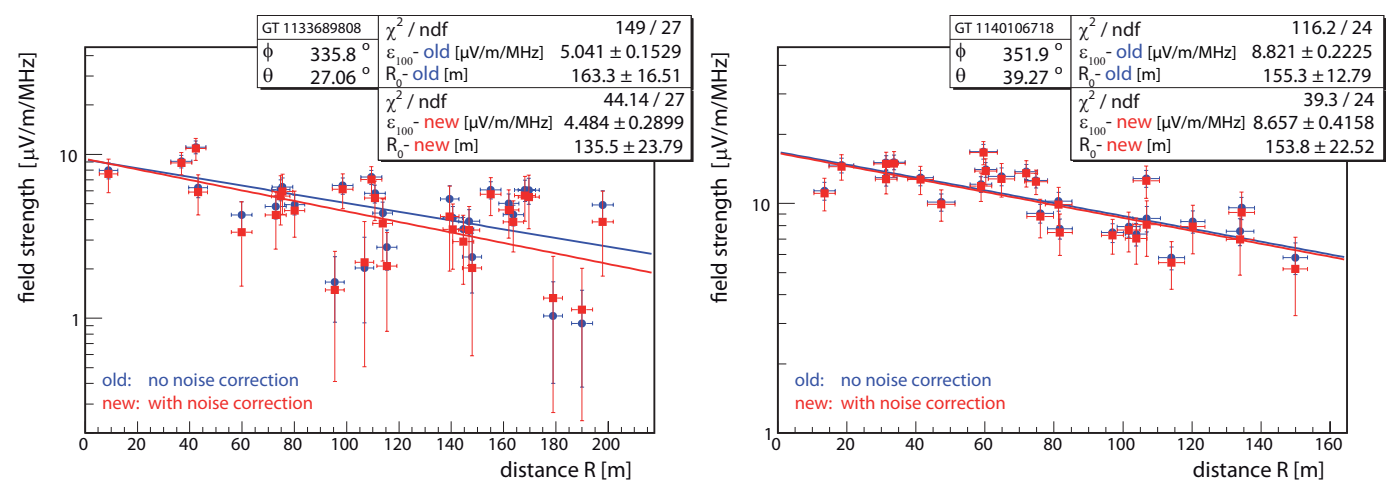

Figure 6.13.: Effect of noise on two example lateral distributions from a typical selection of LOPES events. Left: typical event. Right: event with a high signal-to-noise ratio even at the most distant antennas.

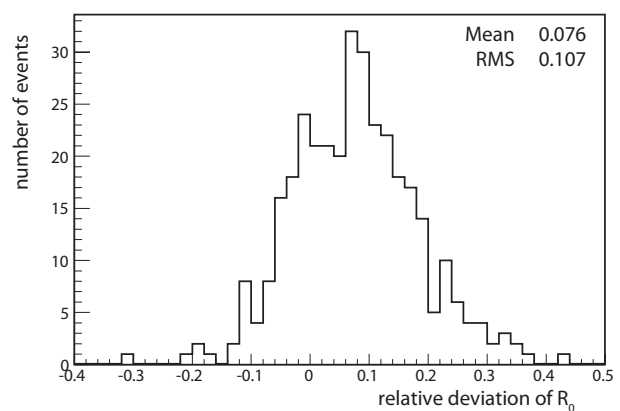

(a) slope, typical selection

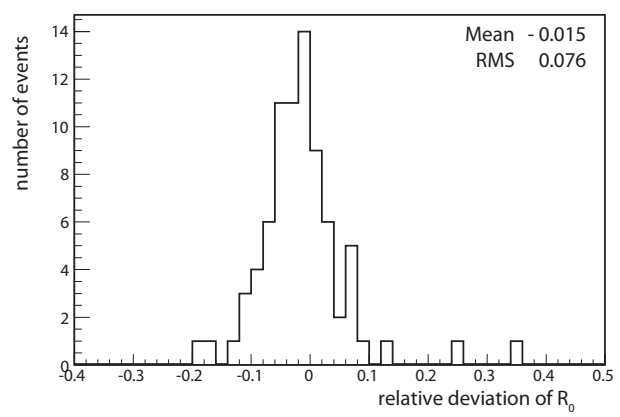

(c) slope, cut on high SNR

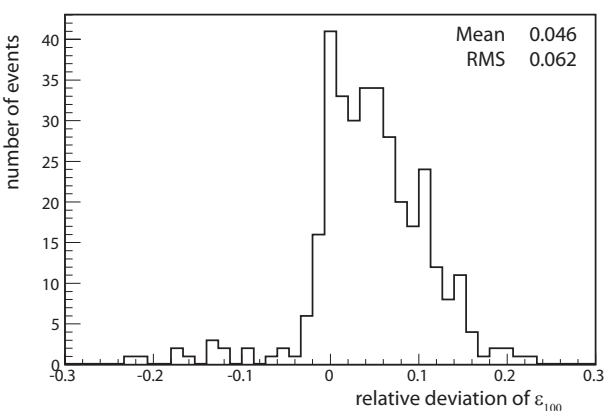

(b) amplitude, typical selection

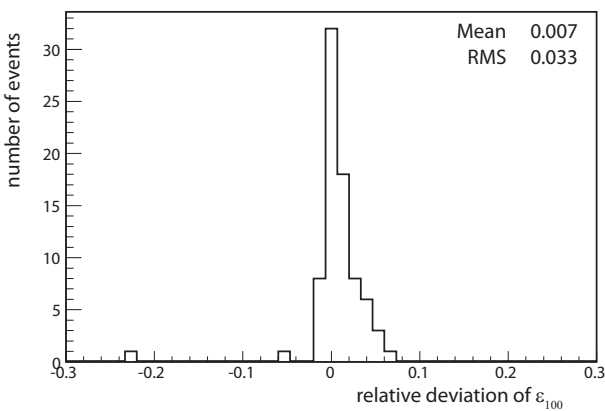

(d) amplitude, cut on high SNR

Figure 6.14.: Effect of noise on the amplitude and slope parameter $\epsilon$ and $R_{0}$ of lateral distributions. Plotted is the relative deviation with and without correction for the noise influence: (uncorrected - corrected)/uncorrected. Hence, positive values mean that the fit parameters have decreased by correcting for the noise influence. The effect becomes smaller at high signal-to-noise ratios (CC amplitude/RMS $(\mathrm{CC})>25$ ). 


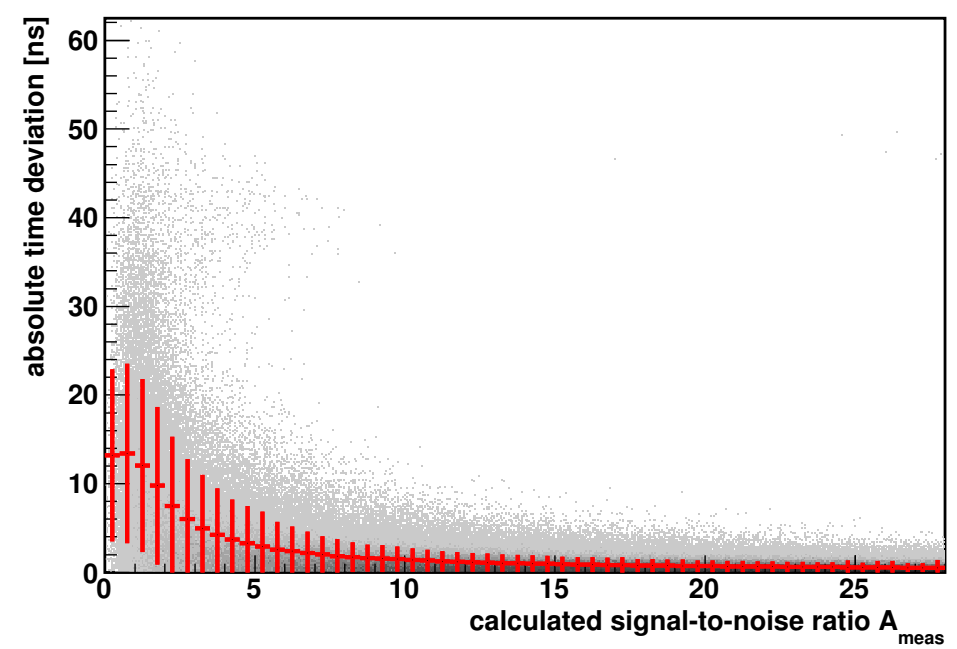

Figure 6.15.: Absolute deviation between the time of the envelope maximum before and after adding noise to the pulse. The profile displays mean and standard deviation, where the mean is interpreted as the time uncertainty $\Delta t$ of a measurement at the corresponding signal-to-noise ratio $A_{\text {meas }}$.

To check how large the effect of noise on lateral distributions is, all events from the selection of chapter 7 have been processed with and without applying the noise correction on measured amplitudes (see previous section). Figure 6.13 shows two example events. The noise influence is negligible if all antennas contributing to the measured lateral distribution have a high signal-to-noise ratio. This can also be seen in histograms comparing the noise influence for all events with events at a very high signal-to-noise ratio (figure 6.14). The net effect on $\epsilon_{100}$ and $R_{0}$ for events with a typical signal-to-noise ratio is $\sim 5 \%$, which is smaller than the typical uncertainties of $\epsilon_{100}$ and $R_{0}(\sim 20 \%)$. However, the noise effect is not a simple uncertainty, but a systematic increase of $\epsilon_{100}$ and $R_{0}$. Consequently, a correct treatment of noise is mandatory for analyses with lateral distributions. Otherwise, a systematic bias on the slope and height of lateral distributions is introduced.

In particular, an appropriate treatment of noise becomes crucial, if the composition of the primary cosmic ray particles is reconstructed via the slope of lateral distributions (cf., section 7.4). Since noise flattens only lateral distributions at low signal-to-noise ratios, i.e., at energies close to the detection threshold, it could mimic a change in slope with energy. This could easily be misinterpreted as a change in composition with energy, or disguise a real change in composition.

\subsection{Influence of noise on pulse arrival time measurements}

The pulse arrival time $t$ in an antenna is measured with the maximum of a Hilbert envelope of the up-sampled field strength. The maximum closest to the time expected from the cross-correlation beamforming is taken, i.e., the same maximum as for the determination of the pulse amplitude (cf., chapter 8). Measuring the pulse time 
at the maximum is necessary because the signal-to-noise ratio usually is very low (see figure 6.9), and the maximum can be best distinguished from noise. For time calibration, different methods for pulse time measurements have been investigated, but none turned out to be more precise than determining the maximum time (see also chapter 8 , section 8.2.1).

The influence of noise on the measurement of pulse arrival times has been studied in a similar way as the influence on pulse amplitudes. The same test pulses have been added to the same noise intervals, and the absolute deviation of the maximum time $t$ due to noise has been determined (see figure 6.15). It decreases with increasing signalto-noise ratio. The mean absolute deviation $\Delta t$ at a signal-to-noise ratio $A_{\text {meas }}$ has been obtained with a profile. By definition, $\Delta t$ is always positive. $\Delta t$ is interpreted as the statistical, noise induced uncertainty of pulse arrival time measurements.

Several systematic influences have been checked (see figure 6.16). First, $\Delta t$ depends on the pulse shape, but no correlation with easily accessible parameters like pulse amplitude or FWHM could be found. For this reason, and as it is unknown which test pulse shape does best describe the real cosmic ray induced pulses, the LOPES standard analysis pipeline takes into account only the mean result of all pulse shapes. Second, no dependence on the polarization and antenna type was found. Third, as expected, $\Delta t$ depends on the sampling rate. The systematic uncertainty due to the sampling rate becomes negligible against the time calibration uncertainty $(\sim 2 \mathrm{~ns}$, cf. 4.3), if an up-sampling factor of at least 8 is chosen, i.e., a sampling frequency of at least $640 \mathrm{MHz}$. Fourth, like for the pulse amplitudes, the input distribution of $A_{\text {true }}$ has almost no influence on $\Delta t$, neither. Fifth, in principle, an asymmetric pulse can also be shifted on average, leading to an offset of the pulse arrival time. This effect is neglected, since no significant offset could be observed in this analysis. Sixth, the effect of the bandwidth on $\Delta t$ or its dependency on the pulse shape could not be studied, as the effective bandwidth is fixed to $43-74 \mathrm{MHz}$ by LOPES. Hence, $\Delta t$ and its systematic dependencies might be quantitatively different at experiments with a different bandwidth, like AERA $(30-80 \mathrm{MHz})$.

An empirical parametrization could be found for the dependency of the pulse arrival time uncertainties $\Delta t$ on the signal-to-noise ratio $A_{\text {meas. }}$ Although this parametrization has no deeper physical motivation, it provides an easy way to implement the uncertainty in the standard analysis pipeline, avoiding tables and interpolations.

$$
\begin{gathered}
\Delta t=\left(16.6-\left(2 A_{\text {meas }}-1.3\right)^{2}\right) \mathrm{ns} \quad \text { for } \quad A_{\text {meas }} \lesssim 1.84 \\
\Delta t=20.5 \cdot A_{\text {meas }}^{-1.03} \mathrm{~ns} \quad \text { for } \quad A_{\text {meas }} \gtrsim 1.84
\end{gathered}
$$

All coefficients have been adjusted by hand to fit the data. The antiproportionality of $\Delta t$ and $A_{\text {meas }}$ is in agreement with experience from radio astronomy.

Summarizing, not only for measurements of pulse amplitudes but also for arrival times, noise is the dominating source of the total uncertainty for signal-to-noise ratios $A_{\text {meas }} \lesssim 10$. Because in many LOPES events the cosmic ray radio pulse has a low signal-to-noise ratio in most of the antennas, an appropriate treatment of noise is absolutely necessary for the correct reconstruction of the lateral distributions of pulse amplitudes and arrival times (cf., chapters 7 and 8). Therefore, the parametrizations of the noise influence have been included in the LOPES analysis software. 


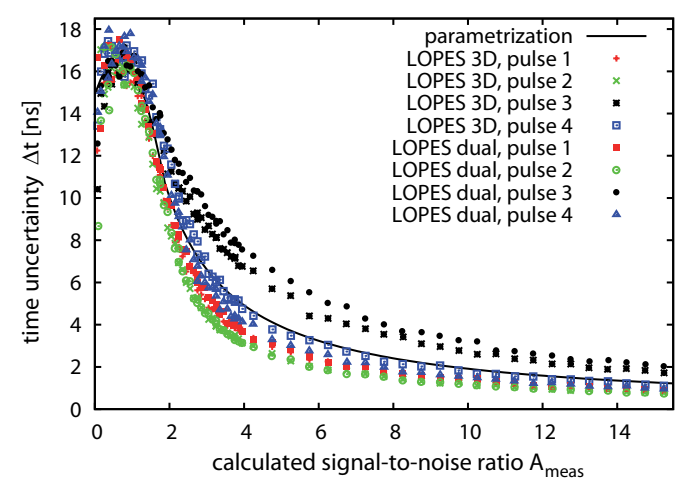

(a) pulse shape

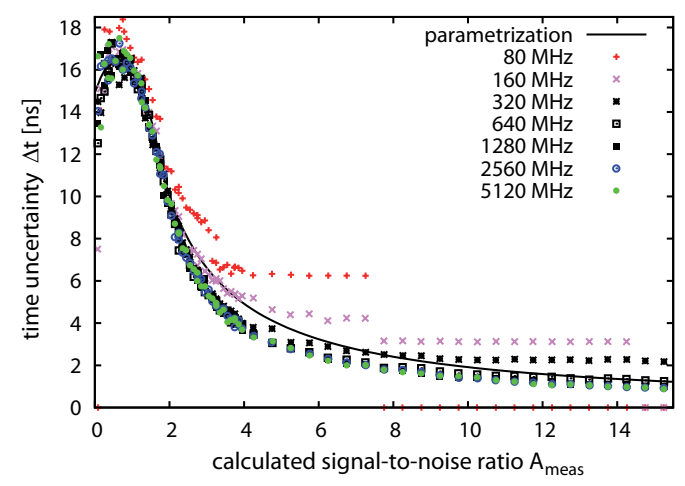

(c) up-sampling

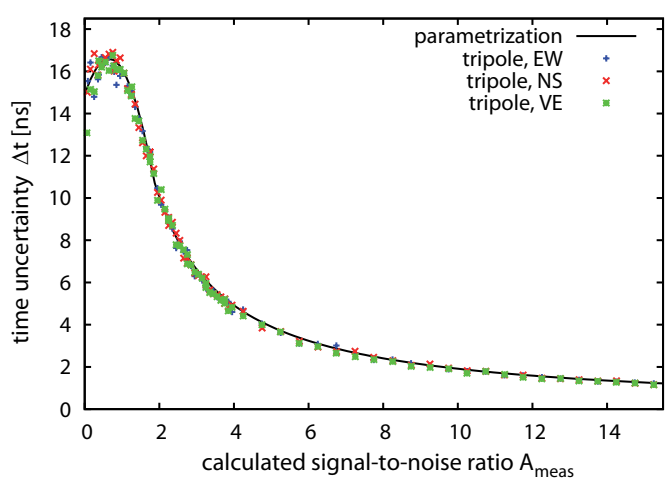

(b) polarization

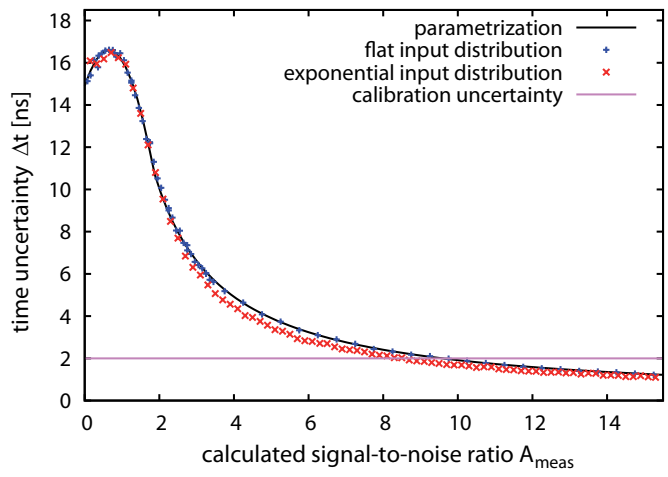

(d) input distribution

Figure 6.16.: Check for systematic effects: the pulse time uncertainty $\Delta t$ due to noise depends on the pulse shape in a non-trivial way. It also depends on the sampling frequency, but does not depend on the polarization and antenna type. The dependency on the input distribution of $A_{\text {true }}$ is small compared to both $\Delta t$, as well as the time calibration uncertainty of $\sim 2 \mathrm{~ns}$. As reference, the parametrization of $\Delta t$ obtained with the flat input distribution is displayed in all plots.

After the parametrizations found for LOPES have been checked with AERA, they will also be implemented in the Offline software package. Although, the general behavior of the noise influence is expected to be the same at AERA, the details, i.e., the coefficients of the parametrizations, might differ. Slight differences could originate for instance from the extended frequency band of AERA $(30-80 \mathrm{MHz})$, the different pulse search procedure independent of an interferometric analysis, or the fact that Offline reconstructs the vector of the electric field and does not determine the pulse amplitude in just one polarization channel. Nevertheless, the basic properties of the measurement method are the same as at LOPES: Also at AERA the signal is determined as the maximum instantaneous amplitude of a bandwidth limited measurement. In principle, the presented method for noise treatment can be transferred to any experiment based on radio or acoustic arrays, where the signal consists of a short, bandwidth limited pulse, and noise can interfere in both ways, destructively and constructively with the signal. 


\section{Lateral Distribution}

The lateral distribution of the radio signal emitted by air showers is of great interest for several reasons: it allows to test theoretical models for the emission process, and it is sensitive to the energy and mass of the primary cosmic ray particle. Moreover, the lateral distribution is important for the spacing of future antenna arrays. In a previous work it was already shown [47] that REAS2 simulations which are based on an incomplete implementation of the geosynchrotron model, cannot exhaustively reproduce lateral distributions measured by LOPES. The present study shows that lateral distributions predicted by REAS3 are compatible with LOPES data. This demonstrates the progress in the theoretical understanding of the radio emission and in the reconstruction of the lateral distribution from measured signals.

The slope of REAS3 lateral distributions differs by $\sim 20 \%$ between proton and iron showers. Thus, comparing measured per-event lateral distributions with simulations is not only useful to test models, but also yields a way to estimate the primary mass. The difference between proton and iron lateral distributions of $\sim 20 \%$ sets a scale up to which any systematic effects must be known and under control. While influences of shower parameters on the lateral distribution can probably be covered by perevent simulations, this is not true for experimental effects. Thus, any systematic uncertainties and biases in the measurement of lateral distributions must be well investigated. Moreover, understanding these effects is crucial to reproduce results, and to compare them to other experiments. A lack of quantitative reproducibility has been one of the major problems of historic experiments [50].

This chapter starts with an explanation how lateral distributions have been measured with LOPES and which events have been used. Then, the focus is on systematic effects on the amplitude and slope parameter of an exponential fit to the lateral distribution. Finally, lateral distributions measured with LOPES are compared to REAS3 simulations for proton and iron showers.

\subsection{Analysis procedure}

The lateral distribution $\epsilon(R)$ is the dependence of the radio signal amplitude $\epsilon$ on the lateral distance to the air shower axis $R$. Hence, analyzing the lateral distribution of a radio event requires the determination of the pulse amplitude $\epsilon$ at each antenna position and its lateral distance $R$ to the shower axis. Finally an exponential function is fitted to the measured amplitude at each antenna. The procedures for all tasks (determination of $\epsilon$ and $R$ and the exponential fit) are explained in this section. They are similar to the ones described in [47], but have been improved in several aspects. 


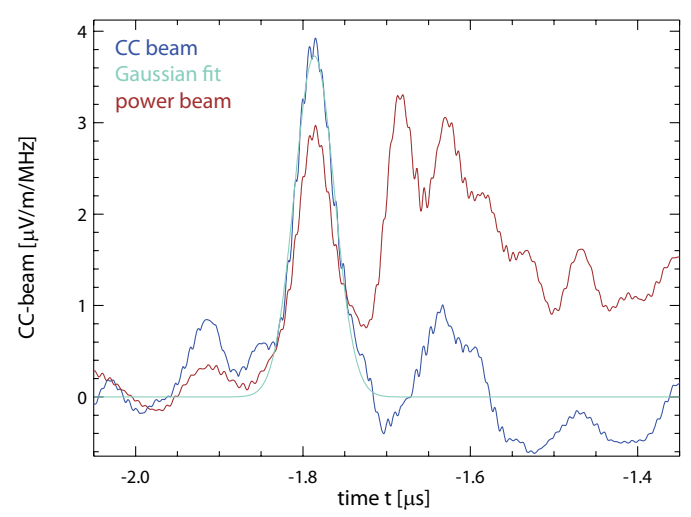

(a) smoothed cross-correlation (CC) beam

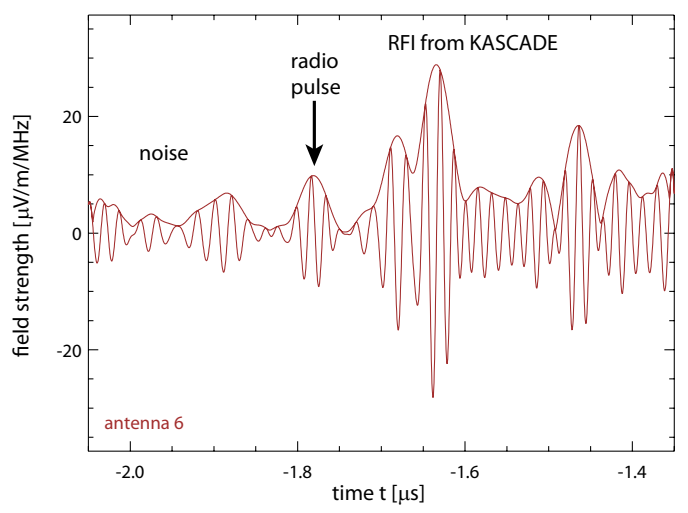

(b) antenna 6

Figure 7.1.: Example for pulse amplitude measurement: CC beam of the event shown in figure 6.2 and up-sampled electrical field strength with Hilbert envelope of antenna 6 . The time of the $\mathrm{CC}$ maximum obtained by a Gaussian fit is $-1.786 \mu \mathrm{s}$. The amplitude $\epsilon$ used for the lateral distribution is the closest maximum of the envelope: it is $9.88 \mu \mathrm{V} / \mathrm{m} / \mathrm{MHz}$ at $-1.781 \mu \mathrm{s}$. The noise level is $3.17 \mu \mathrm{V} / \mathrm{m} / \mathrm{MHz}$. It is calculated in a $10 \mu \mathrm{s}$ window ending $0.5 \mu$ s before the CC maximum, which is before the plotted range.

\subsubsection{Determination of the amplitude}

Due to the high noise level at the LOPES site, the integrated power is not a good measure for the strength of the air shower induced radio pulse (cf., chapter 6). Instead, the maximum instantaneous amplitude $\epsilon$ is used to quantify the radio pulse in an individual antenna. This is measured as the maximum of a Hilbert envelope of the up-sampled electrical field strength (see appendix B) with a default up-sampling frequency of $1280 \mathrm{MHz}$.

Unfortunately, not only the air shower itself, but also the KASCADE scintillators emit a radio pulse (RFI). However, this RFI from the scintillators can be distinguished from the cosmic ray induced pulse: In contrast to the air shower pulse, RFI is uncorrelated in all antennas, which results in a low amplitude of the crosscorrelation beam. Furthermore, the KASCADE RFI arrives significantly after the radio pulse at the antennas (see figure 7.1). Therefore, the maximum amplitude $\epsilon$ in each individual antenna is determined as the local maximum of the envelope closest to the cross-correlation maximum, which itself is determined with a Gaussian fit to the smoothed cross-correlation beam. The need to search for the pulse maximum only at the time precisely known from the cross-correlation beam becomes clear in the example of figure 7.1 , especially because about $2 / 3$ of the LOPES measurements in individual antennas have even smaller signal-to-noise ratios than the shown example.

In reference [47], not the local maximum but the global maximum in a certain window around the cross-correlation maximum has been used. However, this has the risk of misidentifying the radio pulse: If the window is too large, the KASCADE RFI could leak into the window, but if the window is too small, the pulse maximum could be outside of the window, since the maximum in an individual antenna can 
Table 7.1.: Definition of shower coordinates used for LOPES, and its errors

$x_{s}=-x \cdot \sin \phi+y \cdot \cos \phi$

$y_{s}=x \cdot \cos \phi \cos \theta+y \cdot \sin \phi \cos \theta-z \cdot \sin \theta$

$z_{s}=x \cdot \cos \phi \sin \theta+y \cdot \sin \phi \sin \theta+z \cdot \cos \theta$

$\Delta x_{s}^{2}=\Delta d_{\text {core }}{ }^{2}+(x \cos \phi+y \sin \phi)^{2} \Delta \phi^{2}$

$\Delta y_{s}{ }^{2}=\left(\Delta d_{\text {core }} \cos \theta\right)^{2}+(-x \sin \phi+y \cos \phi)^{2}(\cos \theta \Delta \phi)^{2}+(x \cos \phi+y \sin \phi)^{2}(\sin \theta \Delta \theta)^{2}$

$\Delta z_{s}{ }^{2}=\left(\Delta d_{\text {core }} \sin \theta\right)^{2}+(-x \sin \phi+y \cos \phi)^{2}(\sin \theta \Delta \phi)^{2}+(x \cos \phi+y \sin \phi)^{2}(\cos \theta \Delta \theta)^{2}$

with:

azimuth $\phi$ (a shower from North has $\phi=0^{\circ}$ and a shower from East $\phi=90^{\circ}$ )

zenith $\theta\left(=90^{\circ}-\right.$ elevation $)$

northing $x$, easting $y$, and height $z$

core uncertainty $\Delta d_{\text {core }}=\Delta x=\Delta y$

be shifted by noise to a time which differs from the time of the cross-correlation maximum (by $\lesssim 20 \mathrm{~ns}$, cf. section 6.5 ). For this reason, the window procedure of [47] was replaced by the search of the local maximum closest to the cross-correlation maximum. However, this requires strict quality cuts on the cross-correlation beam (see section 7.2).

Another difference to reference [47] is the implementation of the improved noise treatment for the determination of the pulse amplitude $\epsilon$ and its error $\Delta \epsilon(A$ in chapter 6 correspond to $\epsilon$ normalized to the noise level). $\Delta \epsilon$ is calculated as the squared sum of the error due to noise (see section 6.3) and the calibration uncertainty of $5 \%$ (see section 4.1). Uncertainties of the amplitude measurement which are related to the absolute scale of the field strength, are not included in $\Delta \epsilon$. They are important for the comparison of LOPES amplitudes with other experiments and simulations, but should not influence the weight of data points when fitting a function to the lateral distribution.

\subsubsection{Determination of the lateral distance}

The lateral distance $R$ of an antenna is defined as the distance from the antenna to the air shower axis. In other words, this is the distance of the shower core to the antenna position projected on the shower plane, whereby the shower plane is perpendicular to the shower axis and contains the core. Because the core position is defined as the center of the shower coordinate system, the calculation of lateral distances is easiest in shower coordinates (cf., table 7.1):

$$
R=\sqrt{x_{s}^{2}+y_{s}^{2}}
$$

whereby $x_{s}$ and $y_{s}$ describe the antenna position in shower coordinates.

The error of $R$ can be estimated by Gaussian error propagation if the errors of $x_{s}$ and $y_{s}$ are known:

$$
\Delta R=\frac{1}{R} \sqrt{\left(x_{s} \cdot \Delta x_{s}\right)^{2}+\left(y_{s} \cdot \Delta y_{s}\right)^{2}}
$$

The errors of the antenna position in shower coordinates $\left(\Delta x_{s}, \Delta y_{s}, \Delta z_{s}\right)$ depend on the geometrical errors of the KASCADE-Grande reconstruction, i.e., the error of the azimuth $\Delta \phi$, the error of the zenith $\Delta \theta$ and the error of the core position 


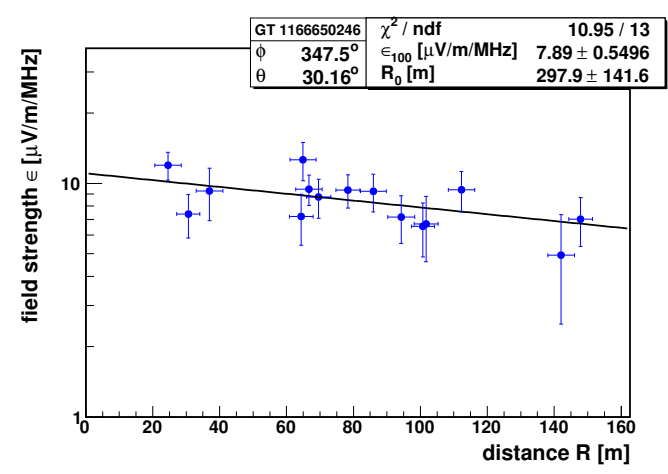

(a) KASCADE event, LOPES Dual

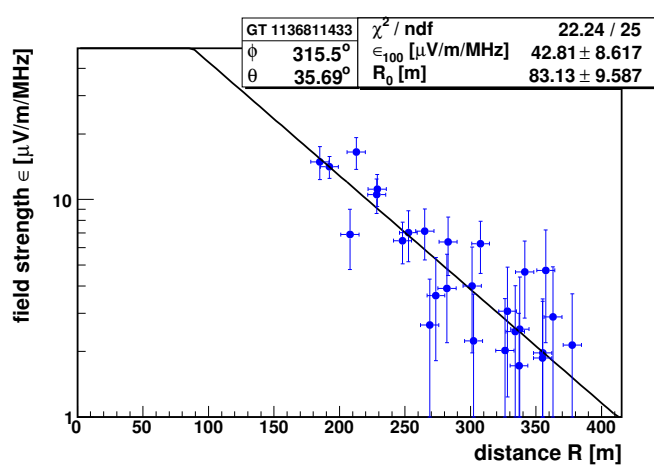

(b) Grande event, LOPES 30

Figure 7.2.: Two examples of lateral distributions measured with east-west aligned antennas of different LOPES setups and fitted with an exponential (see appendix D for further examples). The error bars have been calculated individually for each data point. The error in $R$ is predominantly determined by the core uncertainty, and the error in $\epsilon$ by noise and the calibration uncertainty.

$\Delta d_{\text {core }}$. These errors have been estimated in the energy range most interesting for LOPES $\left(10^{17} \mathrm{eV}\right.$ to $\left.10^{18} \mathrm{eV}\right)$ by comparing the true and reconstructed parameters of CORSIKA showers. The direction uncertainty is typically $<0.5^{\circ}$, and $\Delta d_{\text {core }}$ is typically $4 \mathrm{~m}$ for core positions in the KASCADE array, and $7 \mathrm{~m}$ for core positions in the Grande extension.

Furthermore, there is an uncertainty originating from the conversion of the LOPES to the KASCADE coordinate system because the antenna positions are given in LOPES coordinates and the core position in KASCADE coordinates. However, these conversion uncertainties are more than ten times lower than the reconstruction uncertainties, and are neglected in this analysis (cf., section 4.3).

The errors $\Delta x_{s}, \Delta y_{s}$ and $\Delta z_{s}$ are estimated with Gaussian error propagation under the assumption that the error of the core position $\Delta d_{\text {core }}$ is equal to the error in the $x$ and $y$ position of the core, ignoring possible correlations: $\Delta x=\Delta y=\Delta d_{\text {core }}$. One could argue that a factor of $\sqrt{2}$ should enter, but it was omitted to ensure that for vertical events the total uncertainty of the lateral distance equals the core uncertainty.

Independent of the shower inclination, the error of the lateral distance $\Delta R$ typically is in the same order as the core uncertainty $\Delta d_{\text {core }}$ of $\sim 4-7 \mathrm{~m}$. In addition, the error $\Delta z_{s}$ plays a role when determining the curvature of the radio wavefront with lateral distributions of pulse arrival times (see chapter 8).

\subsubsection{Exponential fit}

Knowing the measured signal amplitude $\epsilon_{d}$, the lateral distance $R$, and the corresponding errors, a minimizing $\chi^{2}$ fit is performed for the lateral distribution of each event (see figure 7.2 for two examples). The fitted function describes a uniform exponential decrease, like it is expected from historic experiments [50], and like it has 

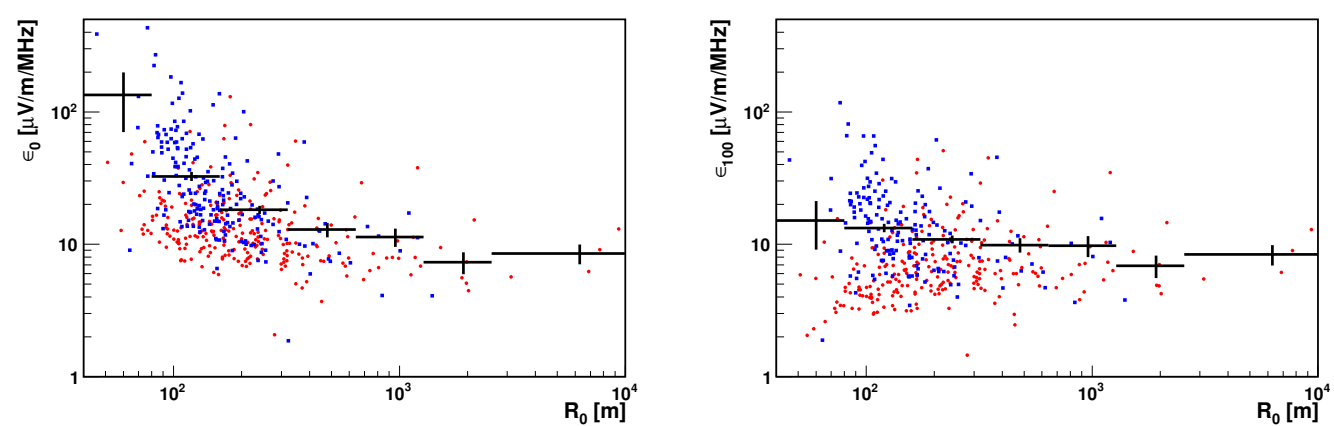

Figure 7.3.: Correlation between the two fit parameters $R_{0}$ and $\epsilon_{0}$ (left), respectively $\epsilon_{100}$ (right): red circles $=$ KASCADE events, blue squares $=$ Grande events, black $=$ profile of all events.

recently been used in [77]:

$$
\epsilon(R)=\epsilon_{d} \cdot \exp -\frac{R-d}{R_{0}}
$$

The two fitted variables are $\epsilon_{d}$, the amplitude at the lateral distance $d$, and $R_{0}$, a slope parameter. A distance $d=0 \mathrm{~m}$ was used for the fit in previous analyses $[77,85]$, but as shown in [85], the two fit variables $\epsilon_{0}$ and $R_{0}$ are correlated with each other at $d=0 \mathrm{~m}$. In this thesis, $d=100 \mathrm{~m}$ has been chosen, because this corresponds roughly to the mean lateral distance of a typical LOPES event. As can be seen in figure 7.3, this choice reduces the correlation between the two fit parameters. The remaining correlation can have various origins: no fine tuning of the distance $d$ has been performed, and the typical lateral distance is systematically different for events with core in the KASCADE array or the Grande extension. A correlation is also expected if the true, physical lateral distribution is not an exponential function.

A physical reason for a nonuniform lateral distribution function can be an azimuthal asymmetry, which is predicted by different simulations due to the variation of the net charge excess in the air shower $[123,124]$. Indeed, there are indications that the lateral distribution can only approximately be described by a uniform exponential function. Inconsistencies with the assumption of a uniform exponential function are observed on the level of individual events, corresponding to small intervals of lateral distances $(\sim 100 \mathrm{~m})$, as well as on average of all events, corresponding to a range of lateral distances $R \lesssim 300 \mathrm{~m}$.

The inconsistency on the level of individual events results in a mean reduced $\chi^{2}$ significantly larger than 1 (figure 7.4, left). The reason can be either a physical effect, like an azimuthal asymmetry of the lateral distribution, or a systematic underestimation of the error $\Delta \epsilon$. A significant underestimation of the error is unlikely, as the dominating error source is the noise present at LOPES, whose influence has been investigated with great care in chapter 6 . However, any possible correlations of $\Delta \epsilon$ in the different antennas contributing to an event have not been studied, and might cause either an underestimation or an overestimation of $\Delta \epsilon$. Moreover the high average $\chi^{2}$ can also be explained by other physical effects causing a fluctuation 

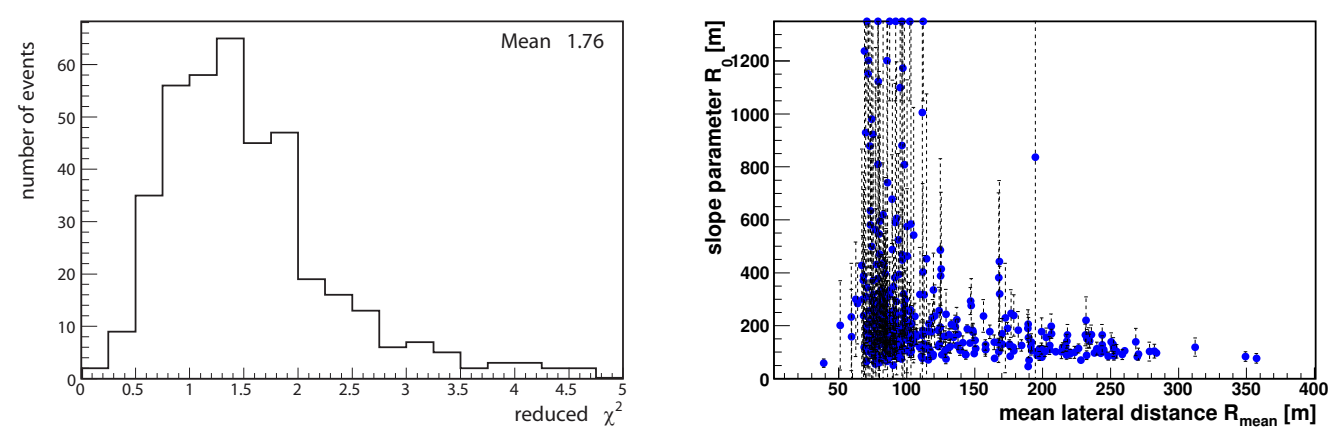

Figure 7.4.: Reduced $\chi^{2}$ (left), and relation between the fitted slope parameter $R_{0}$ and the mean lateral distance $R_{\text {mean }}$ (right), for the selection presented in section 7.2 .

of the amplitude at a certain distance. For example, the Nançay Decametric Array has observed an inhomogeneity of the radio amplitude at small scales $<100 \mathrm{~m}$ [125]. The reason is yet unknown. Maybe density fluctuations in the air shower could cause significant fluctuations in the radio amplitude at a fixed lateral distance.

Despite these problems, other functions for fitting the lateral distribution have not been tested in this thesis. Already in [34], a power law could be excluded because it overestimates the amplitude close to the shower core. A better understanding and functional description of the lateral distribution is currently under investigation in [126], too. Probably, the true lateral distribution can only be described accurately by a two dimensional function with several parameters. As LOPES can measure only a very limited range of the lateral distribution, it will be very difficult if not impossible to reveal the appropriate function in detail. This task can more easily be accessed at LOFAR and AERA which can measure the radio amplitude with many antennas up to lateral distances of a few $\mathrm{km}$.

\subsection{Event selection}

Several selections of LOPES events have been used to study the lateral distribution of air shower induced radio pulses. On the one hand the selection of [47] has been re-used for the comparison of LOPES data to REAS simulations, in order to show the progress made from REAS2 to REAS3. It consists of about 100 events recorded with the east-west oriented LOPES 30 setup in 2005 and 2006 (cf. section 3.2.1). On the other hand, a combination of several new selections has been used to maximize the statistical significance of the results. These selections cover all LOPES data from 2005 to 2009, including events triggered by the KASCADE array as well as events triggered by the Grande extension. However, not the trigger source, but the reconstructed shower core position decides whether an event is considered as KASCADE or Grande event. Since the additional Grande trigger for LOPES was installed during the LOPES 30 operation, the fraction of Grande events is $\sim 55 \%$ higher for the LOPES Dual setup. 
Table 7.2.: Preselection cuts on LOPES events with core in the

\begin{tabular}{lcc} 
& KASCADE array & Grande extension \\
\hline successful reconstruction $^{a}$ & $0.4<$ age $<1.4$ & $-0.385<$ age $<1.485$ \\
core position & $\sqrt{x^{2}+y^{2}}<90 \mathrm{~m}$ & $-420 \mathrm{~m}<x<-50 \mathrm{~m}$, \\
& $-550 \mathrm{~m}<y<-30 \mathrm{~m}$ \\
& $<45^{\circ}$ \\
zenith angle & $>10^{17} \mathrm{eV}$ \\
primary energy $^{b}$ & $\left|E_{\text {atm }}\right|<3000 \mathrm{~V} / \mathrm{m}$ \\
atmospheric electric field $^{c}$ &
\end{tabular}

${ }^{a}$ Further hardware related cuts are included, as listed in [127].

${ }^{b}$ This implicitly includes a cut on the muon and electron number.

${ }^{c}$ For events before 24 August 2006, there is no cut applied on the atmospheric electric field at ground, since no reliable measurement is available.

Table 7.3.: Selection statistics: number of KASCADE and Grande events recorded with different LOPES setups, after certain quality cuts

\begin{tabular}{lccc} 
& KASCADE & Grande & Overlap $^{a}$ \\
\hline LOPES 30 EW & & & \\
total $($ preselection) & 319 & 708 & 4 \\
$\left|E_{\text {atm }}\right|<3000 \mathrm{~V} / \mathrm{m}$ & 318 & 707 & 4 \\
radio reconstruction successful & 315 & 680 & 4 \\
CC-amplitude/RMS $(\mathrm{CC})>14 \cdot \sqrt{\# \text { ant } / 30}$ & 107 & 61 & 1 \\
fraction of correlated power $>80 \%$ & 93 & 47 & 1 \\
Quality cuts on lateral distribution: & & & \\
$R_{0}>1 \mathrm{~m}$ & 78 & 42 & 0 \\
$\epsilon_{100}>1 \mu \mathrm{V} / \mathrm{m} / \mathrm{MHz}$ & 78 & 41 & 0 \\
$R_{0}<3000 \mathrm{~m}$ & 70 & 39 & 0 \\
$\epsilon_{100}<1000 \mu \mathrm{V} / \mathrm{m} / \mathrm{MHz}$ & 70 & 31 & 0 \\
$R_{0}<1350 \mathrm{~m}{ }^{b}$ & 69 & 30 & 0 \\
\hline LOPES Dual EW & & & \\
total $($ preselection) & 664 & 2277 & 15 \\
$\left|E_{\text {atm }}\right|<3000 \mathrm{~V} / \mathrm{m}$ & 653 & 2242 & 15 \\
radio reconstruction successful & 616 & 2103 & 14 \\
CC-amplitude/RMS $(\mathrm{CC})>14 \cdot \sqrt{\# \text { ant } / 30}$ & 261 & 195 & 10 \\
fraction of correlated power $>80 \%$ & 220 & 168 & 8 \\
Quality cuts on lateral distribution: & & & \\
$R_{0}>1 \mathrm{~m}$ & 201 & 149 & 8 \\
$\epsilon_{100}>1 \mu \mathrm{V} / \mathrm{m} / \mathrm{MHz}$ & 200 & 149 & 8 \\
$R_{0}<3000 \mathrm{~m}$ & 175 & 142 & 4 \\
$\epsilon_{100}<1000 \mu \mathrm{V} / \mathrm{m} / \mathrm{MHz}$ & 174 & 134 & 4 \\
$R_{0}<1350 \mathrm{~m}{ }^{b}$ & 169 & 134 & 4 \\
\hline
\end{tabular}

\footnotetext{
${ }^{a}$ In case of overlap, the LOPES analysis is based on the Grande reconstruction.

${ }^{b}$ Cut only applied for the analysis of systematic effects in section 7.3. In the comparison to simulations, for $R_{0}>1350 \mathrm{~m}, R_{0}$ is set to $1350 \mathrm{~m}$ and its error $\Delta R_{0}$ to $300 \mathrm{~m}$.
} 


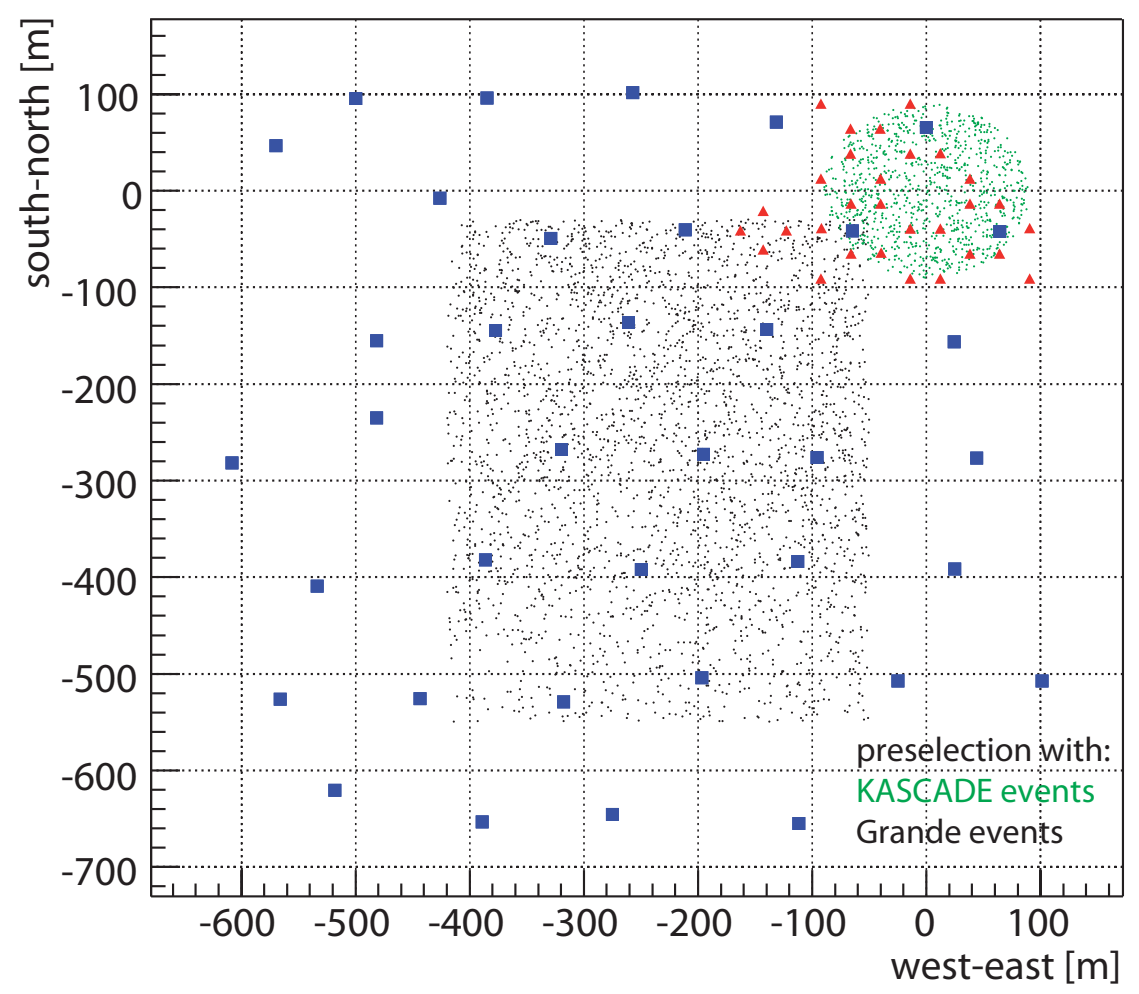

Figure 7.5.: Map of all preselected events. For orientation, also the positions of the LOPES antennas until 2006 (red triangles), and the locations of the Grande detector stations (blue squares) are shown.

Several cuts have been applied on quantities of the KASCADE-Grande reconstruction, based on KRETA version 1.18/05. These cuts include quality checks for a successful reconstruction and limitations to the zenith angle and core position to avoid undefined large uncertainties of the reconstructed parameters [127]. Furthermore, only events with energies above $10^{17} \mathrm{eV}$ have been considered, using the energy determination methods of [128] and [129] (see table 7.2, and figure 7.5). The KASCADE and Grande selections are not totally disjunct, and have a certain overlap with the old LOPES 30 selection of [47].

Moreover, a cut on the atmospheric electric field at ground is applied, because LOPES measurements are not only affected by nearby thunderstorms, but also by atmospheric electric fields $\left|E_{\text {atm }}\right|>3000 \mathrm{~V} / \mathrm{m}$ [31]. In addition, some data have been excluded due to known experimental issues, e.g., malfunction of antennas or maintenance.

All preselected events have been processed with the LOPES standard analysis pipeline (see section 3.2.2). In a few cases the reconstruction failed due to the high noise level at LOPES. Additional cuts have been applied on the cross-correlation beam with the aim to select only those events which can clearly be distinguished from noise. A cut is applied on the fraction of correlated power to exclude events with a high cross-correlation amplitude due to random coherence (cf. section 4.2): The cross-correlation amplitude at the time of the maximum has to be at least $80 \%$ 

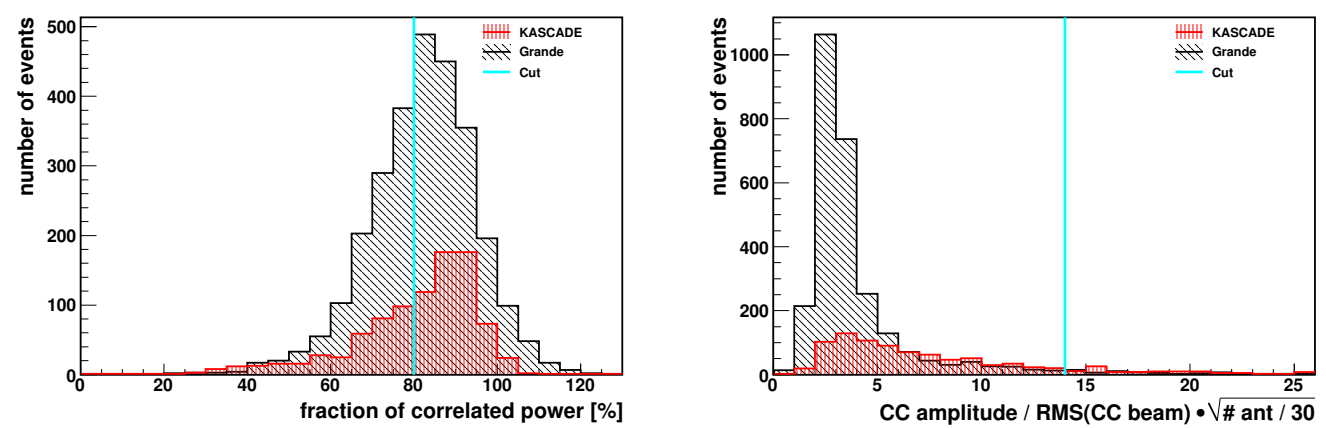

Figure 7.6.: Quality cut on cross-correlation beam; left: fraction of correlated power (= amplitude of the cross-correlation beam / amplitude of the power beam); right: normalized signal-to-noise ratio of the cross-correlation beam (= amplitude of the cross-correlation beam / RMS of the crosscorrelation beam $\cdot \sqrt{\# \text { ant } / 30})$.

of the power beam amplitude. Moreover, a minimum ratio of the cross-correlation beam to its root mean square is required (see figure 7.6). This cut is normalized with a factor of $\sqrt{\# a n t}$, since the signal-to-noise ratio of the cross-correlation beam is proportional to the number of the antennas, \#ant, but the noise dependent error of a non-interferometric analysis usually is only proportional to $\sqrt{\# a n t}$. With this normalization, the fraction of events surviving all cuts is about the same for the LOPES 30 setup (30 east-west aligned antennas) and the LOPES Dual setup (15 east-west aligned antennas). In any case, the normalization factor only marginally affects the selection and the results obtained with the lateral distributions.

Finally, additional quality cuts are applied on the fit parameters of the lateral distribution itself. To make the results of this thesis comparable with earlier analyses of LOPES lateral distributions, the cuts used in [47] have been applied. They reject events for which the fit of the exponential function yields unreasonably low or large values of the fit parameters $\epsilon$ and $R$. Since only the east-west polarization component of the radio emission is analyzed here, all cuts are applied only to quantities reconstructed with the east-west aligned antennas. The north-south component has recently been analyzed and compared to the east-west component in [85].

\subsection{Systematic effects}

Testing theoretical models for the radio emission of air showers requires a measurement precision which is at least of the same order by which certain emission mechanisms contribute to the total radio emission. For instance, the radio emission due to the variation of the charge excess in the shower is predicted to be $\sim 20$ times weaker than the geomagnetic emission [124, 123]. Furthermore, the differences in the lateral distributions of proton and iron showers are also $\sim 10 \%$. Thus, it is worthwhile to understand and control any systematic uncertainties up to a level of at least $20 \%$. This is in principle achievable for LOPES, as the limit due to the uncertainties of 
the amplitude calibration is $5 \%$ (cf., section 4.1).

Thus, several systematic influences on the amplitude and slope parameter of lateral distributions, $\epsilon_{100}$ and $R_{0}$, are investigated in this section. However, even if all systematics are under control, LOPES will still be limited by the high ambient noise and its small lateral extension. Many questions are going to be answered by the next generation of digital radio arrays, like LOFAR or AERA, which both will have a larger lateral extension. In addition, AERA has a better signal-to-noise ratio than LOPES, and LOFAR a denser antenna spacing, which allows detailed investigations of the lateral distributions of individual events. Consequently, when studying systematic effects, not only LOPES is in the focus, but also LOFAR and AERA. Furthermore, understanding and controlling systematic effects is crucial for the reproducibility and comparability of all results.

\subsubsection{Antenna height and shower inclination}

The antenna height ${ }^{1}$ in shower coordinates $z_{s}$ depends on the antenna height above ground $z$ and the shower inclination, i.e., the zenith angle $\theta$. Thus, it makes sense to study the systematic impact of $z$ and $\theta$ on the lateral distribution together. So far, the antenna height ( $z$ as well as $z_{s}$ ) had been completely ignored in the analysis of the lateral distribution. For the antenna height above ground, $z$, this is, without doubt, justified as all LOPES antennas stand within $\sim 1 \mathrm{~m}$ in the same plane. For inclined showers the antenna height in shower coordinates is $z_{s} \gg z$, and it is not obvious that also $z_{s}$ can be neglected in the analysis. The influence of $z_{s}$ on the amplitude of the radio pulse will be maximum at the edges of the array. It can be estimated in the following way.

The effect of $z_{s}$ becomes larger with the inclination of the air shower. For showers with a zenith angle of $40^{\circ}$, the height difference $\Delta z_{s}$ in shower coordinates between two LOPES antennas at opposite edges of the array can be up to $\Delta z_{s} \approx 250 \mathrm{~m}$. $\sin \left(40^{\circ}\right) \approx 160 \mathrm{~m}$, as the maximum extension of the LOPES array is about $250 \mathrm{~m}$. For a simple approximation, it is assumed - like in the LOPES standard analysis pipeline - that the radio emission originates from a single point on the shower axis at a certain height, and that the field strength of a spherical radio emission decreases linearly with distance. Then, the ratio between $\Delta z_{s}$ and the height of the origin of the radio emission (i.e., the curvature radius $r_{c}$ ) defines the maximum effect of $\Delta z_{s}$ on the radio amplitude. For a low curvature radius of $2 \mathrm{~km}$, this can be up to $\Delta z_{s} / r_{c}=160 \mathrm{~m} / 2 \mathrm{~km}=8 \%$. For a typical shower with a zenith angle of $30^{\circ}$ and $r_{c}=5 \mathrm{~km}$, the maximum effect is $\Delta z_{s} / r_{c}=250 \mathrm{~m} \cdot \sin \left(30^{\circ}\right) / 5 \mathrm{~km}=2.5 \%$.

If the core is inside the antenna array, this effect will result in an increased scattering of the measured amplitude at a fixed lateral distance. Thus, the effect will partially average out when fitting a lateral distribution function. However, the slope of lateral distributions will change if the core is not in the center of the array. Consequently, especially lateral distributions of Grande events should be corrected for this effect. But this is difficult, as the systematic uncertainty of $r_{c}$ is very large, resulting in an uncertainty of the correction factor $\Delta z_{s} / r_{c}$ of the same order as the correction factor itself. Thus, no correction is applied in this thesis. However, when comparing

\footnotetext{
${ }^{1}$ The term 'antenna height' is strictly meant in the sense of altitude, and must not be confused with the concept of an 'effective antenna height' related to the antenna gain.
} 

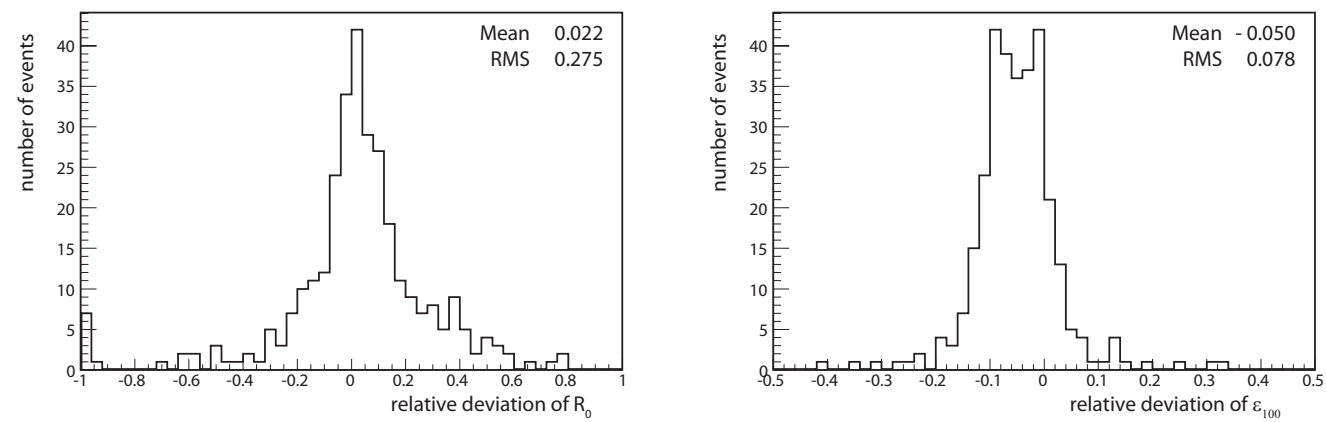

Figure 7.7.: Influence of the pulse distortion by the LOPES bandpass filter on the slope parameter $R_{0}$ and the amplitude parameter $\epsilon_{100}$ of lateral distributions. Plotted is the relative deviation between correcting and not correcting for the pulse distortion in the analysis pipeline: (uncorrected - corrected)/uncorrected. Hence, negative values mean that the results have decreased due to the pulse distortion of the filters.

data to simulations this plays a minor role, as ignoring the antenna height affects simulation and measurement approximately in the same way.

Concluding, the error made in the lateral distribution by ignoring the antenna height and shower inclination is only a few percent for small arrays with an extension of $\sim 200 \mathrm{~m}$. For LOPES, it is typically of the same order as the amplitude calibration uncertainty, or even smaller. Nevertheless, for larger arrays, like LOFAR or AERA, or arrays at a higher altitude, and hence, closer to the radio emission, the effect can become important.

\subsubsection{Pulse distortion}

To check the impact of the pulse distortion by the dispersion of the bandpass filter (cf., section 4.5), all events have been processed a second time, but without applying the phase corrections which are considered in the standard analysis pipeline to account for the pulse distortion by the filter (see figure 7.7).

The pulse distortion can have an effect on the slope and amplitude parameter of lateral distributions. However, the average impact on the slope parameter $R_{0}$ is small and will play a minor role in any statistical analysis of a large data set. It is only of importance for analyzing individual events. On the other hand, the pulse distortion by the bandpass filter results in a systematically lower amplitude parameter $\epsilon_{100}$. This effect is in the same order as the calibration uncertainty due to environmental effects $(\sim 5 \%)$. Consequently, this effect has to be taken into account, especially, when the absolute amplitude is compared to simulations.

Up to now, no correction for the dispersion of the antenna and signal cables is implemented in the standard analysis pipeline. This is justified since the total pulse dispersion of LOPES is dominated by the bandpass filter. Thus, the remaining effects of the antenna and cable are smaller than the amplitude calibration uncertainty, and therefore neglected. 


\subsubsection{Frequency band}

To compare results from experiments with different observing frequency band, it is important to know, how the choice of a certain frequency band affects the average amplitude and the slope of lateral distributions. The average amplitude should be higher for measurements at lower frequencies due to the decreasing spectrum of the air shower radio emission (cf., figure 2.4). Moreover, also the slope of the lateral distribution should depend on the frequency band, since the pulse spectrum is expected to change with lateral distance.

These effects have been studied analogously to other systematic influences, like noise and pulse distortion. All events have been filtered in software with a Hanning window to an effective band of $43-59 \mathrm{MHz}$ and $58-74 \mathrm{MHz}$, respectively. By comparing the amplitude and slope parameters $\epsilon_{100}$ and $R_{0}$ reconstructed with the lower and upper frequency band to the parameters reconstructed with the full band of $43-74 \mathrm{MHz}$, frequency dependent systematic effects are revealed.

However, the results of this analysis are not very conclusive. For each subband, only about half of the events are reconstructible and pass the quality cuts. Different events pass the quality cuts for both subbands which induces a systematic uncertainty in the comparison, and hampers quantitatively reliable statements. There are two explanations for this: First, each subband contains only a part of the total pulse power. Second, the pulse broadens by the filter response which decreases the time resolution. This in turn results in a lower quality of the cross-correlation beam. Hence, more events fail the quality cuts on the cross-correlation amplitude. Nevertheless, mean effects on $\epsilon_{100}$ and $R_{0}$ have been evaluated.

The mean $\epsilon_{100}$ reconstructed with the lower subband is $58.5 \%$ and the mean $\epsilon_{100}$ of the higher subband $47.6 \%$ of the mean $\epsilon_{100}$ reconstructed with the full bandwidth. This is consistent with a decreasing frequency spectrum as expected from simulations, and as observed by a previous LOPES spectral analysis [130]. On the other hand, the mean $R_{0}$ remains unchanged within $1 \%$ for both subbands. However, due to the discussed difficulties, the significance of the results is questionable. At LOPES, the noise level seems to be too high for this kind of subband analysis. At AERA, the total bandwidth available is larger $(30-80 \mathrm{MHz})$, and the noise level lower. Thus, it will be worthwhile to revisit this topic with AERA data.

\subsubsection{Up-sampling}

Up-sampling is one of the major data conditioning steps in the LOPES standard analysis pipeline (cf., section 3.2.2). Up-sampling is the correct way to interpolate a band-limited signal and is necessary to improve the timing resolution of LOPES (see chapter 4, appendix B). Otherwise, the precision of pulse arrival time measurements would be limited by the sampling frequency of LOPES $(80 \mathrm{MHz})$, and not by the relative timing uncertainty of $2 \mathrm{~ns}$. The relevance of up-sampling for analyzing lateral distributions of pulse amplitudes is investigated by the following studies:

All events have been processed with different sampling frequencies from $80 \mathrm{MHz}$ (no up-sampling) to $2560 \mathrm{MHz}$ (up-sampling by a factor of 32). For each tested sampling frequency, the slope and amplitude parameters $\epsilon_{100}$ and $R_{0}$ have been compared to the ones obtained with the default up-sampling factor of $16(1280 \mathrm{MHz})$. The effect 


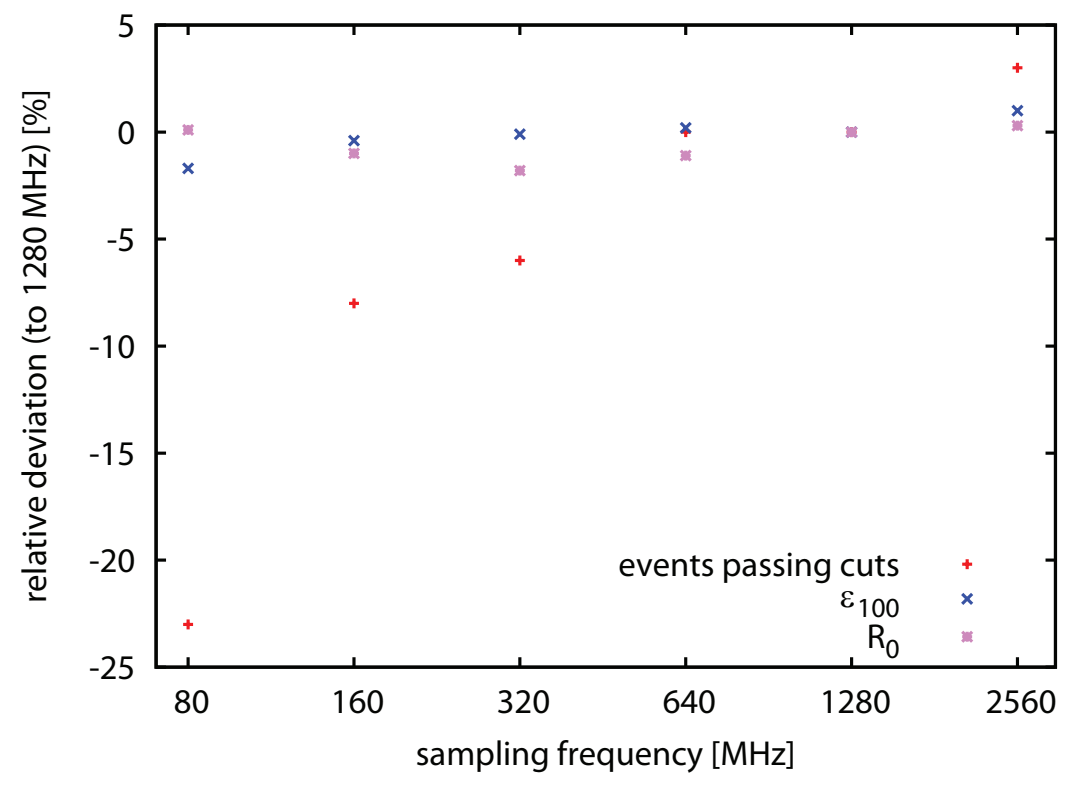

Figure 7.8.: Influence of up-sampling on lateral distributions: mean relative deviation to the results obtained with the default up-sampling frequency of $1280 \mathrm{MHz}$. The sampling frequency only marginally effects the amplitude and slope parameters $\epsilon_{100}$ and $R_{0}$, but has a significant effect on the number of events passing the quality cuts on the cross-correlation beam. No errors are given because the standard deviation is completely determined by a few outliers.

Table 7.4.: Summary of systematic effects on the mean amplitude and slope parameters $\epsilon_{100}$ and $R_{0}$ of lateral distributions at LOPES.

\begin{tabular}{|l|l|}
\hline Noise (chapter 6) & $\begin{array}{l}\text { flattens lateral distributions by } \\
\text { by } \lesssim 5 \% \text {; unimportant for events with high SNR }\end{array}$ \\
\hline $\begin{array}{l}\text { shower inclination, } \\
\text { antenna height }\end{array}$ & negligible for LOPES, but important for larger arrays \\
\hline Pulse distortion & on average marginal effect on $R_{0}$; decreases $\epsilon_{100}$ by $\sim 5 \%$ \\
\hline Frequency band & $\begin{array}{l}\text { influence unclear for LOPES; seems to effect only } \epsilon_{100}, \\
\text { which is higher at lower frequencies }\end{array}$ \\
\hline Up-sampling & $\begin{array}{l}\text { important for preceding cross-correlation beamforming, but } \\
\text { on average no effect on lateral distributions }\end{array}$ \\
\hline Reconstruction & possible bias and unknown systematic uncertainties \\
\hline
\end{tabular}

is negligible as $\epsilon_{100}$ and $R_{0}$ stay constant within $2 \%$ (see figure 7.8 ). Hence, upsampling is of minor importance for the reconstruction of lateral distributions - at least, if the signal is measured as maximum of a Hilbert envelope. Other methods might be more sensitive to the sampling frequency.

However, up-sampling is of great importance for cross-correlation beamforming, which precedes any lateral distribution analysis in the case of LOPES. If the up- 
sampling frequency is too low ( $\lesssim 640 \mathrm{MHz}$ ), a reasonable fraction of events fails the quality cuts on the cross-correlation beam. This is not surprising, because crosscorrelation beamforming requires a relative timing resolution of $\sim 1 \mathrm{~ns}$ (cf., chapter 4). Thus, for LOPES, up-sampling is mandatory in any case. For other experiments, like AERA, up-sampling will not be essential to reconstruct lateral distributions, since the analysis is not necessarily based on interferometry.

\subsection{Comparison of LOPES data and REAS3 simulations}

Per-event comparisons between measured lateral distributions and simulations are an excellent way to test theoretical models. A comparison of LOPES lateral distributions with REAS3 Monte Carlo simulations shows that they are qualitatively and quantitatively in relatively good agreement. Moreover, REAS3 predicts a steeper slope for lateral distributions of proton showers than for iron showers. Hence, comparing measured with simulated slopes yields a model-dependent way to estimate the primary mass composition. ${ }^{2}$

\subsubsection{Test of REAS3 simulations against LOPES data}

In reference [47], LOPES lateral distributions have been compared to REAS2 simulations for proton primaries, where a selection of 130 LOPES 30 events of 2005 and 2006 was used. 84 of them pass all quality cuts, and are reused in the present studies. The lateral distribution is reconstructed for these events, including all the improvements to the LOPES standard analysis pipeline made since [47], e.g., the better noise treatment (cf., chapter 6). Moreover, the same LOPES events are now compared to REAS3 simulations for proton and iron primaries (see figure 7.9 for examples). All results presented here have been cross-checked with the REAS3 simulations used in chapter 8, and no major difference is observed. Thus, only the REAS3 simulations for the events presented in reference [47] are shown, since they can be compared directly to the previous REAS2 simulations.

For the REAS3 simulations, the same procedure has been applied as for the REAS2 simulations of reference [47]: Each REAS simulation needs a CORSIKA air shower simulation as input. For this simulation, the arrival direction and energy reconstructed by KASCADE-Grande for each individual event are used as input parameters, and proton or iron nuclei as primary particles. First, 250 showers have been simulated for each event with CONEX [131], with a fixed primary energy, direction and mass. Of these 250 showers, only one with a typical shower maximum $X_{\max }$ (i.e., $X_{\max } \approx<X_{\max }>$ ) is chosen as input for a full CORSIKA and REAS simulation, since a full simulation is time-consuming (a few days per event on a normal personal computer of the year 2010). For all simulations, QGSjet II [132] was used as high energy interaction model and UrQMD [133] as low energy interaction model.

The amplitude at each simulated antenna position is determined as maximum of the east-west polarization component of the electric field strength in the effective bandwidth of LOPES $(43-74 \mathrm{MHz})$. Since the east-west aligned LOPES antennas

\footnotetext{
${ }^{2}$ A summary of this section is published in Astrophysics and Space Sciences Transactions 7 (2011) 303-306 [53].
} 


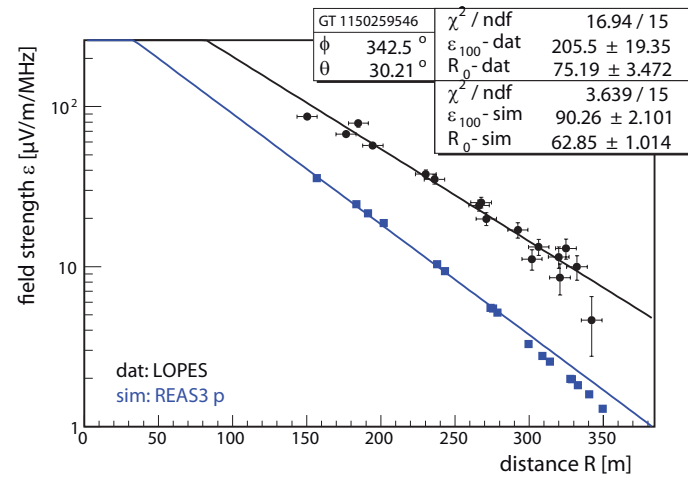

(a) steep event, REAS3 proton

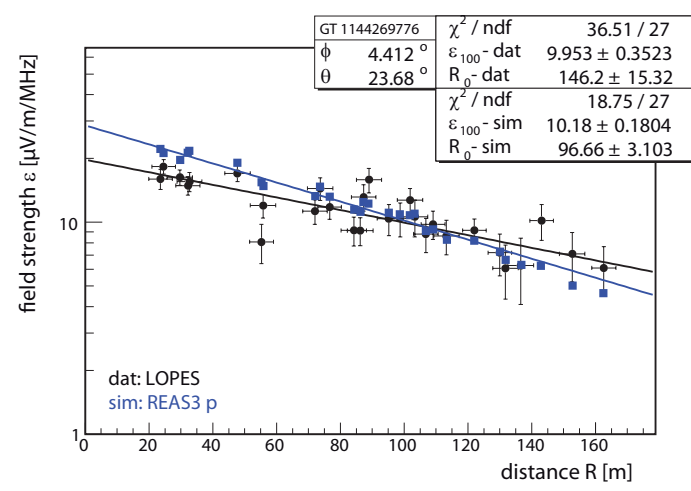

(c) typical event, REAS3 proton

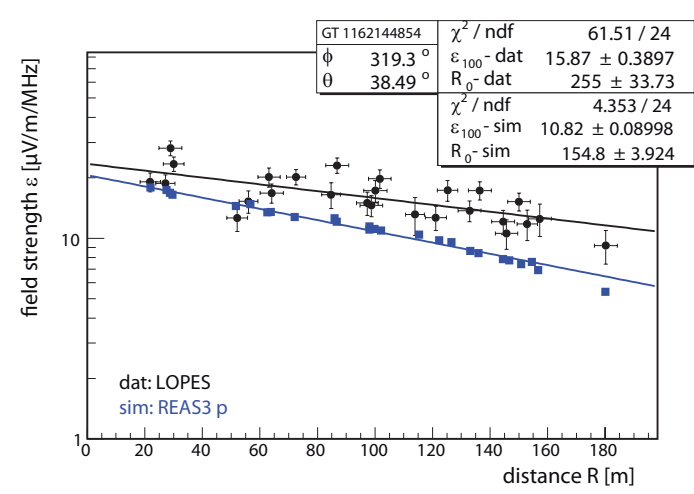

(e) flat event, REAS3 proton

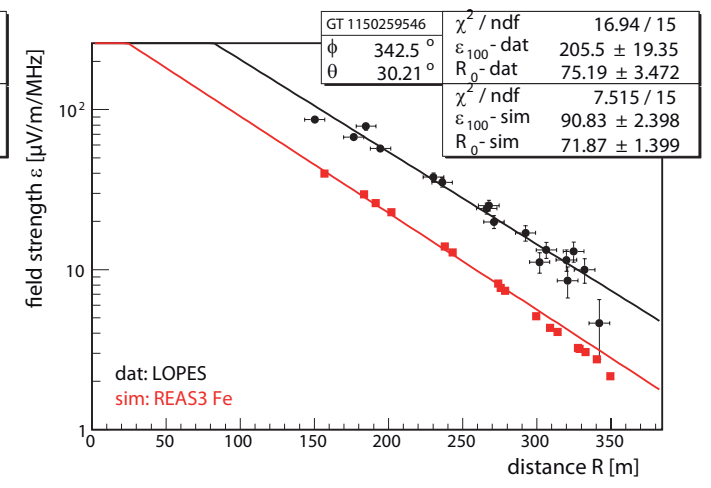

(b) steep event, REAS3 iron

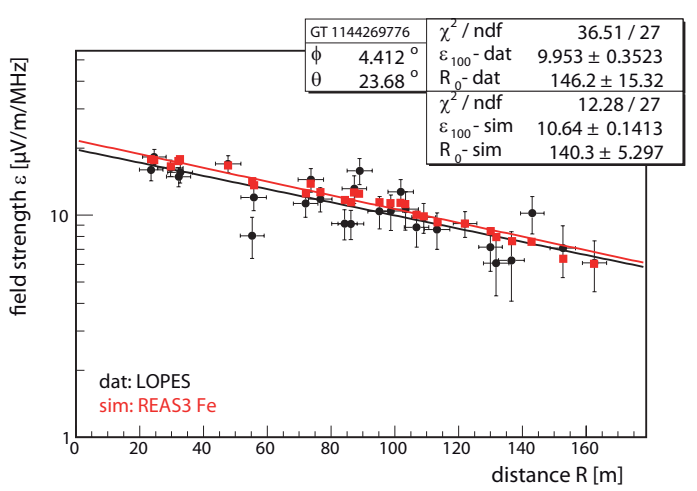

(d) typical event, REAS3 iron

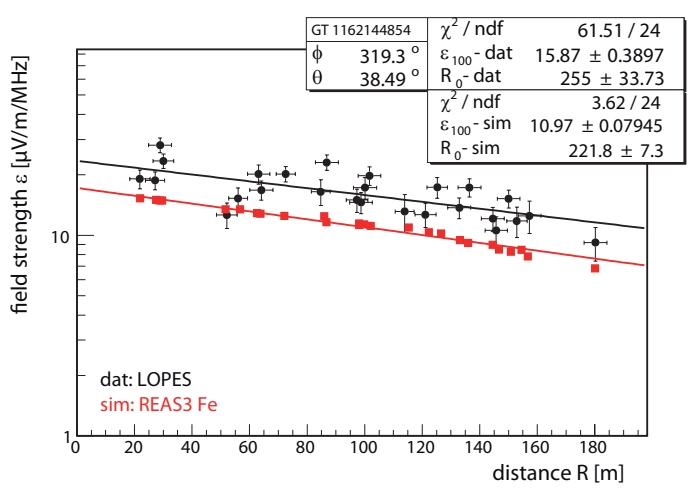

(f) flat event, REAS3 iron

Figure 7.9.: Example events for the comparision of LOPES lateral distributions to REAS3 simulations for proton and iron primaries. For iron simulations, the slope parameter $R_{0}$ is generally closer to the LOPES data than for proton simulations. The amplitude parameter $\epsilon_{100}$ is consistent with LOPES data for proton and iron simulations, since it has a large systematic uncertainty of $35 \%$ due to the amplitude calibration, and $20-40 \%$ due to the energy uncertainty of KASCADE-Grande. 
have also a certain sensitivity to the vertical polarization component, this is only an approximation to the experimental situation. Future studies with the Offline software framework will show how good this approximation is, because in contrast to the LOPES standard analysis pipeline, Offline is able to treat the electric field strength correctly as a vectorial quantity.

The comparison of the reprocessed LOPES events to REAS2 simulations confirms the results of reference [47]: REAS2 predicts the correct order of magnitude for the amplitude parameter $\epsilon_{100}$ (see figure 7.10). However, the large fluctuations of $\epsilon_{100}$ in the REAS2 simulations are incompatible with LOPES data. These fluctuations are caused by a large asymmetry of the radio amplitude with respect to the azimuthal observer position relative to the shower axis. REAS3 predicts a much smaller asymmetry [36], and thus also smaller fluctuations of $\epsilon_{100}$. Furthermore, REAS2 lateral distributions are too steep. This has changed with REAS3 which is in better agreement with LOPES data.

The amplitude parameter $\epsilon_{100}$ of REAS3 simulations agrees with $\epsilon_{100}$ of the measured lateral distributions within the systematic uncertainty of the amplitude calibration. The absolute scale of the measured amplitude is only known within a relatively large uncertainty of $35 \%$ (cf., section 4.1). Thus, a better calibration source would be needed to judge whether there is a significant systematic offset between measured and simulated amplitudes. Also other explanations for the offset in the amplitude are conceivable: For example, an underestimation of the electron number in the shower or an unconsidered emission mechanism could also explain, why the simulated amplitudes are systematically lower than the measured amplitudes. Furthermore, in the present analysis only the most important detector properties are considered in the simulations. It is under investigation [134], how $\epsilon_{100}$ and $R_{0}$ will be affected by considering all detector effects in the simulations, including the antenna gain pattern.

For a few single events, the simulated amplitude deviates by much more than the absolute calibration uncertainty of $35 \%$. This may partially due to reasons discussed above, but needs additional explanation. For all REAS simulations a CORSIKA shower with a typical $X_{\max }$ has been chosen, but a few of the reals showers ought to be in the tails of the $X_{\max }$ distribution. Thus, the present REAS3 simulations are expected to reproduce only the mean of $\epsilon_{100}$ and $R_{0}$, but not each individual event. Indeed, if a CORSIKA shower is selected as input which reproduces the muon number measured by KASCADE-Grande, there are less outliers. Another explanation for the outliers might be the larger uncertainty of the primary energy in the KASCADEGrande reconstruction $(20-40 \%)$ for individual events. Moreover, it cannot be excluded that the selection contains single thunderstorm events, since a part of the events was recorded before the thunderstorm monitoring was installed at LOPES. In particular, for the event with a measured amplitude more than 10 times higher than the simulated amplitude, a thunderstorm cannot be excluded. Consequently, REAS3 simulations are in general compatible with LOPES data with respect to $\epsilon_{100}$, independent of which primary nucleus is assumed.

Also with respect to the slope parameter $R_{0}$, REAS3 simulations are much closer to LOPES data than REAS2. For REAS3, a clear correlation between the simulated and measured slope is visible. This shows that the principle dependencies of radio lateral distributions on shower parameters can be described by REAS3. However, REAS3 does not predict flat lateral distributions to the same extent as they are 


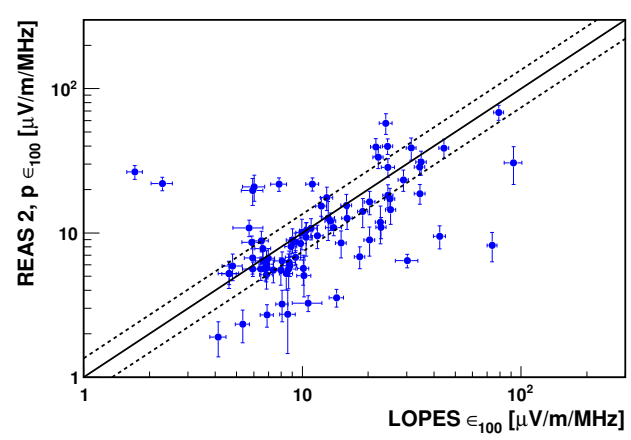

(a) amplitude, REAS2 proton

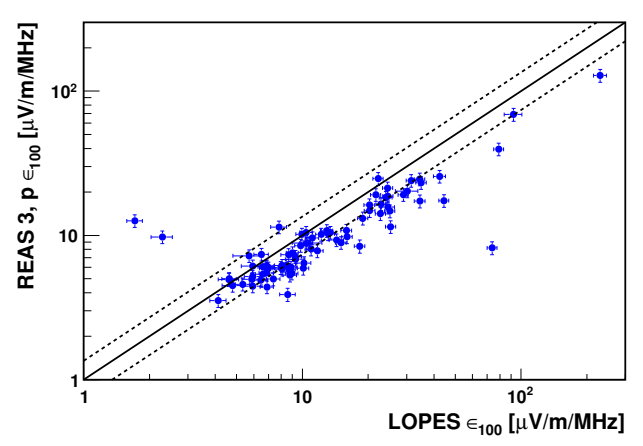

(c) amplitude, REAS3 proton

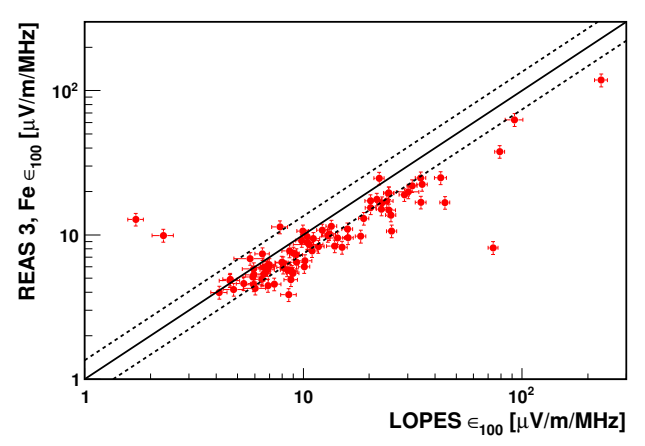

(e) amplitude, REAS3 iron

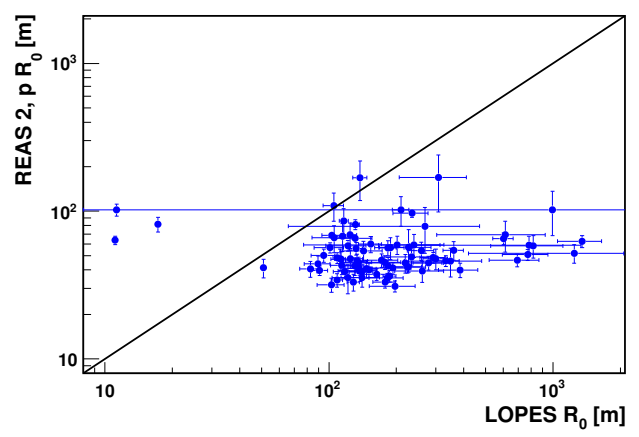

(b) slope, REAS2 proton

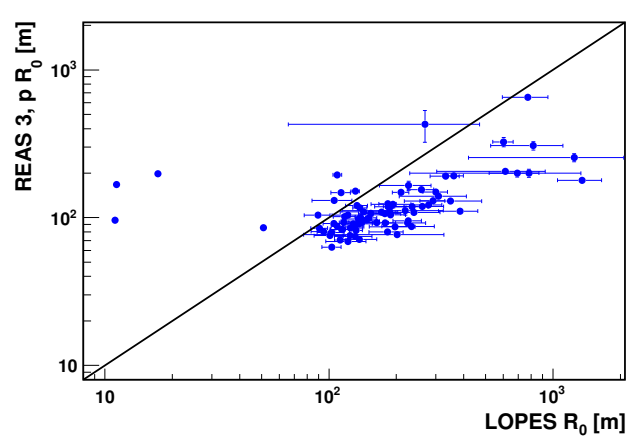

(d) slope, REAS3 proton

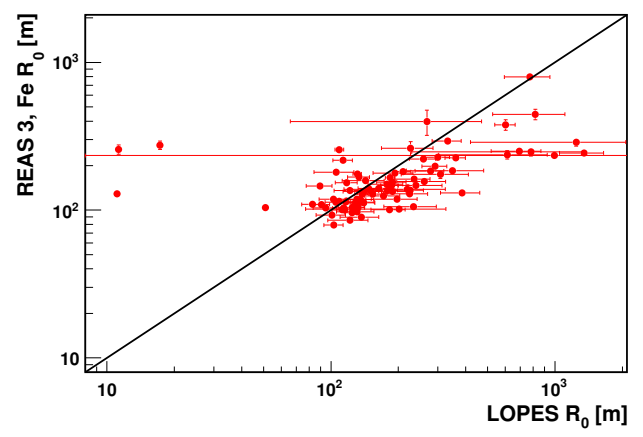

(f) slope, REAS3 iron

Figure 7.10.: Comparison of the amplitude and slope parameters between LOPES measurements and different REAS simulations. Error bars are the statistical error of the fit to the individual lateral distributions. The dashed lines indicate the absolute amplitude calibration uncertainty. The number of events in the plots ranges from 81 to 83 , since for 1 to 3 events the fit to the simulated lateral distributions fails. Single outliers could be due to the energy uncertainty of the KASCADE-Grande reconstruction used as input for the REAS simulations, or due to thunderstorms. 

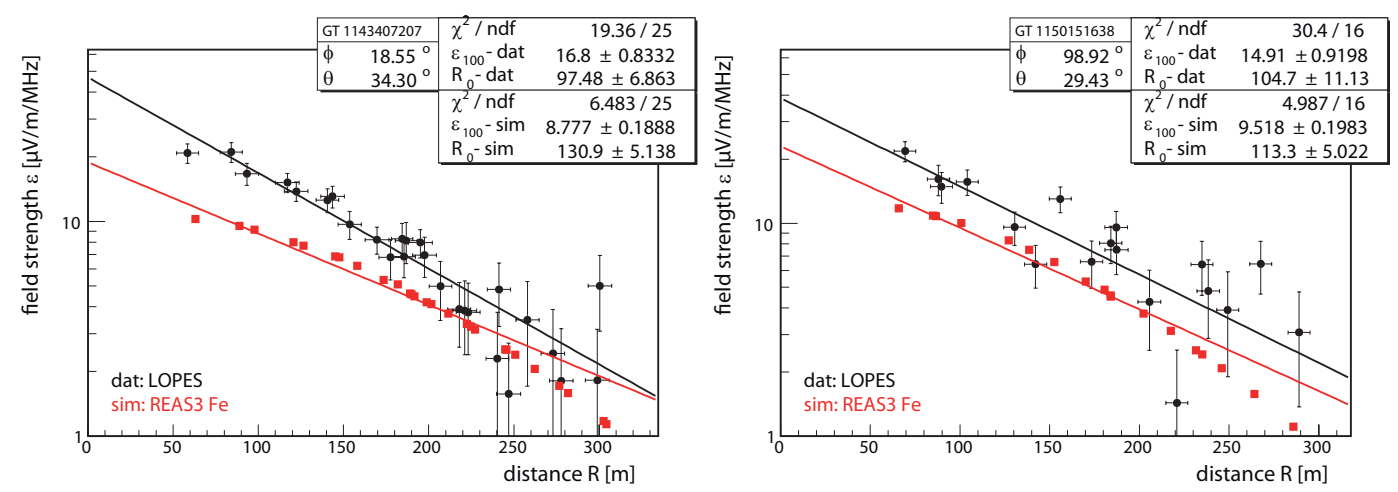

Figure 7.11.: Two examples for which REAS3 indicates a flattening of the lateral distribution towards the shower core: LOPES data and the corresponding REAS3 simulations for iron primaries.

measured with LOPES. It is difficult to decide, however, if this disagreement is due to the physics modeled with REAS3, or due to systematic effects in the LOPES measurement. There is a principle difficulty to measure slope parameters which are much larger than the lateral extension of the experiment ( $\sim 200 \mathrm{~m}$ for LOPES).

Furthermore, a bias due to the selection cuts cannot be excluded, since a possible dependence of the cross-correlation beam on the lateral distribution has not been investigated, yet. However, it is not clear whether a possible bias would favor steep or flat lateral distributions. Either the reconstruction could be more likely to fail, if only few antennas have a high signal-to-noise ratio, which is the case for steep lateral distributions. On the other hand, it is conceivable that a few antennas with a good signal-to-noise ratio could make the cross-correlation beam more reliable than many antennas with an average signal-to-noise ratio. Thus, it is not clear how trustworthy and significant the disagreement between REAS3 and LOPES lateral distributions for flat events is.

Consequently, the lateral distribution of almost all LOPES events is compatible with REAS3 within the systematic uncertainties. Already in [47] it was observed that a few LOPES events $(\sim 10 \%)$ show a flatting towards the shower core. Also a few REAS3 simulations show a flattening towards the core, but not always for the same events and to the same extent as LOPES (see figure 7.11). Thus, this feature of lateral distributions needs further investigations, which will be performed at AERA and LOFAR. For this kind of studies, in particular LOFAR offers promising opportunities, since it has not only a larger lateral extension than LOPES, but also a finer antenna spacing.

\subsubsection{Mass sensitivity}

Showers initiated by proton primaries are expected to have on average steeper radio lateral distributions than showers initiated by iron primaries, since proton showers on average have a deeper shower maximum ( $\widehat{=}$ larger $\left.X_{\max }\right)$. The mass sensitivity of the lateral slope has also been predicted by REAS2 [135]. This can be utilized to estimate the primary composition by comparing the slope parameter $R_{0}$ of LOPES 

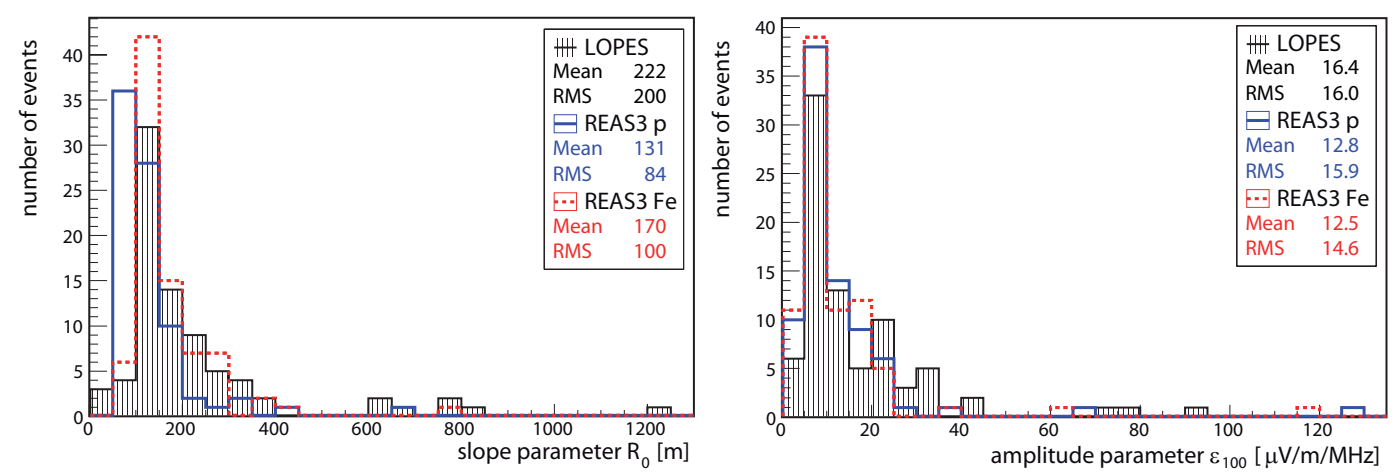

Figure 7.12.: Slope parameter $R_{0}$ and amplitude parameter $\epsilon_{100}$ of lateral distributions: measured lateral distributions of the LOPES 30 selection made in reference [47], and REAS3 simulations for proton and iron primaries. While $R_{0}$ is systematically different for proton and iron showers, $\epsilon_{100}$ is about the same.

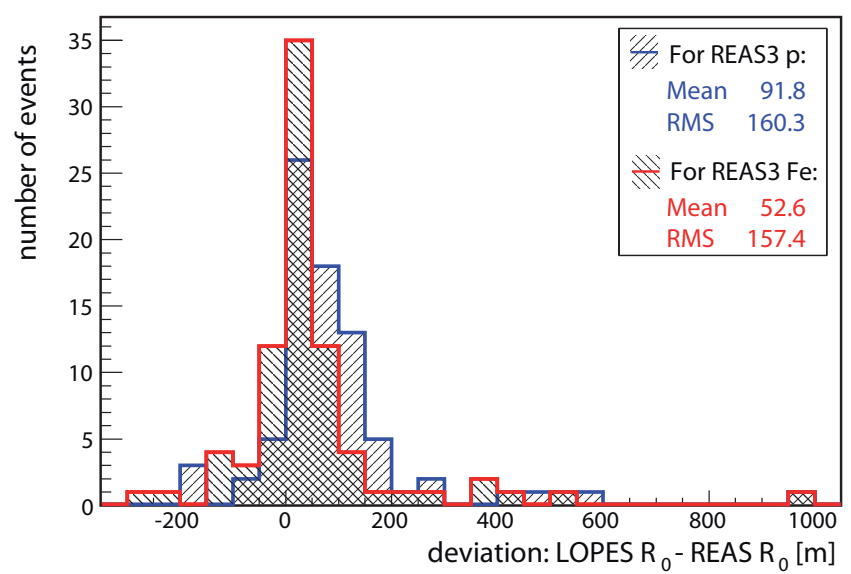

Figure 7.13.: Per-event deviations between the slope parameter $R_{0}$ of REAS3 and LOPES lateral distributions

events with simulations for the same events. Figure 7.12 shows that also for REAS3 the slope parameter $R_{0}$ is systematically larger for the iron simulations. On the other hand, the amplitude parameter $\epsilon_{100}$ is about the same for proton and iron simulations, since it depends mainly on the primary energy and the shower geometry, but only marginally on the primary mass.

The REAS3 lateral distributions for iron primaries match the LOPES data significantly better than the simulations for proton primaries (see figure 7.13). However, it is difficult to estimate the systematic uncertainties in this comparison. In section 7.3 it was shown that many systematic effects influence the slope of lateral distributions by $\lesssim 10 \%$. This is less than the typical difference between proton and iron showers of $\sim 20 \%$. Nevertheless, the unconsidered detector effects in the REAS3 simulations and the model-dependence of the simulations are further sources of uncertainty. A future analysis has to show by how much the hadronic interaction model used for the 
preceding CORSIKA simulations influences the slope of radio lateral distributions. It has already been tested that the effect of selecting a certain CORSIKA shower as input for REAS3 is small - at least as long as distributions of $R_{0}$ and $\epsilon_{100}$ are in the focus, but not individual events. Selecting a CORSIKA shower which can reproduce the muon number reconstructed by KASCADE-Grande instead of a CORSIKA shower with a typical $X_{\max }$ does not significantly change the general results.

\subsection{Conclusion}

The present analysis of LOPES lateral distributions and REAS3 simulations demonstrates the improved understanding of the radio emission. In spite the fact that REAS3 has no free parameters, it can reproduce measured LOPES amplitudes within the absolute calibration uncertainty. Also with respect to the slope parameter of the lateral distribution there is reasonable agreement between LOPES measurements and REAS3 simulations. Thus, REAS3 lateral distributions are generally compatible with LOPES measurements. For the first time, a test of simulations against LOPES data is limited by experimental and systematic uncertainties, and no obvious contradiction was found. Thus, REAS3 lateral distributions are at least a good approximation to the real, physical lateral distributions and can be used to interpret measurements.

The comparison of LOPES lateral distributions with REAS3 simulations based on QGSjet II favors a heavy composition at primary energies of $\sim 10^{17}-10^{17.5} \mathrm{eV}$. However, systematic uncertainties in the measurement and the model dependence of the simulations are still too large to exclude a light, proton-like composition. For this reason, the mass sensitivity of radio lateral distributions is currently being investigated further with LOPES measurements and REAS3 simulations based on different hadronic interaction models [126]. Furthermore, a full consideration of all known detector effects will improve the quality of any comparisons between LOPES data and simulations [134].

Finally, it is necessary to understand the radio emission in even greater detail, and determine how precise the primary composition can be reconstructed with lateral distributions. This requires experiments which are not limited like LOPES by the small baseline and the high ambient noise level. Two leading experiments in the near future will be LOFAR and AERA. AERA provides measurements at larger baselines (up to a few $\mathrm{km}$ ) and benefits from a lower ambient noise level. It can probe the precision of any energy and mass reconstruction method by directly comparing a fraction of the radio events to fluorescence measurements of the same air showers. LOFAR will be crucial to understand the lateral distribution in detail, since it features hundreds of antennas in a very dense array. A combination of the studies at both experiments will reveal whether the lateral distribution of the radio emission yields a method suitable to investigate the composition of the highest energy cosmic rays. Nevertheless, measuring the lateral distribution is not the only way to access the primary composition with digital radio antenna arrays. The next chapter shows an alternative method to determine the shower maximum by measuring the shape of the radio wavefront. 


\section{Pulse Arrival Time Distributions}

The shape of the radio wavefront can be probed with pulse arrival time measurements in individual antennas. So far, the radio wavefront has not been investigated in detail. It does not necessarily have to follow the shape of the particle front (pancake), since the radio emission arises as a coherent sum over the entire shower development. In the LOPES beamforming of the standard analysis pipeline, a spherical wavefront is assumed. Furthermore, also a theoretical study based on REAS2 assumed a spherical wavefront [107]. However, already in reference [89] it was noticed that a spherical wavefront does not perfectly describe the LOPES measurements, and that the angular resolution of LOPES is limited to $\sim 1^{\circ}$ due to the limited knowledge of the exact wavefront shape.

The analysis presented in the following demonstrates that the wavefront is not spherical. Instead, a conical wavefront is a better approximation to LOPES measurements and to REAS3 simulations. While a spherical wavefront corresponds to a simple point source, a conical wavefront corresponds according to Huygens' principle to a point source moving on a straight line, i.e., the shower axis [136]. For an illustrative picture, imagine a ship generating a conical bow wave. Still, also the conical wavefront can only be a simplified approximation of reality, since the air shower has a finite lateral extension and is not a moving point source.

The wavefront shape cannot only be used to test theoretical predictions. In addition, the mass of the primary particle can be estimated by reconstructing the atmospheric depth of the shower maximum $X_{\max }$. Since the cross-section of light nuclei, like protons, is smaller than the cross-section of heavy nuclei, like irons, light nuclei on average interact deeper in the atmosphere than heavy nuclei. Consequently, the average $X_{\max }$ of showers initiated by light nuclei is larger than the average $X_{\max }$ of showers initiated by heavy nuclei. A larger $X_{\max }$ also means that the radio emission is closer to the surface. This results in a large curvature ( $\widehat{=}$ small curvature radius) of the wavefront in the spherical picture, respectively in a small opening angle in the conical picture. A small $X_{\max }$ results in a flat radio wavefront, i.e., a small curvature, respectively a large opening angle close to $180^{\circ}$. This is in principle the same geometrical effect, which also results in a $X_{\max }$ sensitivity of the lateral distribution (cf., chapter 7 and reference [135]).

In reference [107], it has been investigated with REAS2 simulations, how well $X_{\max }$ can be reconstructed by assuming a curved radio wavefront. Under ideal conditions, i.e., a core uncertainty of a few $\mathrm{m}$, an arrival direction uncertainty $\ll 1^{\circ}$, and a low noise level, the error of $X_{\max }$ can be relatively small with $\Delta X_{\max } \lesssim 20 \mathrm{~g} / \mathrm{cm}^{2}$. But under realistic conditions, $\Delta X_{\max }$ can easily exceed $100 \mathrm{~g} / \mathrm{cm}^{2}$. However, it is not clear, whether REAS2 describes the shape of the radio wavefront correctly, especially, since REAS3 simulations clearly favor a conical wavefront.

The wavefront of the radio emission is studied with a selection of LOPES events and REAS3 simulations made under the assumption of proton and iron primaries. For 
Table 8.1.: Selection cuts for pulse arrival time distributions: number of KASCADE and Grande events (cf., table 7.2), recorded with different LOPES setups. The quality cuts are only applied for the reconstruction of $X_{\max }$ (section 8.4), but not for the investigation of the wavefront shape (section 8.3).

\begin{tabular}{|c|c|c|c|}
\hline & KASCADE & Grande & Overlap $^{a}$ \\
\hline \multicolumn{4}{|l|}{ LOPES $30 \mathrm{EW}$} \\
\hline CC beam cuts of table 7.3 & 93 & 47 & 1 \\
\hline KASCADE-Grande mass reconstruction available & 57 & 45 & 0 \\
\hline \multicolumn{4}{|l|}{ Additional cuts for spherical wavefront: } \\
\hline curvature radius $r_{c}<20 \mathrm{~km}$ & 54 & 43 & 0 \\
\hline reduced $\chi^{2}<1.5$ & 45 & 31 & 0 \\
\hline relative error $\Delta \kappa / \kappa<60 \%$ & 36 & 26 & 0 \\
\hline \multicolumn{4}{|l|}{ Additional cuts for conical wavefront: ${ }^{b}$} \\
\hline cone parameter $\rho>0.001 \mathrm{rad}$ & 55 & - & - \\
\hline reduced $\chi^{2}<1.5$ & 43 & - & - \\
\hline relative error $\Delta \rho / \rho<60 \%$ & 32 & - & - \\
\hline \multicolumn{4}{|l|}{ LOPES Dual EW } \\
\hline CC beam cuts of table 7.3 & 220 & 168 & 8 \\
\hline KASCADE-Grande mass reconstruction available & 186 & 165 & 8 \\
\hline \multicolumn{4}{|l|}{ Additional cuts for spherical wavefront: } \\
\hline$\overline{\text { curvature radius } r_{c}<20 \mathrm{~km}}$ & 178 & 163 & 8 \\
\hline reduced $\chi^{2}<1.5$ & 166 & 163 & 8 \\
\hline relative error $\Delta \kappa / \kappa<60 \%$ & 129 & 156 & 8 \\
\hline \multicolumn{4}{|l|}{ Additional cuts for conical wavefront: ${ }^{b}$} \\
\hline cone parameter $\rho>0.001 \mathrm{rad}$ & 181 & - & - \\
\hline reduced $\chi^{2}<1.5$ & 166 & - & - \\
\hline relative error $\Delta \rho / \rho<60 \%$ & 137 & - & - \\
\hline
\end{tabular}

${ }^{a}$ In case of overlap, the LOPES analysis is based on the Grande reconstruction.

${ }^{b}$ Only KASCADE events are used for $X_{\max }$ reconstruction with the cone method, since only for KASCADE events the shower core and thus the apex of the cone is contained in the LOPES array.

the REAS3 simulations, $X_{\max }$ can be reconstructed with a resolution of $\lesssim 30 \mathrm{~g} / \mathrm{cm}^{2}$ by fitting a conical wavefront to the pulse arrival times in individual antennas. For LOPES measurements, the reconstructed $X_{\max }$ is consistent with nuclear cosmic rays. But due to the high noise level and the small extension of LOPES $(\sim 200 \mathrm{~m})$, the uncertainty $\Delta X_{\max }$ is too large for precise per-event mass estimations. Therefore, the aim of the present analysis is not a reconstruction of the composition in the energy range of LOPES $\left(\sim 10^{17}-10^{18} \mathrm{eV}\right)$. Instead, the qualitative investigation of the radio wavefront is in the focus, as well as the development of a method for $X_{\max }$ reconstruction, and the demonstration of its feasibility. Next generation observatories with larger lateral extensions and a better signal-to-noise ratio, like AERA and LOFAR, will have the perspective to exploit the method for quantitative per-event estimations of $X_{\max }$.

The first section of this chapter presents the LOPES events and REAS3 simulations used for analysis. The next section explains how pulse arrival times are measured with LOPES, and how the wavefront shape can be reconstructed from these measurements. 
Section 8.3 discusses the measured and simulated wavefronts, and section 8.4 presents a method of how to reconstruct $X_{\max } \cdot{ }^{1}$

\subsection{Selection: LOPES events and REAS3 simulations}

For the analysis of the pulse arrival time distributions, the same preselection of LOPES events is used as for the lateral distribution of amplitudes (cf. section 7.2). It includes KASCADE and Grande events measured with the east-west aligned antennas of the LOPES 30 and LOPES Dual setups. Furthermore, the same quality cuts on the cross-correlation beam are applied to restrict the analysis to events with a high signal-to-noise ratio.

For each event, two REAS3 simulations have been performed, one for a proton as primary particle and one for an iron nucleus as primary particle. For this, the shower geometry (core and arrival direction) and the primary energy reconstructed by KASCADE-Grande have been used as input. With this input, a number of CONEX air shower simulations have been performed for each event (200 for proton primaries, 100 for iron primaries - due to lower fluctuations) using QGSjet II [132] and UrQMD [133] as interaction models for high and low energies, respectively. The CONEX simulation which can best reproduce the muon number $N_{\mu}$ measured with KASCADE-Grande has been chosen as input for a full CORSIKA and REAS3 simulation. Fixing $N_{\mu}$ still leaves some freedom in the electron number $N_{\mathrm{e}}$ and $X_{\max }$ : Showers with almost identical $N_{\mu}$, cover a relatively large range of $N_{\mathrm{e}}$ and $X_{\max }$. Details of the simulation procedure will be published soon in [138]. In particular, the new procedure is different from the one used in chapter 7 , in which a shower with a typical $X_{\max }$ was chosen. The new procedure has the advantage that a larger range of $X_{\max }$ can be probed in a certain energy range to study the dependency of the wavefront shape on $X_{\max }$.

For the reconstruction of $X_{\max }$ (see section 8.4), additional quality cuts have been applied to the data, as summarized in table 8.1. The aim is to demonstrate that a reconstruction of $X_{\max }$ is in principle possible. Thus a relatively good reconstruction of the curvature $\kappa$, respectively the cone parameter $\rho$ is required. Furthermore, only events are used, for which a reconstruction of the primary mass with KASCADEGrande is available to perform a direct comparison with the reconstructed $X_{\max }$.

\subsection{Analysis procedure}

Reconstructing the shape of the radio wavefront requires knowledge about the geometrical delay $\tau_{\text {geo }}$ of the radio pulse at each antenna. $\tau_{\text {geo }}$ depends not only on the shape of the wavefront, but also on the shower geometry, i.e., the arrival direction and core position (see figure 8.1). The shower core and the arrival direction is known from KASCADE-Grande. For a fixed shower geometry, $\tau_{\text {geo }}$ at each antenna depends only on the shape of the radio wavefront and the antenna position in shower coordinates $\left(R, z_{s}\right)$. $\tau_{\text {geo }}$ can be determined with a measurement of the pulse

\footnotetext{
${ }^{1} \mathrm{~A}$ summary of this chapter is published in Proceedings of the $32^{\text {nd }}$ International Cosmic Ray Conference 2011, Beijing, China, Volume 3, \#0313 [137].
} 


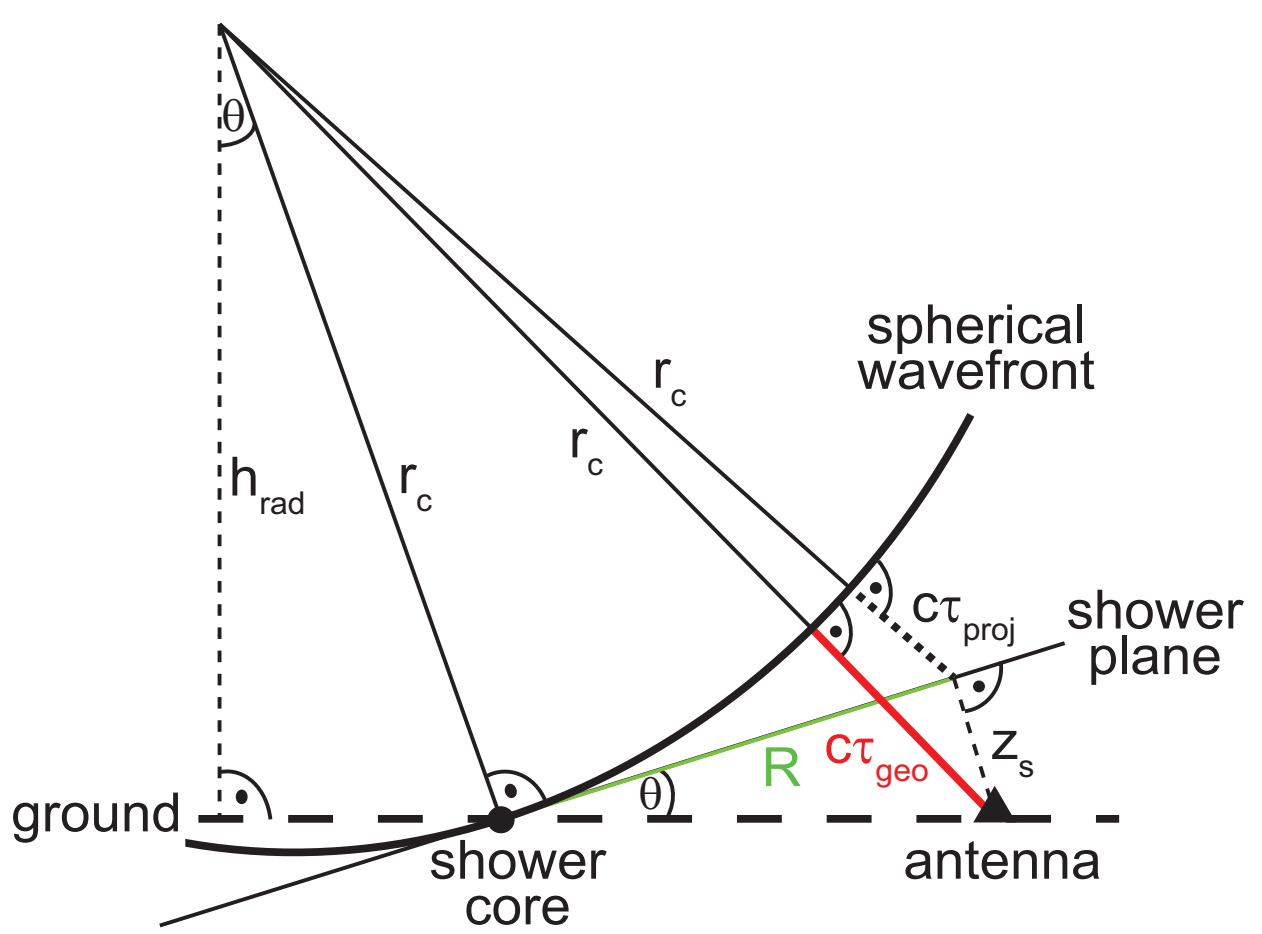

(a) spherical wavefront

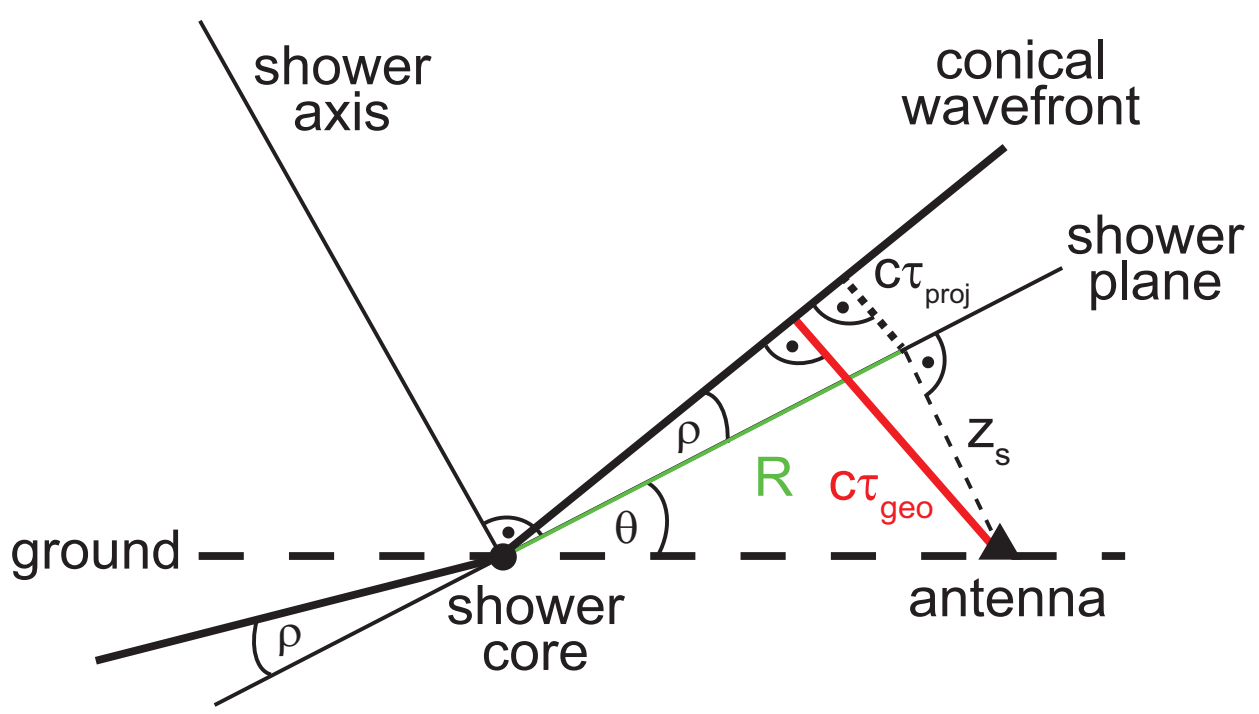

(b) conical wavefront

Figure 8.1.: Geometrical delays $\tau_{\text {geo }}\left(R, z_{s}\right)$ in dependence of the antenna position in shower coordinates $\left(R, z_{s}\right)$ and the shower geometry, for a spherical and a conical wavefront. Legend: curvature radius of the spherical wavefront $r_{\mathrm{c}}$, height of emission origin $h_{\mathrm{rad}}$, opening angle of the conical wavefront $\left(180^{\circ}-2 \rho\right)$, speed of light $c$, zenith angle $\theta$, lateral distance to the shower axis $R$, antenna height in shower coordinates $z_{\mathrm{s}}$, geometrical delay at the antenna position projected to the shower plane $\tau_{\text {proj }}(R)$. In the shower core is $\tau_{\text {geo }}(0,0)=\tau_{\text {proj }}(0)=0 \mathrm{~ns}$. 


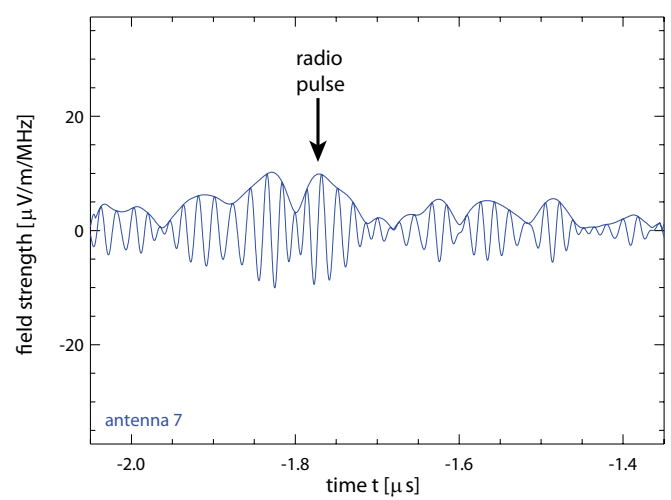

(a) $t_{\mathrm{env}-\max }=-1.774 \mu \mathrm{s}, \mathrm{SNR}=2.78$

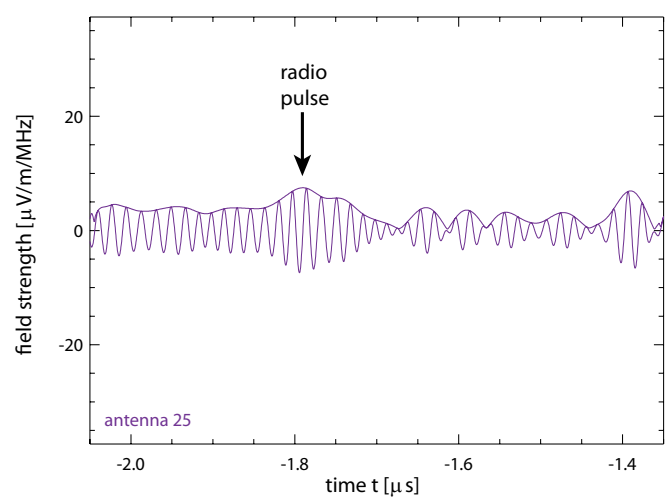

(b) $t_{\text {env }-\max }=-1.788 \mu \mathrm{s}, \mathrm{SNR}=2.01$

Figure 8.2.: Two examples for pulse arrival time measurements in individual antennas, for the event shown in figure 7.1: The radio pulse can only be identified because the time of the cross-correlation maximum, $t_{\mathrm{cc}-\max }=$ $-1.786 \mu \mathrm{s}$, is known (cf. figure 7.1). Both traces (up-sampled electric field strength and Hilbert envelope) have been shifted by the geometrical delay $\tau_{\text {geo }}$ during beamforming. Thus, the pulse is in both antennas at about the same time.

arrival time $t\left(R, z_{s}\right)$ at each antenna, since $t\left(R, z_{s}\right)$ corresponds within measurement uncertainties to the geometrical delays $\tau_{\text {geo }}\left(R, z_{s}\right)$.

In addition, the geometrical delays can be determined by beamforming. For this, a spherical wavefront with a certain curvature radius $r_{\mathrm{c}}$ is assumed in the LOPES standard analysis pipeline (see section 3.2.2). A fit maximizes the cross-correlation amplitude, by varying $r_{\mathrm{c}}$ and the arrival direction (using the KASCADE-Grande reconstructed direction as initial value). Since the cross-correlation beam implicitly depends on the pulse arrival time in each individual antenna, and so do $r_{\mathrm{c}}$ and $\tau_{\text {geo }}$. However, a beamforming analysis based on a conical wavefront is not available, yet.

\subsubsection{Pulse arrival time measurement}

For each LOPES event, the pulse arrival time $t$ in each individual antenna is measured as the time when the instantaneous amplitude is maximum (= the maximum of an Hilbert envelope). This is exactly the time when the pulse amplitude for the lateral distribution is measured (cf., section 7.1.1, figure 7.1). The maximum has been chosen because the signal-to-noise ratio of a typical measurement is very low (on average between 2 and 3, cf., chapter 6, figure 6.9). Measuring the time with a constant fraction or barycenter method might be more precise at high signal-to-noise ratios, when the pulse can clearly be distinguished from noise. However, in this case the measurement precision is anyway limited by the time calibration uncertainty of $\sim 2$ ns (cf., chapter 4 ). Thus, the time is measured by the maximum in all cases.

The electrical field strength trace of each antenna is up-sampled to $1280 \mathrm{MHz}$, and a Hilbert envelope is calculated (see appendix B). The pulse arrival time $t$ is determined with the local maximum of the envelope closest to the maximum of the cross-correlation beam (see figure 8.2). This bias on the cross-correlation beam is 
necessary to identify the radio pulse in the noise. Thus, $t$ is measured relative to the geometrical delay $\tau_{\text {geo }}$ in each antenna known from the cross-correlation beamforming: $t$ is the sum of the geometrical delay $\tau_{\text {geo }}$ and the time difference between the envelope maximum $t_{\mathrm{env}-\max }$ and the maximum of the cross-correlation beam $t_{\mathrm{cc}-\max }$ :

$$
t=\tau_{\text {geo }}+\left(t_{\text {env }-\max }-t_{\text {cc }-\max }\right)
$$

If the pulse arrival time $t$ corresponds exactly to the spherical wavefront from the cross-correlation beamforming, it equals the geometrical delay $\tau_{\text {geo }}$. Due to the measurement method, $t$ deviates from $\tau_{\text {geo }}$ typically by not more than $10-20 \mathrm{~ns}$, independently from the true time of the radio pulse. Thus, this procedure introduces a bias towards the spherical wavefront assumed in the cross-correlation beamforming. However, this cannot be avoided for LOPES, because the signal-to-noise ratio of most measurements is too low to determine the pulse arrival time without the preceding cross-correlation beamforming.

For the REAS3 simulations, the pulse arrival time has to be determined differently, since no full detector simulation is available, yet. The simulated east-west polarized component of the electrical field strength at each antenna position is filtered to the effective LOPES bandwidth $(43-74 \mathrm{MHz})$. Then, the time of the maximum is determined and a constant offset subtracted to ensure that the geometrical delay at the core is 0 . The constant offset is the same for all simulations. With a direct simulation of the radio pulse at antenna positions close to the core, the offset has been determined to $1.2 \pm 0.1 \mathrm{~ns}$. However, for events of the KASCADE selection, the mean reduced $\chi^{2}$ of a conical wavefront fit is minimized by an offset of $2.4 \mathrm{~ns}$. Thus, the constant offset has been set to $2.4 \mathrm{~ns}$ for all simulations. The difference to the $1.2 \mathrm{~ns}$ offset in the shower core is most likely caused by the change of the pulse shape with lateral distance. This results in a systematic uncertainty as discussed later.

\subsubsection{Fit of the arrival time distribution}

When a certain functional description $f(R)$ of the wavefront shape is assumed, e.g., a spherical or a conical wavefront, the geometrical delays $\tau_{\text {geo }}\left(R, z_{s}\right)$ are the distance (divided by $c$ ) from the wavefront $f(R)$ to the antenna position $\left(R, z_{s}\right)$.

Since the pulse arrival time distribution is a two-dimensional function $t\left(R, z_{s}\right)$, which is difficult to illustrate, the geometrical delays have also been calculated for a projected pulse arrival time distribution $t_{\text {proj }}(R)=t\left(R, z_{s}\right)-z_{s} / c$. This way, a one-dimensional pulse arrival time distribution $t_{\text {proj }}(R)$ is obtained, similar to the lateral distribution of pulse amplitudes $\epsilon(R)$ (cf., chapter 7). KASCADE-Grande measures the shower geometry precisely enough, such that the uncertainty of $t_{\text {proj }}$ is still dominated by the uncertainty of $t$, and not by the uncertainty of $z_{s}$. However, when projecting the pulse arrival time to the shower plane, one accepts a small mistake, as visible in figure 8.1. The geometrical delay $\tau_{\text {geo }}$ does not exactly correspond to the sum of the projected geometrical delay $\tau_{\text {proj }}$ and the time corresponding to the antenna height $z_{s} / c$. The projection is still a good approximation for a small array like LOPES (see figure 8.4). Hence, only projected pulse arrival time distributions are shown, e.g., in figure 8.3, but for the discussion of the wavefront shape and the reconstruction of $X_{\max }$ the results of the two-dimensional fits are used. 


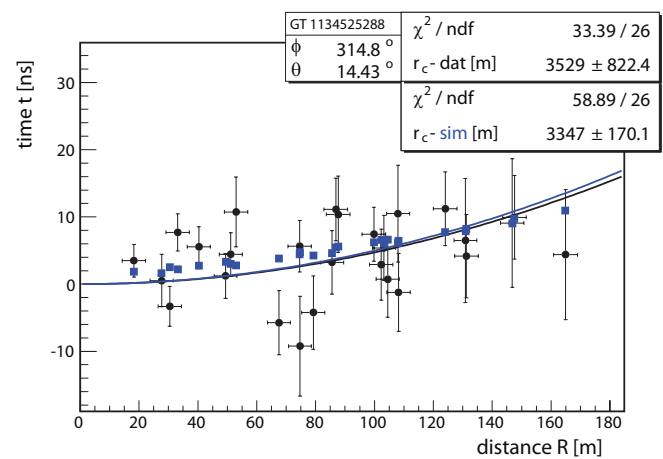

(a) LOPES $30 \mathrm{EW}$, spherical wavefront

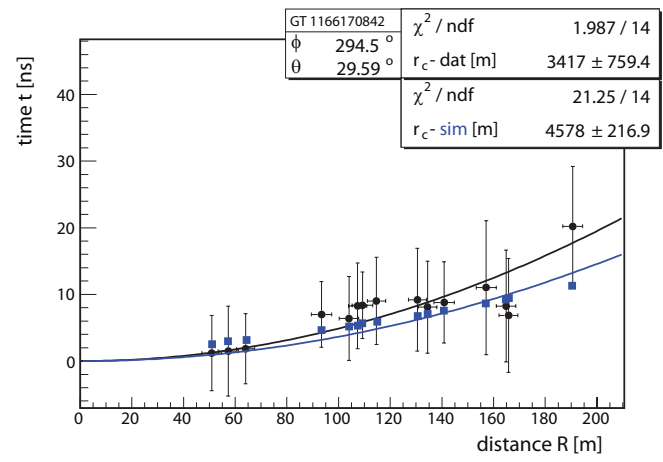

(c) LOPES Dual EW, spherical wavefront

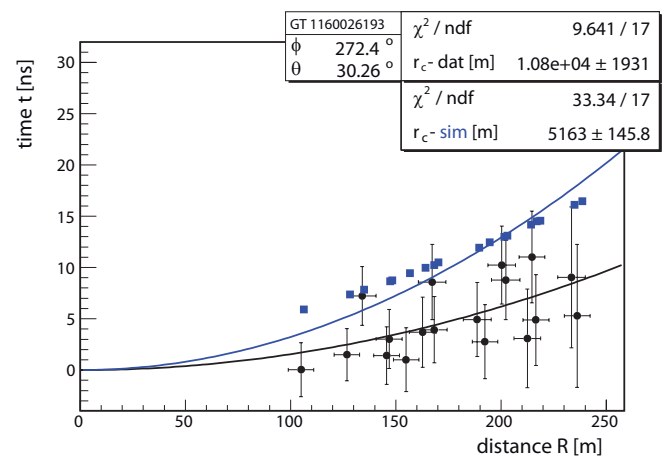

(e) LOPES 30 EW, spherical wavefront

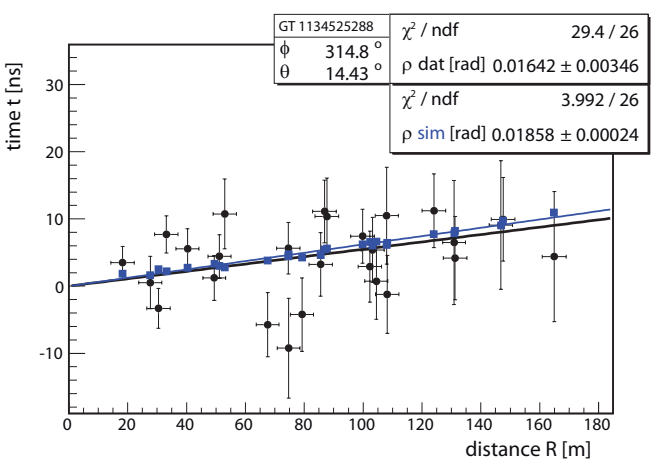

(b) LOPES $30 \mathrm{EW}$, conical wavefront

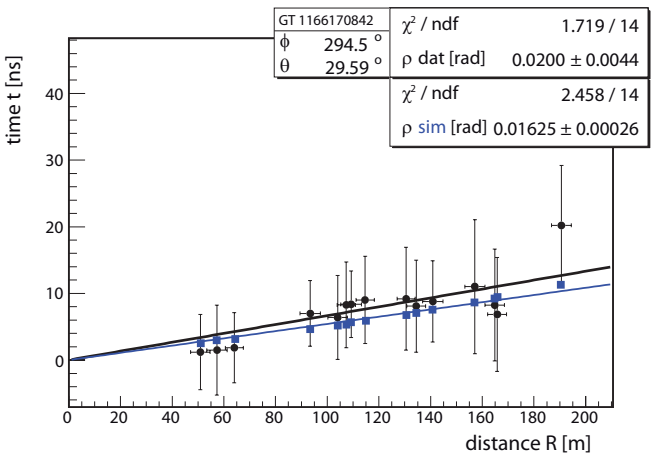

(d) LOPES Dual EW, conical wavefront

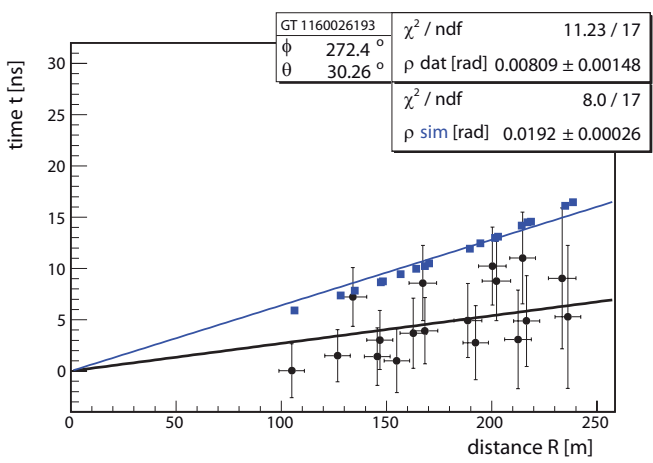

(f) LOPES $30 \mathrm{EW}$, conical wavefront

Figure 8.3.: Three examples for projected pulse arrival time distributions $t_{\text {proj }}(R)(R$ $=$ lateral distance to shower axis). Each event is shown twice, with a fit of a spherical and a conical wavefront. The LOPES measurements (east-west polarization) are shown as black circles, the corresponding REAS3 simulations for protons as primary particles as blue squares. Due to shower fluctuations, a quantitative agreement between measurements and simulations is not expected on the level of single events. A conical wavefront does not perfectly fit the simulations, but significantly better than a spherical wavefront. 

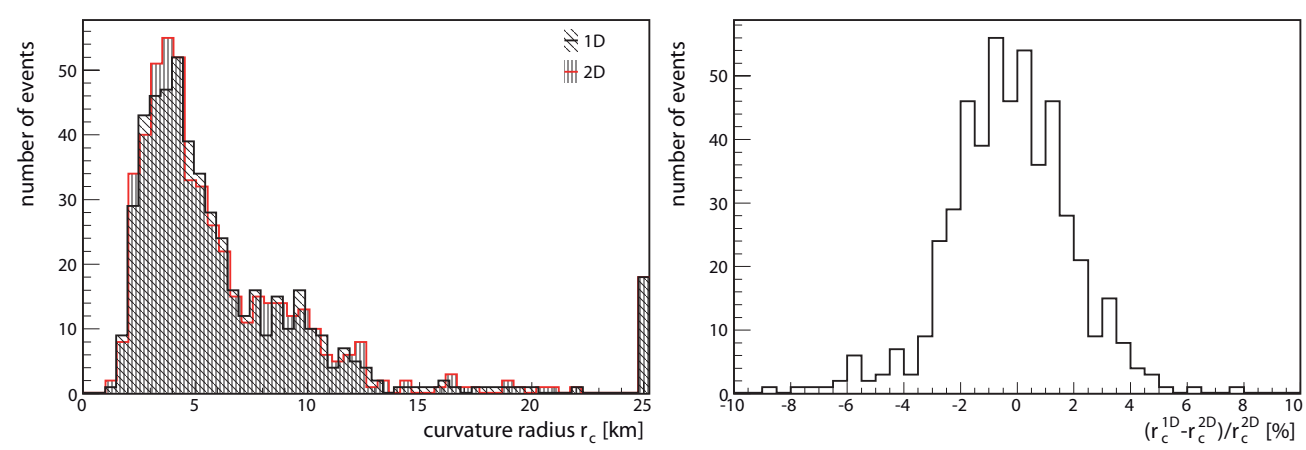

Figure 8.4.: Curvature radii $r_{c}$ determined with a fit to the two-dimensional (2D) arrival time distribution $t\left(R, z_{s}\right)$, and with a fit to the one-dimensional (1D) distribution of pulse arrival times projected to the shower plane $t_{\text {proj }}(R)$. The last bin in the left histogram contains events with $r_{c}>$ $25 \mathrm{~km}$. The deviation between the 1D and 2D fit of a few percent is negligible against many other uncertainties. This holds also true when fitting a conical wavefront.

For a spherical wavefront with a curvature radius $r_{c}$, the fit functions are:

$$
\begin{gathered}
t\left(R, z_{s}\right)=\frac{1}{c}\left(\sqrt{\left(r_{c}+z_{s}\right)^{2}+R^{2}}-r_{c}\right) \\
t_{\text {proj }}(R)=\frac{1}{c}\left(\sqrt{r_{c}^{2}+R^{2}}-r_{c}\right)
\end{gathered}
$$

with the pulse arrival time $t$ in an antenna at a lateral distance $R$ to the shower axis, the antenna height in shower coordinates $z_{s}$, and the speed of light $c$.

For further analysis, the more intuitive curvature $\kappa=1 / r_{c}$ is used instead of the curvature radius. This has practical reasons: For (almost) flat wavefronts, $r_{c}$ becomes very large and is in contrast to $\kappa$ not well defined ( $\kappa \approx 0$ for flat wavefronts). The statistical error $\Delta \kappa$ is defined such that $\Delta \kappa / \kappa=\Delta r_{c} / r_{c}$, whereby $\Delta r_{c}$ is the statistical error of the fit. This way an error estimation for flat events is not possible. Nevertheless, this is not harmful, as flat events are anyway removed by a quality cut (cf. section 8.1).

For a conical wavefront with a cone parameter $\rho(=$ angle between the cone surface and the shower plane) corresponding to an opening angle of $\left(180^{\circ}-2 \rho\right)$, the fit functions are:

$$
\begin{gathered}
t\left(R, z_{s}\right)=\frac{1}{c}(R \sin \rho+z \cos \rho) \\
t_{\text {proj }}(R)=\frac{1}{c} R \sin \rho
\end{gathered}
$$

with the pulse arrival time $t$ in an antenna at a lateral distance $R$ to the shower axis, the antenna height in shower coordinates $z_{s}$, and the speed of light $c$.

For fitting, a minimizing $\chi^{2}$ fit algorithm of the ROOT [139] software package is used. Each data point $t\left(R, z_{s}\right)$ is weighted according to its error $\Delta t$, which is the squared sum of the error due to noise (cf., section 6.5) and the calibration uncertainty of $2 \mathrm{~ns}$ (cf., chapter 4 ). Since the geometrical delay $\tau_{g e o}(R, z)$ is per definition 0 at 


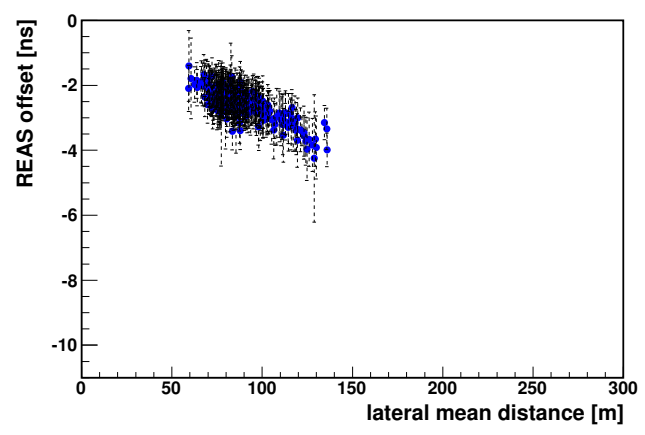

(a) simulations for KASCADE events

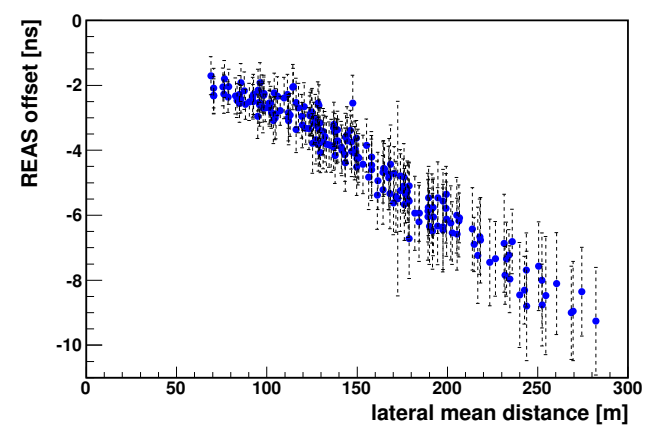

(b) simulations for Grande events

Figure 8.5.: Dependence of a variable time offset in the conical fit on the mean lateral distance of the antennas. Each point corresponds to one event simulated with REAS3 for proton primaries. The error bars are the statistical uncertainties of the fit. The corresponding plots for REAS3 iron simulations are not shown, since they are very similar.

the shower core $(R, z)=(0,0)$, no time offset is considered in the fit. For testing purposes, a variable time offset has been included in the fit, and for LOPES events the results are consistent with a time offset of 0 . Moreover, including a time offset causes the fit to fail for about $20 \%$ of the events, without significantly improving the fit results of the remaining events.

However, for the REAS3 simulations there is a time offset as explained in section 8.2.1. The offset is expected to be constant, since it ought to depend only on the method of how to measure the pulse arrival time (time of pulse maximum), which is the same for each simulation. For the conical wavefront, the offset has been investigated in detail: If a variable offset is allowed, it depends on the mean lateral distance of the simulated antennas positions (see figure 8.5) - contrary to the naive expectation. This can be explained by the change of the pulse shape with lateral distance: the maximum time probably shifts with respect to the start time of the pulse dependent on the pulse shape. However, trying to correct for this by a variable time offset in the fit causes two problems: First, the statistical error of $\rho$ increases. Second, $\rho$ depends on the lateral mean distance, which is not the case if the offset is fixed. Therefore, the offset has been fixed to the $2.4 \mathrm{~ns}$ already mentioned above. A change in the offset of 1 ns leads to a change in $\rho$ of $\approx 0.0029 \mathrm{rad} \approx 0.17^{\circ}$, which gives rise to a corresponding systematic uncertainty of $\rho$. Further investigations will be necessary to understand the dependence of the offset on the lateral distance and the method of pulse arrival time measurements in detail, and thus to decrease this systematic uncertainty.

\subsubsection{Comparison of curvature reconstruction methods}

For the LOPES measurements, the curvatures reconstructed by cross-correlation beamforming $\kappa_{\mathrm{cc}}$ and the pulse arrival time distributions $\kappa_{\text {atd }}$ are compared with each other. In the ideal case, $\kappa_{\mathrm{cc}}$ ought to be equal to $\kappa_{\text {atd }}$. Deviations from $\kappa_{\mathrm{cc}}=\kappa_{\text {atd }}$ can 

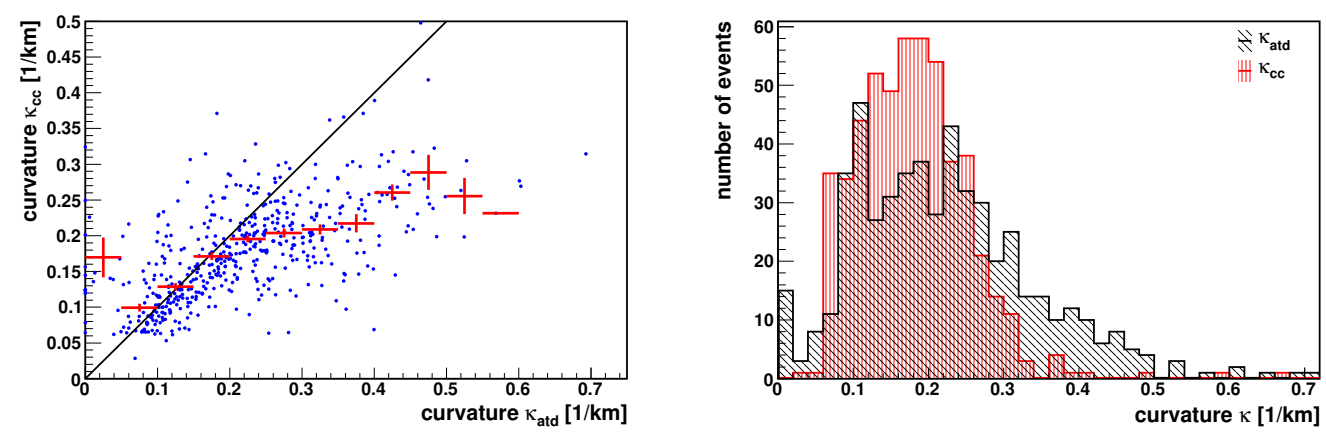

Figure 8.6.: Comparison between the curvatures reconstructed by cross-correlation beam-forming $\kappa_{\mathrm{cc}}$ and the pulse arrival time distribution $\kappa_{\mathrm{atd}}$.

indicate systematic effects in the reconstruction procedure, or that the assumption of a spherical wavefront is insufficient.

The comparison shows that $\kappa_{\text {cc }}$ equals $\kappa_{\text {atd }}$ for curvatures in an intermediate range of $\sim 0.1-0.2 \mathrm{~km}^{-1}$ (figure 8.6). The deviation for low curvatures, i.e, for flat events, is not surprising. Due to the algorithm used for cross-correlation beamforming there is a lower limit for flat events of $\kappa_{\mathrm{cc}} \gtrsim 0.05 \mathrm{~km}^{-1}$, which does not exist for $\kappa_{\text {atd }}$. The deviation for large curvatures is more meaningful. Neither the origin of the deviation is clear, nor which of the two values for $\kappa$ is more accurate. An interferometric method, like cross-correlation beamforming, is thought to be more precise than a non-interferometric method, like fitting the pulse arrival time distribution. However, cross-correlation beamforming is not necessarily more accurate, since it has an unsolved systematic problem: like any interferometer, LOPES shows some side lobes. This means that there exist several local maxima of the cross-correlation amplitude corresponding to different $\kappa_{\mathrm{cc}}$. This causes a large systematic uncertainty on $\kappa_{\mathrm{cc}}$, and prevents an estimation of the total uncertainty $\Delta \kappa_{\mathrm{cc}}$.

A comparison of a reconstruction with beamforming and pulse arrival time distributions has not been performed for the conical wavefront assumption, since no implementation of a conical cross-correlation beamforming is available, yet. Neither has the comparison been performed for simulations, because it would require a full simulation of detector effects. Such an end-to-end simulation for LOPES is still under development [134]. This functionality is already implemented for AERA in the Offline software package. However, Offline still misses an interferometric pipeline which is planned for the near future. Then it will be possible to study the wavefront shape with AERA events and simultaneously with REAS3 simulations, and to compare the beamforming with the arrival time distribution analysis.

The lack of beamforming results for simulations and the conical wavefront is not a major drawback. For the spherical wavefront, LOPES curvatures show the same qualitative dependencies on shower parameters, and experience only a quantitative difference in $\kappa$, as already seen in figure 8.6. Thus, all qualitative features of the wavefront ought to be revealed by only studying the pulse arrival time distributions. For this reason, the following sections focus on the results of pulse arrival time distributions. 

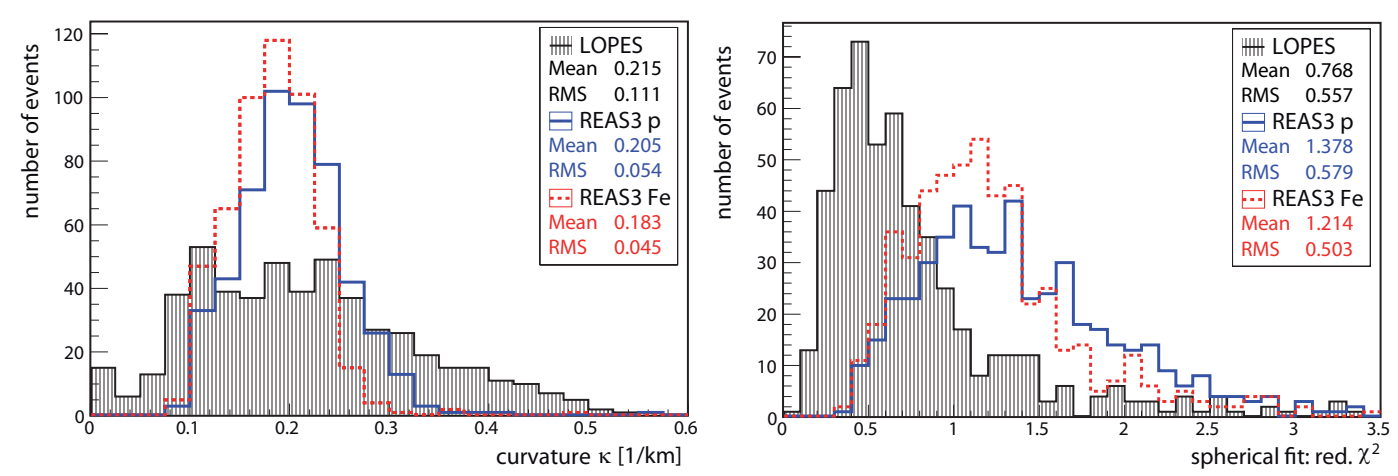

Figure 8.7.: Curvature $\kappa$ of LOPES events and REAS3 simulations for proton and iron primaries, and the reduced $\chi^{2}$ of the fits.

\subsection{Investigation of the wavefront shape}

The shape of the wavefront is investigated for LOPES events and REAS3 simulations with pulse arrival time distributions. For this, the fit results for a spherical wavefront and a conical wavefront are evaluated. To investigate which assumption approximates measurements and simulations better, the reduced $\chi^{2}$ of the fits is studied, as well as dependencies of the curvature $\kappa$ and the cone parameter $\rho$ on other shower parameters. For the simulations, mainly the results of REAS3 proton simulations are shown. The results of the REAS3 iron simulations are qualitatively similar. They cover a different range of $\kappa$ respectively $\rho$, but feature the same dependencies on other shower parameters.

\subsubsection{Spherical wavefront}

The curvature of both LOPES and REAS3 pulse arrival times is typically a few $0.1 \mathrm{~km}^{-1}$ (see figure 8.7), which corresponds to a point source with a height of a few $\mathrm{km}$. This is reasonable since the height of the shower maximum is in the same order. Also the reduced $\chi^{2}$ of LOPES measurements is compatible with a spherical curvature. It is even significantly below 1 , which means that either the data are biased, or that the errors $\Delta t$ are overestimated. A bias towards a spherical wavefront is expected, since the pulse arrival time measurement is biased by the cross-correlation beam, for which a spherical wave front has already been assumed. An overestimation of the errors cannot be excluded completely. The noise influence on pulse arrival time measurements in individual antennas has been investigated carefully, but a possible correlation of the noise influence in different antennas has not been studied, yet.

For the simulations, there is no bias towards a spherical wavefront expected, because the arrival time distributions do not rely on a preceding beamforming analysis. The absolute value of the reduced $\chi^{2}$ is of limited interest, since there exists no measurement error for simulations. But the relative comparison of the $\chi^{2}$ between the spherical and the conical fit is meaningful. In the next section we will see that the $\chi^{2}$ of the conical fit is generally smaller for the simulations.

A conclusive test is whether the curvature of the measured and simulated events depends on other shower parameters in the expected way (see figures 8.8 and 8.9). 

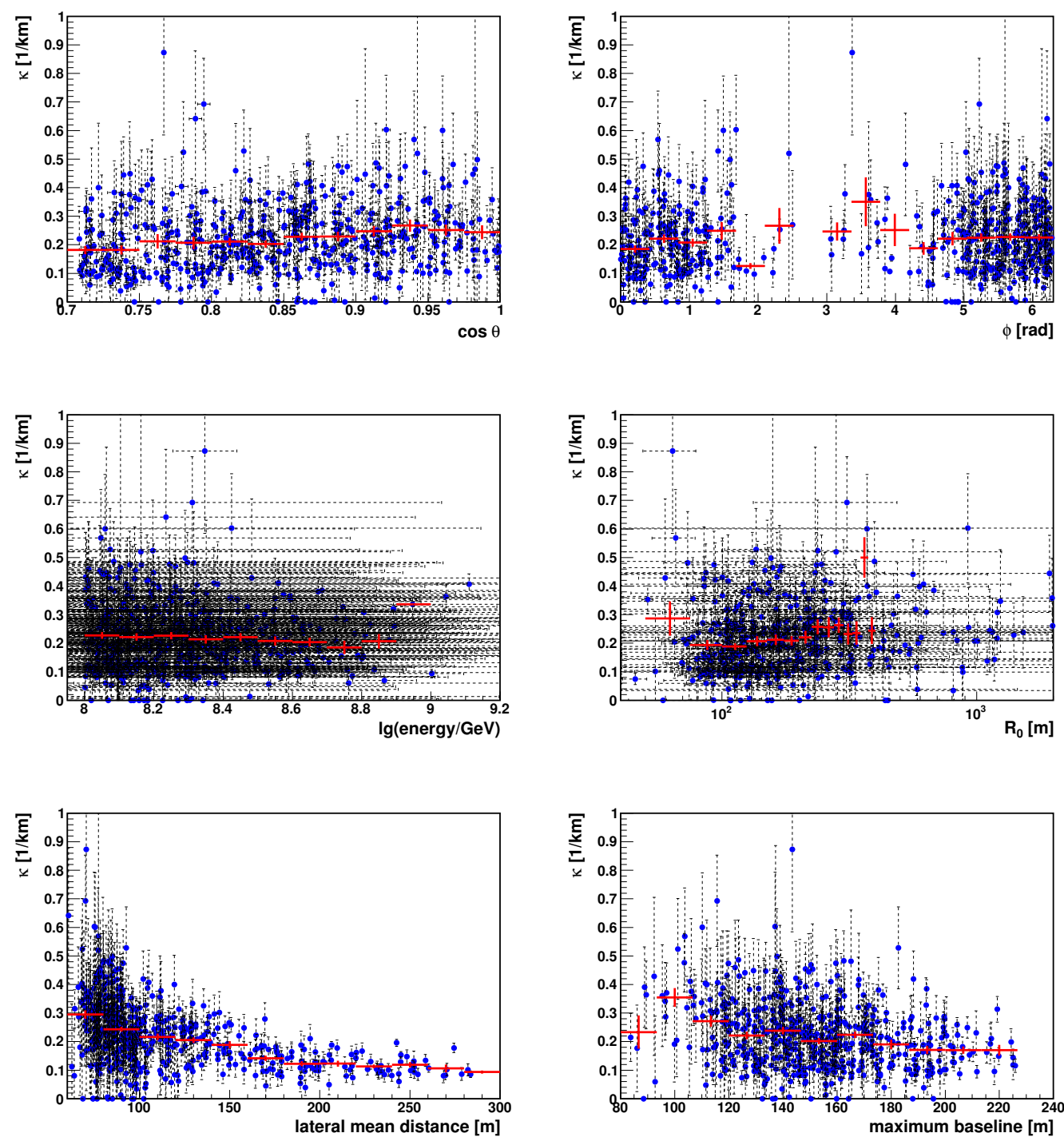

Figure 8.8.: Dependencies of the wavefront curvature $\kappa$ on other shower parameters, for LOPES events. From left to right, from top to bottom: the shower zenith $\theta$, the azimuth $\phi$, the primary energy $E$ reconstructed by KASCADE-Grande, the slope parameter of the lateral distribution $R_{0}$, the mean lateral distance $R_{\text {mean }}$ of the antennas contributing to an event, and the maximum baseline $\left(=R_{\max }-R_{\min }\right)$. A correlation of $\kappa$ with the zenith angle is expected. For the azimuth and the energy, only a marginal correlation is expected which is beyond the measurement precision of LOPES. Furthermore, a correlation between $R_{0}$ and $\kappa$ is expected since both parameters depend on the mean lateral distance and are thought to be mass sensitive. In the two bottom plots, no correlation is expected, if a spherical wavefront is a good approximation for the measured pulse arrival time distributions. 

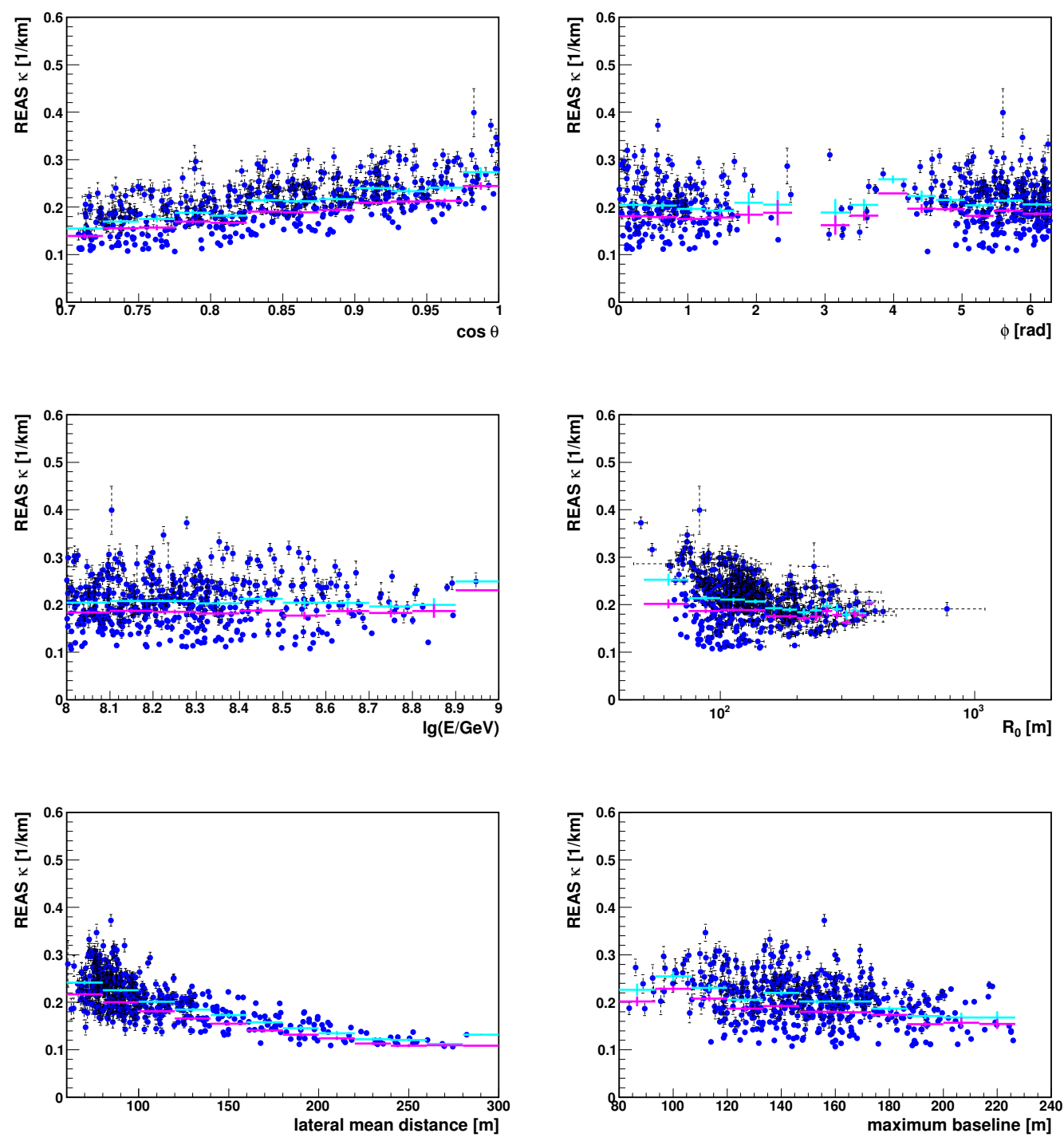

Figure 8.9.: Dependencies of the wavefront curvature $\kappa$ on other shower parameters, for REAS3 simulations assuming a proton as primary particle. See caption of figure 8.8 for a description of the plots and the interpretation. The REAS3 simulations experience generally the same dependencies on shower parameters as the measured LOPES events. However, the spread of $\kappa$ is smaller than for the LOPES data indicating the large measurement uncertainty. The light blue profile is for the plotted points, i.e., the simulations for proton primaries. The systematically lower pink profile is for iron primaries. 

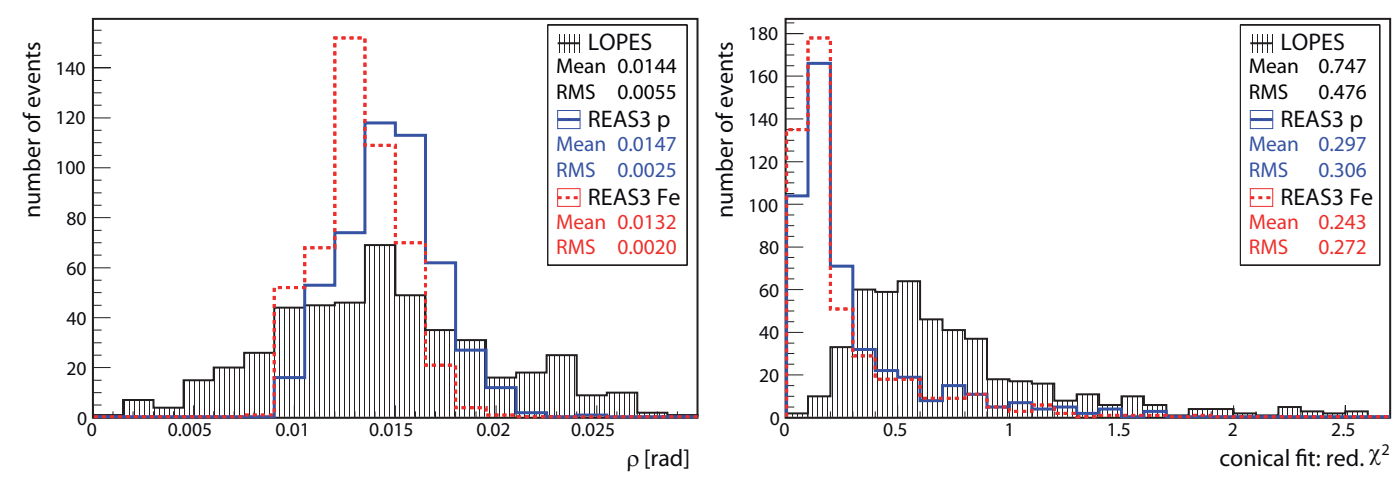

Figure 8.10.: Cone parameter $\rho$ of LOPES events and REAS3 simulations for proton and iron primaries, and the reduced $\chi^{2}$ of the fits.

As expected, the curvature depends on the zenith angle, since the distance from ground to the shower maximum depends on the zenith angle. Other dependencies on the shower geometry, e.g., the azimuth, are expected to be very weak, since they are second order effects. Indeed, no significant correlation is observed. The mean curvature ought to increase with the primary energy, since the mean $X_{\max }$ increases with energy. However, the effect is relatively small in the limited energy range covered by LOPES, and no significant correlation is observed.

Moreover, the radio wavefront curvature $\kappa$ ought to correlate with other radio parameters, especially with the slope of the lateral distribution of pulse amplitudes. Since iron showers have on average a larger slope parameter $R_{0}$ than proton showers (cf., section 7.4), $\kappa$ ought to decrease with increasing $R_{0}$ : A shower which has a flat arrival time distribution is expected to have also a flat lateral distribution of amplitudes. However, such a correlation cannot be seen - neither in the LOPES events nor in the simulations. This is a further hint that a spherical wavefront is an insufficient approximation to the real wavefront.

An even stronger hint is the clear correlation between $\kappa$ and the mean lateral distance $R_{\text {mean }}$. This means that the curvature depends on the lateral distance where it is measured, which is not expected for a spherical wavefront, since the curvature of a sphere is a constant. This indicates that the wavefront should be approximated more accurately by another function. The weak dependence of $\kappa$ on the maximum baseline $\left(R_{\max }-R_{\min }\right)$ is probably caused by the dependence of $\kappa$ on the mean lateral distance $R_{\text {mean }}$. Events of the Grande selection with a core position outside of the LOPES array have a large maximum baseline and a large $R_{\text {mean }}$, while events with a core inside of the LOPES array automatically have a smaller maximum baseline and $R_{\text {mean }}$.

\subsubsection{Conical wavefront}

A conical wavefront is a better approximation to the measured and simulated arrival time distributions than a spherical wavefront. For the simulations, this can be clearly seen from the histogram of reduced $\chi^{2}$, which is in general much smaller than for the spherical wavefront (see figure 8.10). For the measured LOPES events, the reduced $\chi^{2}$ for the conical wavefront is about the same as for the spherical wavefront, although 
the LOPES pulse arrival time distributions are biased towards a spherical wavefront by the preceding cross-correlation beamforming.

The dependencies of the cone parameter $\rho$ on other shower parameters support the hypothesis that a conical wavefront is a better approximation (see figures 8.11 and 8.12). The expected dependencies for $\rho$ are the same as for the curvature $\kappa$ of the spherical wavefront. As expected, a clear correlation with the zenith $\theta$ is observed, which is the main motivation, why $\rho$ is not directly used to estimate the primary mass, but instead $X_{\max }$ (see next section). No correlation is observed between $\rho$ and the azimuth $\phi$, as expected, too. Due to the energy dependency of $X_{\max }$ also a dependency of $\rho$ on the energy is expected. Like for the curvature, the expected effect is relatively small in the limited energy range covered by LOPES, and thus no significant correlation is observed.

For the simulations, $\rho$ is clearly correlated with the slope parameter $R_{0}$ of the lateral distributions of amplitudes: Showers with a flat amplitude lateral distribution have also a flat pulse arrival time distribution. This is expected, since both radio parameters, $\rho$ and $R_{0}$, are sensitive to the shower maximum. However, the correlation is not observed in the LOPES measurements. This indicates that the measurement precision of $\rho$ or $R_{0}$ or both of them is insufficient for a per-event mass reconstruction.

To investigate whether the problem is the uncertainty of $\rho$ or $R_{0}$, a cross-comparison of $\rho$ and $R_{0}$ for LOPES events and the corresponding REAS3 simulations for proton primaries is performed (figure 8.13). The comparision gives a hint that $R_{0}$ can be measured more precisely than $\rho$, since there is a correlation in only one case $\left(R_{0}^{\text {LOPES }}\right.$ versus $\left.\rho^{\text {REAS }}\right)$. However, one has to keep in mind that this analysis could be biased since both $\rho$ and $R_{0}$ depend on the zenith angle. It might also be that the uncertainty of $\rho$ is increased due to the bias towards a spherical wavefront by the preceding cross-correlation beamforming. This has to be checked by the implementation of a beamforming procedure based on a conical wavefront. Other reasons for the large uncertainty of $\rho$ are probably the small lateral extension of LOPES and the high ambient noise level causing large uncertainties in the pulse arrival time measurements.

For the LOPES measurements, the cone parameter $\rho$ does not depend on the mean lateral distance $R_{\text {mean }}$ and on the maximum baseline - as expected, if the wavefront is described correctly by a cone. The cone parameter of each individual shower ought to be universal and independent of the lateral distance. Besides the smaller reduced $\chi^{2}$ in the simulations, this is the second strong hint that a conical wavefront approximates the real wavefront better than a spherical wavefront. However, in the simulations, there is a weak dependency between $\rho$ and $R_{\text {mean }}$ observed. There are three possible explanations for this dependency. First, it could be related to the problem of determining the time offset for simulated pulse arrival times. Second, the dependency between $\rho$ and $R_{\text {mean }}$ might indicate that the conical wavefront is still an approximation and not a perfect description of the real wavefront. This matches the impression from the pulse arrival time distribution of individual events (see figure 8.3). Third, it might indicate that the conical wavefront cannot be reconstructed accurately if the shower core - and thus the apex of the cone - is outside of the antenna array, because exactly those showers have a large maximum baseline and $R_{\text {mean }}$. Therefore, only showers with a core contained in the LOPES array are used to reconstruct the shower maximum. 

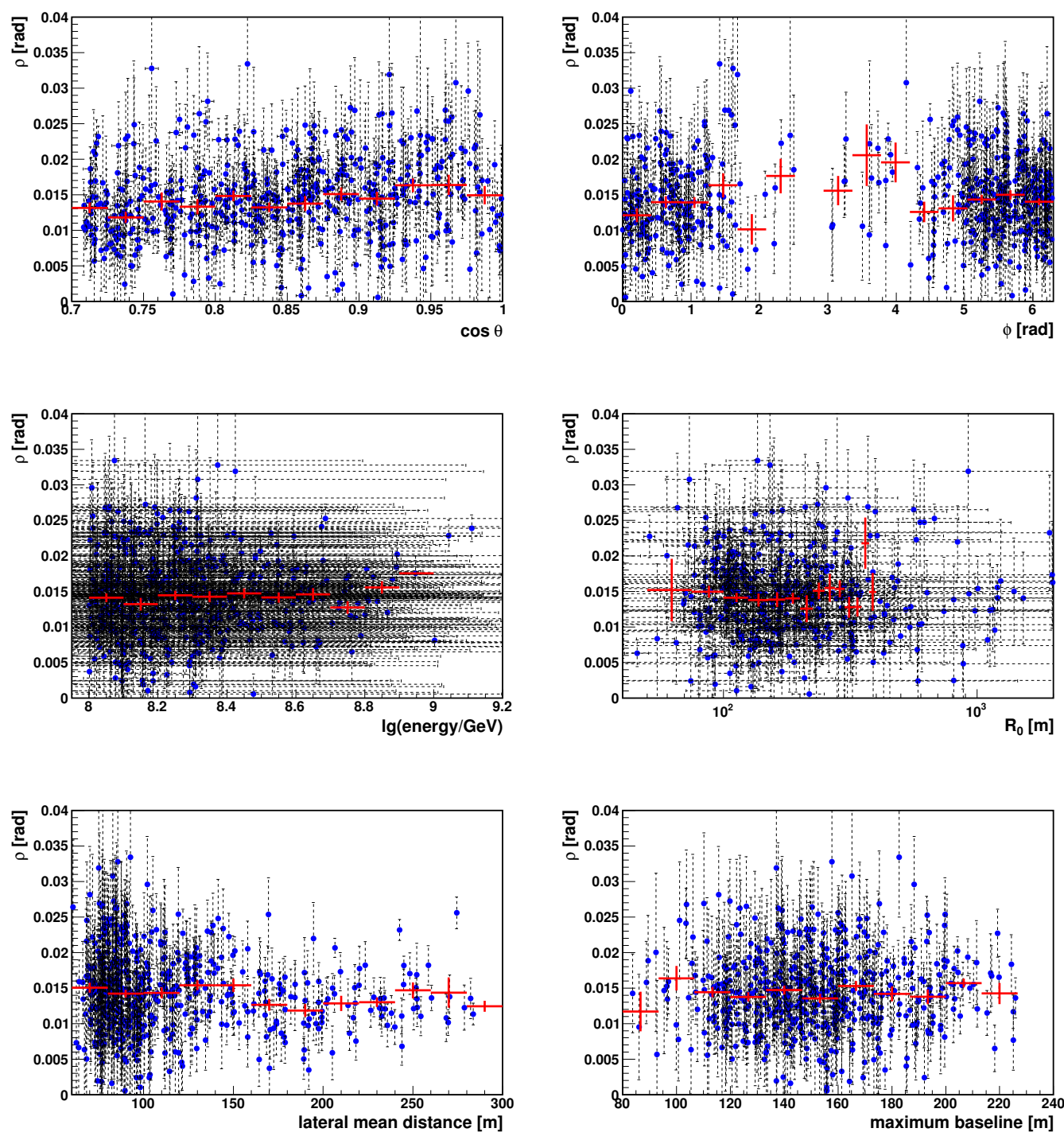

Figure 8.11.: Dependencies of the cone parameter $\rho$ on other shower parameters, for LOPES events. From left to right, from top to bottom: the shower zenith $\theta$, the azimuth $\phi$, the primary energy $E$ reconstructed by KASCADE-Grande, the slope parameter of the lateral distribution $R_{0}$, the mean lateral distance $R_{\text {mean }}$ of the antennas contributing to an event, and the maximum baseline $\left(=R_{\max }-R_{\min }\right)$. A correlation of $\rho$ with the zenith angle is expected. For the azimuth and the energy, only a marginal correlation is expected which is beyond the measurement precision of LOPES. Furthermore, a correlation between $R_{0}$ and $\rho$ is expected, since both parameters are thought to depend on $X_{\max }$. In the two bottom plots, no correlation is expected, if a conical wavefront is a good approximation for the measured pulse arrival time distributions. 

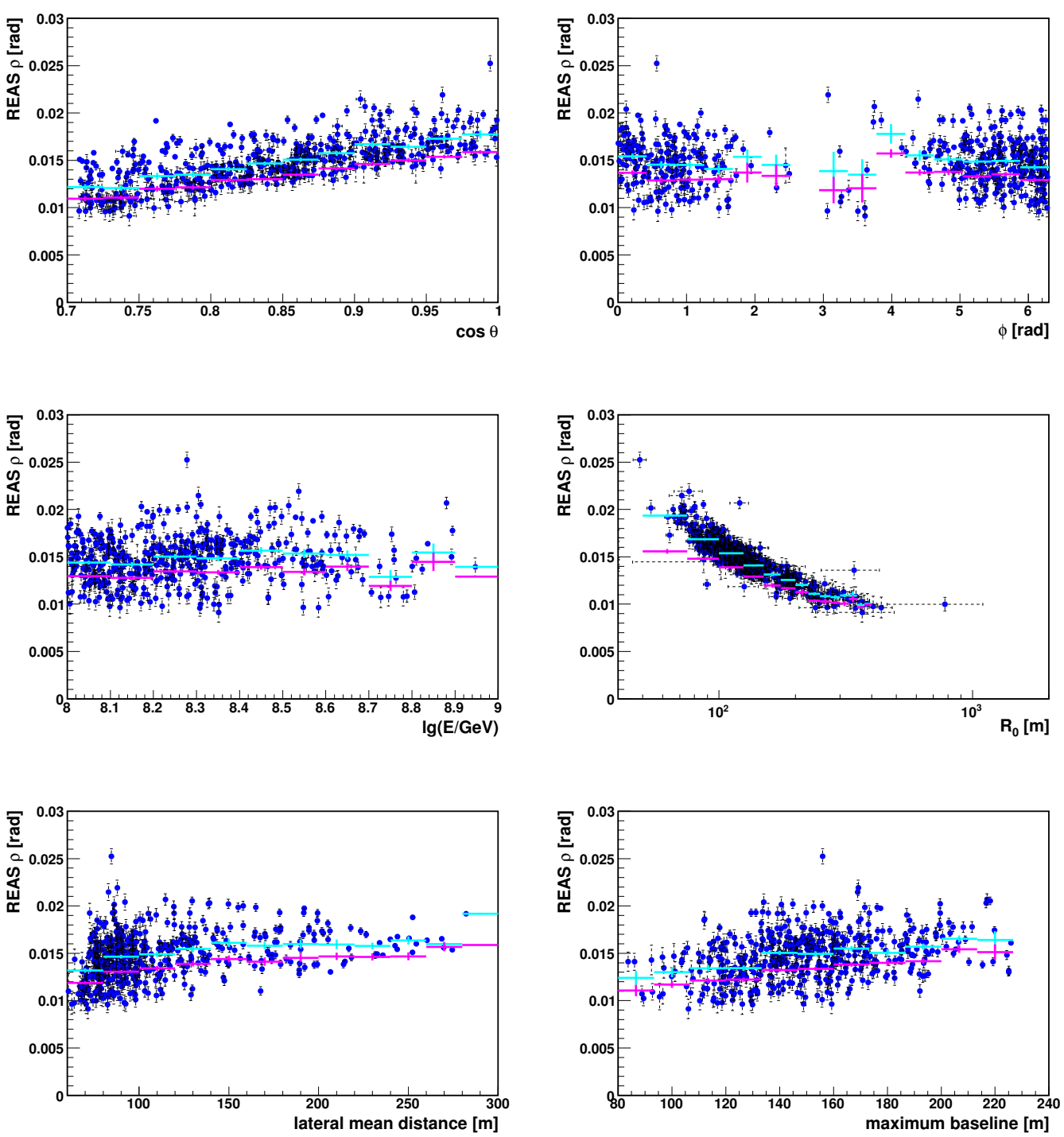

Figure 8.12.: Dependencies of the cone parameter $\rho$ on other shower parameters, for REAS3 simulations assuming a proton as primary particle. See caption of figure 8.11 for a description of the plots and the interpretation. The spread of $\rho$ is smaller for the simulations than for the LOPES data indicating the large measurement uncertainty. The REAS3 simulations for iron primaries are not shown since they are qualitatively similar. The correlation of $\rho$ with the mean lateral distance $R_{\text {mean }}$ and the maximum baseline is observed only for events of the Grande selection, which have generally a larger $R_{\text {mean }}$ and maximum baseline than the events of the KASCADE selection. The light blue profile is for the plotted points, i.e., the simulations for proton primaries. The systematically lower pink profile is for iron primaries. 

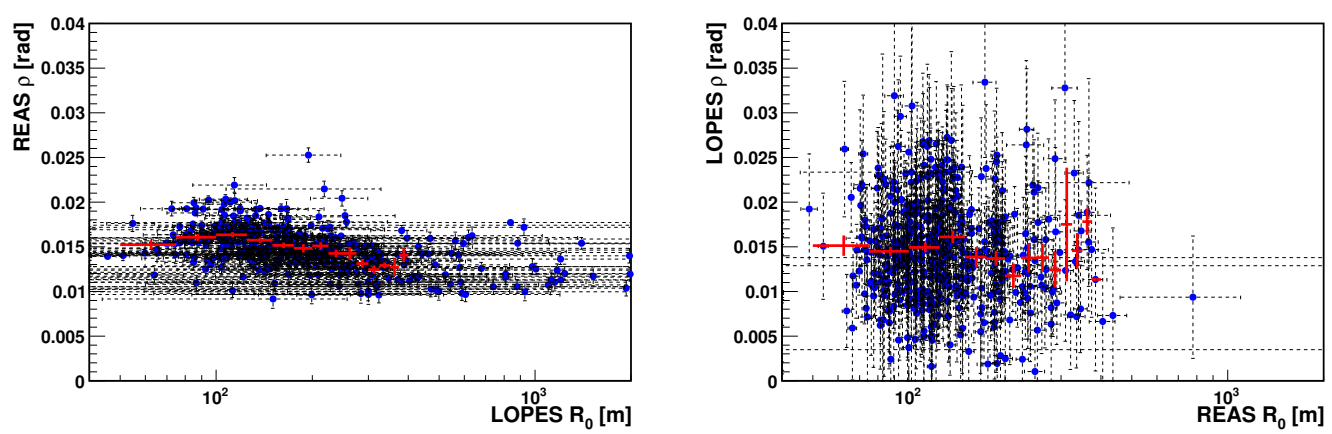

Figure 8.13.: Cross-comparison of $\rho$ and $R_{0}$ for LOPES events and the corresponding REAS3 simulations for proton primaries. The comparision gives a hint that $R_{0}$ can be measured more precisely than $\rho$, since $R_{0}^{\mathrm{LOPES}}$ is correlated with $\rho^{\text {REAS }}$, but $\rho^{\text {LOPES }}$ is not correlated with $R_{0}^{\text {REAS }}$.
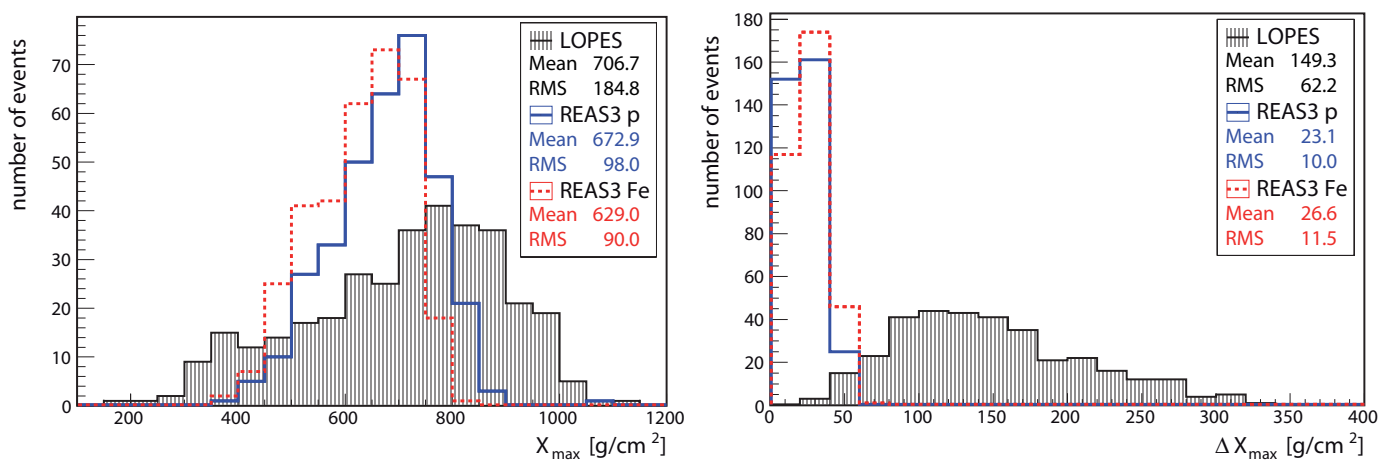

Figure 8.14.: Left: $X_{\max }$ reconstructed by fitting a spherical wavefront, for LOPES events and for the corresponding REAS3 simulations for proton and iron primaries. Right: statistical error $\Delta X_{\max }$.
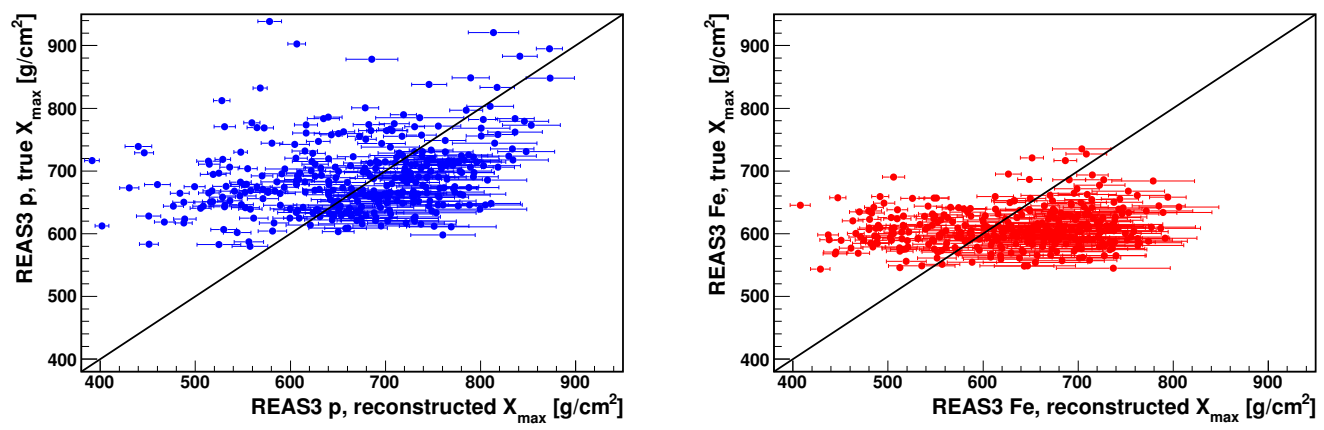

Figure 8.15.: Scatter plots of $X_{\max }$ reconstructed by fitting a spherical wavefront versus the true $X_{\max }$ of REAS3 simulations for proton and iron primaries (left and right). The large scatter comes partially due to the dependence of $\kappa$ on the lateral mean distance. 


\subsection{Determination of $X_{\max }$}

The atmospheric depth of the shower maximum $X_{\max }$ depends only marginally on the shower geometry and is sensitive to the mass of the primary particle. The most precise reconstructions of primary masses come from observation technologies which can directly probe $X_{\max }$, like fluorescence and Cherenkov light detectors. Also radio measurements ought to have a direct access to $X_{\max }$, since the radio emission reflects the longitudinal shower development. However, this has not been proven experimentally, yet. The aim of the present studies is the qualitative proof that a reconstruction of $X_{\max }$ is possible by measuring the radio wavefront with digital antenna arrays. However, due to the high ambient noise level and the small lateral extension, a precise reconstruction of $X_{\max }$ is beyond the capabilities of LOPES.

$X_{\max }$ can be reconstructed from the wavefront shape determined with a fit to pulse arrival time distributions. Although, the spherical fit does not approximate the real wavefront as well as the conical fit, it has the advantage of a simple geometric interpretation: The curvature $\kappa$ of a spherical wavefront corresponds to a point source located in the shower maximum. Since the wavefront is not spherical, the reconstruction of $X_{\max }$ with the curvature is expected to be neither precise nor accurate. Nevertheless, it can be shown that the reconstructed $X_{\max }$ has the order of magnitude expected for nuclear cosmic rays.

The procedure how $X_{\max }$ can be reconstructed, first, with the curvature $\kappa$, and second, with the cone parameter $\rho$ is explained in the following. Furthermore, the mass sensitivity of the reconstructed $X_{\max }$ is investigated for LOPES events and for REAS3 simulations.

\subsection{1. $X_{\max }$ reconstruction with curvature method}

$X_{\max }$ can be estimated from the curvature $\kappa$ and the arrival direction of the air shower, assuming that the shower maximum is approximately the origin of the radio pulse. In any case the $X_{\max }$ reconstruction with the curvature method is not expected to be very accurate. Thus, only a simple atmospheric model has been applied, namely the barometric height formula for an atmosphere at constant temperature $T$ :

$$
X_{\text {max }}=c_{s c} \cdot X_{\text {ground }} \cdot \exp -\frac{h_{\text {rad }}}{h_{0}}
$$

with the zenith $\theta$, the total atmospheric depth $X_{\text {ground }} \approx 1022 \mathrm{~g} / \mathrm{cm}^{2}$ at ground level [140], and the height of the radio emission source $h_{\mathrm{rad}}=\cos \theta / \kappa$. The height constant $h_{0}=R T / M g$ includes the gas constant $R=8.3145 \mathrm{~J} / \mathrm{mol} / \mathrm{K}$, the air temperature $T$, the molar mass $M \approx 0.029 \mathrm{~kg} / \mathrm{mol}$, and the gravitational acceleration constant $g=$ $9.81 \mathrm{~m} / \mathrm{s}^{2}$. For this analysis, $h_{0}$ is set to $7 \mathrm{~km}$, which corresponds to a temperature $T \approx 240 \mathrm{~K}$. A change in $T$ of $10 \mathrm{~K}$ changes $h_{0}$ by about $0.3 \mathrm{~km}$ and $X_{\max }$ by $15-$ $20 \mathrm{~g} / \mathrm{cm}^{2}$. The leading scaling factor $c_{s c}$ is set to 1.25 to reproduce the mean $X_{\max }$ of the REAS3 simulations. One could argue that a factor of $\cos ^{-1} \theta$ should enter in $c_{s c}$, since inclined showers have to trespass a bigger atmospheric density to reach the same height above ground as vertical showers. However, such a factor of $\cos ^{-1} \theta$ was found to increase the zenith dependency of the reconstructed $X_{\max }$, and consequently is omitted. 
The statistical error $\Delta X_{\max }$ is estimated with Gaussian error propagation, assuming that all contributions to the statistical error can be neglected against the large uncertainty of the curvature $\Delta \kappa$ :

$$
\frac{\Delta X_{\max }}{X_{\max }}=\frac{\cos \theta}{h_{0}} \frac{\Delta \kappa}{\kappa}
$$

The mean statistical error for the selected LOPES events is $\Delta X_{\max } \approx 150 \mathrm{~g} / \mathrm{cm}^{2}$, which is too large for a per-event mass estimation. Furthermore, the absolute scale of $X_{\max }$ can easily be shifted by $100 \mathrm{~g} / \mathrm{cm}^{2}$ or more by tuning $c_{s c}$ and $T$. Nevertheless, the average reconstructed $X_{\max }$ is consistent with the expectation for nuclear cosmic rays (see figure 8.14). This shows that a rough estimation of the average primary mass is in principle feasible with the curvature method, although the wavefront is not spherical. However, the reconstructed $X_{\max }$ does not reproduce the distributions for proton and iron primaries well.

Scatter plots of the true versus the reconstructed $X_{\max }$ of the REAS3 simulations (figure 8.15) reveal the large systematic uncertainty of the curvature method for $X_{\max }$ reconstruction. The large uncertainty of the reconstructed $X_{\max }$ is partially caused by the dependence of $\kappa$ on the lateral mean distance $R_{\text {mean }}$. Even if only events of the KASCADE selection, i.e., events with about the same $R_{\text {mean }}$, are considered, the $X_{\max }$ uncertainty is still larger than for the cone method presented in the following. Consequently, the curvature method is not suited for a reconstruction of the primary composition. Thus, it is surprising that in reference [107] a $X_{\max }$ resolution of about $20 \mathrm{~g} / \mathrm{cm}^{2}$ could be achieved for REAS2 simulations assuming a spherical wavefront. Perhaps the wavefront was indeed spherical in REAS2. Moreover, either the parametrization of the geometrical delays used in reference [107] or any regularities in the set of simulations might have caused the remarkable good $X_{\max }$ resolution.

\subsection{2. $X_{\max }$ reconstruction with cone method}

Since the wavefront is approximately conical, reconstructing $X_{\max }$ with the cone parameter $\rho$ is expected to by more precise and accurate than with the curvature method. A reconstruction is possible, because $X_{\max }$ is correlated with $\rho$ (see figure 8.16 , left). Since the cone parameter $\rho$ depends on the zenith angle $\theta$, it is corrected by:

$$
\rho_{\text {cor }}=\rho \cos ^{-3 / 2} \theta
$$

The factor of $\cos ^{-3 / 2} \theta$ was found to remove the zenith dependency of $\rho_{\text {cor }}$ for the simulations. For the LOPES events, rather a correction by $\cos ^{-1} \theta$ would remove the zenith dependency. This is the same correction factor which is applied to the curvature $\kappa$ for simple geometrical reasons. However, the LOPES measurements are biased towards a spherical wavefront and generally have larger uncertainties than the simulations. Thus, also for the LOPES events $\rho$ is corrected by $\cos ^{-3 / 2} \theta$. It has not been investigated why a correction factor of $\cos ^{-3 / 2} \theta$ fits better for the simulations than a factor of $\cos ^{-1} \theta$. In the present studies, the principle feasibility of the cone method for $X_{\max }$ reconstruction is in the focus, which is thought not to depend on this detail. 

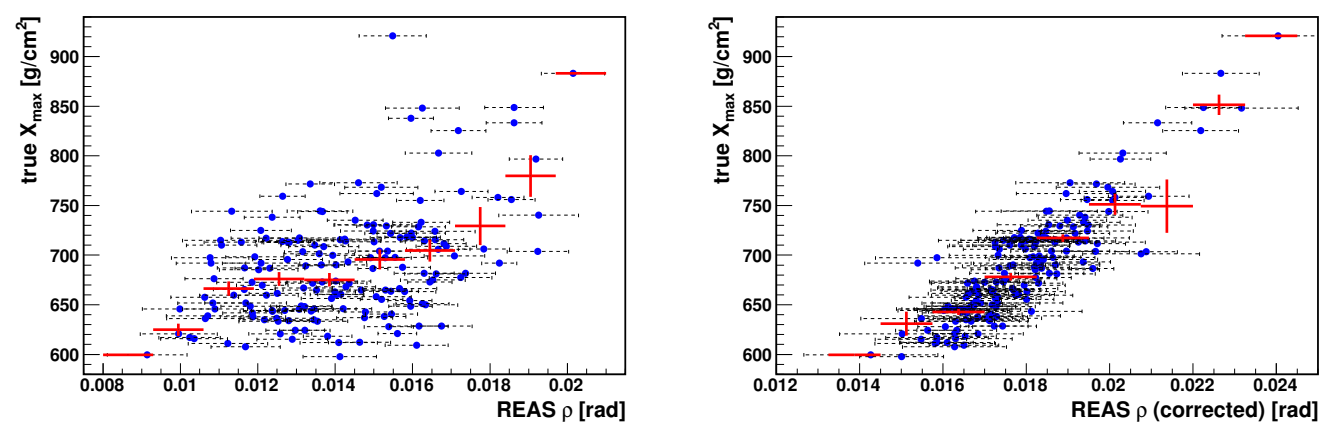

Figure 8.16.: Correlation between the cone parameter $\rho$ and the true $X_{\max }$ of the CORSIKA simulations used as input for REAS3. Left: raw $\rho$ of a conical wavefront fit to the pulse arrival time distributions. Right: after correction for the zenith angle $\theta: \rho$ (corrected $)=\rho \cos ^{-3 / 2} \theta$. Only REAS3 simulations for proton primaries are shown, the simulations for iron primaries behave similarly.
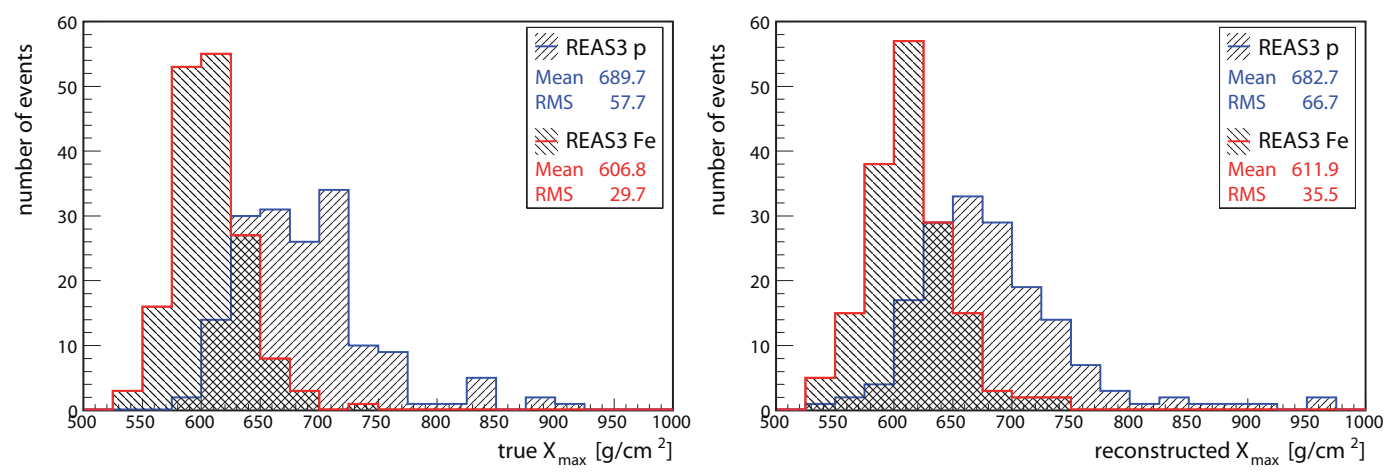

Figure 8.17.: Left: true $X_{\max }$ distribution of the CORSIKA simulations used as input for REAS3. Right: $X_{\max }$ reconstructed with the cone method.
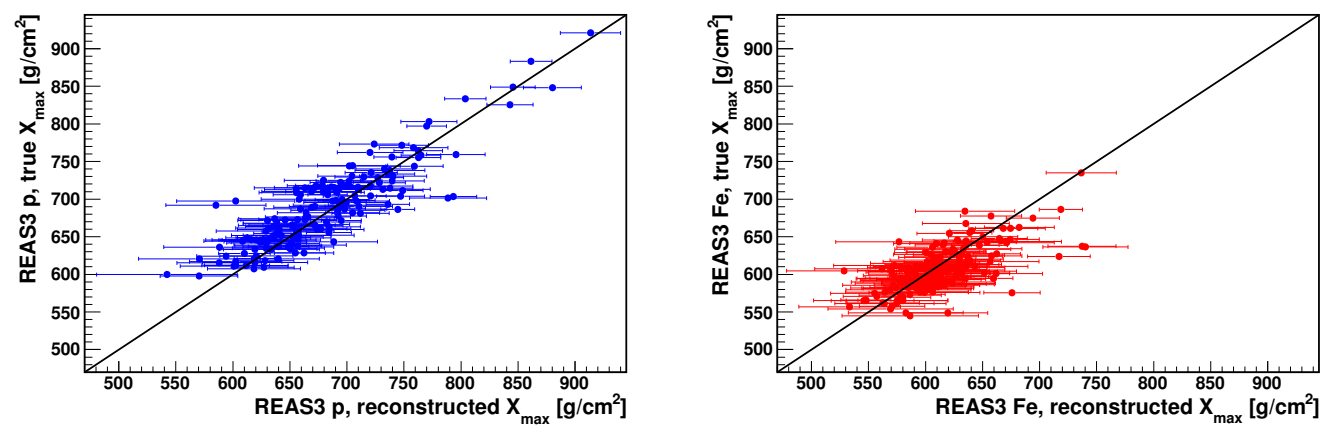

Figure 8.18.: Scatter plots of $X_{\max }$ reconstructed by fitting a conical wavefront versus the true $X_{\max }$ of REAS3 simulations for proton and iron primaries (left and right). 


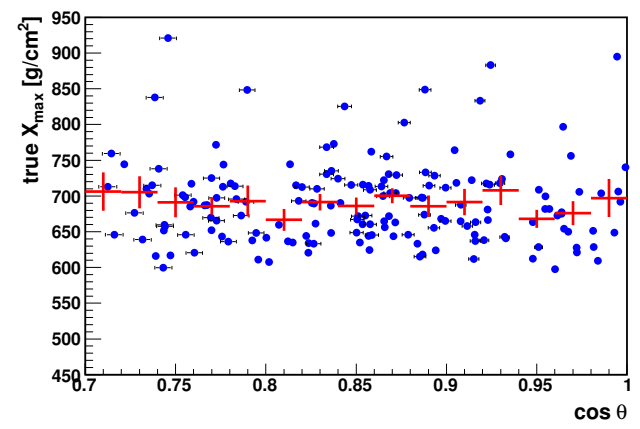

(a) for true $X_{\max }, \mathrm{REAS} 3 \mathrm{p}$

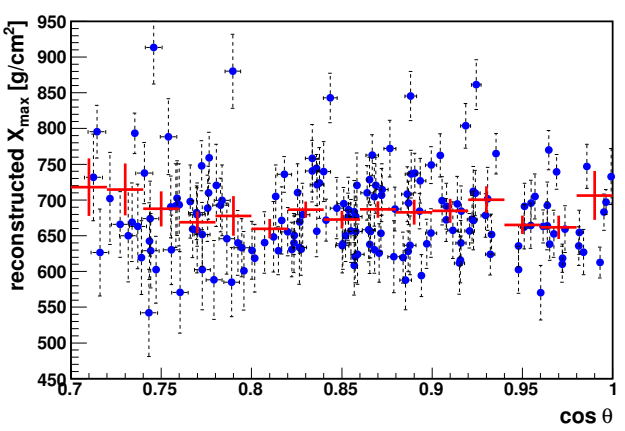

(b) for reconstructed $X_{\max }, \mathrm{REAS} 3 \mathrm{p}$

Figure 8.19.: Neither the true $X_{\max }$ of the CORSIKA simulations used as input for REAS3 nor the $X_{\max }$ reconstructed by fitting a conical wavefront depend on the zenith angle $\theta$. The plots show the simulations for proton primaries. For iron primaries there is no correlation between $X_{\max }$ and $\theta$, either.

$X_{\max }$ is correlated approximately linearly with $\rho_{\text {cor }}$ (see figure 8.16 , right):

$$
X_{\max }=c_{\rho} \cdot \rho_{\text {cor }}
$$

The proportionality constant $c_{\rho}$ is set to $38,000 \mathrm{~g} / \mathrm{cm}^{2} / \mathrm{rad}$ to reproduce the mean $X_{\max }$ of the REAS3 simulations. Indeed, the true $X_{\max }$ of the CORSIKA simulations used as input for REAS3 can be reproduced by this reconstruction method (see figures 8.17 and 8.18). To check whether the reconstructed $X_{\max }$ is really independent of $\theta$, i.e., whether the zenith correction of $\rho$ is reasonable, the true and reconstructed $X_{\max }$ have been plotted against the zenith angle $\theta$ (see figure 8.19), and no correlation between $X_{\max }$ and $\theta$ is observed.

The statistical error of $X_{\max }$ is calculated by propagating the statistical error of $\rho, \Delta \rho$, neglecting all other contributions to the statistical error:

$$
\Delta X_{\max }=c_{\rho} \cdot \Delta \rho_{\mathrm{cor}}=c_{\rho} \cdot \Delta \rho \cos ^{-3 / 2} \theta
$$

The reconstructed $X_{\max }$ for the LOPES events is $783 \pm 216 \mathrm{~g} / \mathrm{cm}^{2}$ (mean and standard deviation), for the REAS3 proton simulations $690 \pm 58 \mathrm{~g} / \mathrm{cm}^{2}$, and for the REAS3 iron simulations $607 \pm 30 \mathrm{~g} / \mathrm{cm}^{2}$ (see figure 8.20). There is a large systematic uncertainty in the absolute scale of $X_{\max }$ which depends on $c_{\rho}$. Changing the time offset in the pulse arrival time calculations for REAS3 by a reasonable amount of $1 \mathrm{~ns}$ will change the absolute scale of $X_{\max }$ by $\sim 100 \mathrm{~g} / \mathrm{cm}^{2}$. Thus, estimating the composition of the primary cosmic rays with LOPES first needs a calibration of $c_{\rho}$. Such a calibration can be either done with an end-to-end simulation including all detector effects, or by a cross-calibration with simultaneous fluorescence measurements, as they will be done at AERA. The cross-calibration with fluorescence measurements is the preferred way to fix $c_{\rho}$, since it is independent of any hadronic interaction models. In any case, it has to be studied, whether $c_{\rho}$ depends on the altitude of 

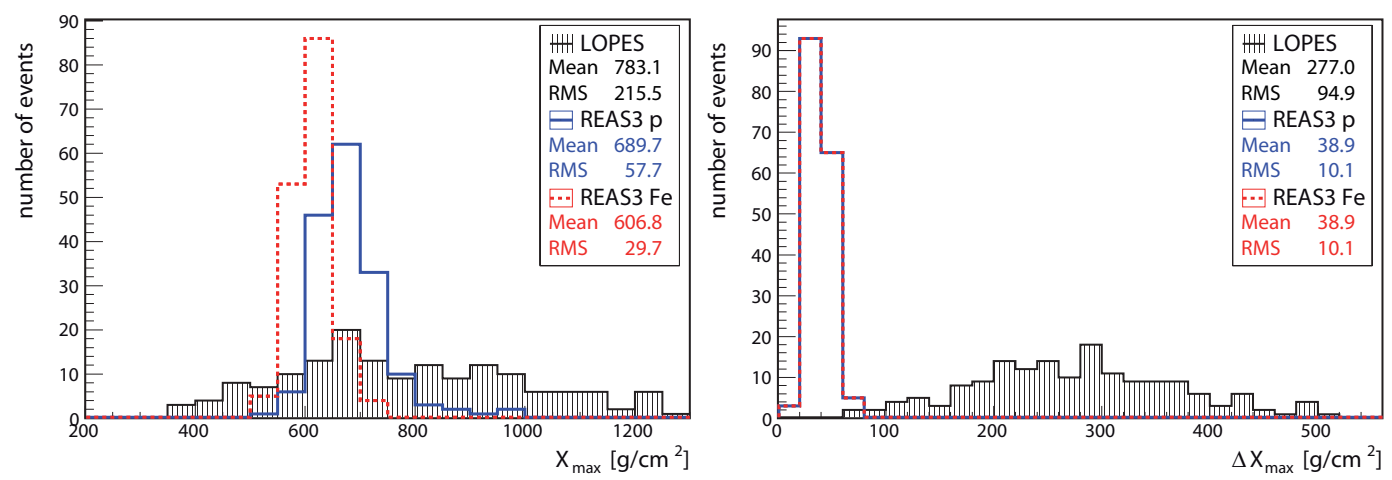

Figure 8.20.: Left: $X_{\max }$ reconstructed by fitting a conical wavefront, for LOPES events and for the corresponding REAS3 simulations for proton and iron primaries. Right: statistical error $\Delta X_{\max }$. The error distributions for REAS proton and iron simulations overlap completely, because $\Delta X_{\max }$ depends only on $\Delta \rho$ which is predominantly determined by the geometry. Thus, for each individual event, $\Delta \rho$ of the proton and the iron simulation is almost equal.

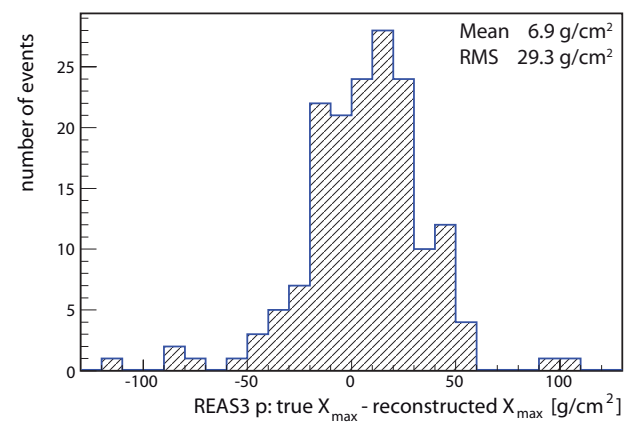

(a) simulations for proton primaries

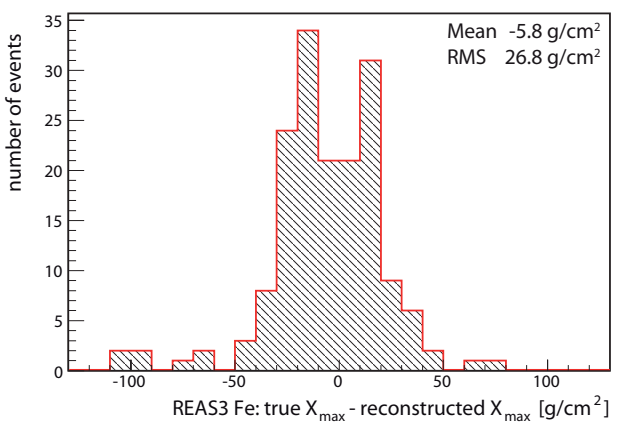

(b) simulations for iron primaries

Figure 8.21.: The $X_{\max }$ resolution is estimated for the REAS3 simulations as the standard deviation (RMS) of the difference between the true $X_{\max }$ and the $X_{\max }$ reconstructed with the cone method.

the experiment $(\sim 1400 \mathrm{~m}$ a.s.l. for AERA, $\sim 110 \mathrm{~m}$ a.s.l. for LOPES $)$ or the local geomagnetic field.

Applying this method, the mean error of the reconstructed $X_{\max }$ is rather large for the LOPES events $\left(\Delta X_{\max } \approx 277 \mathrm{~g} / \mathrm{cm}^{2}\right)$, since the cone parameter $\rho$ typically has a large uncertainty. Also the bias towards a spherical wavefront by the preceding crosscorrelation beamforming might increase the uncertainty of $\rho$. For the simulations, which do not include any uncertainties due to noise, the statistical error of $X_{\max }$ is promisingly small $\left(\Delta X_{\max } \approx 40 \mathrm{~g} / \mathrm{cm}^{2}\right)$. 

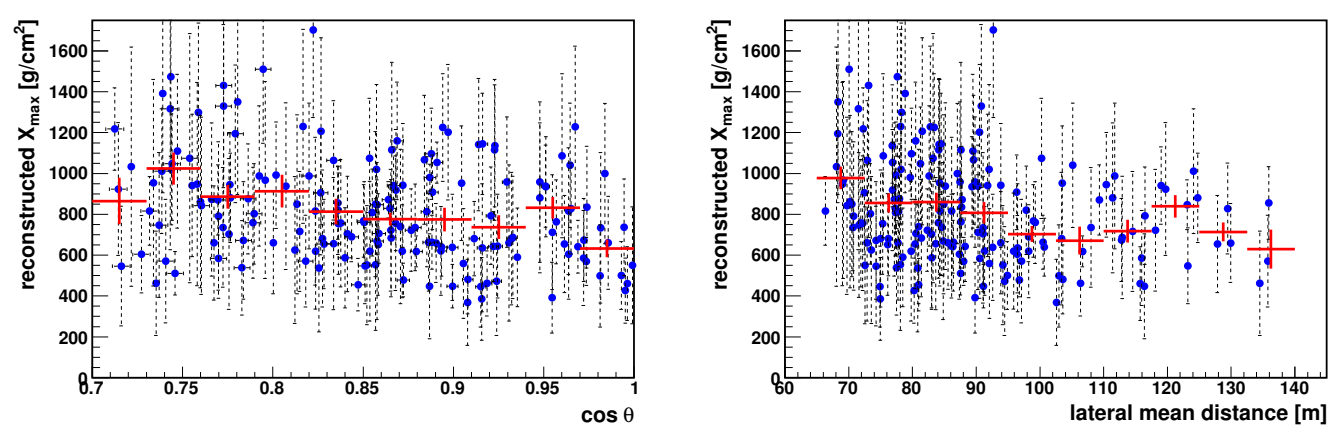

Figure 8.22.: Dependence of the reconstructed $X_{\max }$ of LOPES events on the zenith angle $\theta$ and the lateral mean distance.

The $X_{\max }$ resolution achievable with the cone method is studied with per-event deviations between the true and reconstructed $X_{\max }$ of REAS3 simulations. The spread of the deviations (RMS) is a measure for the resolution. It is about $30 \mathrm{~g} / \mathrm{cm}^{2}$ (see figure 8.21). This is about $25 \%$ smaller than the mean statistical error of the fit, which is a hint that the fitting algorithm of ROOT overestimates the statistical error, and thus the plotted statistical error bars are (slightly) too large.

The accuracy of the cone method for $X_{\max }$ reconstruction can be estimated by the mean deviation between the true and reconstructed $X_{\max }$. The mean deviation is only a few $\mathrm{g} / \mathrm{cm}^{2}$ for proton and iron showers. This is remarkably good keeping in mind the simplicity of the method, which depends only on two shower parameters, $\rho$ and $\theta$, and reconstructs $X_{\max }$ with a simple linear function. Probably, the precision and accuracy can be even improved, if more complex functions are used for the reconstruction of $\rho$ and $X_{\max }$.

Moreover, the cone method for $X_{\max }$ reconstruction has been checked for systematic effects with LOPES events. In particular a possible dependence of $X_{\max }$ on the zenith angle $\theta$ and the lateral mean distance $R_{\text {mean }}$ was checked (figure 8.22), since in both cases a dependence would introduce a systematic uncertainty in the $X_{\max }$ reconstruction. Although the zenith correction of $\rho$ was done according to the dependence found in the simulations, also the reconstructed $X_{\max }$ of the LOPES events is almost independent of $\theta$. There also might be a weak dependence of $X_{\max }$ on $R_{\text {mean }}$ which cannot be clarified due to the large measurement uncertainties. A more conclusive study of the systematic effects will require more precise measurements. Nevertheless, within the measurement uncertainties of LOPES no major systematic effects were found. Consequently, it is reasonable to expect that the cone method works for measured data in principle as well as for the REAS3 simulations, provided that the measurements are precise enough.

Further investigations will be performed at AERA and LOFAR. In particular, it will be checked to which extent the precision of $\rho$ and thus $X_{\max }$ depends on the lateral extension of the experiment, the signal-to-noise ratio and the antenna spacing. Both AERA and LOFAR feature a larger lateral extension than LOPES. AERA has a relatively large antenna spacing, but the advantage of a low ambient noise level. Another advantage of AERA is that the shower reconstruction of the surface array 


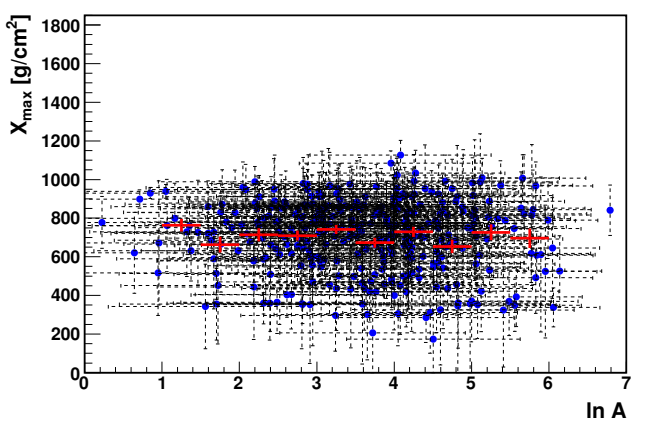

(a) reconstruction with spherical fit

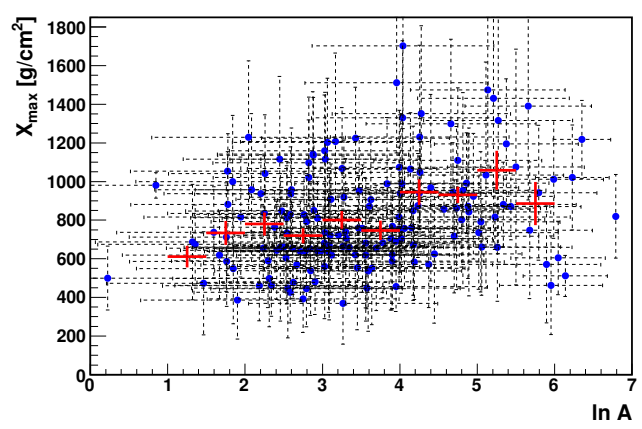

(b) reconstruction with conical fit

Figure 8.23.: $X_{\max }$ is not significantly correlated with a KASCADE-Grande mass estimator based on a combination of the electron and muon numbers. This indicates either that the uncertainties are too large for a per-event mass estimation or that (almost) all primary cosmic rays in the energy range of LOPES are of about the same mass and the scatter in $\ln A$ simply reflects the reconstruction uncertainty of KASCADE-Grande.

can be used as input and cross-check for the analysis of the radio data. LOFAR benefits from a very dense antenna array ideal to reconstruct the radio wavefront in great detail. At both experiments also the application of interferometric methods for the reconstruction of $\rho$ will be tested. This will likely improve the precision of $X_{\max }$, too.

In addition, it will be investigated, whether a precise reconstruction of $\rho$ requires a precise reconstruction of the shower geometry (core and arrival direction). For LOPES, a precise core reconstruction with an uncertainty of only $4-7 \mathrm{~m}$ is provided by KASCADE-Grade, and the arrival direction can be reconstructed by LOPES with an accuracy of $\sim 1^{\circ}[89]$. However, for other radio experiments in particular the core uncertainty could be a problem. In reference [107], it was found that reconstructing $X_{\max }$ with the curvature method requires a precise measurement of the core. On the other hand, this might be different for the cone method. While the core is no distinguished point for a spherical wavefront, a conical wavefront has its apex in the shower core. Thus, it is a promising possibility that fitting the conical wavefront can simultaneously reconstruct $\rho$, the arrival direction and the shower core. Provided that a sufficient number of antennas participates in the measurement, reconstructing the core with the cone method is probably more precise than reconstructing it with the lateral distribution, which seems to flatten towards the shower core.

\subsubsection{Mass sensitivity}

The mass sensitivity of the cone method has already been proven using REAS3 simulations in the previous section. To directly prove the mass sensitivity for LOPES measurements, the reconstructed $X_{\max }$ has to be compared to independently measured mass sensitive shower parameters. Thus, $X_{\max }$ is compared to KASCADEGrande mass estimators based on a combination of the electron and muon numbers 


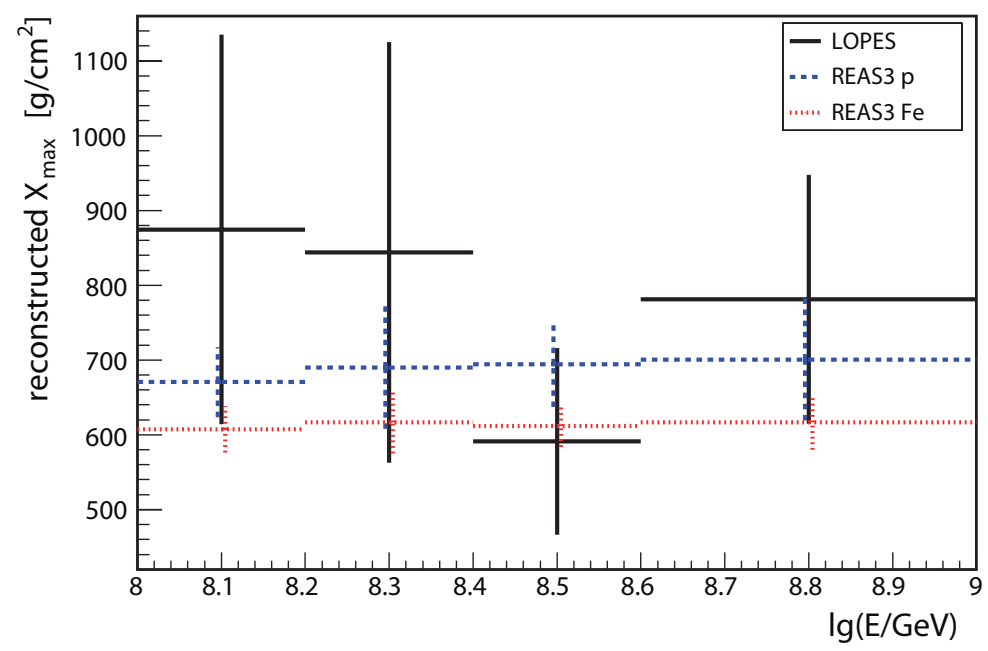

Figure 8.24.: Profiles of the dependence of the reconstructed $X_{\max }$ on the primary energy reconstructed by KASCADE-Grande: for LOPES events and the corresponding REAS3 simulations for proton and iron primaries, respectively. The bars are the spread (RMS) in $X_{\max }$ of the individual events. For the LOPES events, there is also a large systematic uncertainty in the absolute scale of at least $100 \mathrm{~g} / \mathrm{cm}^{2}$.

$[129,141]$ (see figure 8.23). However, due to the large uncertainties of both the reconstructed $X_{\max }$ and the KASCADE-Grande mass estimators, there is no significant correlation, at least for the curvature method (spherical fit of the wavefront). If it all, there is a weak correlation for the cone method (conical fit of the wavefront), but it points into the wrong direction, since $X_{\max }$ is ought to decrease with increasing mass $A$.

Nevertheless, as already shown in figure 8.20, the $X_{\max }$ values reconstructed with LOPES experience a distribution with a peak in the expected order of magnitude. This is a strong hint that the method in principle works also for measurements. The final goal of the method is to study the energy dependent composition of the primary cosmic rays by comparing the $X_{\max }$ of measurements with expectations of simulations, as illustrated in figure 8.24. However, in the case of LOPES, not only the uncertain absolute scale hampers any conclusions on the composition, but also the statistical and systematical errors are simply to large. Nevertheless, the plot gives an impression of the principle potential of the method, but also indicates that a substantial improvement in the measurement precision is required, which is within reach at LOFAR and AERA.

\subsection{Conclusion}

The claim that the wavefront of the radio emission can be better approximated by a conical than by a spherical shape is based on two piece of evidences. First, for LOPES events in general the cone parameter $\rho$ does not depend on the mean lateral distance of the antennas, while the curvature $\kappa$ does. Second, the $\chi^{2}$ of a conical 
wavefront fit to the simulated pulse arrival time distributions is significantly smaller than the $\chi^{2}$ of a spherical wavefront fit. For the LOPES events, the $\chi^{2}$ is about the same for both fits, although the measured LOPES pulse arrival time distributions are biased towards a spherical shape by the preceding cross-correlation beamforming.

Measuring the wavefront shape yields a method for the reconstruction of the atmospheric depth of the shower maximum $X_{\max }$. REAS3 simulations predict that the cone parameter $\rho$ (corrected for the zenith angle) is approximately linearly correlated with $X_{\max }$, which in principle allows an $X_{\max }$ resolution for individual events of at least $30 \mathrm{~g} / \mathrm{cm}^{2}$. The resolution can likely be improved, e.g., by finding an even better approximation to the radio wavefront than a simple cone, or by applying interferometric methods. This implies a direct mass sensitivity of the radio detection method, given that REAS3 describes the radio emission approximately correctly. This is a reasonable assumption since REAS3 is compatible with LOPES data with respect to all tested aspects of lateral distributions (see chapter 7) and pulse arrival time distributions.

However, the mass sensitivity of the radio wavefront could not be definitely proven for LOPES measurements, since the uncertainties of the pulse arrival time measurements are too large. The main reasons for this are the high ambient noise level at LOPES and the small lateral extension of the array. Nevertheless, the distribution of the reconstructed $X_{\max }$ peaks at a value consistent with nuclear cosmic rays. This is a strong hint that the mass sensitivity is also present in the measurements. The $X_{\max }$ distribution can be used to estimate the average $X_{\max }$ in the energy range of $10^{17}-10^{17.5} \mathrm{eV}$, once the absolute scale has been fixed by a cross-calibration with fluorescence or Cherenkov light detectors.

Such a cross-calibration with fluorescence measurements will be done at AERA. With its lower ambient noise level and its larger lateral extension, AERA can probe the shape of the wavefront more precisely, and can compare the results of an interferometric beam-forming pipeline with the results of pulse arrival time measurements in individual antennas. This will reveal, whether interferometry can increase the precision in $X_{\max }$. Moreover, it will be investigated whether the precision in $X_{\max }$ can be improved by combining the reconstruction of the slope of the lateral distribution with the reconstruction of the wavefront. The precision can be tested in a straight forward way by comparing AERA events with fluorescence measurements. Thus, AERA will demonstrate whether radio measurements of cosmic rays are precise enough to compete with traditional detection techniques. 



\section{Conclusion}

Seven years ago, the radio technique was rediscovered for the detection of cosmic ray air showers. This thesis aims to answer some of the remaining questions on the achievable performance of this technique. LOPES confirmed that in principle all important properties of the primary cosmic ray particles (energy, arrival direction, and mass) can be reconstructed, but it is still open, whether the precision of digital radio arrays can compete with other techniques. This thesis reflects the progress in the understanding of the radio emission and presents a set of methods which improved LOPES and will help to answer the open questions with AERA and LOFAR, the next generation digital radio arrays.

LOPES made great strides towards establishing digital radio interferometry for air shower detection. Interferometric methods like cross-correlation beamforming increase the signal-to-noise ratio, hence lower the detection threshold and improve the efficiency and reconstruction accuracy. With a lateral extension more than 10 times larger than LOPES, AERA has the means to test the limitations of radio interferometry for air shower detection. The results will help in the decision how to design future radio experiments, e.g. whether it is worth equipping larger arrays for the highest energies with expensive electronics suitable for interferometry - a valuable but costly tool - or if cheaper electronics without this ability might be an option.

Digital radio interferometry requires a measurement of the time-dependent electric field strength (measuring only the pulse power is not sufficient). Furthermore, a relative timing precision and accuracy of $\sim 1 \mathrm{~ns}$ is necessary, which can be achieved by the combination of several methods. These include sophisticated analog and digital electronics with known phase properties, e.g., to correct for pulse distortion in a subsequent analysis. However, experience with LOPES and the AERA prototype setups revealed that this alone is insufficient. In addition, a reference beacon is required to continuously monitor and improve the relative timing. The feasibility of the beacon technique was demonstrated in this thesis. It is applied at LOPES and AERA, enabling their use as digital radio interferometers.

Close to the detection threshold of digital radio antenna arrays at $\sim 10^{17} \mathrm{eV}$, and for LOPES in general, measurements are limited by radio background of natural and human origin. Due to the short wave and FM radio bands only band-limited measurements are practicable, but in a band-limited measurement, any RFI pulse will look nearly identical to air shower induced pulses. For this reason, LOPES would have no chance to identify most of its events, if not operated in coincidence with KASCADE-Grande. Only with the initial input of the KASCADE-Grande reconstruction, the radio pulse can be identified with interferometric methods. A radio stand-alone detection might be feasible at best for events with $E>10^{18} \mathrm{eV}$, when air shower pulses can be distinguished from other radio sources in the sky by their lateral characteristics, i.e., the lateral distribution of amplitudes and arrival times. 
Thus, in any radio measurements of air showers, and in particular at LOPES, special care has to be paid to the background consisting of noise and RFI. While RFI can partially be suppressed by digital data conditioning, noise has to be treated in a consistent way with the signal measurements. The influence of noise is different from usual particle physics experiments, since radio noise can interfere destructively and constructively. Established methods known from communication engineering are not fully sufficient, either. This thesis presents a method how to treat the influence of noise on pulse amplitude and arrival time measurements in individual antennas.

The recent progress in understanding the radio emission is manifested in two results presented in this thesis: the improved description of measurements by simulations, and the better understanding of the radio wavefront. It is shown that the improved radio simulation software REAS3 can in general reproduce lateral distributions measured with LOPES. Furthermore, REAS3 predicts that the radio wavefront is approximately conical which is also in agreement with LOPES measurements. For the first time, a radio simulation thus describes measured data approximately correctly. In particular, it is remarkable that REAS3 also predicts the absolute amplitude correctly, although REAS3 has no free parameter. However, radio simulations do not yet reach the same level of predictivity as air shower simulations for particle surface, fluorescence, and Cherenkov detectors reach. Besides overcoming the technical limitations of present experiments, e.g., the small extensions of LOPES and other currently operating radio antenna arrays, an adequate theoretical comprehension is the main challenge of the next few years in the field of cosmic ray radio detection.

It had long been predicted that radio arrays should be sensitive to $X_{\max }$, the atmospheric depth of the shower maximum, and thus to the type and mass of the primary particle. This thesis gives experimental indication supporting this prediction. Given that REAS3 describes the radio emission approximately correct, it could be shown successfully that there are, in principle, two methods for mass estimation: a reconstruction of the radio wavefront with pulse arrival time measurements and a per-event comparison of lateral distributions with simulations, since the opening angle of the conical wavefront and the slope of the lateral distribution are sensitive to $X_{\max }$. The limited lateral extension of LOPES and the high ambient noise level, however, cause large uncertainties and inhibit per-event estimations of $X_{\max }$.

Giving an outlook, the prospects of digital radio arrays lie in hybrid experiments. Future cosmic ray observatories could combine particle surface, fluorescence, Cherenkov and digital radio detectors. The surface detector can determine the exposure, and reject radio events triggered by RFI pulses. The additional radio, fluorescence or Cherenkov measurements can improve the reconstruction accuracy of the primary energy and mass, like it is currently done at the Pierre-Auger-Observatory and the Telescope Array. It is sufficient to equip only a relatively small area of future experiments with fluorescence or Cherenkov telescopes to gain enough statistics at $E<10^{19} \mathrm{eV}$. For higher energies, the full area could be equipped with radio antennas providing high quality energy and mass measurements at $E>10^{18} \mathrm{eV}$ with a duty cycle close to $100 \%$. This would increase the rate of high quality events at the highest energies by a factor of $\sim 10$ compared to the established combination of surface and fluorescence detectors, without requiring a larger detection area. 


\section{A. Radio Measurements at Tunka}

The Tunka experiment measures Cherenkov light emitted by air showers [59]. It is located close to Irkutsk, Siberia, Russia, and consists of 133 photomultiplier detectors (with $20 \mathrm{~cm}$ photo cathode diameter) in a hexagonal structure (see figure A.1). Since Cherenkov measurements are sensitive to the longitudinal shower development, they allow a relatively good estimation of the primary energy and mass. Tunka operates in about the same energy range as $\operatorname{KASCADE}-$ Grande $\left(E \lesssim 10^{18} \mathrm{eV}\right)$ and will yield a complementary measurement of the cosmic ray energy spectrum and its composition.

Since Tunka is located in a radio-quiet rural area, it is an interesting site for simultaneous operation of a digital radio array. The additional radio measurements of air showers will have two purposes. First, the radio measurements can be crosscalibrated with the Cherenkov measurements with respect to the energy and $X_{\max }$. This way, Tunka can improve the understanding of the radio emission mechanisms, which in turn is a valuable input for other radio experiments. Second, Tunka can profit from the radio measurements, e.g., to improve the angular resolution with a hybrid reconstruction. This will be similar to the situation at the Pierre Auger Observatory, where the reconstruction of air showers measured with fluorescence telescopes is significantly enhanced by using the information of a simultaneous surface detector measurement. To increase the event statistics of Tunka at high energies, it might also be possible to continue radio measurements at day or during bad weather, when the PMTs cannot operate. However, for a radio stand-alone operation, first a reliable self-trigger has to be developed, like it is presently done for AERA.

To pave the way for radio measurements at Tunka, a SALLA antenna was installed in summer 2009, using AERA prototype hardware developed within the LOPES ${ }^{\text {STAR }}$ setup. This antenna is located in one of the inner Tunka clusters, cluster 7 , close to the central PMT detector and the DAQ electronics box of this cluster. The close distance to the electronics was chosen to facilitate the installation of the SALLA. However, it can be disadvantageous if the electronics emit RFI.

The results of the test measurements with this prototype antenna are presented in this appendix. Several radio candidate events have been found within half a year of data taking. There is evidence that the radio pulses in the candidate events are linked to cosmic ray air showers. However, it could not finally be resolved whether the detected radio pulses are directly emitted by the air shower, or whether they are caused by crosstalk or secondary RFI pulses emitted by the Tunka PMTs or the DAQ electronics. The following paragraphs summarize the analysis. A more detailed description is available in reference [142], and a short summary of the results is published in reference [143].

In September 2009, a SALLA with two polarization channels, east-west and northsouth, were deployed at Tunka. The AERA prototype electronics filters the signal to a limited bandwidth of $30-80 \mathrm{MHz}$. The amplified and filtered radio signal is digitized with a sampling frequency of $200 \mathrm{MHz}$ by the same Tunka electronics 

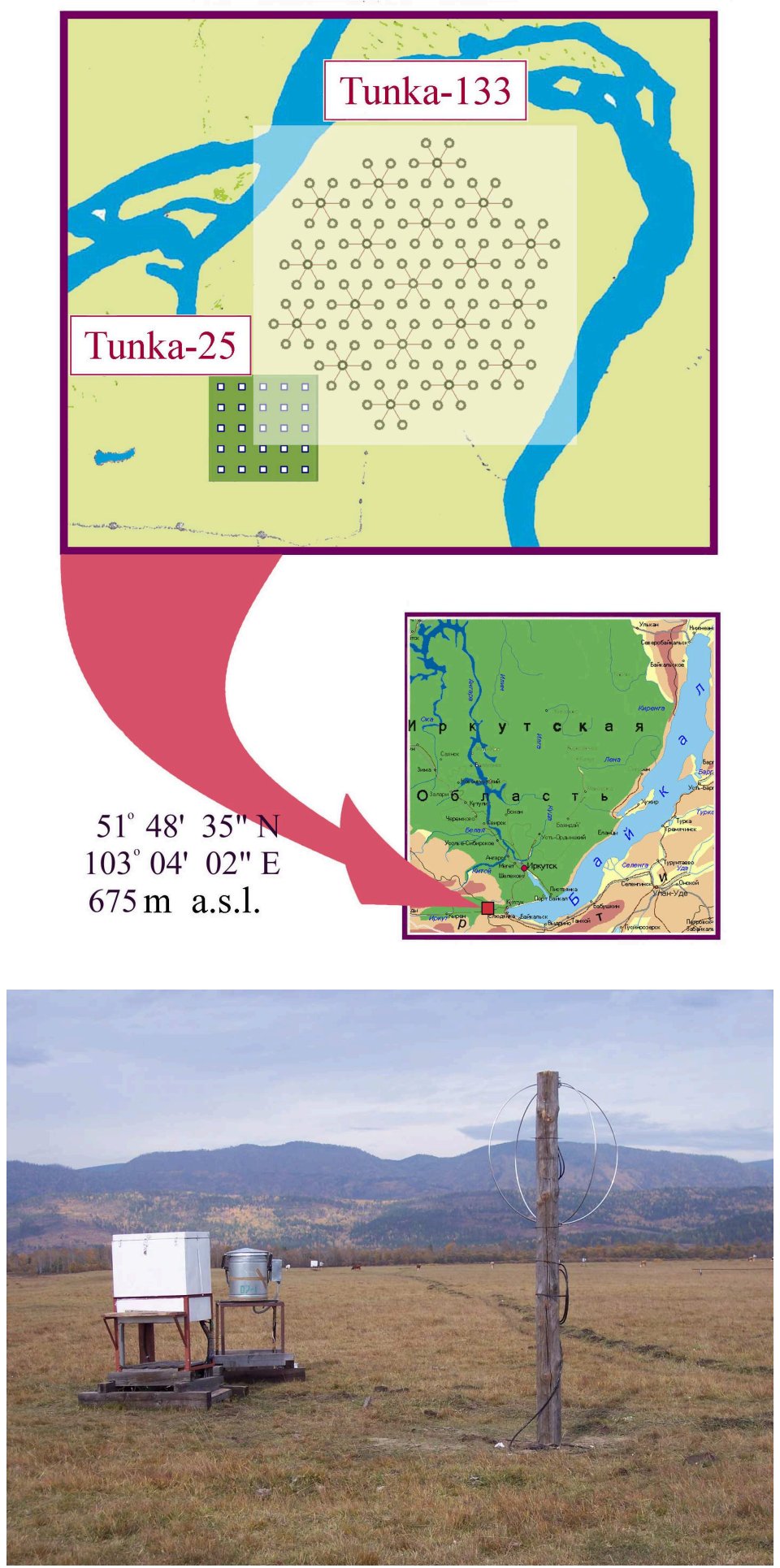

Figure A.1.: Top: Map of the Tunka experiment with a hexagonal structure of photomultiplier detectors for Cherenkov light emitted by air showers [144]. It covers an area of $\sim 1 \mathrm{~km}^{2}$.

Bottom: SALLA installed at Tunka cluster 7, cluster DAQ electronics box (white box), and central photomultiplier detector. 


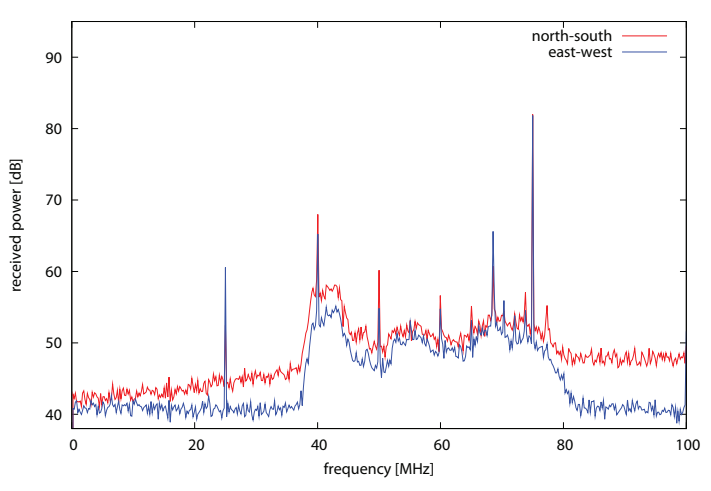

(a) background spectrum

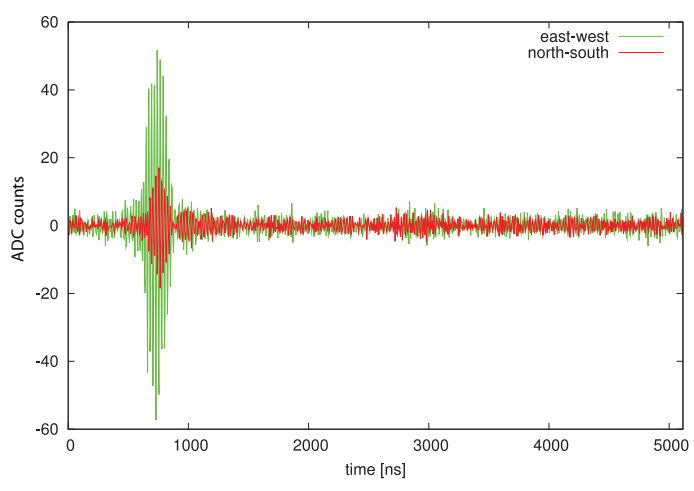

(b) example radio event

Figure A.2.: Background spectrum measured with the SALLA at Tunka cluster 7, averaged over 100 successive events, and an example for a radio pulse detected with a threshold algorithm in both polarization channels of the SALLA . Most likely it is a RFI pulse because it is not in coincidence with PMT pulses typically occurring at $2-3 \mu \mathrm{s}$.

used to digitize the PMT signals. The same trigger is used for the PMTs and the SALLA. Whenever cluster 7 is triggered, both channels of the SALLA are read out simultaneously with the seven PMT detectors of this cluster. The trace length is $5.12 \mu \mathrm{s}$.

Analyzing the first radio data includes several steps: First, a general investigation of the radio background at Tunka. Second, a check if the SALLA works reliably. Third, a search for any radio pulses. Forth, an investigation whether there are radio pulses causally linked to air showers by correlating with the Tunka reconstruction of air shower events. And fifth, a careful analysis, if radio candidate events could also be caused by crosstalk or RFI.

About 200,000 events from 6 months have been analyzed, limited to the Tunka measurement periods during dark moonless nights. To check for the system health and stability of the SALLA, background spectra have been evaluated for each month (see figure A.2 for an example). The background spectrum did not change significantly over time, even in the cold winters of Siberia $\left(T_{\min } \approx-40^{\circ} \mathrm{C}\right)$, which demonstrates the stability of the system. The spectrum shows several peaks of narrow band RFI within the measurement bandwidth of $30-80 \mathrm{MHz}$. These peaks can be mitigated during analysis, like it is usually done in LOPES and AERA data conditioning. In general, the background level is much lower than at LOPES, and might be comparable with the situation at AERA in Argentina. However, a final evaluation of the background will require absolute calibrated measurements at Tunka.

Radio pulses have been found in about $8 \%$ of the events with a threshold algorithm (see figure A.3 (left)). Since the arrival time of these radio pulses is mostly random, they probably originate from uncorrelated background perturbations. However, the situation changes if an additional threshold cut is applied on the PMT signal. When high energy events are selected, a fraction of the radio pulses is detected at about 


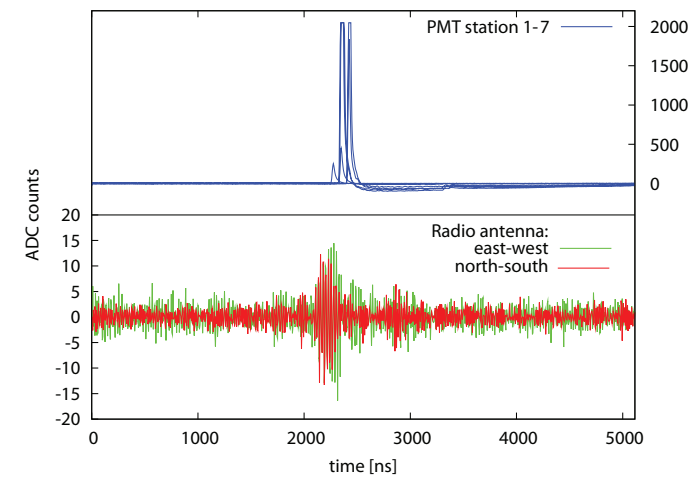

(a) example for coincident radio pulse

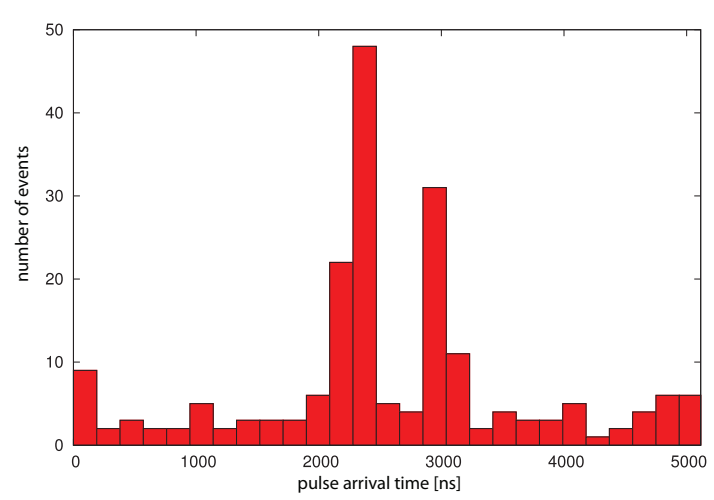

(b) arrival time distribution

Figure A.3.: Left: Example event with a radio pulse coincident with PMT pulses. Right: Arrival time distribution of radio pulses in coincidence with Tunka events, in which at least 5 of the 7 PMTs of cluster 7 exceeded a threshold of 70 ADC counts, i.e., a SNR of $\approx 20$. The first peak is only visible for high energy events and roughly at the same time as the PMT pulses. Hence, radio pulses at this time are candidate events. The second peak is already visible without any threshold cut on the PMTs, and probable arises from RFI or crosstalk.

the same time than the PMT pulses (see figure A.3 (right)). This way, 74 events are selected, in which the radio pulse is coincident with strong PMT pulses. For only 50 of these 74 events also a Tunka reconstruction is available, since the remaining 24 events have been recorded either during test runs or bad weather. Thus, there are 50 radio candidate events.

Apart from the coincident arrival time with the PMT pulses, there is additional evidence that the radio candidate events are linked to air showers. A selection of 78 Tunka events with energy $E>10^{17} \mathrm{eV}$, zenith angle $\theta<45^{\circ}$, and lateral distance to the SALLA $R \lesssim 300 \mathrm{~m}$ contains 10 of the 50 candidate events - much more than expected by chance. Furthermore, there is a clear correlation of these 10 radio candidates with the energy and the lateral distance: there is an accumulation of events close to the SALLA and at high primary energy. This is expected if the radio pulses are by any means caused by air showers. However, it is not enough to proof that the detected pulses originate directly form the air shower, since the same correlation is expected for Cherenkov pulses detected by the PMTs. Consequently, any secondary RFI pulses caused by the PMT or crosstalk in the electronics would exhibit the same correlation between the radio pulses and the energy and lateral distance. A definite proof that the radio pulses are directly emitted by the air shower, requires the investigation of a shower parameter which is correlated with radio and Cherenkov pulses in a distinct way, e.g., the geomagnetic angle. This is currently analyzed [142].

Summarizing, the analysis of the first Tunka radio data showed the difficulty to judge with only one antenna if detected radio pulses originate directly from air show- 


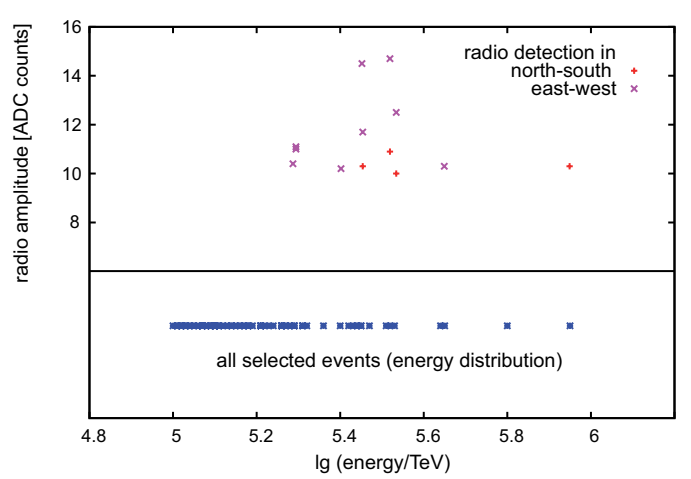

(a) energy $E$

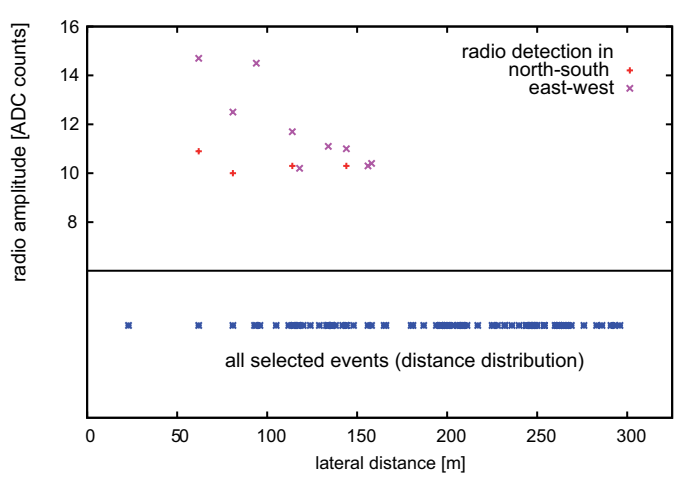

(b) lateral distance $R$

Figure A.4.: Energies $E$ and lateral distances $R$ of Tunka events selected with $E>10^{17} \mathrm{eV}, \theta<45^{\circ}$ and $R<300 \mathrm{~m}$, and amplitude of the radio candidate events contained in the selection. The correlation matches the expectations for radio pulses originating from air showers: The radio amplitude should be roughly proportional to the primary energy, and should be higher at small lateral distances. Thus, the detection probability should increase with $E$, and decrease with $R$.

ers or not. At least a fraction of the 50 radio candidate events might be caused either by RFI emitted by the PMTs or crosstalk inside of the electronics box. In the first case, it is advisable to place further antennas as far away from the PMTs as possible, i.e., in the center between three PMTs. Then, primary air shower pulses and secondary PMT pulses can be distinguished by their arrival times. In the latter case, the shielding inside of the electronics box has to be improved. Taking data over a longer period with a disconnected antenna will allow to distinguish between both cases. No external radio pulses, but only RFI pulses cause by crosstalk should still be present in the data if the antenna is disconnected.

Furthermore, a comparison of detected radio pulses with REAS3 simulations can help to identify radio pulses emitted by air showers. Since no absolute calibration is available for the SALLA at Tunka, yet, the absolute amplitude of the radio pulse is not a good quantity for this comparison. Nevertheless, the amplitude ratio between both polarization channels will be a good quantity, since it is independent of the absolute calibration. Once 'real' pulses have been identified, the comparison with REAS3 can even yield a rough absolute calibration, because REAS on average predicts the correct amplitude, as proven by comparisons with the absolute calibrated LOPES experiment (see chapter 7).

Finally, more antennas will be needed. With three or more antennas, a reconstruction of the arrival direction, and an estimation of the primary energy and mass will be possible. A comparison of these parameters to the Tunka reconstruction will help in the decision, whether an additional radio array can improve the reconstruction of high energy events sufficiently to make the effort worthwhile. A beacon can be used to improve the relative timing precision from $\sim 10 \mathrm{~ns}$ to $\sim 1 \mathrm{~ns}$ required for digital radio interferometry, which will lower the detection threshold and increase the reconstruction quality. 



\section{B. Up-Sampling and Envelope}

The LOPES standard analysis pipeline (section 3.2.2) includes two major steps of signal conditioning: up-sampling and calculation of an envelope by a Hilbert transform (Hilbert envelope). These two steps increase the precision of the amplitude and arrival time measurement of a radio pulse. Theoretically, in ideal circumstances, the precision could be arbitrarily high - only limited by computing time. Practically, the precision of amplitude and arrival time measurements is still limited by calibration uncertainties and noise (chapter 6), but, after up-sampling, not anymore by the sampling rate.

The value of any measured physical function, like the electric field strength, cannot change arbitrarily fast with time. There exists a maximum frequency with which the value can change, and sometimes also a minimum frequency. For example at LOPES, a bandpass filter ensures that the electrical field strength is measured in the frequency band from $40-80 \mathrm{MHz}$ only. Hence, the measured field strength (signal and noise) must be a sum of oscillations with frequencies between 40 and $80 \mathrm{MHz}$. Any oscillations with other frequencies would be unphysical. This is called a band-limited measurement.

Consequently, it is not necessary to sample the electric field strength at an infinite rate to record the full physical information. According to the Nyquist theorem (sampling theorem) [87], it is sufficient to sample a band-limited function with a frequency of twice the bandwidth. Then, any values between the samples are welldefined, and can be retrieved by up-sampling which is the correct way of interpolation. Up-sampling increases the number of samples without adding any further physical information. In comparison to other methods of signal conditioning, for instance fitting a wave form or applying a matched filter, up-sampling requires no further presumptions on the band-limited signal.

There exist several up-sampling algorithms (see e.g., [145]). The one used in the softwares of LOPES and AERA is zero-padding in the frequency domain, which consists of several steps and involves Fast Fourier Transforms (FFTs).

1. FFT on the time-series of the electric field strength:

By a FFT of the time-series, the frequency spectrum is obtained. In the case of LOPES, frequencies are in descending order since the electric field strength has been sampled in the second Nyquist domain (i.e., from $80-40 \mathrm{MHz}$ ). Hence, as first step after the FFT, the order has to be reverted to ascending frequencies. In the case of AERA, data are sampled in the first Nyquist domain, and frequencies are already in ascending order. ${ }^{1}$

\footnotetext{
${ }^{1}$ In principle, obtaining a physical meaningful normalization of the first and the last data point of the FFT requires some effort. However, by design of the analog bandpass filter, the influence of these boundary bins is strongly suppressed and the correct normalization becomes unimportant.
} 


\section{Zero Padding:}

The spectrum is extended and filled with zeros. Therefore, additional frequencies are inserted up to a frequency $N \in \mathbb{N}$ times the original band-width, keeping the frequency spacing of the FFT. In the case of LOPES, this means that the additional frequencies are inserted from $0-40 \mathrm{MHz}$ and above $80 \mathrm{MHz}$. This way, the number of data points in the frequency spectrum is effectively increased by the up-sampling factor $N$. The inserted data points reflect that the power of the signal is zero outside of the measurement bandwidth.

3. FFT of the frequency spectrum:

The zero-padded frequency spectrum is transformed back to the time domain. The resulting time-series of the electrical field strength contains $N$ times more data points than the original time-series. All original data points have been conserved and the new data points are the physical correct interpolation of the band-limited time-series.

By making use of up-sampling, the coarse sampling rate of LOPES does not anymore limit the measurement precision. This has successfully been tested at LOPES, using test pulses emitted at different times with respect to the sampling clock of $12.5 \mathrm{~ns}$. Hence, the measurement precision of LOPES is only limited by the calibration and noise.

The Hilbert envelope is the instantaneous amplitude of the electrical field strength [145]. The Hilbert envelope is always positive. Its maximum is usually slightly higher than the maximum of the absolute electrical field strength (a few $\%$ at LOPES), and at a slightly different time (a few ns at LOPES). In the frame of LOPES ${ }^{\text {STAR }}$, several methods for envelope calculation have been investigated, deeming the Hilbert envelope as preferred method [82]. Thus, the Hilbert envelope is used in this thesis to determine pulse arrival times and amplitudes.

Calculating the Hilbert envelope is based on the Hilbert transform $F_{H i}(t)$ which in communication engineering is also known as quadrature function of $f(t)$, and is defined as:

$$
F_{H i}(t)=\frac{1}{\pi} \int_{-\infty}^{+\infty} \frac{f\left(t^{\prime}\right)}{t^{\prime}-t} \mathrm{~d} t^{\prime}
$$

The Hilbert envelope is defined as $\left|f(t)-i F_{H i}(t)\right|$, when $f(t)$ is the (up-sampled) time-series of the electrical field strength. Throughout this thesis, there are several examples for Hilbert envelopes (e.g., figures 4.7 and 6.3). For further reading, any text books about communication engineering, signal processing and Fourier transforms are recommended, for instance [145].

Furthermore, setting the boundary bins plainly to zero is an elegant way to remove any unphysical DC offset from the measurement. 


\section{LOPES Antenna Positions}

This appendix lists the positions and polarizations (alignments) of the LOPES 30 and LOPES Dual setups. For the definition of the coordinate system and a discussion of the measurement uncertainties see section 4.3.

Table C.1.: Antenna positions of the LOPES 30 setup, height relative to antenna 1.

\begin{tabular}{ccrccc}
\hline Antenna & ID & Northing $x[\mathrm{~m}]$ & Easting $y[\mathrm{~m}]$ & Height $z_{1}[\mathrm{~m}]$ & Polarization \\
\hline 1 & 10101 & 79.61 & -45.96 & 0 & EW \\
2 & 10102 & 111.43 & -64.30 & -0.46 & EW \\
3 & 10201 & 36.12 & -84.72 & -0.35 & EW \\
4 & 10202 & 54.62 & -52.92 & -0.06 & EW \\
5 & 20101 & 22.68 & -34.59 & -0.08 & EW \\
6 & 20102 & 40.84 & -2.65 & 0.07 & EW \\
7 & 20201 & 2.11 & 40.76 & 0.01 & EW \\
8 & 20202 & 34.35 & 22.47 & 0.06 & EW \\
9 & 30101 & 72.80 & -21.04 & -0.09 & EW \\
10 & 30102 & 91.02 & 10.97 & -0.19 & EW \\
\hline 11 & 40101 & -64.34 & -112.27 & -0.27 & EW \\
12 & 40102 & -45.98 & -80.04 & -0.24 & EW \\
13 & 40201 & -84.67 & -36.80 & -0.19 & EW \\
14 & 40202 & -52.07 & -54.61 & -0.20 & EW \\
15 & 50101 & -91.56 & -11.62 & -0.15 & EW \\
16 & 50102 & -73.14 & 20.12 & -0.08 & EW \\
17 & 50201 & -112.22 & 63.41 & 0.17 & EW \\
18 & 50202 & -80.26 & 45.14 & 0.06 & EW \\
19 & 60101 & -34.72 & -23.30 & -0.14 & EW \\
20 & 60102 & -2.71 & -41.54 & -0.24 & EW \\
\hline 21 & 70101 & -13.86 & -98.16 & 0.01 & EW \\
22 & 70102 & 4.34 & -66.26 & 0.00 & EW \\
23 & 70201 & -41.35 & 1.88 & -0.19 & EW \\
24 & 70202 & -23.05 & 33.92 & -0.30 & EW \\
25 & 80101 & -61.69 & 77.36 & -0.11 & EW \\
26 & 80102 & -29.84 & 58.84 & -0.27 & EW \\
27 & 80201 & -21.85 & -152.55 & -0.35 & EW \\
28 & 80202 & -7.83 & -127.94 & -0.18 & EW \\
29 & 90101 & 16.85 & -141.97 & -0.01 & EW \\
30 & 90102 & 2.63 & -166.64 & -0.40 & EW \\
\hline & & & & & \\
\hline
\end{tabular}


Table C.2.: Antenna positions of the LOPES Dual setup, height relative to the ground plane of KASCADE.

\begin{tabular}{ccrccc}
\hline Antenna & ID & Northing $x[\mathrm{~m}]$ & Easting $y[\mathrm{~m}]$ & Height $z_{K}[\mathrm{~m}]$ & Polarization \\
\hline 1 & 10101 & 79.73 & -45.60 & 1.12 & NS \\
2 & 10102 & 111.59 & -63.98 & 1.05 & EW \\
3 & 10201 & 36.31 & -84.52 & 1.19 & NS \\
4 & 10202 & 54.80 & -52.61 & 1.13 & EW \\
5 & 20101 & 22.72 & -34.17 & 1.07 & EW \\
6 & 20102 & 22.72 & -34.17 & 1.07 & NS \\
7 & 20201 & 2.18 & 41.15 & 1.21 & NS \\
8 & 20202 & 34.48 & 22.88 & 1.16 & EW \\
9 & 30101 & 72.72 & -20.68 & 1.06 & EW \\
10 & 30102 & 91.02 & 11.28 & 0.96 & NS \\
\hline 11 & 40101 & -64.10 & -111.95 & 1.27 & NS \\
12 & 40102 & -45.77 & -79.76 & 1.30 & EW \\
13 & 40201 & -84.46 & -36.48 & 1.35 & EW \\
14 & 40202 & -52.31 & -54.49 & 1.25 & NS \\
15 & 50101 & -91.37 & -11.36 & 1.38 & NS \\
16 & 50102 & -72.91 & 20.41 & 1.47 & EW \\
17 & 50201 & -111.81 & 63.48 & 1.53 & EW \\
18 & 50202 & -79.92 & 45.56 & 1.53 & NS \\
19 & 60101 & -34.37 & -22.83 & 1.28 & EW \\
20 & 60102 & -34.37 & -22.83 & 1.28 & NS \\
\hline 21 & 70101 & -13.70 & -97.84 & 1.53 & EW \\
22 & 70102 & 4.51 & -65.93 & 1.50 & EW \\
23 & 70201 & -41.18 & 2.16 & 1.32 & NS \\
24 & 70202 & -22.79 & 34.33 & 1.21 & EW \\
25 & 80101 & -61.46 & 77.65 & 1.43 & EW \\
26 & 80102 & -29.63 & 59.18 & 1.28 & NS \\
27 & 80201 & -7.55 & -127.63 & 1.39 & NS \\
28 & 80202 & -7.55 & -127.63 & 1.39 & EW \\
29 & 90101 & -13.70 & -97.84 & 1.53 & NS \\
30 & 90102 & 4.51 & -65.93 & 1.50 & NS \\
\hline & & & & &
\end{tabular}




\section{Example Events}

This appendix tries to give an unbiased overview over LOPES events. Due to the large number of LOPES events, only a small subset of events can be shown. Six events for each of the four selections (see table 7.2) have been chosen by an automated procedure. These four selections are: events from the LOPES 30 and LOPES Dual setups with core inside of the KASCADE and Grande array, respectively. Only events passing all selection and quality cuts are shown (cf., tables 7.3 and 8.1).

For each event, there are three pairs of plots:

\section{Traces and cross-correlation beam}

Left: Calibrated electrical field strength in individual antennas (different colors), upsampled to $1280 \mathrm{MHz}$, after beamforming, i.e., traces are shifted by the geometrical delays.

Right: Smoothed cross-correlation beam (dark blue), Gaussian fit to the smoothed cross-correlation beam (light blue), power beam (brown).

\section{Lateral distribution}

Lateral distribution of pulse amplitudes in individual antennas, and an exponential fit with an amplitude parameter $\epsilon_{100}$ and a slope parameter $R_{0}$. In addition to the LOPES measurements, the corresponding REAS3 simulation selected by the muon number are shown (left: for proton primaries; right: for iron primaries). For the simulated data points there are no error bars.

\section{Pulse arrival time distribution}

Distribution of pulse arrival times in individual antennas, a spherical wavefront fit with a curvature radius $r_{c}$ (left), and a conical wavefront fit with a cone parameter $\rho$ (right). The corresponding REAS3 simulation selected by the muon number are shown for proton primaries (blue full squares) and for iron primaries (red open squares). For the simulated data points there are no error bars.

The arrival direction shown in the plots is reconstructed with LOPES by crosscorrelation beamforming, but the shower parameters given in the figure caption are reconstructed by KASCADE or Grande, respectively. They are the zenith angle $\theta$, the azimuth angle $\phi$, the geomagnetic angle $\alpha$, the energy $E$, and the mass of the primary particle $A$ given as $\ln A$ ( $\ln A=0$ corresponds to a proton, and $\ln A \approx 4$ to an iron nucleus). While all angles are measured very precisely with KASCADEGrande (better than $1^{\circ}$ ), the energy $E$ and the mass $A$ are only estimated roughly with typical errors of $20-40 \%$ for the energy and about \pm 2 for $\ln A$. 

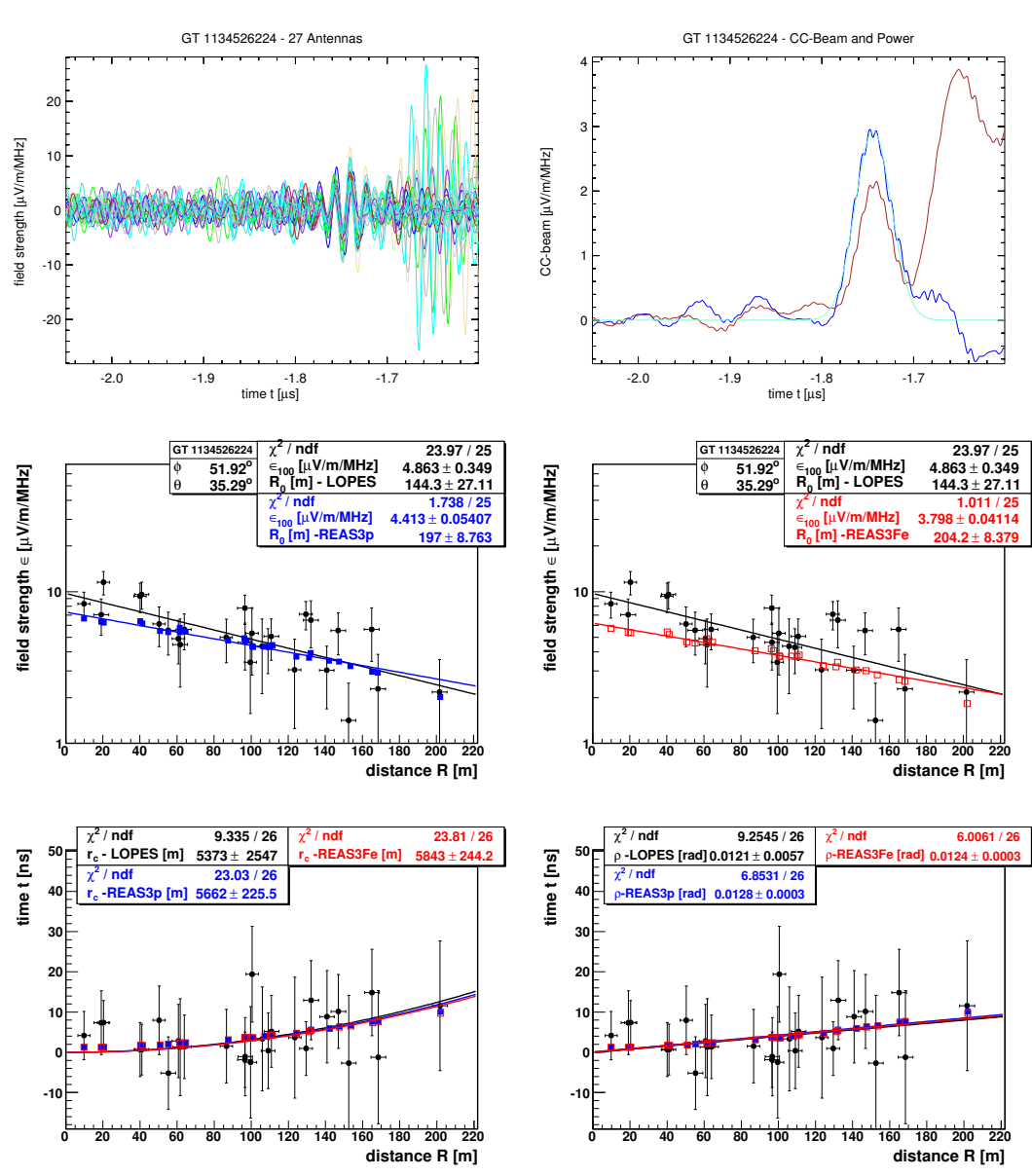

Figure D.1.: Event GT 1134526224 (LOPES 30, EW, KASCADE): $\theta=34.6^{\circ}, \phi=53.7^{\circ}, \alpha=52.8^{\circ}, E=0.12 \mathrm{EeV}$, $\ln A=3.9$
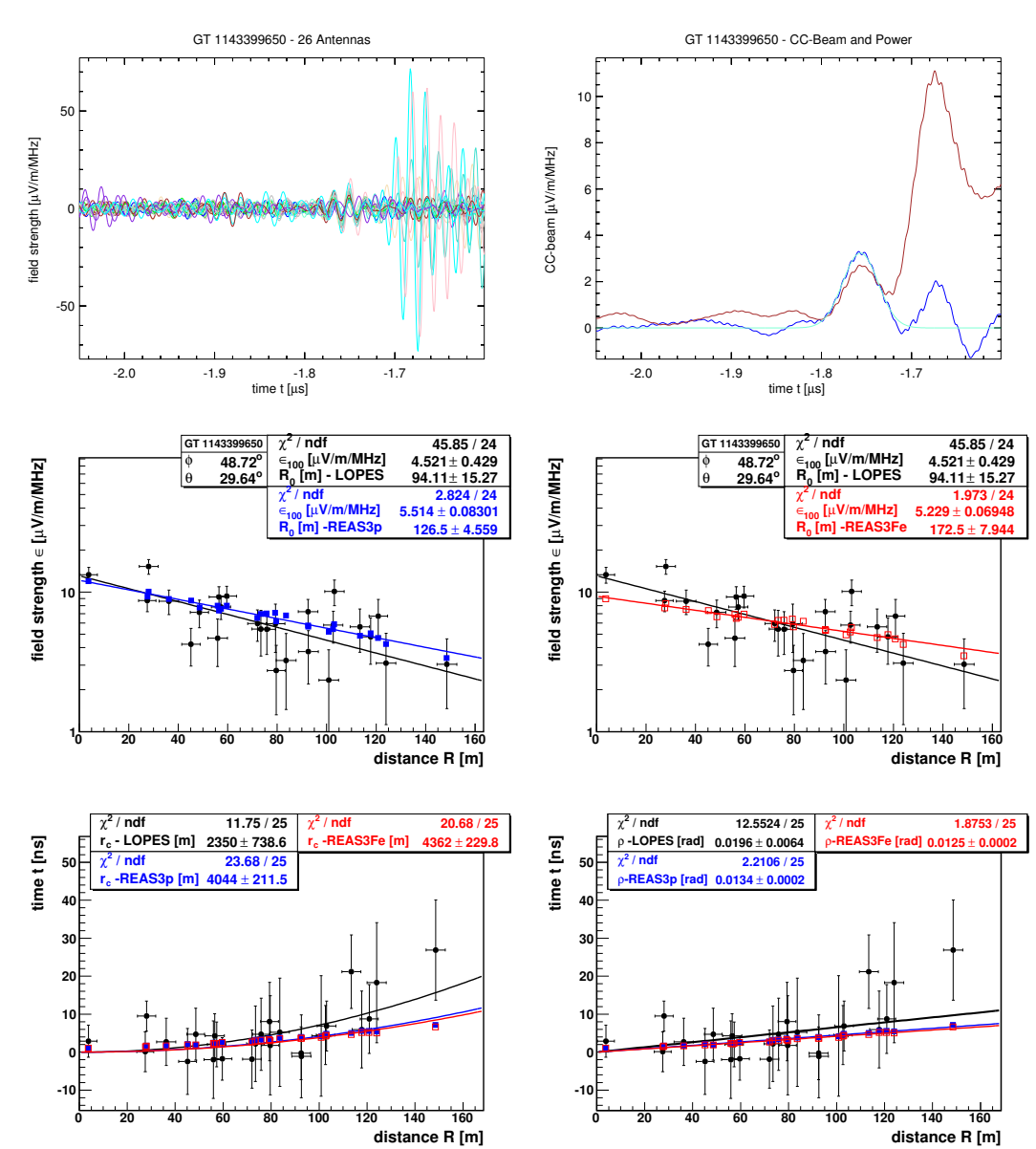

Figure D.2.: Event GT 1143399650 (LOPES 30, EW, KASCADE): $\theta=29.8^{\circ}, \phi=48.8^{\circ}, \alpha=49.6^{\circ}, E=0.15 \mathrm{EeV}$, $\ln A=3.3$ 

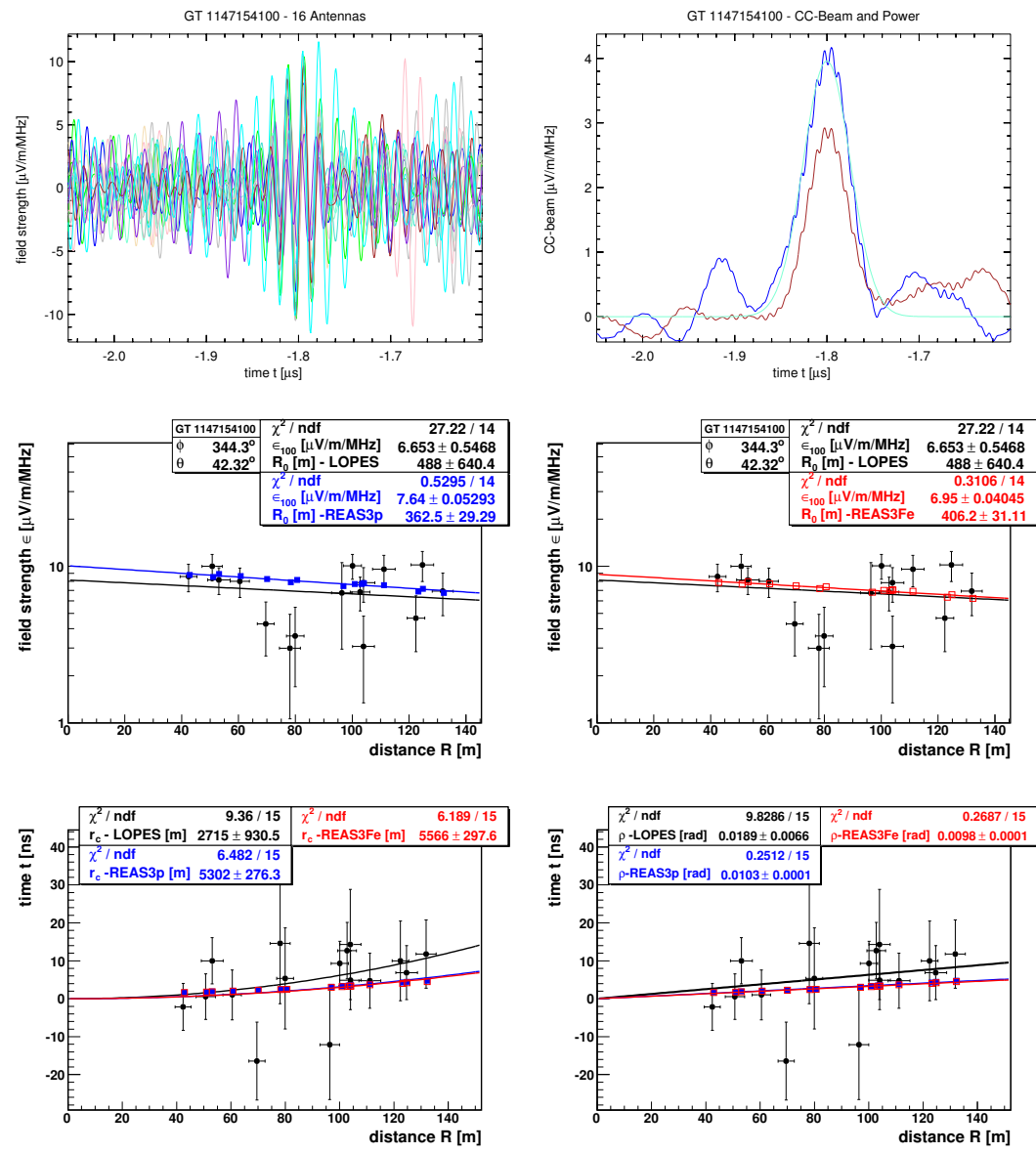

Figure D.3.: Event GT 1147154100 (LOPES 30, EW, KASCADE): $\theta=41.7^{\circ}, \phi=344.5^{\circ}, \alpha=66.0^{\circ}, E=0.16 \mathrm{EeV}$ $\ln A=4.7$
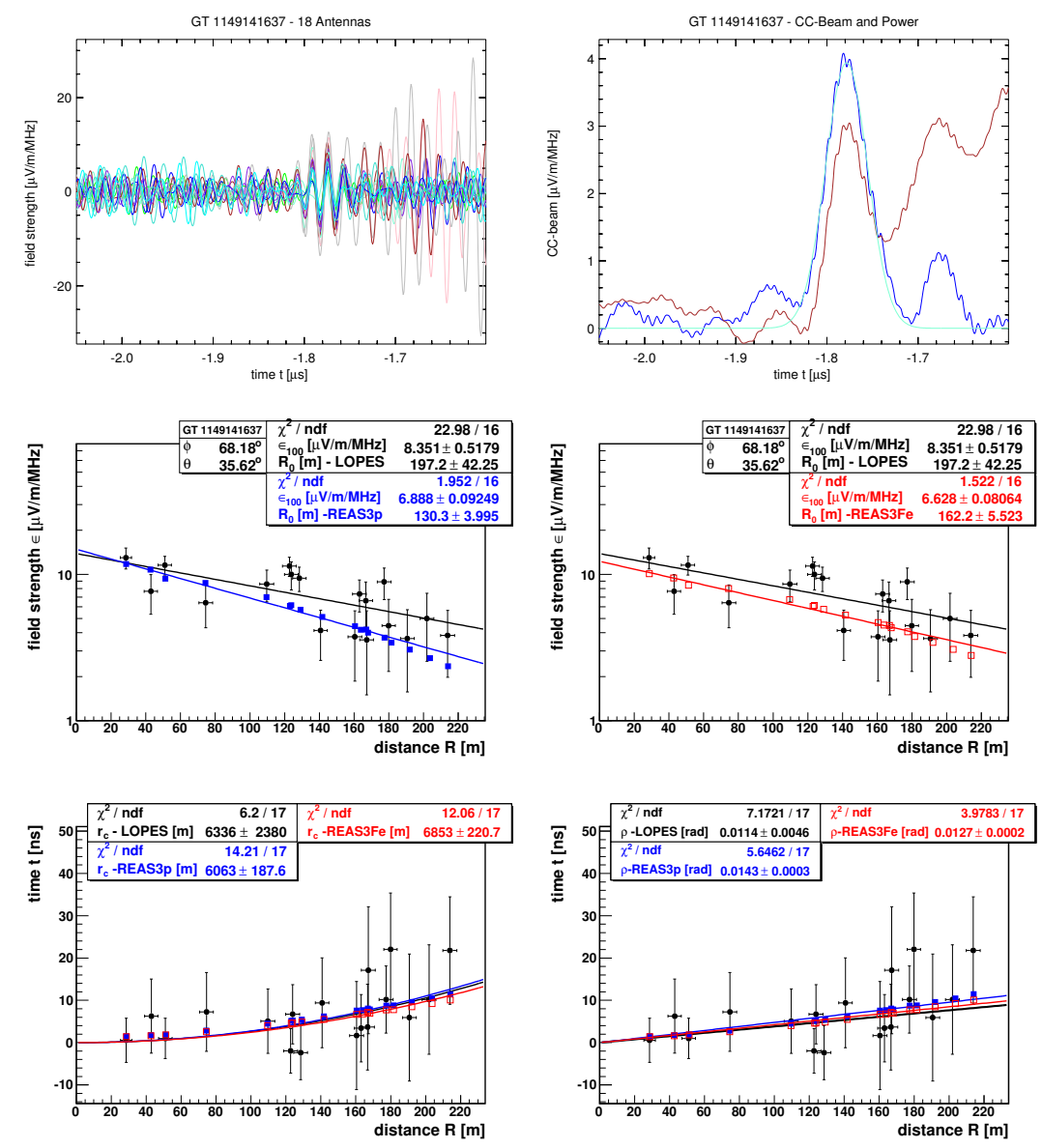

Figure D.4.: Event GT 1149141637 (LOPES 30, EW, KASCADE): $\theta=35.1^{\circ}, \phi=66.8^{\circ}, \alpha=49.8^{\circ}, E=0.24 \mathrm{EeV}$, $\ln A=4.4$ 

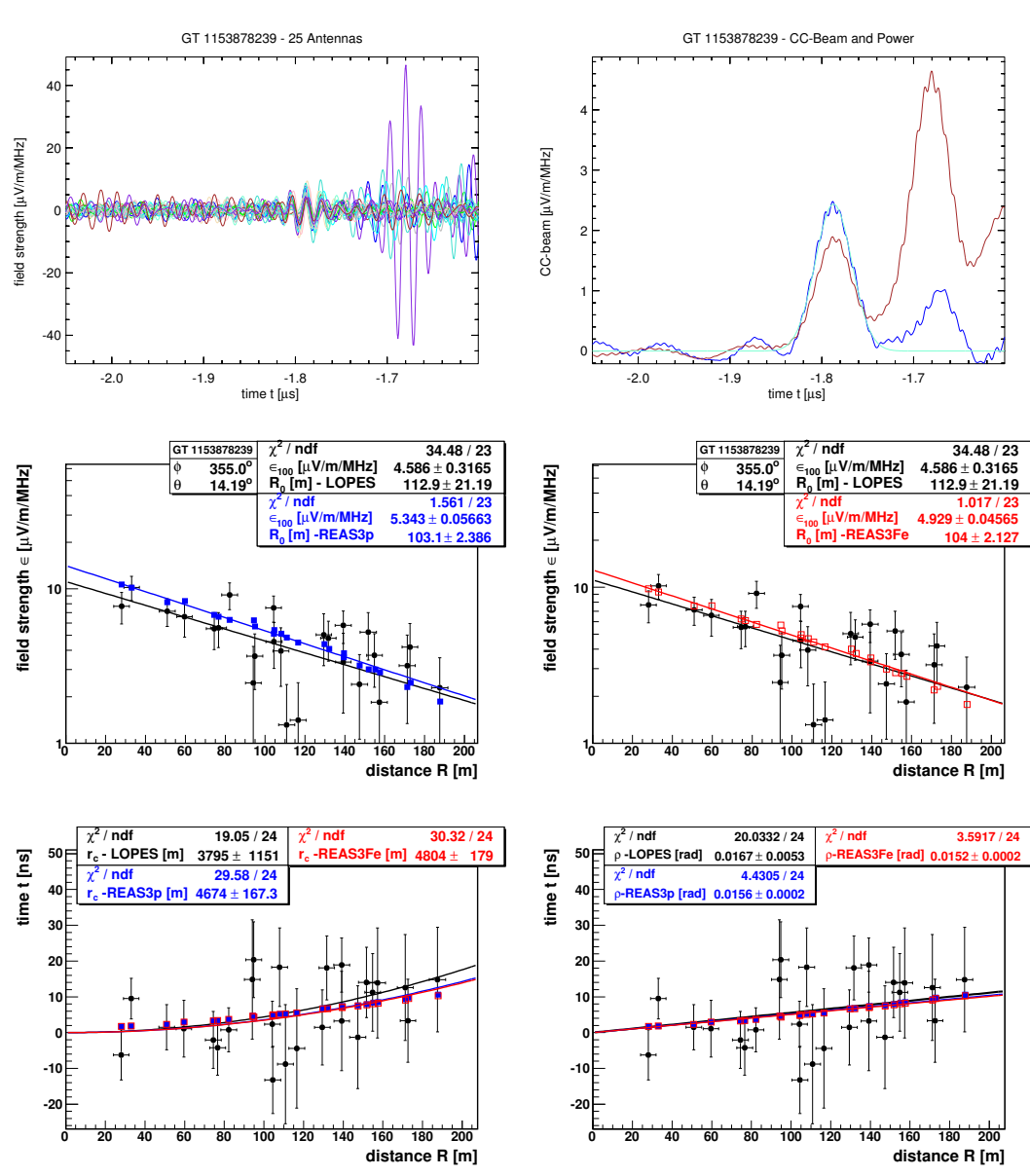

Figure D.5.: Event GT 1153878239 (LOPES 30, EW, KASCADE) $\theta=13.5^{\circ}, \phi=354.4^{\circ}, \alpha=38.5^{\circ}, E=0.17 \mathrm{EeV}$ $\ln A=1.4$
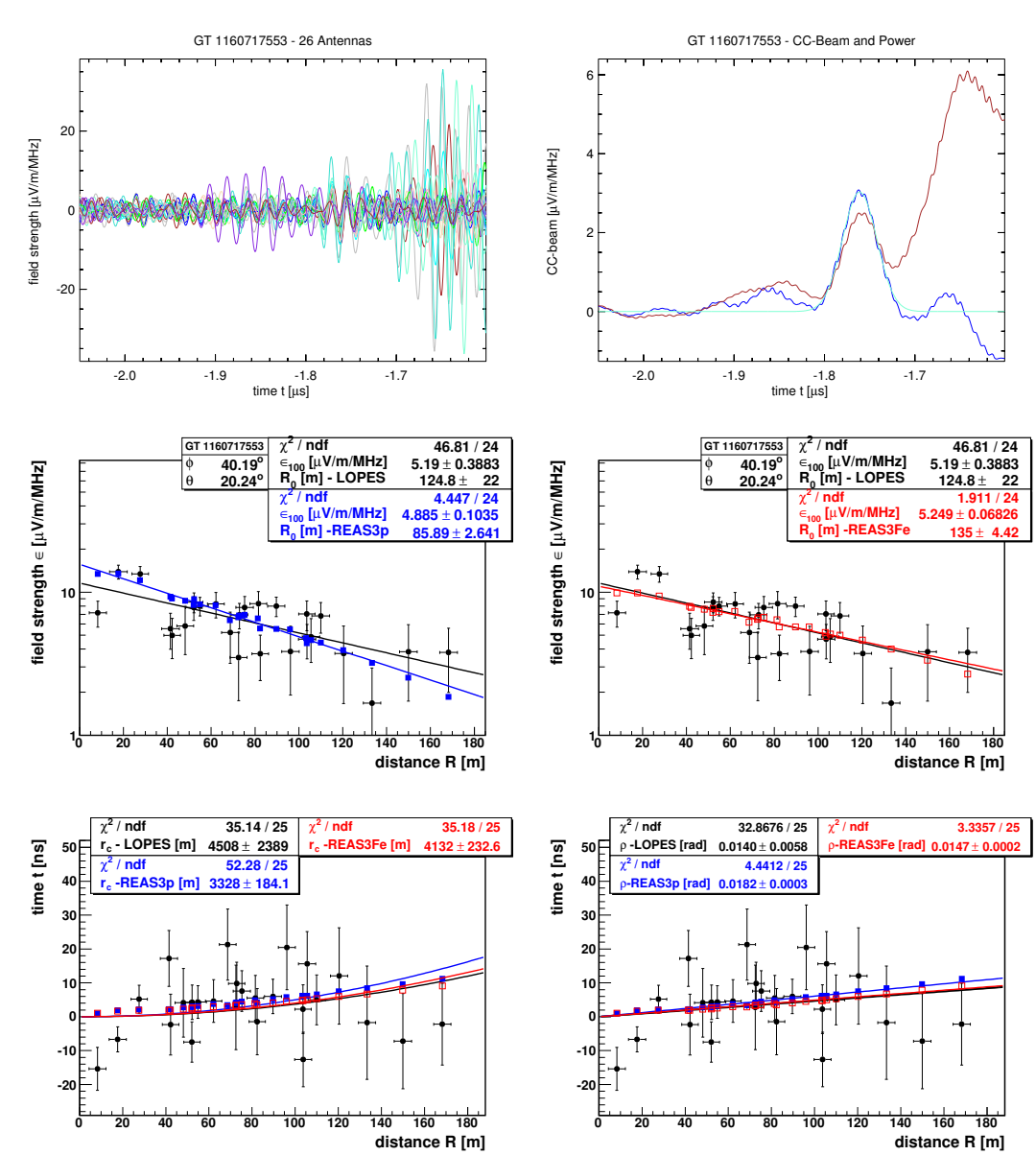

Figure D.6.: Event GT 1160717553 (LOPES 30, EW, KASCADE): $\theta=20.7^{\circ}, \phi=41.1^{\circ}, \alpha=42.7^{\circ}, E=0.16 \mathrm{EeV}$ $\ln A=2.3$ 

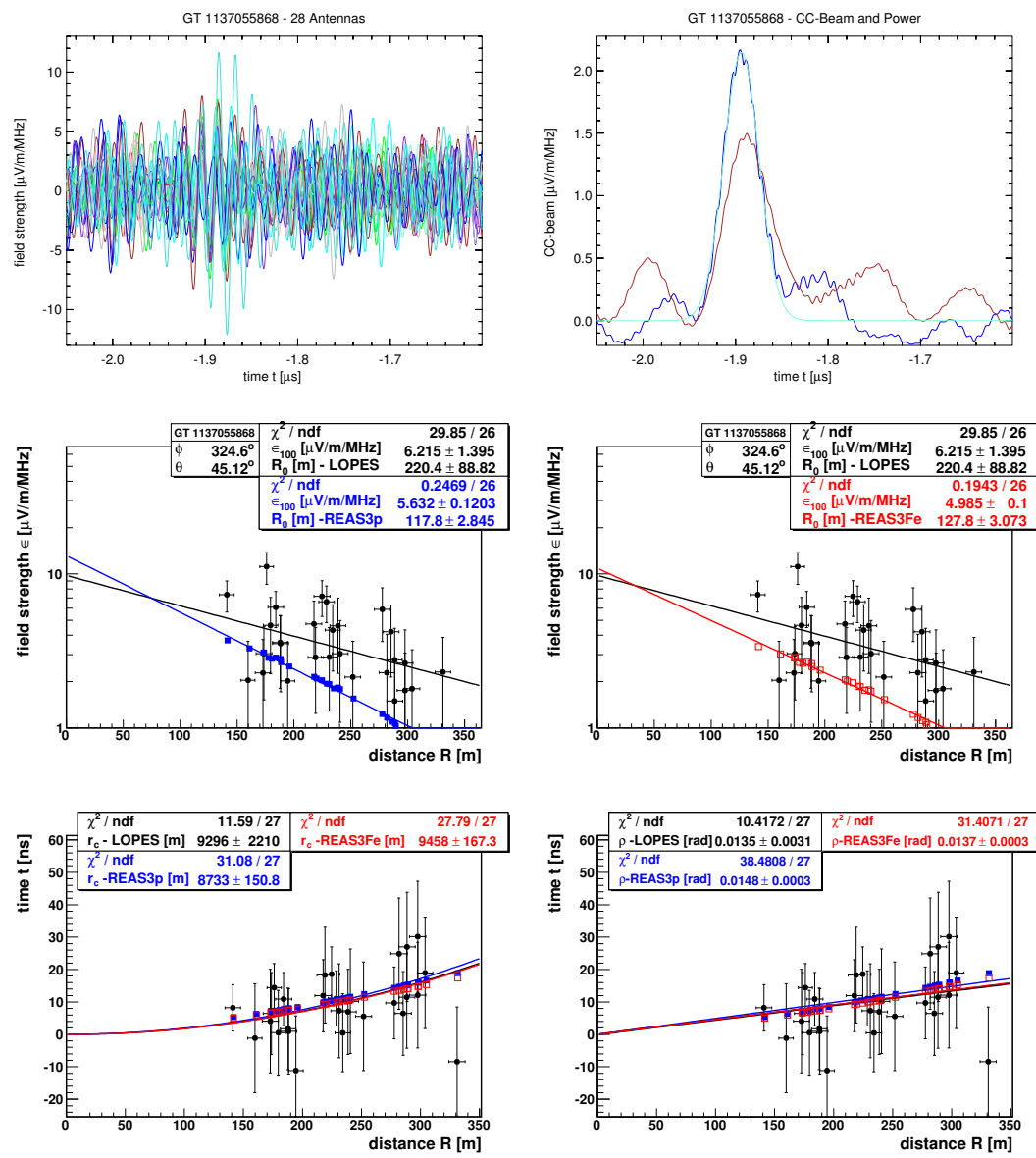

Figure D.7.: Event GT 1137055868 (LOPES 30, EW, Grande)
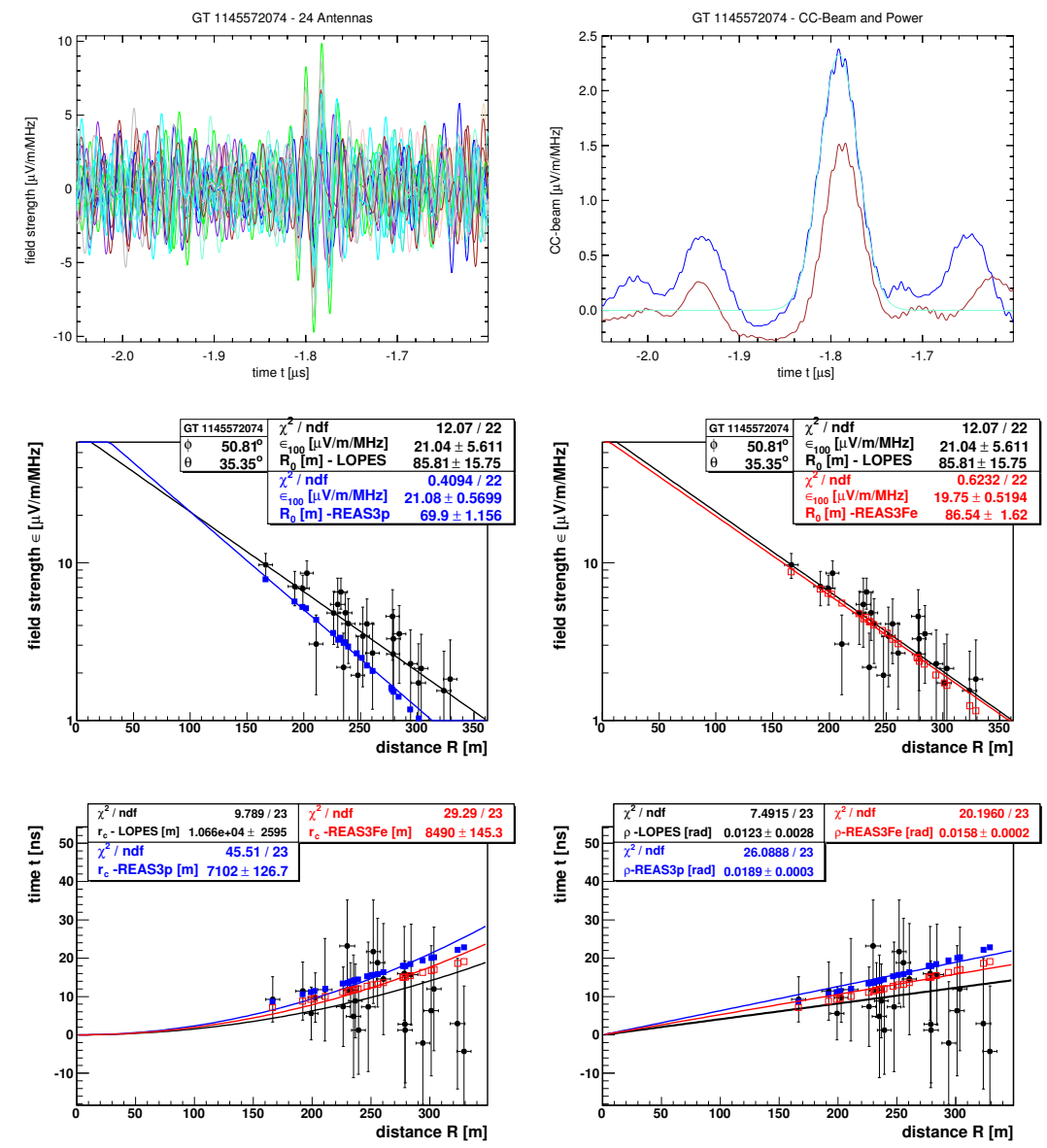

Figure D.8.: Event GT 1145572074 (LOPES 30, EW, Grande): $\theta=35.6^{\circ}, \phi=51.8^{\circ}, \alpha=54.2^{\circ}, E=0.37 \mathrm{EeV}$ $\ln A=2.5$ 

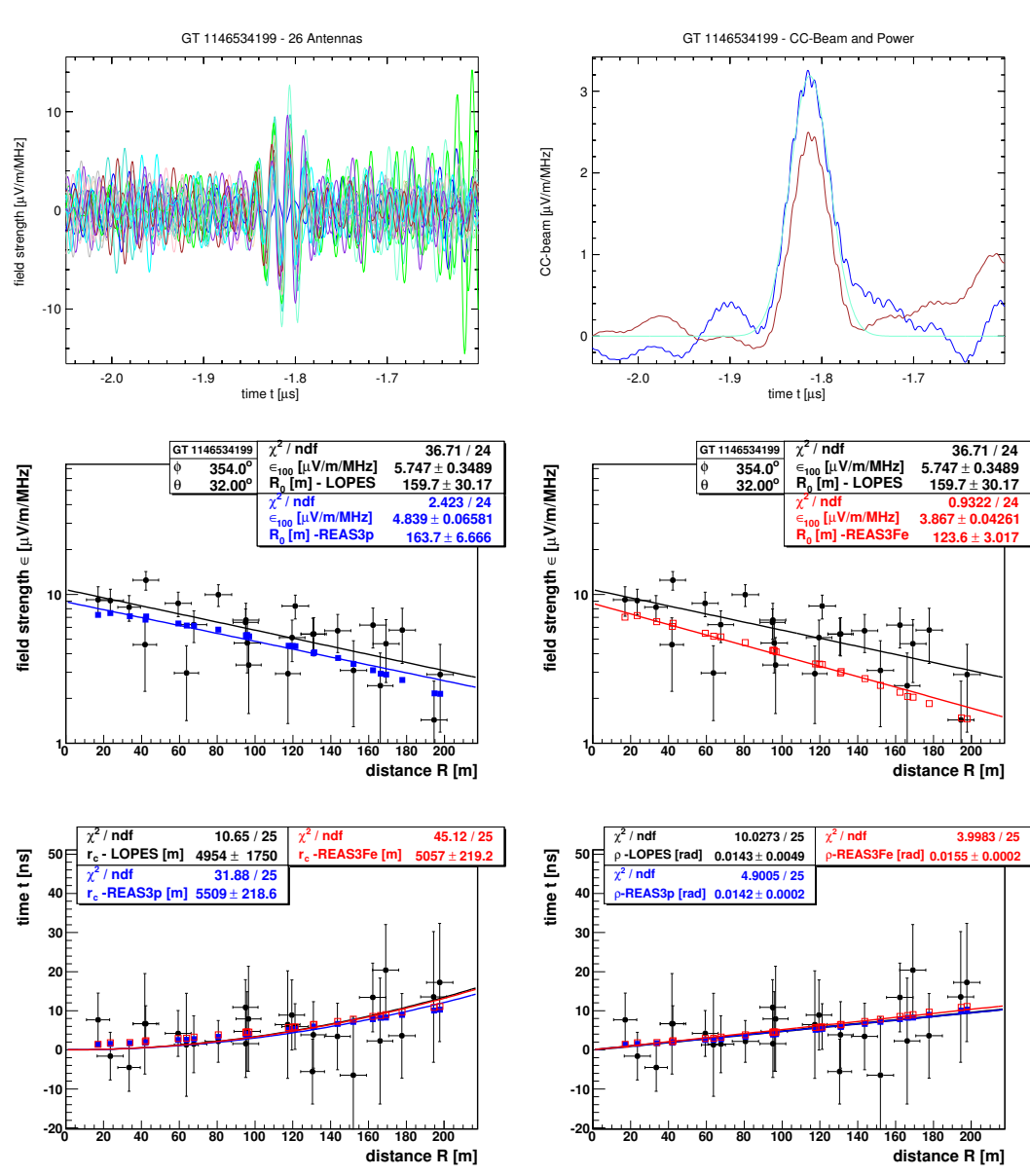

Figure D.9.: Event GT 1146534199 (LOPES 30, EW, Grande): $\theta=31.4^{\circ}, \phi=354.1^{\circ}, \alpha=56.3^{\circ}, E=0.10 \mathrm{EeV}$ $\ln A=4.1$
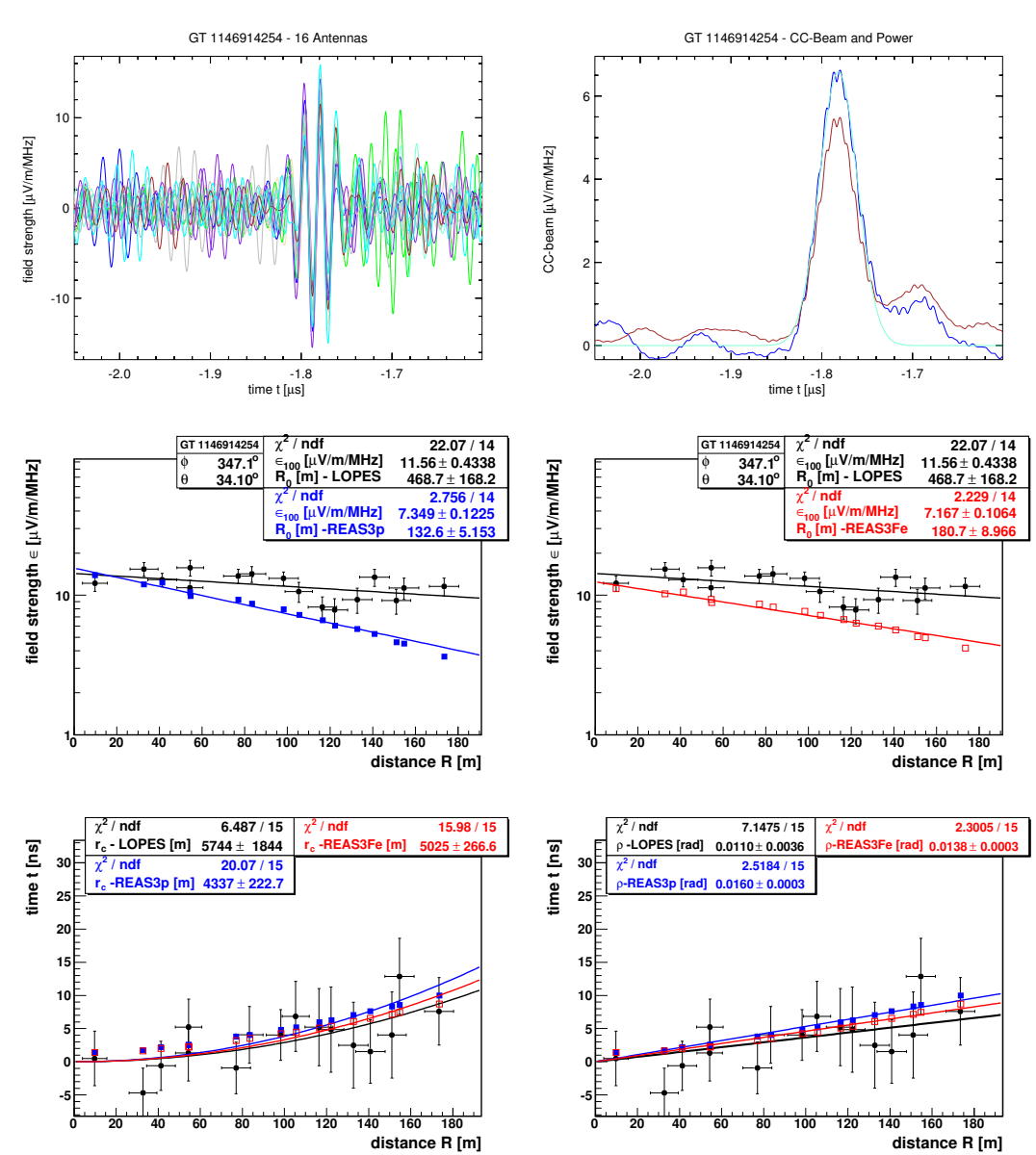

Figure D.10.: Event GT 1146914254 (LOPES 30, EW, Grande): $\theta=33.7^{\circ}, \phi=347.2^{\circ}, \alpha=58.3^{\circ}, E=0.17 \mathrm{EeV}$, $\ln A=4.9$ 

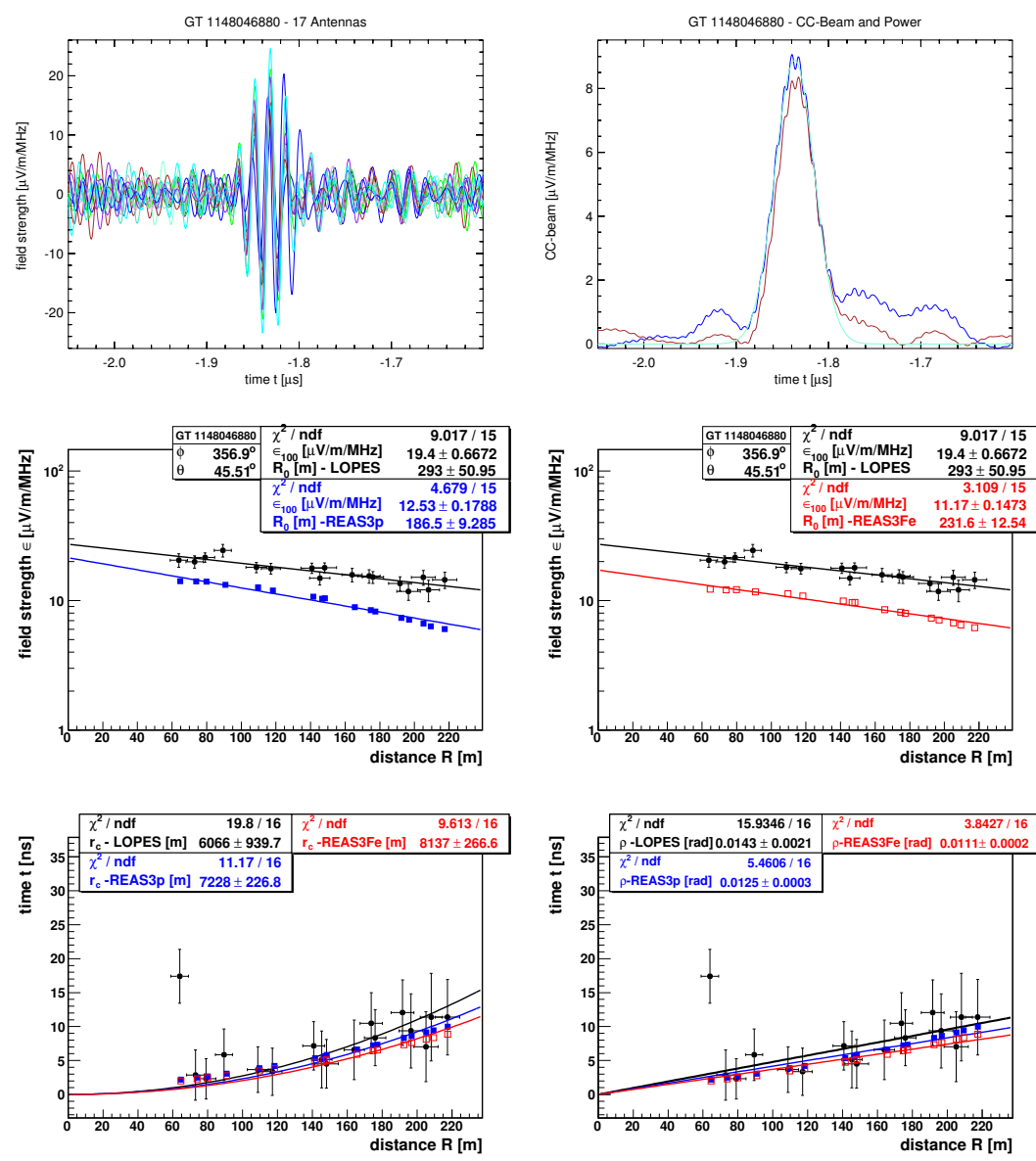

Figure D.11.: Event GT 1148046880 (LOPES 30, EW, Grande):
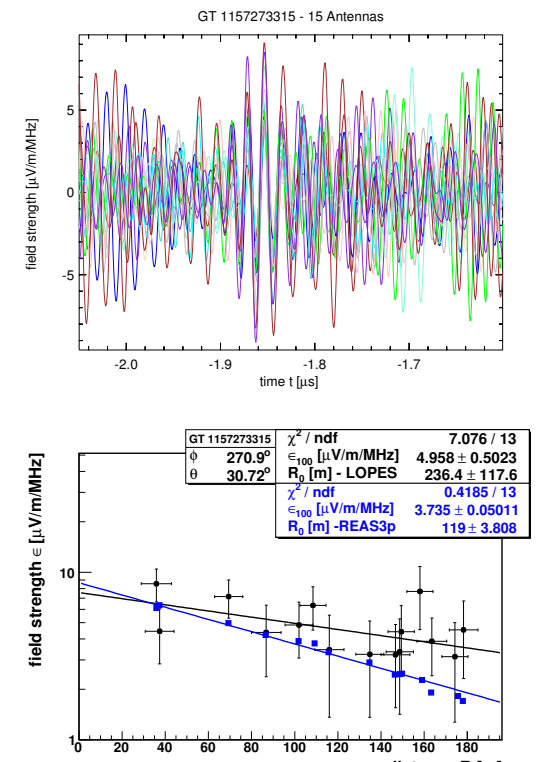

160
distance $R$ [m]
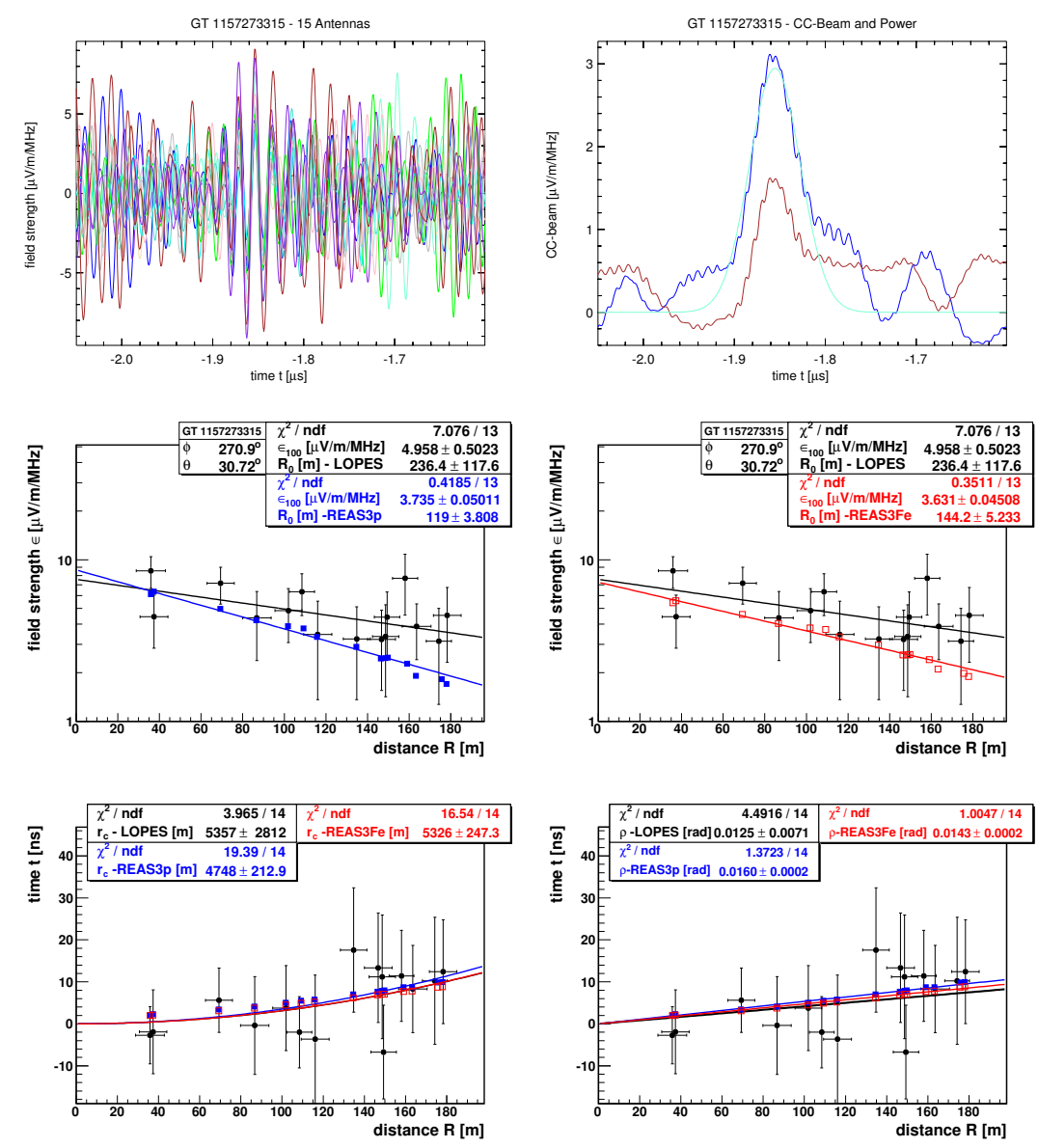

Figure D.12.: Event GT 1157273315 (LOPES 30, EW, Grande): $\theta=30.1^{\circ}, \phi=268.8^{\circ}, \alpha=37.9^{\circ}, E=0.17 \mathrm{EeV}$, $\ln A=4.2$ 

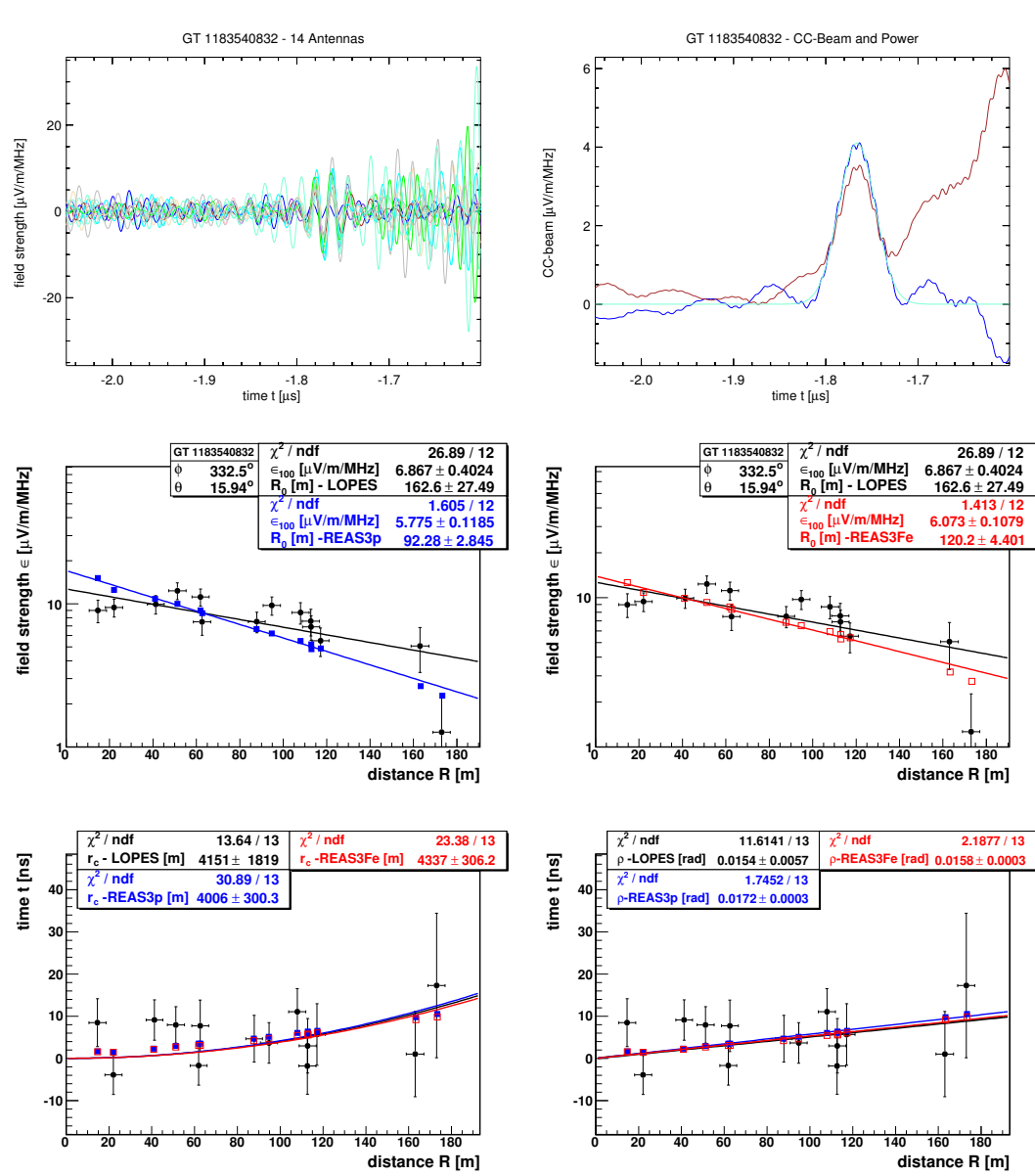

Figure D.13.: Event GT 1183540832 (LOPES Dual, EW, KASCADE) $\theta=15.5^{\circ}, \phi=336.5^{\circ}, \alpha=39.6^{\circ}, E=0.18 \mathrm{EeV}$, $\ln A=2.5$
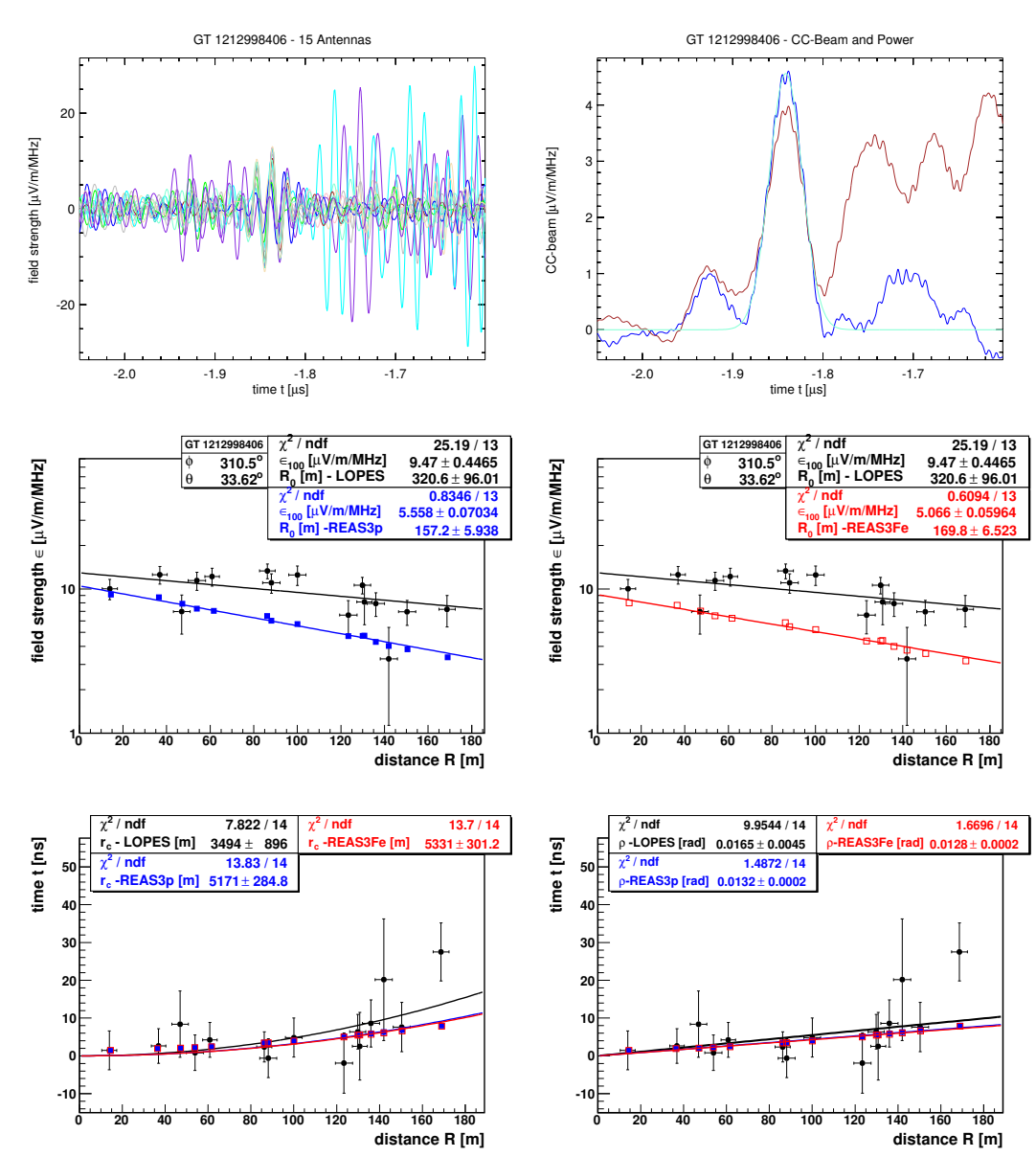

Figure D.14.: Event GT 1212998406 (LOPES Dual, EW, KASCADE): $\theta=33.3^{\circ}, \phi=309.4^{\circ}, \alpha=52.4^{\circ}, E=0.15 \mathrm{EeV}$, $\ln A=6.8$ 

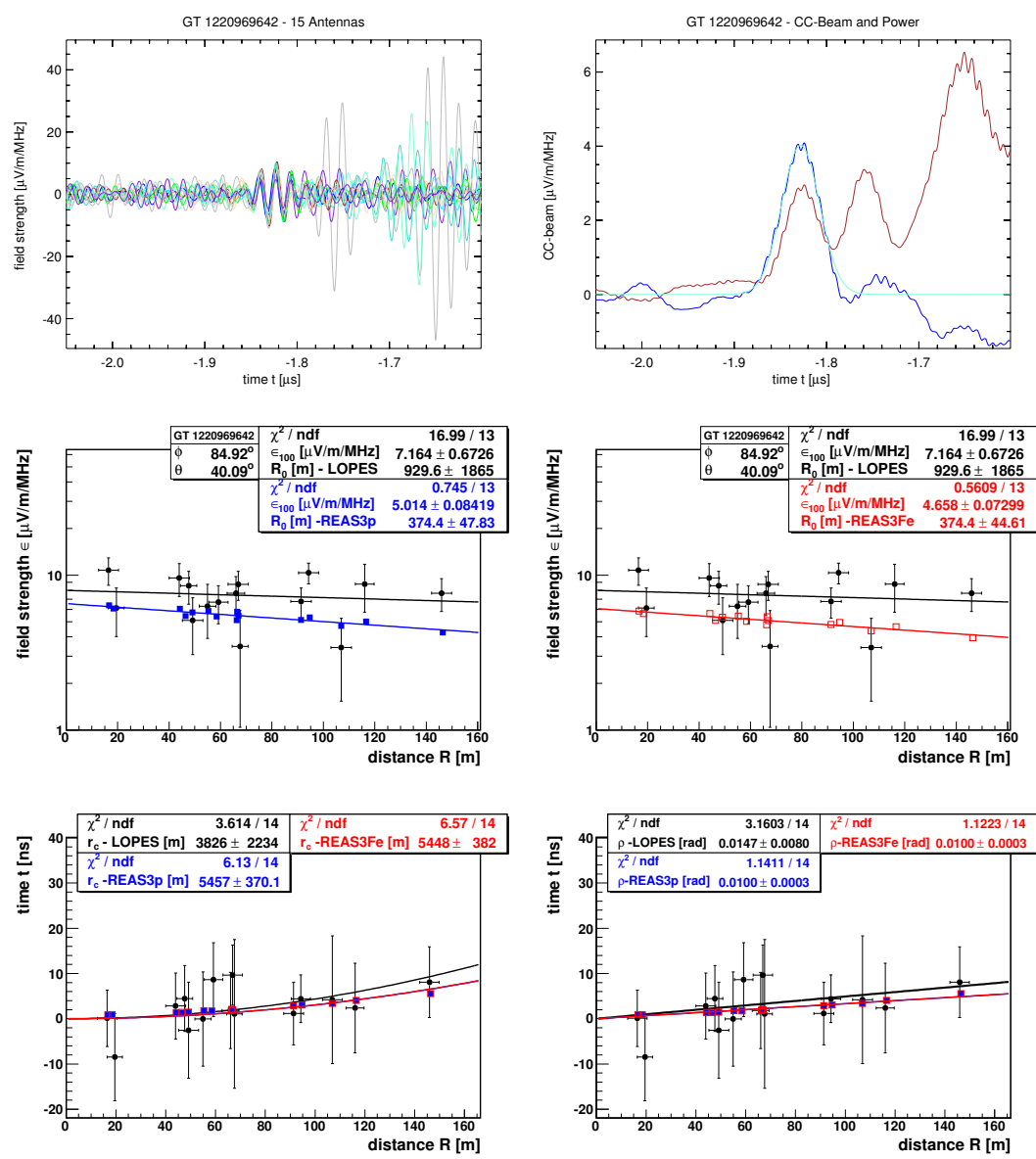
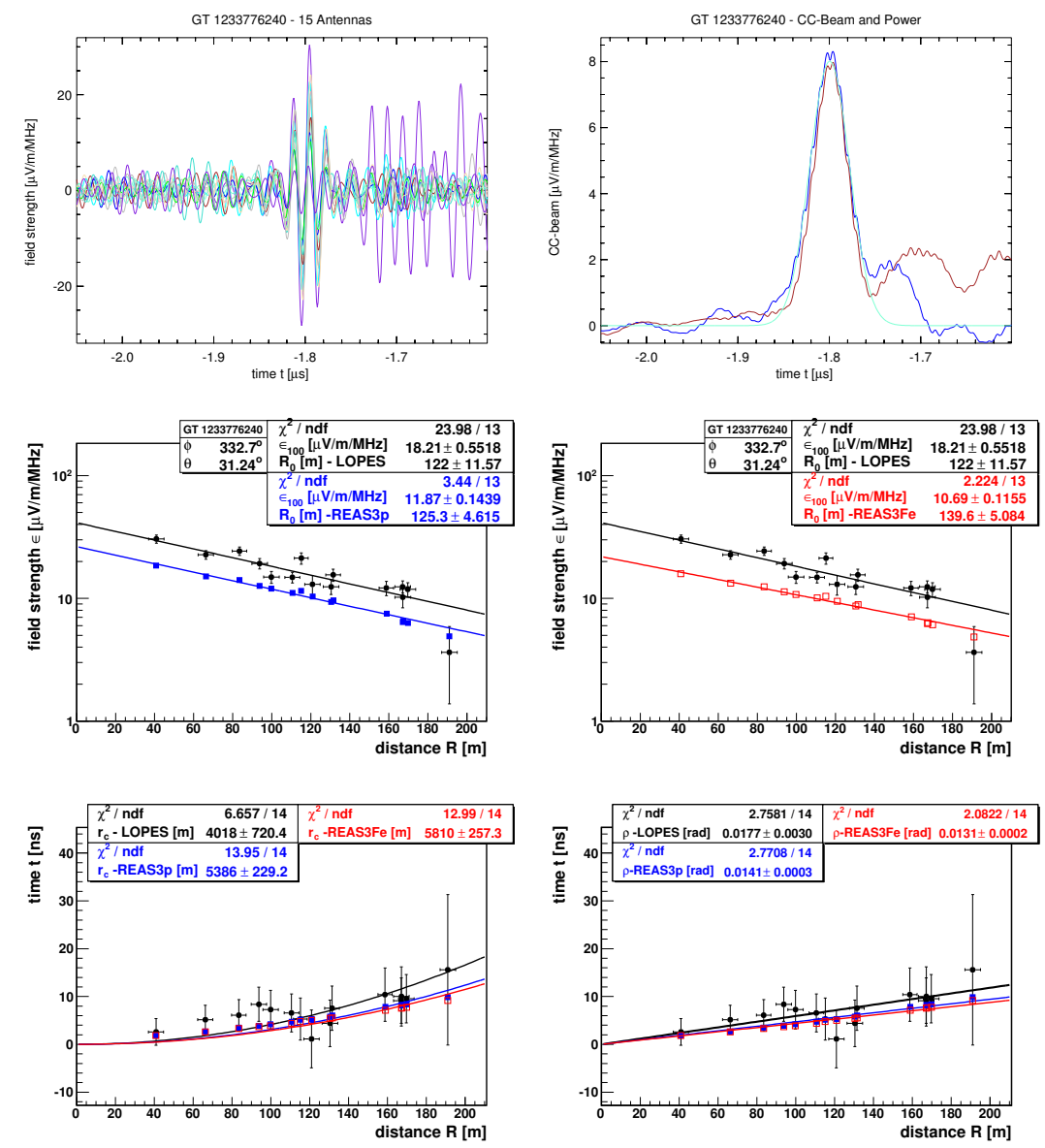

Figure D.16.: Event GT 1233776240 (LOPES Dual, EW, KASCADE): $\theta=30.9^{\circ}, \phi=331.1^{\circ}, \alpha=54.1^{\circ}, E=0.28 \mathrm{EeV}$, $\ln A=2.5$
Figure D.15.: Event GT 1220969642 (LOPES Dual, EW, KASCADE): $\theta=40.5^{\circ}, \phi=84.5^{\circ}, \alpha=48.5^{\circ}, E=0.23 \mathrm{EeV}$ $\ln A=4.9$ 

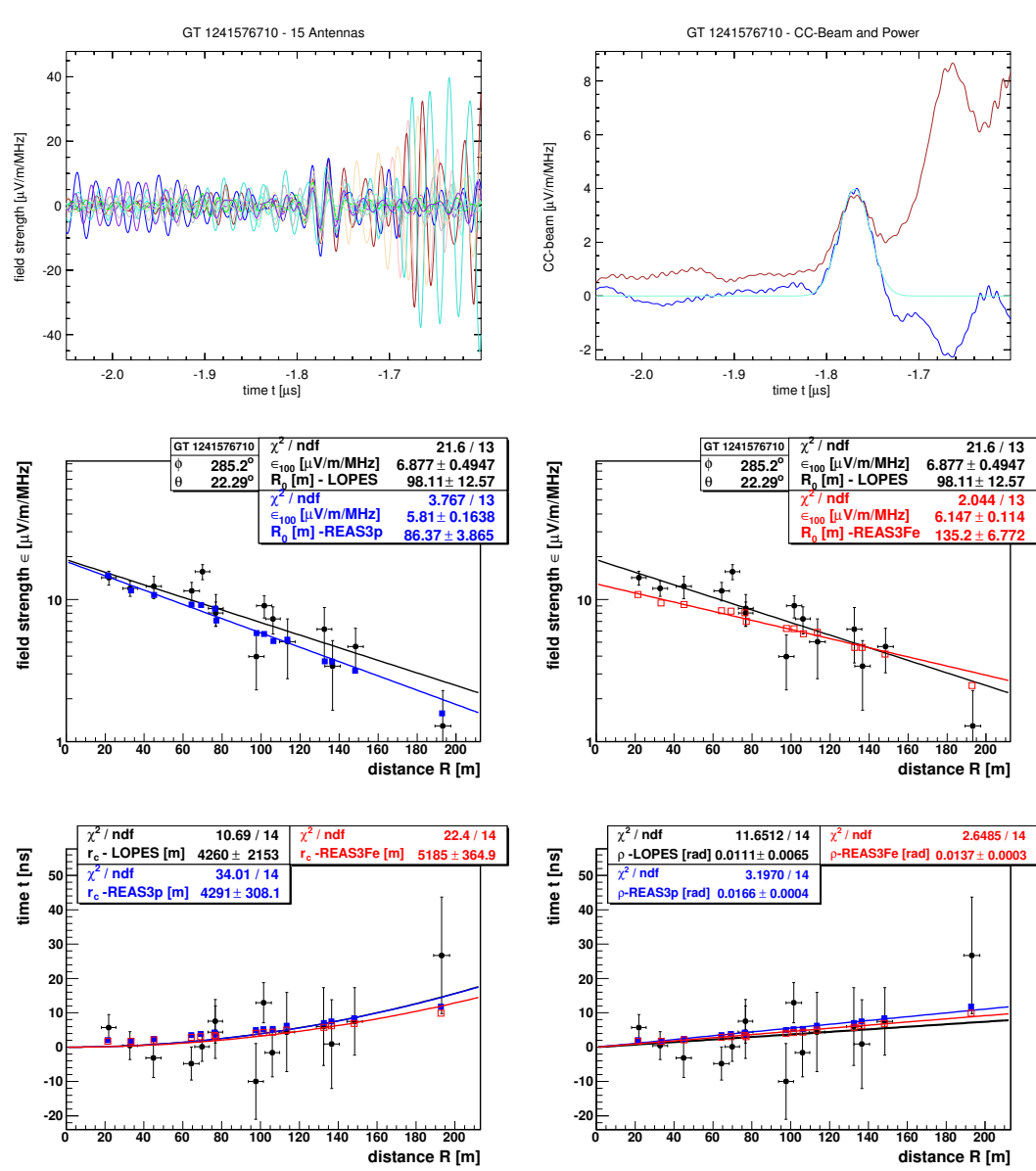
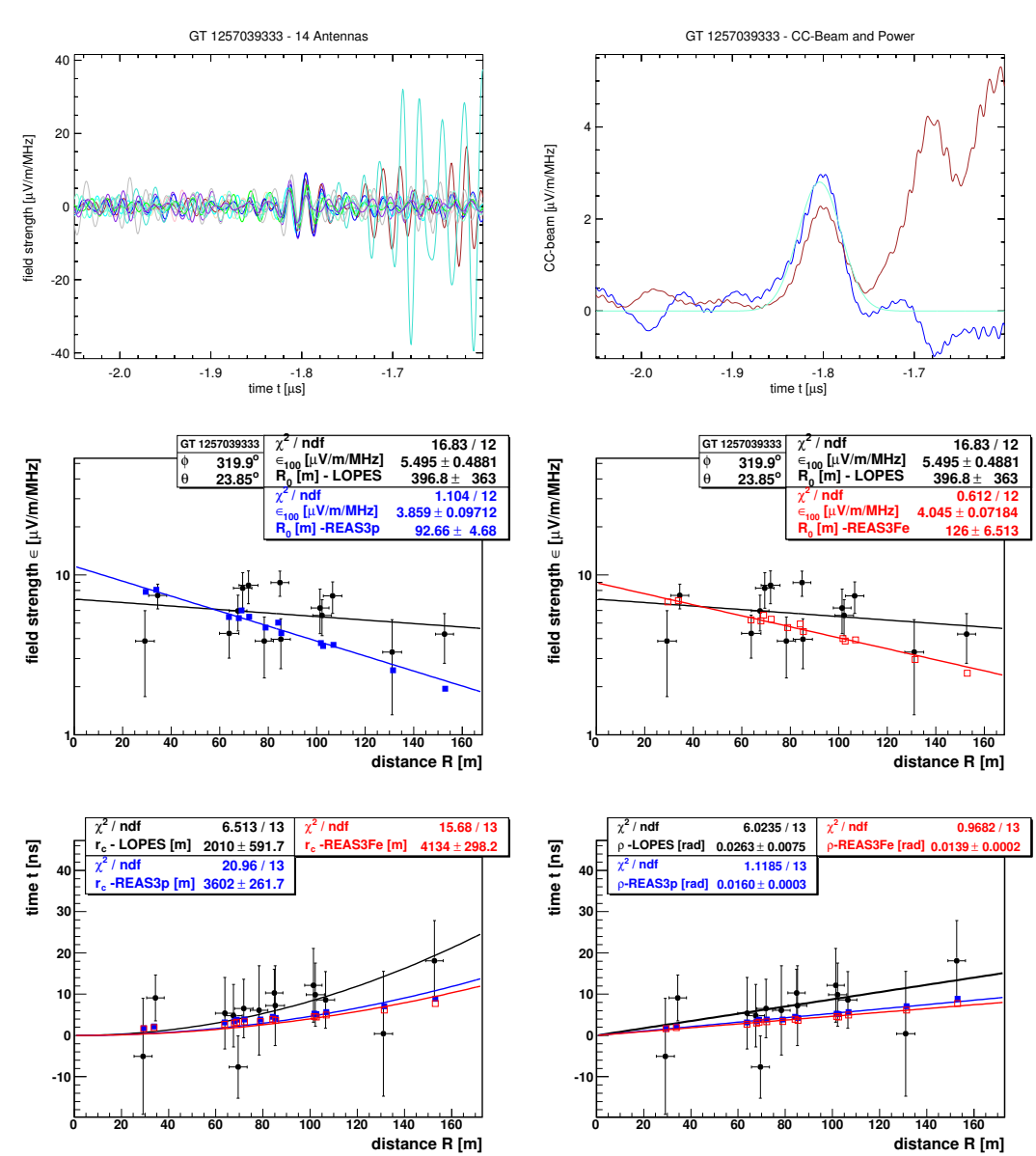

Figure D.17.: Event GT 1241576710 (LOPES Dual, EW, KASCADE): $\theta=22.5^{\circ}, \phi=287.3^{\circ}, \alpha=37.9^{\circ}, E=0.23 \mathrm{EeV}$, $\ln A=1.5$
Figure D.18.: Event GT 1257039333 (LOPES Dual, EW, KASCADE): $\theta=23.9^{\circ}, \phi=319.8^{\circ}, \alpha=45.8^{\circ}, E=0.12 \mathrm{EeV}$, $\ln A=2.9$ 

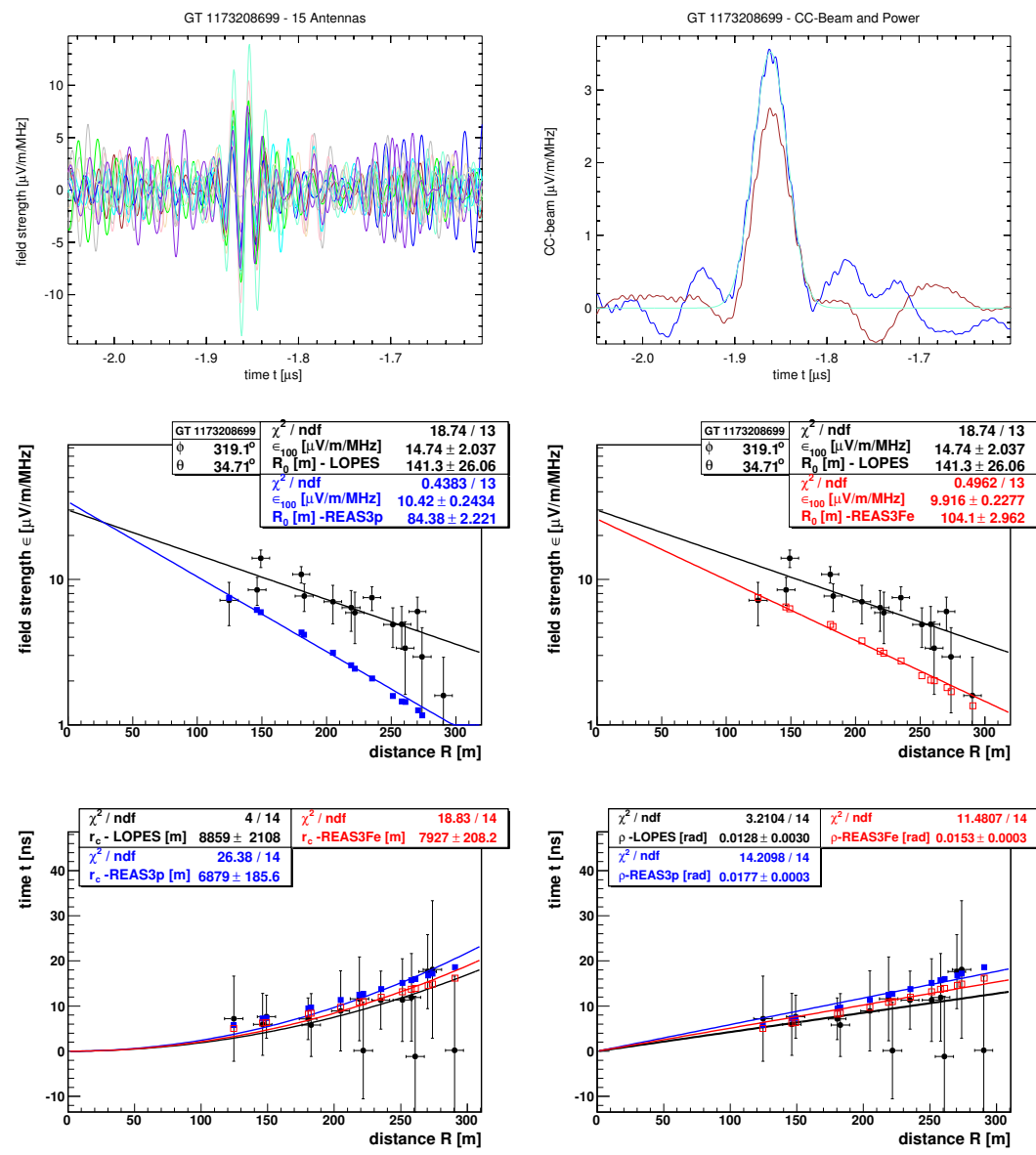

Figure D.19.: Event GT 1173208699 (LOPES Dual, EW, Grande): $\theta=34.1^{\circ}, \phi=319.2^{\circ}, \alpha=55.2^{\circ}, E=0.21 \mathrm{EeV}$ $\ln A=3.2$
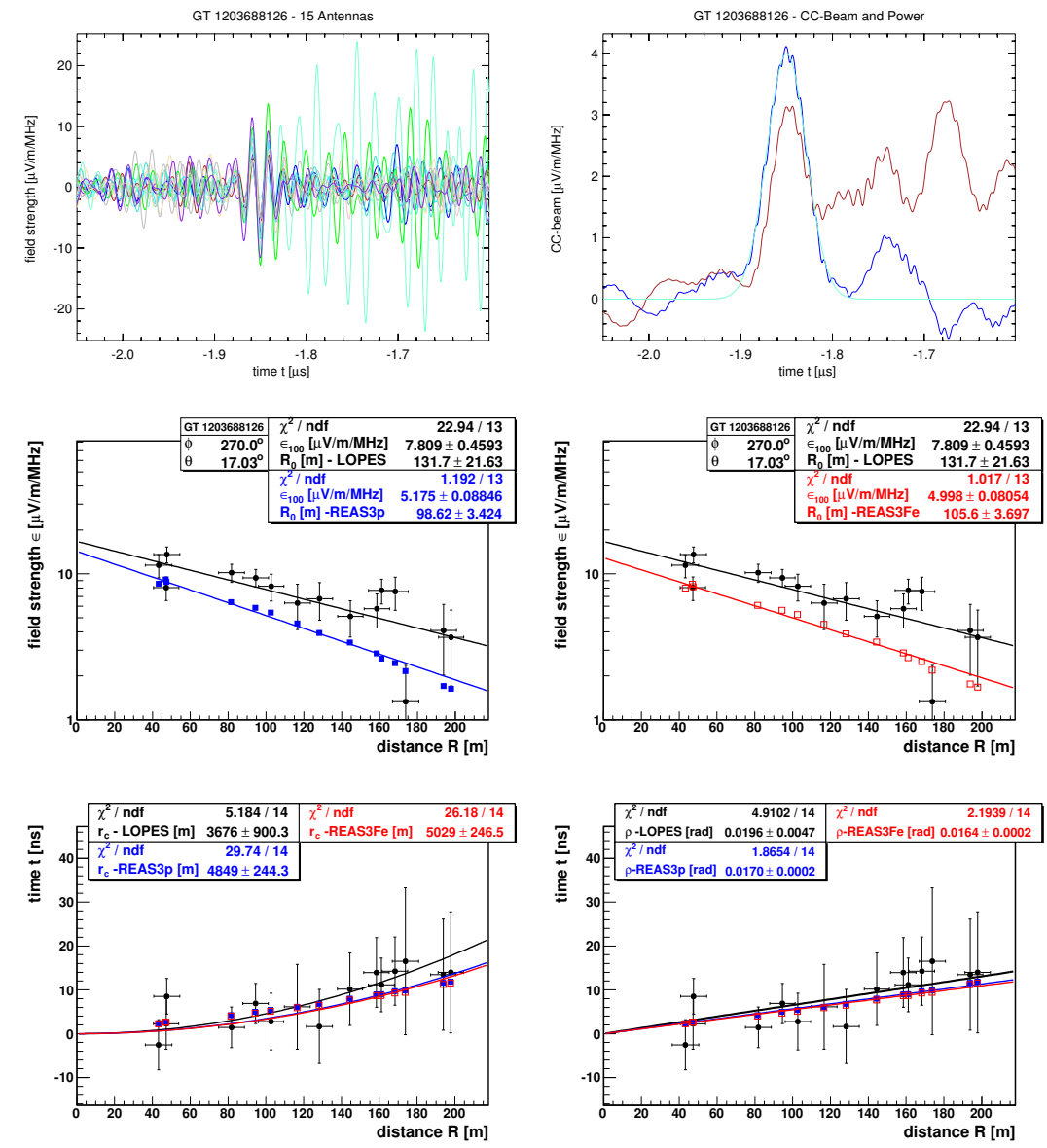

Figure D.20.: Event GT 1203688126 (LOPES Dual, EW, Grande): $\theta=16.7^{\circ}, \phi=269.8^{\circ}, \alpha=29.7^{\circ}, E=0.22 \mathrm{EeV}$, $\ln A=4.1$ 

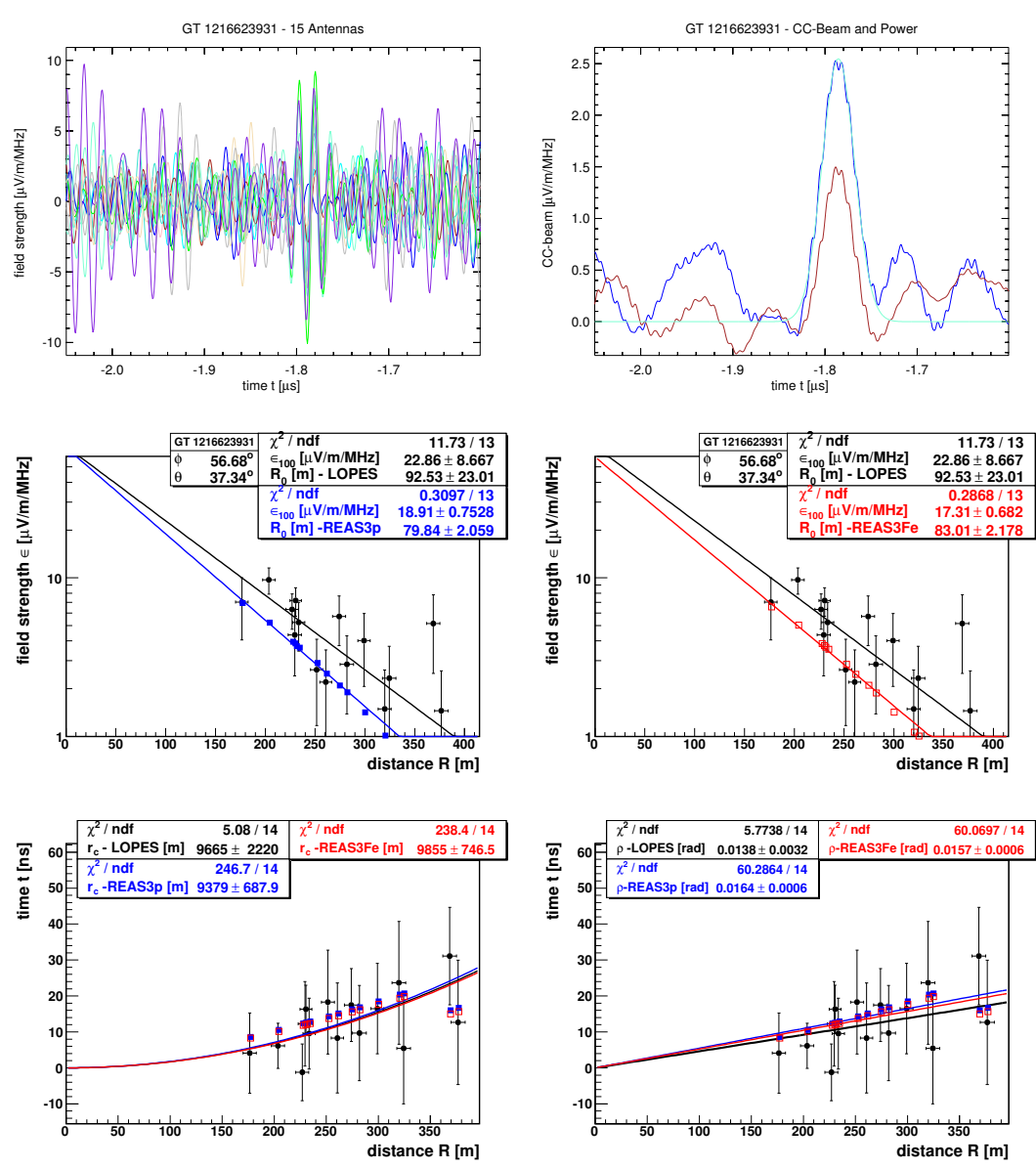

Figure D.21.: Event GT 1216623931 (LOPES Dual, EW, Grande): $\theta=37.1^{\circ}, \phi=56.8^{\circ}, \alpha=54.3^{\circ}, E=0.33 \mathrm{EeV}$ $\ln A=3.7$
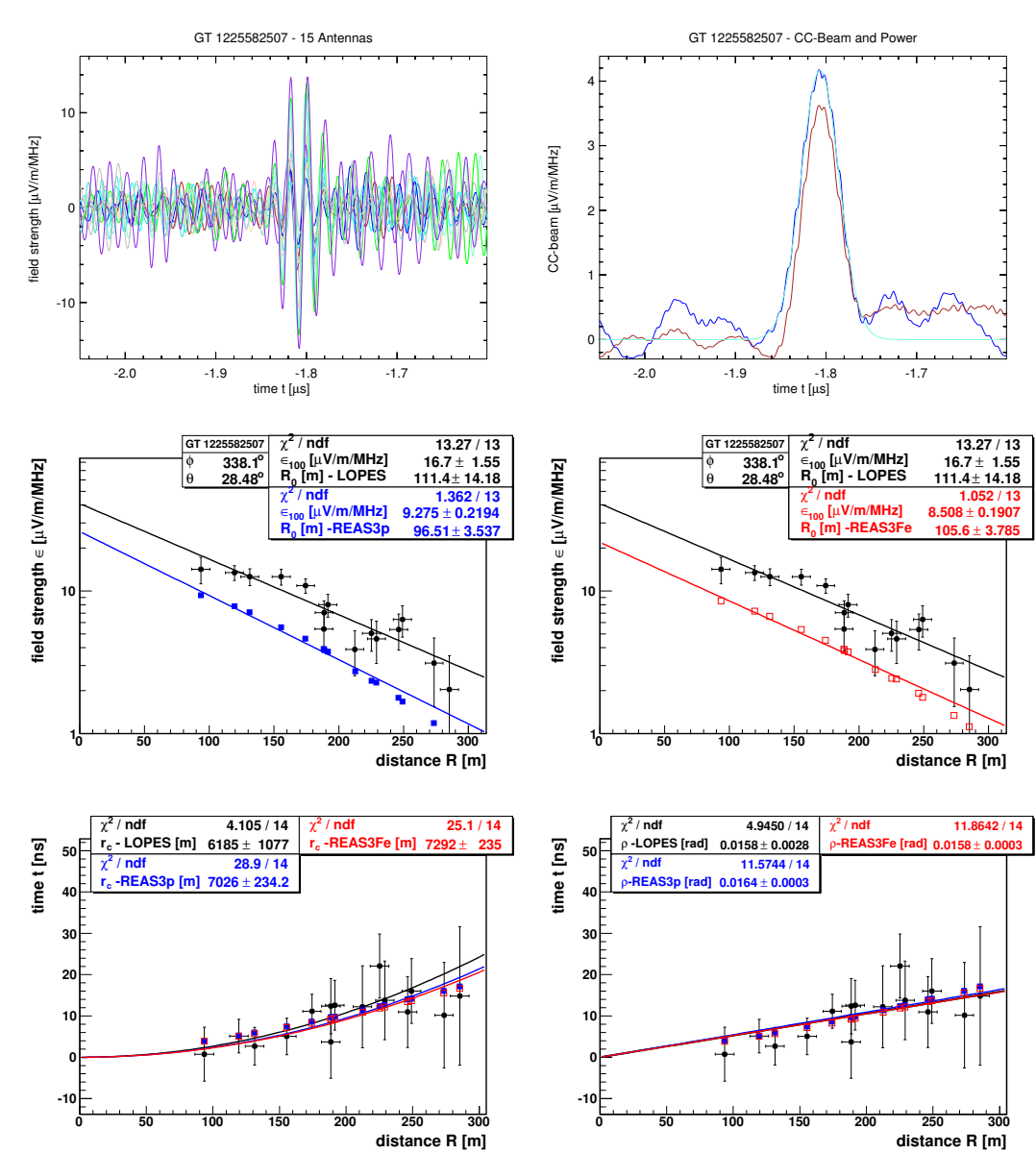

Figure D.22.: Event GT 1225582507 (LOPES Dual, EW, Grande): $\theta=28.2^{\circ}, \phi=338.1^{\circ}, \alpha=52.1^{\circ}, E=0.19 \mathrm{EeV}$, $\ln A=2.9$ 

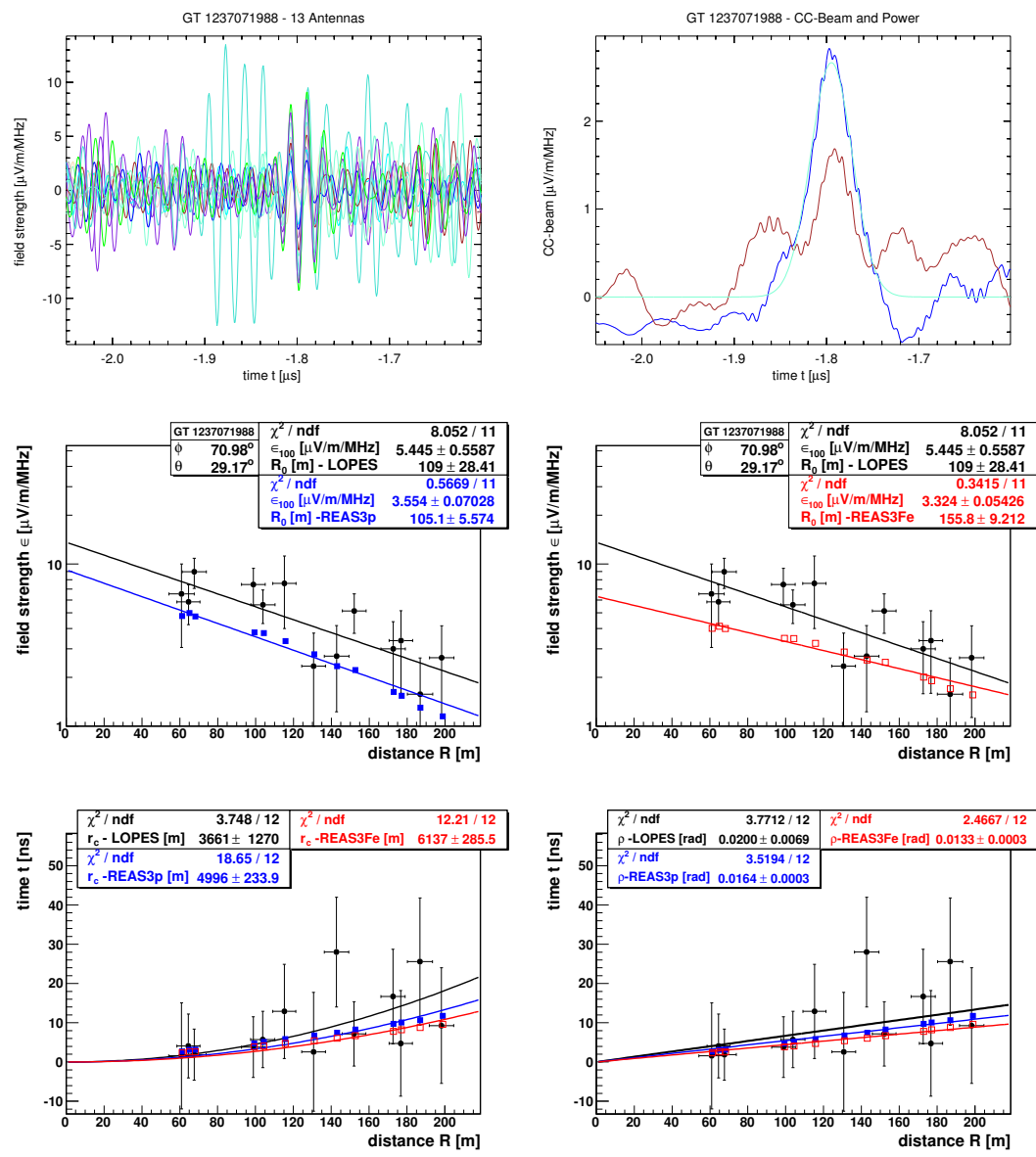

Figure D.23.: Event GT 1237071988 (LOPES Dual, EW, Grande): $\theta=29.2^{\circ}, \phi=70.7^{\circ}, \alpha=43.7^{\circ}, E=0.12 \mathrm{EeV}$ $\ln A=1.2$
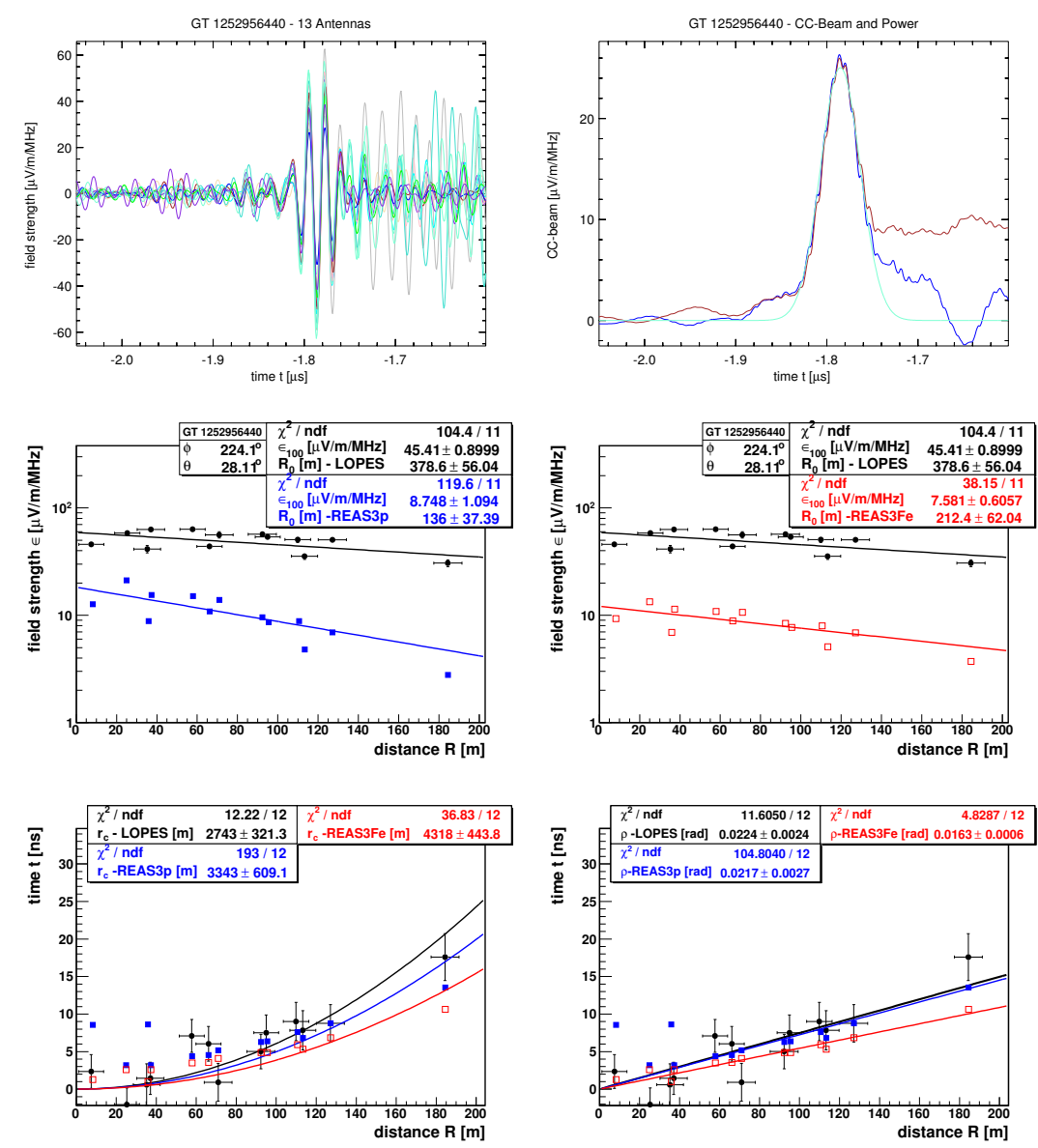

Figure D.24.: Event GT 1252956440 (LOPES Dual, EW, Grande): $\theta=26.9^{\circ}, \phi=222.6^{\circ}, \alpha=18.4^{\circ}, E=1.11 \mathrm{EeV}$, $\ln A=0.7$ 



\section{Bibliography}

[1] N. Jarosik et al. - WMAP. Seven-Year Wilkinson Microwave Anisotropy Probe (WMAP) Observations: Sky Maps, Systematic Errors, and Basic Results. ArXiv e-prints, 2010. astro-ph/1001.4744.

[2] J. Linsley. Evidence for a Primary Cosmic-Ray Particle with Energy $10^{20} \mathrm{eV}$. Physical Review Letters, 10(4):146-148, 1963.

[3] V. F. Hess. Über Beobachtungen der durchdringenden Strahlung bei sieben Freiballonfahrt. Physikalische Zeitschrift, 13:1084, 1912.

[4] J. Blümer, R. Engel, and J. R. Hörandel. Cosmic rays from the knee to the highest energies. Progress in Particle and Nuclear Physics, 63:293-338, 2009.

[5] E. Fermi. On the Origin of Cosmic Radiation. Physical Review, 75(8):1169, 1949.

[6] W. Axford et al. In Proceedings of the 15th ICRC, Plovidv, Bulgaria, volume 11, page 132, 1977.

[7] R. Cowsik and L.W. Wilson. In Proceedings of the 13th ICRC, Denver, USA, volume 1, page 500, 1973.

[8] F. Aharonian et al. - H.E.S.S. Collaboration. Primary particle acceleration above $100 \mathrm{TeV}$ in the shell-type supernova remnant RX J1713.7-3946h deep HESS observations. Astronomy \& Astrophysics, 464(1):235-243, 2007.

[9] B. F. Rauch et al. - TIGER Collaboration. Cosmic Ray origin in OB Associations and Preferential Acceleration of Refractory Elements: Evidence from Abundances of Elements ${ }_{26} \mathrm{Fe}$ through ${ }_{34} \mathrm{Se}$. Astrophysical Journal, 697:2083$2088,2009$.

[10] A. M. Hillas. The Origin of Ultra-High-Energy Cosmic Rays. Annual Review of Astronomy and Astrophysics, 22:425-444, 1984.

[11] W. D. Apel et al. - KASCADE Collaboration. Energy spectra of elemental groups of cosmic rays: Update on the KASCADE unfolding analysis. Astroparticle Physics, 31(2):86 - 91, 2009.

[12] W. D. Apel et al. - KASCADE-Grande Collaboration. The spectrum of highenergy cosmic rays measured with KASCADE-Grande. submitted to: Astroparticle Physics, 2010.

[13] J. Abraham et al. - Pierre Auger Observatory. Anisotropy studies around the galactic centre at EeV energies with the Auger Observatory. Astroparticle Physics, 27:244-253, 2007. 
[14] P. Abreu et al. - Pierre Auger Observatory. Update on the correlation of the highest energy cosmic rays with nearby extragalactic matter. Astroparticle Physics, 34:314-326, 2010.

[15] K. Greisen. End to the Cosmic-Ray Spectrum? Physical Review Letters, 16:748-750, 1966.

[16] G. T. Zatsepin and V. A. Kuzmin. Upper limit of the spectrum of cosmic rays. ZhETF Pis ma Redaktsiiu, 4(3):114-117, 1966.

[17] D. Allard, E. Parizot, A. V. Olinto, et al. UHE nuclei propagation and the interpretation of the ankle in the cosmic-ray spectrum. Astronomy $\&$ Astrophysics, 443(3):L29-L32, 2005.

[18] J. Abraham et al. - Pierre Auger Observatory. Properties and performance of the prototype instrument for the Pierre Auger Observatory. Nuclear Instruments and Methods in Physics Research A, 523(1-2):50 - 95, 2004.

[19] Y. Takahashi et al. - EUSO Collaboration. Science Objectives of the JEM EUSO mission on International Space Station. In Proceedings of the 30th ICRC, Merida, Mexico, 2007.

[20] A. Haungs, H. Rebel, and M. Roth. Energy spectrum and mass composition of high-energy cosmic rays. Reports on Progress in Physics, 66(7):1145, 2003.

[21] P. Auger, P. Ehrenfest, R. Maze, et al. Extensive Cosmic-Ray Showers. Reviews of Modern Physics, 11:288-291, 1939.

[22] S. J. Sciutto. AIRES: A system for air shower simulations (Version 2.2.0). ArXiv Astrophysics e-prints, 1999. astro-ph/9911331.

[23] D. Heck, J. Knapp, J. N. Capdevielle, et al. CORSIKA: A Monte Carlo Code to Simulate Extensive Air Showers. FZKA Report 6019, Forschungszentrum Karlsruhe, 1998.

[24] W. D. Apel et al. - KASCADE Collaboration. Test of interaction models up to $40 \mathrm{PeV}$ by studying hadronic cores of EAS. Journal of Physics G: Nuclear and Particle Physics, 34(12):2581, 2007.

[25] J. N. Matthews. A Heitler model of extensive air showers. Astroparticle Physics, 22:387-397, 2005.

[26] J. N. Matthews et al. for the Telescope Array Collaboration. Overview of the Telescope Array Experiment. In Proceedings of the 31st ICRC, Lódź, Poland, number 1386, 2009. http://icrc2009.uni.lodz.pl/proc/html/.

[27] G. A. Askaryan. Excess negative charge of an electron-photon shower and its coherent radio emission. Soviet Physics JETP, 14:441, 1962.

[28] F. D. Kahn and I. Lerche. Radiation from cosmic ray air showers. In Proceedings of the Royal Society of London. Series A, Mathematical and Physical Sciences, volume 289, page 206, 1966. 
[29] J. V. Jelley, J. H. Fruin, N. A. Porter, et al. Radio Pulses from Extensive Cosmic-Ray Air Showers. Nature, 205:327-328, 1965.

[30] H. Falcke et al. - LOPES Collaboration. Detection and imaging of atmospheric radio flashes from cosmic ray air showers. Nature, 435:313-316, 2005.

[31] M. Ender. Radiodetektion von Luftschauern unter dem Einfluss starker elektrischer Felder in der Atmosphäre. FZKA Report 7506, Forschungszentrum Karlsruhe, 2009.

[32] M. Ender et al. - LOPES Collaboration. Radio Emission of Extensive Air Showers during Thunderstorms. In Proceedings of the 31st ICRC, Eódź, Poland, number 0405, 2009. http://icrc2009.uni.lodz.pl/proc/html/.

[33] T. Huege, M. Ludwig, O. Scholten, and K. D. de Vries. The convergence of EAS radio emission models and a detailed comparison of REAS3 and MGMR simulations. Nuclear Instruments and Methods in Physics Research Section A: Accelerators, Spectrometers, Detectors and Associated Equipment, 662(Supplement 1):S179 - S186, 2012. ARENA 2010.

[34] W. D. Apel et al. - LOPES Collaboration. Lateral distribution of the radio signal in extensive air showers measured with LOPES. Astroparticle Physics, 32:294-303, 2010 .

[35] C. W. James, H. Falcke, T. Huege, and M. Ludwig. General description of electromagnetic radiation processes based on instantaneous charge acceleration in "endpoints". Physical Review E, 84:056602, 2011.

[36] M. Ludwig and T. Huege. REAS3: Monte Carlo simulations of radio emission from cosmic ray air showers using an 'end-point' formalism. Astroparticle Physics, 34(6):438 - 446, 2011.

[37] T. Huege and H. Falcke. Radio emission from cosmic ray air showers. Monte Carlo simulations. Astronomy $\mathcal{E}$ Astrophysics, 430:779-798, 2005.

[38] T. Huege and H. Falcke. Radio emission from cosmic ray air showers: Simulation results and parametrization. Astroparticle Physics, 24:116, 2005.

[39] O. Scholten, K. Werner, and F. Rusydi. A macroscopic description of coherent geo-magnetic radiation from cosmic-ray air showers. Astroparticle Physics, 29:94-103, 2008.

[40] K. Werner and O. Scholten. Macroscopic Treatment of Radio Emission from Cosmic Ray Air Showers based on Shower Simulations. Astroparticle Physics, 29:393-411, 2008.

[41] O. Scholten, K. D. de Vries, and K. Werner. Coherent radiation from extensive air showers. Nuclear Instruments and Methods in Physics Research Section A: Accelerators, Spectrometers, Detectors and Associated Equipment, 662(Supplement 1):S80 - S84, 2012. ARENA 2010. 
[42] T. Huege and H. Falcke. Radio emission from cosmic ray air showers. Coherent geosynchrotron radiation. Astronomy \& Astrophysics, 412:19-34, 2003.

[43] G. A. Askaryan. Coherent radio emission from cosmic showers in air and in dense media. Soviet Physics JETP, 21:658, 1965.

[44] S. Buitink et al. - LOPES Collaboration. Amplified radio emission from cosmic ray air showers in thunderstorms. Astronomy \& Astrophysics, 467:385-394, 2007.

[45] P. W. Gorham et al. - WMAP. Observations of microwave continuum emission from air shower plasmas. Physical Review D, 78(3):032007, 2008.

[46] A. Horneffer et al. - LOPES Collaboration. Primary Particle Energy Calibration of the EAS Radio Pulse Height. In Proceedings of the 30th ICRC, Merida, Mexico, volume 4, pages 83-86, 2007.

[47] S. Nehls. Calibrated Measurements of the Radio Emission of Cosmic Ray Air Showers. FZKA Report 7440, Forschungszentrum Karlsruhe, 2008.

[48] D. Ardouin et al. - CODALEMA Collaboration. Radio-detection signature of high-energy cosmic rays by the CODALEMA experiment. Nuclear Instruments and Methods in Physics Research A, 555:148-163, 2005.

[49] Proceedings of the 4th International workshop on Acoustic and Radio EeV Neutrino detection Activities, ARENA 2010, Nantes, France. published in: Nuclear Instruments and Methods in Physics Research A, 2012. http://arena2010.in2p3.fr/.

[50] H. R. Allan. Radio Emission From Extensive Air Showers. Progress in Elementary Particle and Cosmic Ray Physics, Vol. 10:171-302, 1971.

[51] A. Haungs et al. - KASCADE-Grande Collaboration. Latest Results and Perspectives of the KASCADE-Grande EAS Facility. Nuclear Instruments and Methods in Physics Research Section A: Accelerators, Spectrometers, Detectors and Associated Equipment, 662(Supplement 1):S150 - S156, 2012. ARENA 2010 .

[52] T. Huege et al. - LOPES Collaboration. The LOPES experiment - recent results, status and perspectives. Nuclear Instruments and Methods in Physics Research Section A: Accelerators, Spectrometers, Detectors and Associated Equipment, 662(Supplement 1):S72 - S79, 2012. ARENA 2010.

[53] F. G. Schröder et al. - LOPES Collaboration. New measurements of cosmic ray air showers with the digital radio interferometer LOPES. Astrophysics and Space Sciences Transactions, 7(3):303-306, 2011. 22nd ECRS 2010, Turku, Finland.

[54] H. Röttgering et al. LOFAR: a new radio telescope for low frequency radio observations: science and project status. In Texas in Tuscany. XXI Symposium on Relativistic Astrophysics, pages 69-76, 2003. 
[55] H. Falcke et al. - LOFAR. A very brief description of LOFAR - the Low Frequency Array. In IAU GA 2006, Highlights of Astronomy, volume 14, 2006. astro-ph/0610652.

[56] D. Ardouin et al. - CODALEMA Collaboration. Radioelectric field features of extensive air showers observed with CODALEMA. Astroparticle Physics, 26(4-5):341 - 350, 2006.

[57] D. Ardouin et al. - CODALEMA Collaboration. Geomagnetic origin of the radio emission from cosmic ray induced air showers observed by CODALEMA. Astroparticle Physics, 31(3):192 - 200, 2009.

[58] S. Fliescher for the Pierre Auger Observatory. Radio detection of cosmic ray induced air showers at the Pierre Auger Observatory. Nuclear Instruments and Methods in Physics Research Section A: Accelerators, Spectrometers, Detectors and Associated Equipment, 662(Supplement 1):S124 - S129, 2012. ARENA 2010 .

[59] N. M. Budnev et al. - Tunka Collaboration. The Tunka-133 EAS Chrenkov array - status, first results and plans. In Proceedings of the 31st ICRC, Eódź, Poland, number 1069, 2009. http://icrc2009.uni.lodz.pl/proc/html/, astroph/1003.0089.

[60] S. P. Knurenko et al. $32 \mathrm{MHz}$ radio measurements at the Yakutsk EAS array. In 22nd ECRS, Turku, Finland, 2010.

[61] D. Ardouin et al. - TIANSHAN Collaboration. First detection of extensive air showers by the trend self-triggering radio experiment. Astroparticle Physics, 34(9):717 - 731, 2011.

[62] O. Martineau-Huynh, D. Ardouin, C. Cârloganu, et al. First results of the TIANSHAN radio experiment for neutrino detection. Nuclear Instruments and Methods in Physics Research Section A: Accelerators, Spectrometers, Detectors and Associated Equipment, 662(Supplement 1):S29 - S31, 2012. ARENA 2010.

[63] O. Brusova, L. Anchordoqui, T. Huege, and K. Martens. Radio Detection of Neutrinos from Behind a Mountain. In Proceedings of the 30th ICRC, Merida, Mexico, volume 5, pages 1585-1588, 2007.

[64] O. Vedeneev. Depth of the maximum of extensive air showers and mass composition of primary cosmic radiation at an energy of $4 \times 10^{17} \mathrm{eV}$ according to data on radioemission from extensive air showers. Physics of Atomic Nuclei, $72: 250-256,2009$.

[65] J. Auffenberg, D. Besson, T. Gaisser, et al. A radio air shower detector as an extension for IceCube and IceTop. Nuclear Instruments and Methods in Physics Research A, 604(1-2, Supplement 1):S53 - S56, 2009. ARENA 2008.

[66] S.-Y. Sun, P. Chen, and M. Huang. Simulation of the Event Reconstruction of Ultra High Energy Cosmic Neutrinos with Askaryan Radio Array. ArXiv e-prints, 2010. astro-ph/1002.0023. 
[67] I. Kravchenko for the RICE Collaboration. Recent results from the RICE experiment at the South Pole. Nuclear Instruments and Methods in Physics Research Section A: Accelerators, Spectrometers, Detectors and Associated Equipment, 662(Supplement 1):S42 - S45, 2012. ARENA 2010.

[68] H. Landsman, L. Ruckman, and G. S. Varner. AURA-A radio frequency extension to IceCube. Nuclear Instruments and Methods in Physics Research A, 604(1-2, Supplement 1):S70 - S75, 2009. ARENA 2008.

[69] S. Hoover et al. - ANITA Collaboration. Observation of Ultrahigh-Energy Cosmic Rays with the ANITA Balloon-Borne Radio Interferometer. Physical Review Letters, 105(15):151101, 2010.

[70] T. Huege. private communication, 2010.

[71] L. Gerhardt, S. Klein, T. Stezelberger, et al. A prototype station for ARIANNA: A detector for cosmic neutrinos. Nuclear Instruments and Methods in Physics Research A, 624(1):85 - 91, 2010.

[72] A. Connolly. SalSA status. Nuclear Instruments and Methods in Physics Research A, 604(1-2, Supplement 1):S122 - S123, 2009. ARENA 2008.

[73] M. Mevius, S. Buitink, H. Falcke, et al. Detecting ultra high energy neutrinos with LOFAR. Nuclear Instruments and Methods in Physics Research Section A: Accelerators, Spectrometers, Detectors and Associated Equipment, 662(Supplement 1):S26 - S28, 2012. ARENA 2010.

[74] G. A. Gusev, V. A. Chechin, B. N. Lomonosov, et al. Design of the LORD experiment and perspectives of ultrahigh-energy particles observation. $\mathrm{Nu}$ clear Instruments and Methods in Physics Research Section A: Accelerators, Spectrometers, Detectors and Associated Equipment, 662(Supplement 1):S99 S102, 2012. ARENA 2010.

[75] J.A. Aguilar et al. - ANTARES Collaboration. AMADEUS-The acoustic neutrino detection test system of the ANTARES deep-sea neutrino telescope. $\mathrm{Nu}$ clear Instruments and Methods in Physics Research A, 626-627:128 - 143, 2011.

[76] T. Karg. Status and recent results of the South Pole Acoustic Test Setup. Nuclear Instruments and Methods in Physics Research Section A: Accelerators, Spectrometers, Detectors and Associated Equipment, 662(Supplement 1):S36 S41, 2012. ARENA 2010.

[77] S. Nehls, A. Hakenjos, M. J. Arts, et al. Amplitude calibration of a digital radio antenna array for measuring cosmic ray air showers. Nuclear Instruments and Methods in Physics Research A, 589(3):350 - 361, 2008.

[78] T. Antoni et al. - KASCADE Collaboration. The Cosmic-Ray Experiment KASCADE. Nuclear Instruments and Methods in Physics Research A, 513(3):490-510, 2003. 
[79] W. D. Apel et al. - KASCADE-Grande Collaboration. The KASCADE-Grande experiment. Nuclear Instruments and Methods in Physics Research A, 620:202$216,2010$.

[80] F. G. Schröder, T. Asch, L. Bähren, et al. New method for the time calibration of an interferometric radio antenna array. Nuclear Instruments and Methods in Physics Research Section A: Accelerators, Spectrometers, Detectors and Associated Equipment, 615(3):277-284, 2010.

[81] O. Krömer. Empfangssystem zur Radioobservation hochenergetischer kosmischer Schauer und sein Verhalten bei Selbsttriggerung. FZKA Report 7396, Forschungszentrum Karlsruhe, 2008.

[82] T. Asch. Self-Triggering of Radio Signals from Cosmic Ray Air Showers. FZKA Report 7459, Forschungszentrum Karlsruhe, 2009.

[83] A. Horneffer. Measuring Radio Emission from Cosmic Ray Air Showers with a Digital Radio Telescope. PhD Thesis, Rheinische Friedrich-WilhelmsUniversität Bonn, Germany, 2006. http://nbn-resolving.de/urn:nbn:de:hbz:5N07819 .

[84] Information about the Earth's magnetic field. http://www-app3.gfzpotsdam.de/Declinationcalc/declinationcalc.html.

[85] P. G. Isar. Polarization Measurements of Radio Emission of Cosmic Ray Air Showers with LOPES. PhD Thesis, Karlsruhe Institute of Technology, 2010. http://digbib.ubka.uni-karlsruhe.de/volltexte/1000017925.

[86] D. Huber. Messung des dreidimensionalen Polarisationsvektors der Radioemission aus Luftschauern. Diploma Thesis, Karlsruhe Institute of Technology, 2010. http://www.astro.ru.nl/lopes/publications.

[87] H. Nyquist. Certain Topics in Telegraph Transmission Theory. American Institute of Electrical Engineers, Transactions of the, 47(2):617 -644, 1928.

[88] LOFAR software package. http://usg.lofar.org/.

[89] A. Nigl et al. - LOPES Collaboration. Direction identification in radio images of cosmic-ray air showers detected with LOPES and KASCADE. Astronomy E Astrophysics, 487:781-788, 2008.

[90] J. Abraham et al. - Pierre Auger Observatory. Trigger and aperture of the surface detector array of the Pierre Auger Observatory. Nuclear Instruments and Methods in Physics Research A, 613(1):29 - 39, 2010.

[91] J. Abraham et al. - Pierre Auger Observatory. The fluorescence detector of the Pierre Auger Observatory. Nuclear Instruments and Methods in Physics Research A, 620(2-3):227 - 251, 2010.

[92] J. Abraham et al. - Pierre Auger Observatory. Measurement of the energy spectrum of cosmic rays above $10^{18} \mathrm{eV}$ using the Pierre Auger Observatory. Physics Letters B, 685(4-5):239 - 246, 2010. 
[93] J. Abraham et al. - Pierre Auger Observatory. Measurement of the Depth of Maximum of Extensive Air Showers above $10^{18} \mathrm{eV}$. Physical Review Letters, 104(9):091101, 2010.

[94] M. Kleifges for the Pierre Auger Observatory. Extension of the Pierre Auger Observatory using high-elevation Fluorescence telescopes (HEAT). In Proceedings of the 31st ICRC, Eódź, Poland, number 0410, 2009. http://icrc2009.uni.lodz.pl/proc/html/, astro-ph/0906.2354.

[95] M. Platino for the Pierre Auger Observatory. AMIGA - Auger Muons and Infill for the Ground Array of the Pierre Auger Observatory. In Proceedings of the 31st ICRC, Eódz,, Poland, number 0184, 2009. http://icrc2009.uni.lodz.pl/proc/html/, astro-ph/0906.2354.

[96] A. van den Berg for the Pierre Auger Observatory. Radio detection of high-energy cosmic rays at the Pierre Auger Observatory. In Proceedings of the 31st ICRC, Eódź, Poland, number 0232, 2009. http://icrc2009.uni.lodz.pl/proc/html/, astro-ph/0906.2354.

[97] J. Coppens for the Pierre Auger Observatory. Observation of radio signals from air showers at the Pierre Auger Observatory. Nuclear Instruments and Methods in Physics Research Section A: Accelerators, Spectrometers, Detectors and Associated Equipment, 604(1-2, Supplement 1):S41 - S43, 2009. ARENA 2008.

[98] B. Revenu for the Pierre Auger Observatory. Radio-detection of cosmic ray air showers by the RAuger experiment, a fully autonomous and self-triggered system installed at the Pierre Auger Observatory. Nuclear Instruments and Methods in Physics Research Section A: Accelerators, Spectrometers, Detectors and Associated Equipment, 662(Supplement 1):S130 - S133, 2012. ARENA 2010.

[99] O. Krömer et al. - LOPES Collaboration. New Antenna for Radio Detection of UHECR. In Proceedings of the 31st ICRC, Eódz, Poland, number 1232, 2009. http://icrc2009.uni.lodz.pl/proc/html/.

[100] M. Stephan for the Pierre Auger Observatory. Antennas, Filters and Preamplifiers designed for the Radio Detection of Ultra-High-Energy Cosmic Rays. In Proceedings of the Asia-Pacific Microwave Conference, Yokohama, Japan, 2010 .

[101] S. Argirò, S. L. C. Barroso, J. Gonzalez, et al. The offline software framework of the Pierre Auger Observatory. Nuclear Instruments and Methods in Physics Research A, 580(3):1485 - 1496, 2007.

[102] S. Fliescher, D. Fraenkel, B. Fuchs, et al. The radio extension of Auger Offline. GAP note 2010-056, Pierre Auger Observatory, 2010.

[103] P. Abreu et al. - Pierre Auger Observatory. Advanced functionality for radio analysis in the Offline software framework of the Pierre Auger Observatory. 
Nuclear Instruments and Methods in Physics Research A, 635(1):92 - 102, 2011.

[104] I. C. Maris, F. Schüssler, R. Ulrich, and M. Unger. Data Summary Trees and Shower Visualization for Reconstructed Auger Events. GAP note 2006-081, Pierre Auger Observatory, 2006.

[105] H. Falcke and P. W. Gorham. Detecting radio emission from cosmic ray air showers and neutrinos with a digital radio telescope. Astroparticle Physics, 19:477-494, 2003.

[106] T. Huege, R. Ulrich, and R. Engel. Monte Carlo simulations of geosynchrotron radio emission from CORSIKA-simulated air showers. Astroparticle Physics, $27: 392-405,2007$.

[107] S. Lafebre, H. Falcke, J. Hörandel, et al. Prospects for determining air shower characteristics through geosynchrotron emission arrival times. Astroparticle Physics, 34(1):12 - 17, 2010.

[108] E. Hecht. Optics (4th Edition). Addison Wesley, 2001.

[109] M. Ageron et al. - ANTARES Collaboration. The ANTARES optical beacon system. Nuclear Instruments and Methods in Physics Research A, 578(3):498 $-509,2007$.

[110] A. Silvestri et al. - ANITA Collaboration. Status of ANITA and ANITA-lite. In Neutrinos and Explosive Events in the Universe, volume 209 of NATO Science Series, pages 297-306. Springer Netherlands, 2005.

[111] K. Hoffman et al. - AURA Collaboration. AURA: The Askaryan Underice Radio Array. Journal of Physics: Conference Series, 81(1):012022, 2007.

[112] M. Yamamoto. Digital beacon receiver for ionospheric TEC measurement developed with GNU Radio. Earth Planets Space, 60(11):e21-e24, 2008.

[113] C. Timmermans, J. Kelley, and F. G. Schröder. Radio beacon measurements and GPS-Timing. GAP note 2009-071, Pierre Auger Observatory, 2009.

[114] P. Vyskocil and J. Sebesta. Relative timing characteristics of GPS timing modules for time synchronization application. In International Workshop on Satellite and Space Communications, 2009, IWSSC 2009, pages 230 -234, 2009.

[115] F. G. Schröder, H. Bozdog, and O. Krömer. Test Measurements for the Time Calibration of AERA with a Beacon. GAP note 2009-085, Pierre Auger Observatory, 2009.

[116] M. Konzack. Entwicklung eines Beacons zur externen Zeitkalibration für das Auger Engineering Radio Array. Diploma Thesis, Karlsruhe Institute of Technology, 2010. http://www.astro.ru.nl/lopes/publications.

[117] M. Konzack, H. Bozdog, A. Haungs, and F. G. Schröder. Specifications of the AERA Beacon and its Software. GAP note 2010-099, Pierre Auger Observatory, 2010. 
[118] O. Krömer. private communication, 2010.

[119] F. G. Schröder et al. - LOPES Collaboration. On noise treatment in radio measurements of cosmic ray air showers. Nuclear Instruments and Methods in Physics Research Section A: Accelerators, Spectrometers, Detectors and Associated Equipment, 662(Supplement 1):S238 - S241, 2012. ARENA 2010.

[120] ITU (International Telecommunication Union). Radio noise. Recommendation ITU-R P.372-10, ITU, Geneva, Switzerland, 2009.

[121] CCIR (International Radio Consultative Committee). Worldwide minimum external noise levels, $0.1 \mathrm{~Hz}$ to $100 \mathrm{GHz}$. CCIR Report 670, ITU, Geneva, Switzerland, 1990.

[122] K.-D. Kammeyer. Nachrichtenübertragung (4. Ausgabe). B.G. Teubner, Reihe Informationstechnik, Stuttgart, Deutschland, 2008.

[123] M. Ludwig and T. Huege. REAS3: A revised implementation of the geosynchrotron model for radio emission from air showers. Nuclear Instruments and Methods in Physics Research Section A: Accelerators, Spectrometers, Detectors and Associated Equipment, 662(Supplement 1):S164 - S167, 2012. ARENA 2010.

[124] K. D. de Vries, O. Scholten, and K. Werner. Macroscopic geo-magnetic radiation model; polarization effects and finite volume calculations. Nuclear Instruments and Methods in Physics Research Section A: Accelerators, Spectrometers, Detectors and Associated Equipment, 662(Supplement 1):S175 - S178, 2012. ARENA 2010.

[125] A. Lecacheux, A. Bellétoile, and the CODALEMA Collaboration. Radio signature of extensive air showers observed with the Nançay Decameter Array. In Proceedings of the 31st ICRC, Eódź, Poland, number 1508, 2009. http://icrc2009.uni.lodz.pl/proc/html/.

[126] N. Palmieri. under preperation. PhD Thesis, Karlsruhe Institute of Technology, $2012 ?$

[127] F. Cossavella. Measurements of high energy cosmic rays above $10 \mathrm{PeV}$ with KASCADE-Grande. FZKA Report 7513, Forschungszentrum Karlsruhe, 2009.

[128] R. Glasstetter. Cosmic Ray Energy And Mass Estimator. http://wwwik.fzk.de/ ralph/CREAM.php.

[129] M. Wommer. Bestimmung des Energiespektrums und der mittleren Masse kosmischer Strahlung mit dem KASCADE-Grande-Experiment. Diploma thesis.

[130] A. Nigl et al. - LOPES Collaboration. Frequency spectra of cosmic ray air shower radio emission measured with LOPES. Astronomy $\&$ Astrophysics, 488:807-817, 2008.

[131] T. Bergmann, R. Engel, D. Heck, et al. One-dimensional hybrid approach to extensive air shower simulation. Astroparticle Physics, 26(6):420 - 432, 2007. 
[132] S. Ostapchenko. QGSJET-II: results for extensive air showers. Nuclear Physics B - Proceedings Supplements, 151(1):147 - 150, 2006. ISVHECRI 2004.

[133] S. A. Bass, M. Belkacem, M. Bleicher, et al. Microscopic models for ultrarelativistic heavy ion collisions. Progress in Particle and Nuclear Physics, 41:255 $-369,1998$.

[134] K. Link. private communication, 2010.

[135] T. Huege, R. Ulrich, and R. Engel. Dependence of geosynchrotron radio emission on the energy and depth of maximum of cosmic ray showers. Astroparticle Physics, 30:96-104, 2008.

[136] M. Roth. private communication, 2010.

[137] F. G. Schröder et al. - LOPES Collaboration. Investigation of the Radio Wavefront of Air Showers with LOPES and REAS3. In Proceedings of the 32nd ICRC, Beijing, China, volume 3, page \#0313, 2011. www.ihep.ac.cn/english/conference/icrc2011/paper.

[138] M. Ludwig. Modelling of radio emission from cosmic ray air showers . PhD Thesis, Karlsruhe Institute of Technology, 2011. http://digbib.ubka.unikarlsruhe.de/volltexte/1000023592.

[139] ROOT - A Data Analysis Framework. http://root.cern.ch.

[140] T. Antoni et al. - KASCADE Collaboration. Measurements of attenuation and absorption lengths with the KASCADE experiment. Astroparticle Physics, 19(6):703 - 714, 2003.

[141] M. Bertaina et al. - KASCADE-Grande Collaboration. The all particle energy spectrum of KASCADE-Grande in the energy region $10^{16}-10^{18} \mathrm{eV}$ by means of the $N_{\mathrm{ch}}-N_{\mu}$ technique. In Proceedings of the 31st ICRC, Eódz, Poland, number 0323, 2009. http://icrc2009.uni.lodz.pl/proc/html/.

[142] J. Oertlin. Radio Data Analysis of a Prototype Antenna at the Tunka133 Experiment. Bachelor Thesis, Karlsruhe Institute of Technology, 2011. http://tunka-hrjrg.desy.de/e98268/e98271.

[143] S. F. Berezhnev et al. - Tunka Collaboration. The Tunka-133 EAS Cherenkov light array: Status of 2011. In Nucl. Instr. and Meth. A; Proceedings of RICAP 2011, Roma, Italy, 2011. DOI:10.1016/j.nima.2011.12.091, in press.

[144] Tunka-25 EAS Cherenkov Light Array. http://dbserv.sinp.msu.ru/tunka/.

[145] R. N. Bracewell. The Fourier Transform and Its Applications (Second Edition, Revised). McGraw-Hill Book Company, 1986. 



\section{List of Figures}

2.1. Cosmic ray energy spectrum . . . . . . . . . . . . . . . 4

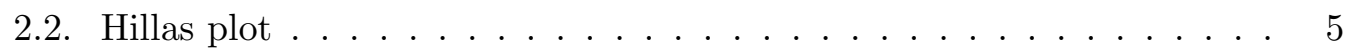

2.3. Air shower scheme . . . . . . . . . . . . . . . . 6

2.4. Simulated radio emission: spectra and pulses . . . . . . . . . . . 10

3.1. Map of LOPES . . . . . . . . . . . . . . . . . . . . 19

3.2. LOPES antennas . . . . . . . . . . . . . . . 20

3.3. LOPES hardware setup . . . . . . . . . . . . . . . 21

3.4. Typical LOPES event . . . . . . . . . . . . . . . . 23

3.5. Map of the Pierre Auger Observatory . . . . . . . . . . . . . . . 25

3.6. Photo of Auger detectors . . . . . . . . . . . . . 2 26

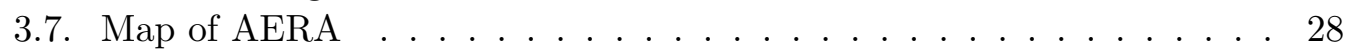

3.8. AERA station . . . . . . . . . . . . . . . . . . . . 29

4.1. 'Perfect' event with high signal-to-noise ratio . . . . . . . . . . 32

4.2. Influence of additional timing uncertainty on CC beam . . . . . . . . . 34

4.3. Setup for delay measurements . . . . . . . . . . . . . . . . 38

4.4. Example for delay measurement . . . . . . . . . . . . . . . . 39

4.5. Deviations between different delay calculation methods . . . . . . . . . 40

4.6. Phase response of LOPES analog electronics . . . . . . . . . . . . . . 42

4.7. Pulse distortion by bandpass filter . . . . . . . . . . . . . . 43

4.8. LOPES beacon antennas . . . . . . . . . . . . . . . 45

4.9. Example for beacon phase differences . . . . . . . . . . . . . 47

5.1. Photos: measurements with the preliminary test beacon at Coihueco . 52

5.2 . Beacon power received in the AERA site . . . . . . . . . . 53

5.3. Spectrum of MAXIMA 4 with beacon signal . . . . . . . . . . . 55

5.4. Relative time deviation between MAXIMA 3 and $4 \ldots \ldots$. . . . . 56

5.5. Improvement of the relative timing with the beacon . . . . . . . 56

5.6. Photo of AERA beacon . . . . . . . . . . . . . . . 59

5.7. Layout of the AERA beacon system . . . . . . . . . . . 6 60

5.8. Scheme of the beacon electronics . . . . . . . . . . . . 60

5.9. Spectrum after the beacon amplifier . . . . . . . . . . 63

5.10. Beacon signals in mean spectrum of AERA station $01 \ldots 63$

5.11. Dynamic spectrum, north-south polarization, AERA station 01 . . . 65

6.1. Radio background at $\mathrm{MHz}$ frequencies . . . . . . . . . . . . 68

6.2. Typical LOPES event with noise and RFI . . . . . . . . . . . . 69

6.3. Example for noise measurement . . . . . . . . . . . . . . . 73

6.4. SNR of pure noise: histogram and dependence on noise window size .74 
6.5. SNR of pure noise: different methods for noise measurement . . . . . . 75

6.6. Test pulses used to study noise influence . . . . . . . . . . . . . . 76

6.7. Input distributions of true signal amplitude . . . . . . . . . . . . 77

6.8. Noise influence on signal amplitude . . . . . . . . . . . . . . 78

6.9. SNRs in individual LOPES antennas . . . . . . . . . . . . . . 79

6.10. Systematic effects in the noise influence on signal amplitudes . . . . 79

6.11. Noise dependent error of amplitude measurements . . . . . . . . . 81

6.12. Relative error and size of noise correction . . . . . . . . . . 81

6.13. Examples for noise influence on lateral distributions . . . . . . . . . 83

6.14. Noise influence on lateral distributions at low and high SNRs . . . . . 83

6.15. Pulse arrival time uncertainty due to noise . . . . . . . . . . . . . 84

6.16. Systematic effects in the pulse arrival time uncertainty . . . . . . . 86

7.1. Example for pulse amplitude measurement . . . . . . . . . . . . 88

7.2. Examples for lateral distributions measured with LOPES . . . . . . . 90

7.3. Correlation of lateral fit parameters . . . . . . . . . . . . . . 91

7.4. Check how well an exponential function fits lateral distributions . . . . 92

7.5. Map of preselected events . . . . . . . . . . . . . . . . . 94

7.6. Quality cut on cross-correlation beam . . . . . . . . . . . 995

7.7. Influence of the bandpass filter on lateral distributions . . . . . . . . . 97

7.8. Influence of up-sampling on lateral distributions . . . . . . . . . . . . . 99

7.9. Examples for REAS3 lateral distribution . . . . . . . . . . . . . . 101

7.10. Amplitude and slope of different REAS versions vs. LOPES data . . . 103

7.11. Lateral distributions flattening towards shower core . . . . . . . . . . . 104

7.12. Lateral slope and amplitude parameter of LOPES and REAS3 . . . . 105

7.13. Per-event deviations between $R_{0}$ of REAS3 and LOPES . . . . . . 105

8.1. Geometrical delay $\tau_{\text {geo }} \ldots \ldots$. . . . . . . . . . . . 110

8.2. Examples for pulse time measurement . . . . . . . . . . . . . . 111

8.3. Examples for pulse arrival time distributions . . . . . . . . . . . 113

8.4. Curvature radius . . . . . . . . . . . . . . . . . . . . 114

8.5. Variable time offset in conical fit . . . . . . . . . . . . . . 115

8.6. Curvatures of cross-correlation beam and arrival time distribution . . 116

8.7. Curvature of LOPES events and REAS3 simulations . . . . . . . . 117

8.8. Curvature vs. shower parameters for LOPES measurements . . . . . . 118

8.9. Curvature vs. shower parameters for REAS3 simulations . . . . . . . 119

8.10. Cone parameter of LOPES events and REAS3 simulations . . . . . . . 120

8.11. Cone parameter vs. shower parameters for LOPES measurements . . . 122

8.12. Cone parameter vs. shower parameters for REAS3 simulations . . . . 123

8.13. Cross-comparison of $\rho$ and $R_{0}$ for LOPES and REAS3 . . . . . . . . 124

8.14. $X_{\max }$ reconstructed with spherical fit . . . . . . . . . . . . 124

8.15. $X_{\max }$ reconstructed with spherical fit: scatter plots . . . . . . . . . . 124

8.16. Correlation between cone parameter $\rho$ and true $X_{\max } \ldots \ldots 127$

8.17. True and reconstructed $X_{\max }$ of REAS3 simulations . . . . . . . 127

8.18. $X_{\max }$ reconstructed with conical fit: scatter plots . . . . . . . . 127

8.19. Zenith vs. $X_{\max }$ for REAS3 simulations $\ldots \ldots \ldots \ldots$

8.20. $X_{\max }$ reconstructed with conical fit: distributions . . . . . . . . . . 129 
8.21. $X_{\max }$ resolution for simulations . . . . . . . . . . . . . . . . 129

8.22. $X_{\max }$ vs. zenith and lateral distance for LOPES events . . . . . . . . 130

8.23. $X_{\max }$ vs. KASCADE-Grande mass estimator . . . . . . . . . . 131

8.24. $X_{\max }$ vs. energy . . . . . . . . . . . . . . . 132

A.1. Map of Tunka and photo of SALLA at Tunka . . . . . . . . . . . . 138

A.2. Tunka background spectrum and example event . . . . . . . . . . . 139

A.3. Radio pulses: coincident pulse, and arrival time distribution . . . . . . 140

A.4. Energy and lateral distance of PMT and radio events . . . . . . . . . . 141

D.1. Event GT $1134526224 \ldots \ldots$. . . . . . . . . . . . . . 148

D.2. Event GT $1143399650 \ldots \ldots$. . . . . . . . . . . . . 148

D.3. Event GT $1147154100 \ldots \ldots$. . . . . . . . . . . . . . 149

D.4. Event GT $1149141637 \ldots \ldots 149$

D.5. Event GT $1153878239 \ldots \ldots 150$

D.6. Event GT $1160717553 \ldots \ldots 150$

D.7. Event GT $1137055868 \ldots \ldots 151$

D.8. Event GT $1145572074 \ldots \ldots$. . . . . . . . . . . . 151

D.9. Event GT $1146534199 \ldots \ldots 152$

D.10.Event GT $1146914254 \ldots \ldots \ldots$

D.11.Event GT $1148046880 \ldots \ldots \ldots \ldots$

D.12.Event GT $1157273315 \ldots \ldots \ldots$

D.13.Event GT $1183540832 \ldots \ldots \ldots \ldots \ldots \ldots$

D.14.Event GT $1212998406 \ldots \ldots$. . . . . . . . . . . . . 154

D.15.Event GT $1220969642 \ldots \ldots \ldots \ldots \ldots$

D.16.Event GT $1233776240 \ldots \ldots \ldots \ldots \ldots$

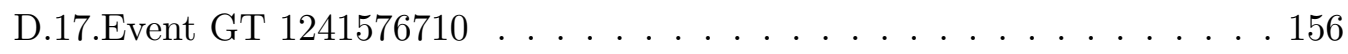

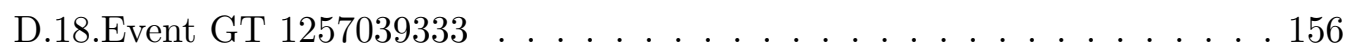

D.19.Event GT $1173208699 \ldots \ldots \ldots \ldots$

D.20.Event GT $1203688126 \ldots \ldots \ldots \ldots$

D.21.Event GT $1216623931 \ldots \ldots \ldots \ldots$

D.22.Event GT $1225582507 \ldots \ldots \ldots \ldots$

D.23.Event GT $1237071988 \ldots \ldots \ldots \ldots$

D.24.Event GT $1252956440 \ldots \ldots \ldots$ 



\section{List of Tables}

2.1. Comparison of air shower detection techniques $\ldots \ldots . \ldots 9$

4.1. LOPES coordinate system . . . . . . . . . . . . . . 36

4.2. Conformance between LOPES and KASCADE coordinate systems . . 37

4.3. Summary of relative timing uncertainties . . . . . . . . . . 50

5.1. Decrease of beacon power with distance: power law indices . . . . . . 54

5.2. Improvement of the relative timing with the beacon . . . . . . . . 57

5.3. Technical specifications of the AERA beacon . . . . . . . . . . 61

5.4. Relative timing precision of AERA . . . . . . . . . . . . . . 65

6.1. Methods tested for noise measurement . . . . . . . . . . . . . 72

6.2. Procedure for testing the consistency criterion . . . . . . . . . . . 72

6.3. SNR of pure noise, for different methods . . . . . . . . . . . . 73

6.4. Different methods for noise measurement relative to default method . 74

7.1. Definition of shower coordinates . . . . . . . . . . . . . 89

7.2. Preselection cuts . . . . . . . . . . . . . . . . 93

7.3. Selection statistics . . . . . . . . . . . . . . . . . 93

7.4. Summary of systematic effects on lateral distributions . . . . . . . . 99

8.1. Selection for pulse arrival time distributions . . . . . . . . . . . 108

C.1. LOPES 30 antenna positions . . . . . . . . . . . . . . 145

C.2. LOPES Dual antenna positions . . . . . . . . . . . . . 146 



\title{
List of Abbreviations
}

\author{
a.s.l. \\ above see level \\ $\mathrm{ADC}$ \\ Analog to Digital Converter \\ AERA \\ Auger Engineering Radio Array \\ AIRES \\ AIRshower Extended Simulations \\ AMIGA \\ Auger Muons and Infill for the Ground Array \\ BLS \\ Balloon Launching Station \\ CC \\ Cross-Correlation \\ cf. \\ confer (compare) \\ CLF \\ Central Laser Facility \\ CODALEMA \\ COsmic ray Detection Array with Logarithmic ElectroMagnetic \\ Antennas \\ CORSIKA COsmic Ray SImulations for KAscade \\ CRS Central Radio Station \\ DAQ Data AcQuisition \\ DC Direct Current \\ e.g. $\quad$ exempli gratia (for example) \\ FD Fluorescence Detector \\ FFT $\quad$ Fast Fourier Transform \\ FM Frequency Modulation \\ FWHM Full Width at Half Maximum \\ GPS Global Positioning System \\ HEAT High Elevation Auger Telescopes \\ i.e. $\quad$ id est (this is) \\ KASCADE KArlsruhe Shower Core and Array DEtector \\ LNA Low Noise Amplifier
}


LOFAR LOw Frequency ARray

LOPES LOFAR PrototypE Station

LOPES ${ }^{\text {STAR }} \quad$ LOPES Self Triggered Array of Radio detectors

LPDA Logarithmic Periodic Dipole Antenna

MAXIMA Multi Antenna eXperiment In Malargüe Argentina

MGMR Macroscopic model of GeoMagnetic Radiation

PMT PhotoMultiplier Tube

REAS Radio Emission from Air Showers

RFI Radio Frequency Interference

RMS Root Mean Square (Following the language use in physics, 'RMS' is used as synonym for 'standard deviation', although the exact mathematical definition is different.)

SALLA Short Aperiodic Loaded Loop Antenna

SD Surface Detector

SNR Signal-to-Noise Ratio

TEC Total Electron Content

TIM Trigger Interface Module 


\section{Acknowledgement}

Many people helped me along the way completing this thesis. I owe a debt of gratitude to all of them, especially to:

Johannes Blümer, my referee, who gave me the opportunity to work in his institute and provided fruitful ideas for this thesis. Without him I would not participate in these exciting cosmic ray experiments.

Hartmut Gemmeke, my co-referee, for his support and expertise in technical questions. The collaboration with his institute was essential for many achievements presented in this thesis.

Andreas Haungs, my boss, who guided me through the whole process of accomplishing this thesis, and helped me to manage any issues - organizational and scientific. I like to thank him in particular for generating a warm working climate which allowed everybody in our group to benefit from each other. He regularly encouraged and motivated me with his inspiring ideas.

Furthermore, I would like to thank my colleagues for their assistance, including proof reading this thesis. I cannot list all of them by name, but I would like to give a special mention to the following people: Tim Huege provided the REAS3 simulations for chapter 7 and contributed to many answers of scientific questions. Steffen Nehls introduced me to LOPES and gave me an excellent example on how to take on responsibility for the experiment. Horia Bozdog taught me that simple solutions are often the better ones, and had excellent ideas on how to improve the precision of our calibration. He contributed essentially to the development of the beacon. Michael Konzack developed the software for controlling the beacon. It was an enriching experience to guide him through his diploma thesis. Marianne Ludwig made a large effort to provide the REAS3 simulations for chapter 8 just in time. Katrin Link and Daniel Huber gave me essential support. Nunzia Palmieri motivated me with her questions. I am glad that I was often among the first to see her interesting results. Oliver Krömer offered solutions to manifold technical issues from his perspective as an engineer. The collaboration with Moses Ender was unfortunately limited to one year. His extraordinary talent was very inspiring. Gina Isar's results often triggered my own studies. Moreover, I like to thank many other colleagues from Karlsruhe, among them my office mates Maximilien Melissas and Benjamin Fuchs, Jan Oertlin, Sabine Bucher, Heike Bolz, Talianna Schmidt, Hans Dembinsiki, Klaus Bekk, Jürgen Wochele, Michael Wommer, Marcel Finger, Adrian Schmidt, Thomas Asch, Christoph Rühle, Marcin Franc, Harald Schieler, Andreas Weindl. 
I am also grateful to the collaborators from other institutes for their support. Therefore, I thank Andreas Horneffer, Lars Bähren, Heino Falcke, Julian Rautenberg, and all colleagues of the LOPES Collaboration, all colleagues of the KASCADEGrande Collaboration, the staff of the Pierre Auger Observatory and all colleagues of the AERA task, and Leonid Kuzmichev and Vasily Prosin of the TUNKA Collaboration.

Special thanks go to those not explicitly mentioned here for proof reading, help and encouragement.

Last but not least, I thank my family and my friends. 